\title{
GRUNDLAGEN DES \\ MAKROSEISMISCHEN \\ ERDBEBENKATALOGS \\ DER SCHWEIZ
}

GABRIELA SCHWARZ-ZANETTI | DONAT FÄH

mit Beiträgen von Virgilio Masciadri und Philipp Kästli

BAND 1

$1000-1680$

(2)

(x) 2

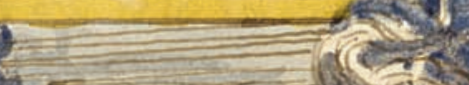

*

ats

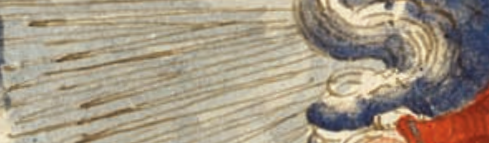

(te 1.

(1)

1.

(1)

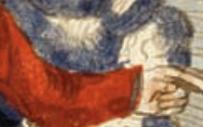

is

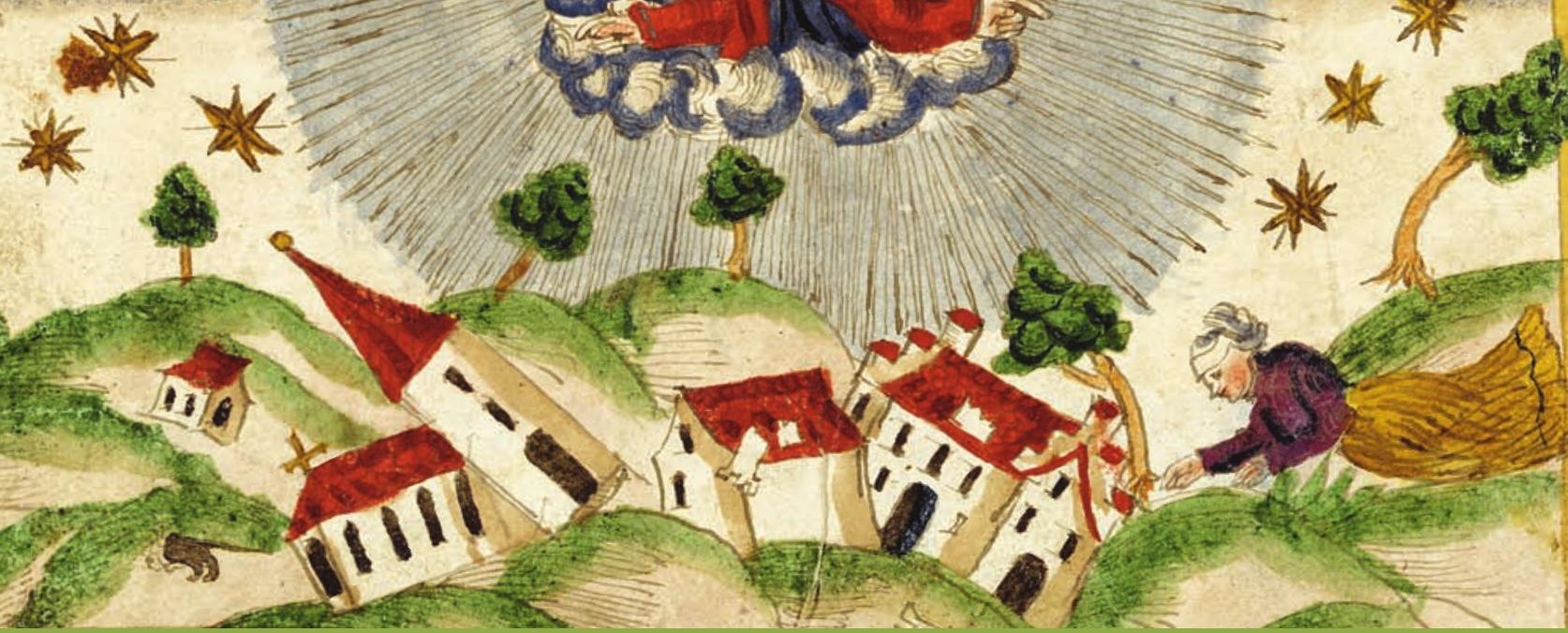

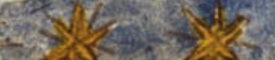

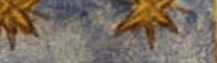

Schweizerischer Erdbebendienst (Hrsg.) 


\section{GRUNDLAGEN DES \\ MAKROSEISMISCHEN ERDBEBEN KATALOGS \\ DER SCHWEIZ}


$A G$ an der

chschulverlag $A G$ an der ETH Zurlutr

zirich volf Hochschulverlag AG an der ETHZZurich

ETHZurich (1) vof Hochschy

der ETHZurich (O) vaf Hochs

Therlag AG an der ETHZZurich (c) vdf HoO

an der ETHZ Zurich (C) vaf HOC

AG an der ETH Zurich (O) vaf HC

ag an der ETHZurich (o vof th

chulverlag AG an der ETH Zurich (C) vd

A $A$ G an der ETH Zurich 


\section{GABRIELA SCHWARZ-ZANETTI | DONAT FÄH}

mit Beiträgen von Virgilio Masciadri und Philipp Kästli

\section{GRUNDLAGEN DES \\ MAKROSEISMISCHEN ERDBEBEN KATALOGS DER SCHWEIZ \\ BAND 1 \\ 1000-1680}

Schweizerischer Erdbebendienst (Hrsg.) 
Publiziert mit Unterstützung des Schweizerischen Nationalfonds zur Förderung der wissenschaftlichen Forschung.

This work is licensed under a creative commons license 通

Coverabbildung:

Zeitgenössische Darstellung des Erdbebens vom 11. März 1584 bei Aigle, VD

(Zentralbibliothek Zürich; Wickiana, Ms F 32f, 19-22)

Bibliografische Information der Deutschen Nationalbibliothek

Die Deutsche Nationalbibliothek verzeichnet diese Publikation in der Deutschen Nationalbibliografie; detaillierte bibliografische Daten sind im Internet über http://dnb.d-nb.de abrufbar.

—ISBN 978-3-7281-3236-9 (Print)

ISBN 978-3-7281-3406-6

DOI 10.3218/3406-6

www.vdf.ethz.ch

顿(c) 2011, vdf Hochschulverlag AG an der ETH Zürich

Umschlaggestaltung: Isabel Thalmann, buchundgrafik.ch 


\section{Inhaltsverzeichnis}

1 Einleitung zum Gesamtkatalog ………………….......................................

1.1 English Summary ...............................................................................

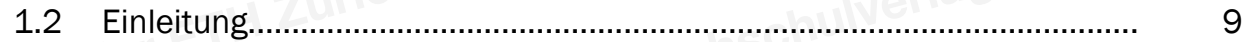

1.3 Dank ........................................................................................ 11

2 Zur Geschichte der Erdbebenbeobachtungen in der Schweiz bis $1680 \ldots \ldots \ldots . . . . \quad 13$

2.1 Einleitung ......................................................................................... 13

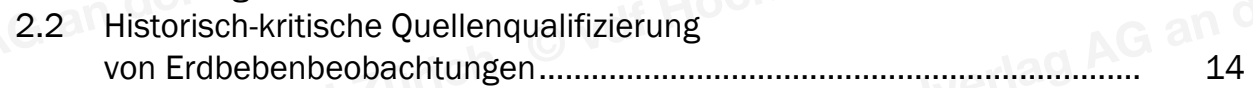

2.3 Quellen und Erdbebenkompilationen ..................................................... 16

2.4 Religiöse Konnotation seismischer Ereignisse ……................................ 17

2.5 Entwicklung des Erdbeben-Beschreibungsmusters im Lauf der Zeit ....... 19

2.6 Das Datenmaterial ............................................................................. 20

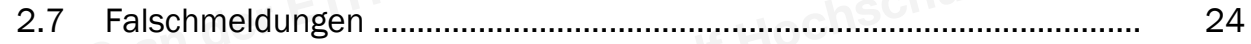

2.8 Neue Funde ................................................................................... 26

2.9 Ausblick ....................................................................................... 26

2.10 Darstellungsschema ........................................................................... 27

2.11 Exkurs: Der Julianische und der Gregorianische Kalender
in der Schweiz

3 Erdbebenkatalog der Schweiz 1000-1680 ……………………………....... 31

4 Abkürzungsverzeichnis ......................................................................... 239

4.1 Kantonskürzel und Länderkürzel......................................................... 239

4.2 Weitere Abkürzungen ........................................................................ 240

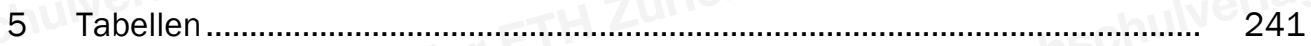

5.1 Tabelle aller Erdbeben der Schweiz mit $l x \geq 6$ von $1000-1680 \ldots \ldots \ldots \ldots . . . \quad 241$

5.2 Tabelle ausgewählter schwacher Erdbeben 1000-1680....................... 242

5.3 Tabelle der Falschmeldungen 1000-1680 ......................................... 244

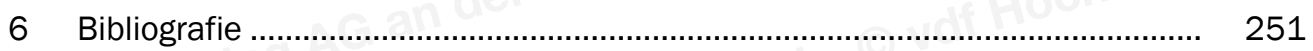

6.1 Manuskripte ......................................................................... 251

6.2 Editionen und Sekundärliteratur....................................................... 254

6.3 Weiterführende Literatur...................................................................... 275

7 Anhang: Die zwölfstufige „Europäische Makroseismische Skala 1998“ (EMS-98) 
Monika Gisler, Donat Fäh

Schweizerischer Erdbebendienst (Hrsg.)

\section{GRUNDLAGEN DES MAKROSEISMISCHEN ERDBEBENKATALOGS DER SCHWEIZ}

\section{BAND 2: 1681-1878}
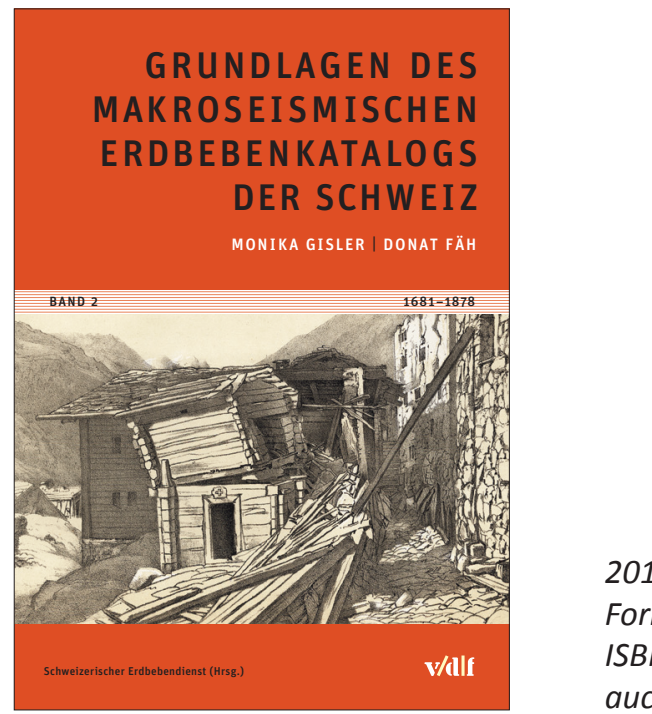

2011, 188 Seiten,

Format $17 \times 24 \mathrm{~cm}$, broschiert

ISBN 978-3-7281-3237-6

auch als E-Book (Open Access) erhältlich

Als Ergänzung zur Online-Version des Schweizer Erdbebenkatalogs ECOS-09 (www. seismo.ethz.ch) steht mit diesen zweibändigen GRUNDLAGEN DES MAKROSEISMISCHEN ERDBEBENKATALOGS DER SCHWEIZ erstmals eine historischkritische Diskussion der wichtigsten nichtinstrumentellen Schweizer Erdbebendaten zur Verfügung. Aus dem Zeitraum 1000-1878 werden alle bekannten Schweizer Erdbeben ab Intensität 6, zahlreiche Falschmeldungen und einige interessante schwache Beben ausführlich kommentiert.

Diese Publikation ist auch als E-Book erhältlich (Open Access).

\section{$\mathbf{v} / \mathbf{l} \mid \mathbf{f}$}

vdf Hochschulverlag AG an der ETH Zürich, VOB D, Voltastrasse 24, CH-8092 Zürich

Tel. +41 (0)44 63242 42, Fax +41 (0)44 63212 32, verlag@vdf.ethz.ch, www.vdf.ethz.ch 


\section{Einleitung zum Gesamtkatalog}

\subsection{English Summary}

The Swiss Seismological Service began practicing historical seismology as an independent field in 1999 in order to establish a consistent national earthquake catalog. A preliminary version of the catalog was launched in the same year (MECOS-99 for the historical part and IECOS-99 for the instrumental part of the catalog). Since then, an interdisciplinary team of historians and seismologists has undertaken a complete revision to compile a new, unified earthquake catalog of Switzerland and its neighboring regions, covering all seismogenic areas that produce a significant contribution to the seismic hazard in Switzerland. The first version of the Earthquake Catalog of Switzerland (ECOS-02) was published in 2002, its replacement will be accessible online in 2011 (www.seismo.ethz.ch).

The core of the historical part of the catalog is MECOS: the Macroseismic Earthquake Catalog of Switzerland. It includes a comprehensive and homogeneous database of historical and macroseismic information, built upon the collection and analysis of historical data. It merges all available macroseismic inputs for significant events within Switzerland: those that reached intensity 6 or higher according to the European Macroseismic Scale (EMS-98) (Grünthal, 1998). MECOS is a key step for upgrading the databases used for earthquake hazard assessment in Switzerland and neighboring regions.

The first few years were dedicated to establishing the catalog by reconstructing intensity fields of known seismic events. In a subsequent step, the catalog was upgraded with selected and generally large earthquakes. Our endeavor concentrated on both quantitative and qualitative analysis through the cooperation of archeologists, seismic engineers, and paleoseismologists.

In studying historical earthquakes, it is attempted to reconstruct them with the aim of surveying the seismic hazard of a particular area. This work involves upgrading existing catalogs or case studies of important earthquakes as well as studying new sources to find more detailed information of both known and, as yet, unknown events. The emphasis was on returning to reliable sources, correcting earthquake parameters, and detecting "fake quakes". False data in previous catalogs had resulted from mixing, extrapolating, or splitting single events, or due to printing or translation errors. The intention was to compile a complete inventory of intensity fields for quakes which were supposed to have damaged localities in Switzerland and neighboring regions, and to derive a unified catalog with uniform source parameters.

A homogeneous quality level for the whole database and catalog has now been achieved, using the European Macroseismic Scale (EMS-98) to establish a consistent macroseis- 
mic field for each event. Macroseismic intensity measures, or rather classifies, the strength of shaking at a particular place, based on observations made of the different effects. An intensity scale provides idealized descriptions of an earthquake's effects, starting with the very weakest (the shaking is imperceivable) to the strongest (everything is totally destroyed). Information on observations during an earthquake is broken down into four groups or sources: human beings, objects, buildings, and the natural environment. These are all scaled by means of numerical values. We assigned a range of intensity by giving both minimum and maximum possible values. This range illuminated information gaps and uncertainties in historical interpretation. This report provides only intensity site points (idps) if analyzed by us, no foreign idps are given.

In what follows we will discuss all known, reliable events that reached the threshold of a maximum intensity of 6 and above. Furthermore, the following includes any supposedly fake or uncertain events that have found their way into any catalog. For each event, the following parameters and information are provided: date, time (in UTC), location (if possible); rating of the event (true event; doubtful event; very doubtful event; fake event due to a time error; fake event due to a location error; fake event); correction of time, date or location (if required); parameters; historical tradition; discussion of the event; and remarks.

All macroseismic data are available on the web (http://www.seismo.ethz.ch/) as part of the Earthquake Catalog of Switzerland. The webpage offers the possibility of searching for earthquakes using different aspects such as magnitude, intensity, date, or region, and provides macroseismic parameters and bibliographical references for each event. The existing catalog spans a period of more than 1,000 years, with the first known event dated AD 849, and a more uncertain one from AD 250 in Augusta Raurica (Augst/CH). Reliable instrumental information has only been available since 1975, so the larger events in the catalog are derived from macroseismic input. All information is stored in a database as well as in the library of the Swiss Seismological Service, ETH Zürich.

MECOS was updated in 2008/2009 and will be presented in two volumes, covering the period from 1000 through to $A D$ 1878. This version (volume 1 ) summarizes our knowledge of events between 1000 and 1680. For the time window 1681 through to 1878 see Gisler and Fäh (volume 2, 2011), roughly covering the enlightenment era and the 19th century ending with the year when the Schweizerische Erdbebenkommission, the Swiss Seismological Commission, was established. From then, the Swiss Seismological Commission produced annual statistics of all earthquakes felt within the Swiss borders; see the annual bulletins of the Swiss Seismological Service (Schweizerische Erdbebenkommission/Schweizerischer Erdbebendienst (1881-1962; 1972-1974)).

This publication should be used as a compendium and has been structured accordingly. An extended discussion of the large earthquakes in Switzerland can be found in Gisler et al. (2008) and in specific scientific publications. 


\subsection{Einleitung}

Am Schweizerischen Erdbebendienst wurde 1999 mit einem interdisziplinären Team von Historikerinnen und Seismologen die Historische Seismologie als Subdisziplin mit dem Ziel etabliert, eine historisch-kritische Revision des historischen Teils des bestehenden nationalen Erdbebenkatalogs durchzuführen (historischer Teil: MECOS-99; instrumenteller Teil: IECOS-99). Eine erste Version des überarbeiteten gesamten Katalogs wurde bereits 2002 online publiziert (ECOS-02), seither wurde sie weiter überarbeitet und 2009 letztmals aufdatiert. Ab 2011 wird sie als ECOS-09 online zugänglich sein (http://www.seismo.ethz.ch/). Ziel der Revision des historischen Teils war einerseits, die Zuverlässigkeit der dokumentarischen Datenbasis für die bisherigen Gefährdungsanalysen historisch-kritisch zu überprüfen. Andererseits sollten Neufunde von Berichten die Datenbasis verbreitern. Zahlreiche historische Irrtümer wie Kalenderfehler, Fehllokalisierungen und Verwechslungen konnten so identifiziert werden, was erlaubte, die seismische Gefährdung der einzelnen Regionen zuverlässiger zu bestimmen.

Der Erdbebenkatalog umfasst eine Zeitperiode von rund 1700 Jahren, beginnend mit einem ungesicherten Ereignis um das Jahr 250 in Augusta Raurica (Augst, AG). Vom Frühmittelalter sind nur wenige Berichte überliefert, im Hoch- und Spätmittelalter verdichten sich dann die Meldungen und nehmen in der Neuzeit laufend zu. Verlässliche instrumentelle Daten sind erst ab 1975 vorhanden. Die grossen Ereignisse im Katalog liegen somit im historischen Teil. Alle zugrunde liegenden Daten sind sowohl in einer Datenbank als auch im Archiv des Schweizerischen Erdbebendienstes der ETH Zürich greifbar. Eine Webseite (http://www.seismo.ethz.ch/) erlaubt, unter bestimmten Aspekten wie Zeitfenster, Magnitude, Intensität, Datum und Region nach Erdbeben zu suchen. Aufgeführt sind sowohl die Parameter eines Ereignisses als auch Angaben zur Makroseismik und zur Bibliografie.

Der Makroseismische Katalog (MECOS) des Erdbebenkatalogs der Schweiz (ECOS) umfasst eine umfangreiche homogene Datenbank von historischen und makroseismischen Informationen und deren Analyse. Makroseismische Daten für alle bekannten bedeutenden Erdbeben der Schweiz, also zumindest alle ab Intensität 6 gemäss EMS-98, sind zugänglich. Abgebildet werden alle bearbeiteten Parameter.

Ausgangspunkt der Revision waren die Aussagen älterer Erdbebenkompilationen, wie zum Beispiel die Arbeit von Volger (1857) oder der Makroseismische Erdbebenkatalog von 1999, MECOS-99. Zentral aber war der nachfolgende Schritt, die qualitative und quantitative Verbesserung der Datenbasis durch eine systematische Suche nach zeitgenössischen Berichten bekannter und noch unbekannter Beben in Archiven und Bibliotheken. Danach galt es, die historische Zuverlässigkeit aller Berichte zu einem Einzelbeben gemäss einem vierteiligen Schema zu bewerten. Als „sicher“ wurden Ereignisse mit zwei bis drei und mehr zeitgenössischen Berichten eingestuft, als „unsicher“ mit ein bis zwei Berichten und als „sehr unsicher“ mit einem Einzelbericht, sei es eine detaillierte Abschrift einer unbekannten Quelle oder manchmal ein allzu knappes Original. Der Kategorie „falsch“ wurden fehlerhafte Berichte oder Aussagen aus nicht überprüfbaren 
Kurzkompilationen zugeteilt. Damit sollte ein möglichst umfassendes und qualifiziertes Datenset für die Ableitung der Parameter des Erdbebenkatalogs zur Verfügung gestellt werden. Für zahlreiche Ereignisse ergaben sich kleinere bis fundamentale Korrekturen an der Datierung, Lokalisierung oder an der Intensität. Mit Befunden aus der Archäologie, dem Ingenieurwesen sowie der Paläoseismologie wurde die interdisziplinäre Datenbasis weiter verstärkt.

Ein konformer Qualitätsstandard für die gesamte Datenbank und den Katalog konnte nun erreicht werden. Zur makroseismischen Auswertung wurde die einheitliche Skala EMS-98 (Grünthal, 1998) angewendet, um ein möglichst homogenes makroseismisches Feld für jedes Ereignis zu erhalten. Die makroseismische Intensität klassifiziert die Stärke der Erschütterung an einem bestimmten Ort, basierend auf der Beobachtung während eines Erdbebens (vgl. Anhang). Die Intensitätsskala bietet eine idealisierte Beschreibung von Erdbebeneffekten an, beginnend mit dem kleinsten Ausmass (die Erschütterung ist nicht wahrnehmbar) bis hin zum stärksten (komplette Zerstörung). Informationen werden in vier Beobachtungsgruppen eingeteilt: Mensch, Objekte, Gebäude und Umwelt. Die Ergebnisse der Beobachtungen an der jeweiligen Gruppe durch einzelne oder mehrere Personen werden mittels numerischer Werte skaliert. Ein Intensitätsbereich wurde bestimmt, indem zunächst eine Minimal- und eine Maximalintensität ( $\left.I_{\min }, I_{\max }\right)$ pro Ort ermittelt wurde, um dann die wahrscheinlichste Intensität $\left(I_{w}\right)$ festzulegen. Diese Spannbreite sollte die Unsicherheit, die bei einer solchen Bestimmung naturgemäss auftritt, ausdrücken.

Mit der Publikation des vorliegenden Doppelbandes wird der momentane Forschungsstand zahlreicher historischer Ereignisse mit weiterführenden Kommentaren und Hinweisen vertieft zugänglich gemacht, d.h., es werden alle Schadensereignisse und alle identifizierten Falschmeldungen, die in früheren Erdbebenkatalogen der Schweiz und des Auslands aufgeführt wurden, sowie einige ausgewählte Schwachbeben vorgestellt.

Der erste Teilband umfasst die Zeit von 1000-1680 und der zweite diejenige von 1681-1878. Letzterer beginnt also mit der Frühaufklärung und endet mit der Gründung der Schweizerischen Erdbebenkommission, der Vorgängerin des Schweizerischen Erdbebendienstes.

Die vorliegende Publikation ist als Handbuch gedacht und entsprechend aufgebaut. Auf ausführlichere Darstellungen zu einzelnen Schweizer Schadenbeben wird laufend verwiesen. Zusammenstellungen sind in „Nachbeben. Eine Geschichte der Erdbeben in der Schweiz“ (Gisler et al., 2008) zu finden. 


\subsection{Dank}

Für die Revision des Schweizerischen Erdbebenkatalogs haben wir von verschiedener Seite Unterstützung erhalten: Schweizerischer Erdbebendienst, ETH Zürich, Schweizerischer Nationalfonds, NAGRA und Swiss Nuclear, Cogito Foundation, Schweizerisches Bundesamt für Zivilschutz, Sicherheitsdepartement Basel Stadt, Jubiläumsstiftung der Schweizerischen Mobiliar Versicherungsgesellschaft, SCOR Schweiz, Aon Re Schweiz, Jubiläumsstiftung der Zürich Versicherungsgruppe. Wir danken allen Beteiligten sehr herzlich für ihren Beitrag. Diese Publikation ist auch Teil des europäischen Projekts NERIES („Network of Research Infrastructures for European Seismology“, Teilprojekt NA4: Distributed Archive of Historical Earthquake Data) - zu erwähnen sind vor allem die konstruktiven Kontakte zu INGV, SisFrance und GFZ. Zu danken ist - neben Domenico Giardini vom Schweizerischen Erdbebendienst - auch zahlreichen Personen in Bibliotheken, Archiven und anderen Institutionen im In- und Ausland, die freundlicherweise Hinweise auf seismische Informationen gegeben haben. Hier sind vor allem Gregor Zenhäusern und seine Mitarbeiter Anne-Lore Bregy und René Pfammatter zu nennen, die aus ihrem seit 2006 laufenden Nationalfonds-Projekt „Klimageschichte des Wallis 1500-1864“ (Brig) Daten zur Verfügung gestellt haben, aber auch Jean Vogt (Strassburg), Pierre Alexandre (Brüssel), Thomas Steinmetz (Wiesbaden), Jürg Muraro (Winterthur), Christian Pfister (Bern), Sylvain Gache (Annecy, F), Jürgen Wolf (Berlin), Sandra Wolff-Ernst (Konstanz), Niculò Gaudenz (Schuls) und andere; für die Korrektur der Tabellen durch Gabriela Gassner-Stamm (SED) und der italienischen Texte durch Nicola Ferrari (Wetzikon) sowie für die umsichtige Betreuung des Druckes durch Angelika Rodlauer vom Hochschulverlag vdf danken wir herzlich. 


\section{Zur Geschichte der Erdbebenbeobachtungen in der Schweiz bis 1680}

Der Historiker ist ein rückwärtsgewandter Prophet.

(F. von Schlegel, 1772-1829)

\subsection{Einleitung}

Das sonst nicht unumstrittene Schlegel-Zitat umschreibt den Zweck der Historischen Seismologie ziemlich zutreffend, denn Erdbeben wiederholen sich in einer bestimmten Region in vergleichbaren Zeiträumen mit ähnlichen Intensitäten; damit leistet ein historisch-krititscher Erdbebenkatalog einen wichtigen Beitrag zur Bestimmung der Erdbebengefährdung.

Der vorliegende erste Band der „Grundlagen des Schweizerischen Erdbebenkatalogs“ stellt den Untersuchungsstand 2009 von wichtigen Schweizer und schweiznahen Erdbeben der Jahre 1000-1680 in standardisierter Form dar. Vorangestellt ist ausserdem ein Hinweis auf Publikationen über die wenigen Informationen zu Ereignissen des ersten nachchristlichen Jahrtausends. Zweck dieser gesamten Zusammenstellung ist es, das Datenmaterial in den wichtigsten Punkten zu präzisieren und für die künftige Suche nach weiteren Quellen und deren Auswertung ein Arbeitsinstrument zur Verfügung zu stellen. Diese Aufgabe erforderte ein interdisziplinäres Team, berührt sie doch nicht nur die Seismologie, sondern basiert auf historisch-kritischer Quellenarbeit, braucht philologische und paläografische Spezialkenntnisse und fallweise auch den Beizug archäologischer Befunde.

Das Hauptziel war der Aufbau einer Datenbank, die auf möglichst zahlreichen zeitgenössischen und einander bestätigenden Beobachtungen basiert, beziehungsweise die Identifizierung ungesicherter oder gar falscher Daten. Zu diesem Zweck wurden sowohl bereits edierte Schriftquellen als auch zahlreiche handschriftliche Dokumente in Archiven und Bibliotheken der Schweiz und des nahen Auslands gesichtet. Zu zuverlässigen Aussagen über den Wert von solchen Dokumenten kann man mit der Methode der historischkritischen Quellenqualifizierung gelangen. Dennoch macht die knappe Datenlage bei zahlreichen Erdbeben des Mittelalters und teilweise noch der Frühen Neuzeit besondere interpretatorische Vorsicht nötig. 


\subsection{Historisch-kritische Quellenqualifizierung von Erdbebenbeobachtungen}

\subsubsection{Unkritische Erdbeben-Kompilationen als bisherige Datenbasis}

Basis bisheriger Erdbebenkataloge für die Schweiz waren meist sogenannte Erdbebenkompilationen, d.h. unkritische Sammlungen schriftlicher Informationen jeglicher Provenienz über vergangene Erdbeben aus verschiedenen Zeiträumen und Gebieten. Da diese aber meist zahlreiche Irrtümer und nur zum Teil Quellenverweise enthalten, waren sie bei der vorliegenden Arbeit nur als Ausgangspunkt für eine unsystematische Suche nutzbar.

Die älteste bekannte Kompilation des Untersuchungsgebiets stammt vom Strassburger Kaplan Fritsche Closener, der sie spätestens 1362 niederschrieb, mit grosser Wahrscheinlichkeit angeregt durch das Villach/Friaul-Beben von 1348 und das Basler Beben von 1356; es handelt sich um eine kurze Liste von Erdbeben der Jahre 1279 bis 1362, die er in seine Chronik einfügte. Ähnlich verhält es sich mit der Arbeit des Theologen und Naturwissenschafters Elie Bertrand von 1756, damals Pfarrer in Bern, die auf das Briger Schadenbeben vom Dezember 1755 folgte, oder mit derjenigen des Geologen Otto Volger aus dem Jahre 1857, die nach dem Schadenbeben von Törbel, VS, im Juli 1855 entstand. Weitere Kompilationen sind in der Bibliografie aufgeführt.

Aus der Unzuverlässigkeit der Kompilationen, die der belgische Historiker Pierre Alexandre für die Jahre 400-1260 in Mitteleuropa auf 40-78\% beziffert, ${ }^{1}$ folgt, dass zur Vermeidung von Fehleinschätzungen Nachrichten über Erdbeben zwingend entweder auf zeitgenössische Originalquellen zurückgeführt oder entsprechend in ihrer Zuverlässigkeit zurückgestuft werden müssen. Kaum benutzbar sind deshalb historische Erdbebenkataloge ohne Zitate und Quellenangaben, wie diejenigen von Lindiner (1711), Journal de Genève (1855), Kopp (1861), Tribolet (1876), Van Gils \& Leydecker (1991) oder Rüttener (1995).

\subsubsection{Historisch-kritisch qualifizierte Erdbebenberichte als neue Datenbasis}

Grundlage für eine zuverlässige Auswertung können also nur Originalquellen sein. Unter einer Originalquelle wird hier eine zeitgenössische Niederschrift verstanden, welche die Beobachtung eines Ereignisses zu einem bestimmten Zeitpunkt an einem identifizierbaren Ort festhält. Typische Beispiele für solche Quellen sind Annalen, Chroniken, Pfarreibücher, Verwaltungsschriften, Tagebücher (besonders Wettertagebücher), Akten, Briefe und Ähnliches. Schwer zugängliche Quellen und neue Funde sind im folgenden Katalogteil oft direkt zitiert. Als historisch gesichert gilt ein Ereignis dann, wenn mindestens zwei bis drei voneinander unabhängige zeitgenössische Beobachtungen vorliegen, die sich gegenseitig bestätigen. Um danach zuverlässig auf die Intensität und das Epizentrum schliessen

1 Alexandre (1990, S. 6). 
zu können, braucht es aber Beobachtungen von verschiedenen Orten; fehlen solche, kann keine Lokalisierung vorgenommen werden. Archäologische Befunde bieten unter Umständen wertvolle Ergänzungen, wie zum Beispiel beim Basler Erdbeben von 1356.

Die im Folgenden vereinfacht dargestellten sprachlichen und historischen Untersuchungsschritte bilden die Grundlage für eine historisch-kritische Bewertung der Zuverlässigkeit jeder einzelnen Quelle.

Transkription: Bei Quellen, für die noch keine Edition nach modernen wissenschaftlichen Standards vorliegt, werden Transkription des Manuskripts und eine Arbeitsedition für die makroseismische Auswertung erstellt.

Übersetzung: $\quad$ Übersetzen v. a. der lateinischen edierten und noch unedierten Texte; grundsätzlich wurden alle Quellen neu aus der Originalsprache übersetzt.

Autor: Identifikation des Verfassers und seiner beruflichen Stellung.

Datierung: Identifikation des Kalenderstils und der regionalen Heiligentage.

Uhrzeit: Historische Uhrzeiten sind kaum überprüfbare Angaben, trotzdem wurden teilweise Umrechnungen von Stundenangaben in Weltzeit (UTC) vorgenommen; Umschreibungen wie „nachmittags“ blieben bestehen.

Niederschrift: Bestimmung der zeitlichen Distanz zwischen Ereignis und Niederschrift bildet ein wichtiges Kriterium für die Beurteilung der Zuverlässigkeit eines Berichts.

Stil: $\quad$ Bestimmung des zeittypischen Beschreibungsstils und Vokabulars.

Lokalisierung: Ort der Abfassung, Beobachtungsort und Fremdinformationen führen zu einem Lokalisierungsvorschlag.

Inhalt: Reaktionen der Beobachter und Schadensbeschreibungen erlauben Rückschlüsse auf die Intensität und werden gemäss der EMS-98 (Grünthal, 1998) bewertet. Die Bestimmung eines zeittypischen Beschreibungsmusters, religiös-kultureller Aspekte und von weiteren Intentionen eines Quellentextes (z. B. didaktische Absichten) erlauben eine genauere Bewertung seiner Zuverlässigkeit.

Fehler: $\quad$ Von grosser Wichtigkeit ist auch die Kennzeichnung ungesicherter oder gar falscher Informationen, die in vielen unkritischen Kompilationen bisher unerkannt kursierten.

Filiation: Die Filiation der früh- und hochmittelalterlichen Annalen konnte hier aus zeitlichen Gründen nicht detailliert erarbeitet werden; vor allem sie könnte weitere Abhängigkeiten zwischen den einzelnen Quellen offen legen. Diese ist insofern zentral, als gerade in dieser Periode ein einmal schriftlich fixierter Satz historischer Daten oft auf dem Weg von Abschrift und Kopie in Annalen und Chroniken verschiedener Klöster und Städte Aufnahme findet und dadurch unter Umständen fälschlich den Eindruck authentischer Beobachtungen an einem bestimmten Ort erweckt.

Diese historisch-kritische Bewertung war der erste Schritt. Als „falsch“ werden demnach eindeutig nachweisbare Fehler und einige nur durch unzuverlässig scheinende kompilatorische Arbeiten überlieferte Berichte aus zweiter Hand (Kopien) bezeichnet. Die Qualifikationen „sehr unsicher“ und „unsicher“ werden Ereignissen zugeteilt, für die nur eine 
einzelne Originalquelle vorliegt, oder dann eine oder mehrere Kopien in Kompilationen mit Quellenverweisen. Entsprechend wird ein Ereignis an sich als „sicher" qualifiziert, sobald es von zwei bis drei oder mehr Originalquellen bezeugt wird.

Erst in einem zweiten Schritt wurde die lokale Aussagekraft bestimmt, entsprechend der Anzahl Informationen pro Ort mit „sehr unsicher“ bis „sicher“. Beben, zu denen bloss Beschreibungen ohne Schäden vorlagen, wurden bei der makroseismischen Intensitätsbestimmung mit den Intensitäten 3 bis 5 bewertet, auch wenn die Quellen sie mit Ausdrücken wie „magnus“ oder „ingens“ („gross“ oder „ausserordentlich“) bezeichnen, denn diese beschreiben eher eine religiös-mentale Komponente des Erlebnisses, als dass sie quantifizierende Angaben bieten (vgl. z. B. das Ereignis vom Dezember 1092, wo ein angeblich grosses Erdbeben die Schläfer nicht geweckt hat). Damit soll die Gefahr einer überhöhten Einschätzung minimiert werden. Denn nur Beben, zu denen ausdrückliche Panik- und Schadensbeschreibungen überliefert sind, wurden Intensitätswerte von 6 und mehr zugeordnet.

Resultat der Revision des Katalogteils 1000-1680 ist nicht nur die definierte Zuverlässigkeit der Aussagen zu jedem einzelnen Beben, sondern mit der Identifizierung von zahlreichen Fehlern auch eine Verkleinerung der bisher angenommenen Häufigkeit historisch fassbarer Beben.

\subsection{Quellen und Erdbebenkompilationen}

Ein Ausgangspunkt für die Suche nach Erdbeben-Schriftquellen waren, wie bereits erwähnt, die bekannten, oft aber unkritischen Kompilationen, zum Beispiel von Johann Jakob Scheuchzer (1706 und 1716), Elie Bertrand (1756), Otto Volger (1857), Rudolf Langenbeck (1892), Mario Baratta (1901), Jacob Candreia (1905), Hans Giessberger (1922), August Sieberg (1940), Frédéric Montandon (1942-43) und Van Gils \& Leydecker (1991); aber auch Online-Kataloge wie der französische Erdbebenkatalog SisFrance, der italienische Katalog DOM4.1 und der historische Erdbebenkatalog der Schweiz aus dem Jahre 1999 (MECOS-99) wurden benutzt; sie sind im Anhang aufgeführt. Für das Früh- und Hochmittelalter konnten zahlreiche zeitgenössische Hinweise dem Erdbebenkatalog von Pierre Alexandre (1990) entnommen werden, der die gedruckten Quellen aus Mitteleuropa und Norditalien für die Jahre 394-1259 systematisch durchsucht, bewertet und so, abgesehen von Weiss (1914), als erster eine historisch-kritische Erdbebenkompilation publiziert hat. Darin sind nicht nur die zeitgenössisch belegbaren Erdbeben in Mitteleuropa aufgelistet, sondern in einem "Catalogue des faux séismes“ auch die als Nachricht kursierenden, aber nicht (mehr) beweisbaren Ereignisse. Dank dieser verdienstvollen Arbeit darf angenommen werden, dass heute der grösste Teil der in bereits edierten Texten enthaltenen zuverlässigen Erdbebeninformationen aus diesem Zeitraum und Gebiet bekannt sein dürfte. Für die Zeit nach 1259 wurden für diesen Katalog viele edierte und handschriftliche Originalquellen neu erschlossen. 


\subsection{Religiöse Konnotation seismischer Ereignisse}

Obwohl die seismischen Informationen im Mittelalter für eine Auswertung äusserst knapp sind, kommen sie im Verhältnis zu anderen Nachrichten in den früh- und hochmittelalterlichen Annalen auffallend häufig vor. Dies ist vor allem das Resultat antiker und christlicher Vorstellungen, die Erdbeben als göttliches Wunderzeichen - oder Prodigium - interpretierten.

Bereits für die Antike sind zahlreiche Hinweise auf Naturphänomene und Katastrophen belegbar, daraus aber zuverlässige Aussagen zu extrahieren, gestaltet sich äusserst schwierig; historisch gesichert sind zum Beispiel das Erdbeben und die Flutwelle von $373 / 2$ v. Chr., die die griechische Stadt Helike verschlangen. ${ }^{2}$ Vielmehr war versucht worden, den ständigen Bedrohungen durch die Natur einen tieferen Sinn zu geben, indem man sie in das komplexe Kommunikationssystem zwischen Menschen und Göttern einordnete. ${ }^{3}$ Naturkatastrophen galten allgemein als Botschaft der Götter, ob als Vorzeichen für künftige Ereignisse oder als Zeichen des Zorns über die auf Erden herrschenden Zustände. ${ }^{4}$ „Erdbeben etwa [...] wurden im griechischen Raum als Werk des Gottes Poseidon angesehen, der schon im homerischen Epos [den] Beinamen ,Erderschütterer trägt.“ ${ }^{5}$ Um das Verhältnis zwischen Göttern und Menschen zu befrieden, wurden unter anderem auch Bittprozessionen für eine kollektive Katastrophenbewältigung unternommen. Gleichzeitig sind schon bei den Vorsokratikern erste naturwissenschaftliche Interpretationsversuche von Erdbeben nachweisbar. Diese Theorien blieben aber auf einen kleinen Kreis Gelehrter beschränkt. ${ }^{6}$

In biblischen Texten werden Erdbeben unterschiedlich gewertet. Ein Beispiel für eine Interpretation als Zorn Gottes zeigt Hiob 9,5-9,6; bei christlichen Autoren des 5. Jahrhunderts lässt sich ein synonymer Gebrauch der Worte Erdbeben (seismos) und Gotteszorn (theomenia) belegen. ${ }^{7}$ Gott kann mit einem Beben aber auch nur seine Anwesenheit zeigen (Moses, Exodus 19,18). Mehrere Erdbeben begleiten auch den Kreuzigungstod Christi (Mt 27, 51-54). Vor allem aber werden Erdbeben als Ankündigung des Weltendes verstanden (Offb. 6,12-16, Lukas 21,11). Zahlreiche Spuren dieses antikchristlichen Verständnisses finden sich bis in die Frühe Neuzeit8, erst danach verbreiten sich zunehmend naturwissenschaftliche Ansätze. Diese religiöse Interpretation seismischer Vorgänge erklärt aber auch, warum das Schwergewicht der älteren ErdbebenBeschreibungen weniger auf dem faktischen Ablauf liegt, als auf dem Versuch, den Zeichencharakter zu interpretieren, und damit auf der didaktischen Funktion als Warnung

2 Masciadri (2008), v.a. S. 117-128: Erdtaucher.

3 Meier (2003), S. 46.

4 Vgl. für die Neuzeit auch Gisler (2007, S. 181f.).

5 Meier (2003), S. 46.

6 Nach Meier (2003), S. 46-54.

7 Maier (2003), S. 47.

8 Zehnder (1976), Kapitel E., Vorzeichen und Orakel, S. 498-518. Darunter werden Kometen, Finsternisse, Himmelserscheinungen, Blitz und Donner, Erdbeben und Ähnliches subsumiert. Zehnder zitiert auch den Chronisten Stumpf (1548), der Erdbeben ausdrücklich als Strafzeichen Gottes aufführt (S. 498). 
vor künftigen selbstverschuldeten Ereignissen. Noch die Erdbeben und der Bergsturz vom März 1584 bei Aigle, VD, wurden als „göttliches Ohrzupfen“, 9 auf das man sogar in Luzern mit einem „Generalcrüzgang“ im April reagierte ${ }^{10}$, und das Erdbeben vom 21. September 1650 in Basel als „Zornzeichen Gottes“11 verstanden. Umgekehrt fehlen Belege für eine Interpretation der Erdbeben als diabolisches Werk. Dies scheint meteorologischen Katastrophen vorbehalten gewesen zu sein, wie Akten von Hexenprozessen zeigen. Erst in Goethes Faust II (1832, Vers 10075 ff) findet sich als Antwort auf Theorien von Vulkanisten eine eher satirische Darstellung von Erdbeben als Resultat von Teufelswerk ${ }^{12}$.

Parallel zu den vor allem biblisch beeinflussten Kommentaren in mittelalterlicheren zeitgenössischen Berichten lassen sich jedoch auch wissenschaftliche Theorien nachweisen. „Albertus Magnus (1193-1280) übernahm Theorien von Anaxagoras und Aristoteles und berücksichtigte dabei auch die arabischen und jüdischen Kommentare." 13 Albertus Magnus sah die Ursachen der Erdbeben im Verstopfen der Poren der Erdkruste durch die Gezeiten des Meeres und durch Regen, aber auch in der felsigen Erdoberfläche, Dämpfen und Winden. Sein Schüler Thomas von Cantimpré verfasste 1241 das „Liber de natura rerum“, worauf sich zu einem grossen Teil auch Konrad von Megenberg mit seinem deutschsprachigen „Buch der Natur“14 aus den Jahren 1348-1350 stützte. Darin ist ein Kapitel den Erdbeben gewidmet; also kurz nach dem grossen Villach/FriaulBeben von 1348 verfasst. Davon sind zahlreiche Abschriften und Drucke bis in die Mitte des 16. Jahrhunderts nachweisbar. Trotz dieser bemerkenswerten Verbreitung finden sich in zeitgenössischen Erdbebenberichten des Mittelalters und der Frühen Neuzeit in der untersuchten Region kaum Hinweise auf wissenschaftliche Überlegungen.

Neben den religiösen Elementen fallen einige toposartige Besonderheiten der Erdbebenbeschreibungen auf; bereits für die Antike spricht Sonnabend (1999) sogar von „Katastrophen-Topoi“. „Wie wenn Monster in den Abgründen brüllten“, beschreibt beispielsweise eine St. Galler Notiz das Erdbeben von 1021 und erinnert mit diesem Vokabular an Ovids Metamorphosen. ${ }^{15}$ Ähnlich verschlingen beim Erdbeben von 1155 angeblich Abgründe eine Burg in der Nähe von Cluny. Dieses Erdbeben-Datum wird zwar mehrfach von anderen Quellen bestätigt, nicht aber die zerstörerische Intensität. Als besonders erschreckend wurde mehrmals Glockengeläut während eines Erdbebens bewertet, als ob es von „unsichtbarer Hand“ in Bewegung gesetzt worden sei. In Cassius Dios Beschreibung des Bebens von $115 \mathrm{n}$. Chr. in Antiochia wiederum überlebt ein unschuldiger

9 Schwarz-Zanetti (2008e, S. 60).

10 Zehnder (1976), S. 206.

11 Zwinger (1650).

12 Faust II, Vers 10081 ff, Mephistopheles: „Die Teufel fingen sämtlich an zu husten, / (...) / Die Hölle schwoll von Schwefelstank und -säure: / Das gab ein Gas! Das ging ins Ungeheure, / So dass gar bald der Länder flache Kruste, / So dick sie war, zerkrachend bersten musste.“ (Goethe, 1950 / 1977, Seite 462 und 812)

13 Rohr (2007), S. 211-212.

14 Z. B. Luff \& Steer (2003); das Erdbebenkapitel existiert auch als redigierte Abschrift in Briefform, BSB, CIm 903.fol.

15 Ovid (1998, 12.521). 
Säugling inmitten grosser Zerstörungen; ${ }^{16}$ ein Thema, das auch in einer süddeutschen und zwei Schweizer Beschreibungen der Jahre 1117, 1356 und 1584 auftaucht. Doch ein Quervergleich mit anderen zeitgenössischen Quellen macht meist deutlich, dass diese Episoden primär mit einer religiös-didaktischen Intention erzählt werden und deshalb höchstens beschränkt eine Aussage über die Intensität des Bebens darstellen.

\subsection{Entwicklung des Erdbeben-Beschreibungsmusters im Lauf der Zeit}

Die annalistische Kurzform einer Erdbeben-Beschreibung mit einem Datum und dem Begriff „terrae motus“ beschränkt sich hauptsächlich auf das Frühmittelalter. Die Kompilatoren führten die Lokalisierung meist behelfsmässig auf das annalenführende Kloster zurück, da sie im Text nicht thematisiert wird ${ }^{17}$. Aussagen zur Lokalisierung sind deshalb oft mit einem gewissen Unsicherheitsfaktor behaftet. Schon die Beschreibungen der Erdbeben am 3. Januar 1117 in Mitteleuropa und Italien und des Erdbebens von Churwalden vom 3. September $1295^{18}$ werden ausführlicher, sie erwähnen neben dem Datum und dem Beben als solchem auch unterschiedliche Beobachtungsorte und Schäden. Breiter wird die Informationsbasis dann in den Chroniken des Spätmittelalters, so vor allem für das Villacher Beben vom 25. Januar 1348,19 dessen Epizentrum heute im Friaul angenommen wird, und für das Basler Beben vom 18. Oktober 1356.20 Diese zwei Ereignisse initiierten wohl die bereits erwähnte erste nachweisbare mitteleuropäische Erdbebenkompilation von Fritsche Closener in Strassburg. ${ }^{21}$

Für das 15. Jahrhundert sind nur wenige kleinere Beben nachweisbar, was wahrscheinlich nicht auf eine schwächere seismische Aktivität zurückzuführen ist, sondern auf mangelhafte Schriftlichkeit und Lücken der Überlieferung. Im 16. Jahrhundert finden sich frühe wissenschaftliche Interpretationsversuche, zum Beispiel zum schwachen Beben von 1531 in der Ostschweiz, das den berühmten Arzt Paracelsus (1493-1541) sogar zu einer Druckschrift veranlasste. ${ }^{22}$ Ein halbes Jahrhundert später fanden die Erdbeben vom 11.-14. März 1584 (gregorianische Datierung) mit dem verheerenden Bergsturz im Gebiet von Aigle dank einem Brief von Johann Rudolf Bullinger und einem Flugblatt von Apiarius vermehrte Beachtung, wobei die nachfolgenden Wellenbewegungen im Genfersee erst heute als Folge einer subaquatischen Rutschung des Rhonedeltas interpretiert werden. ${ }^{23}$ In der sogenannten Wickiana, einer illustrierten Nachrichtensammlung des protestantischen Theologen Johann Jakob Wick (1522-1588) aus den Jahren 1560-1588 in der Zentralbibliothek Zürich, befinden sich einige informative Briefe, die dieses Ereignis beschreiben.

\footnotetext{
16 Sonnabend (1999, S. 50-51).

17 Alexandre (1990, S. 7).

18 Schwarz-Zanetti (2004 und 2008b).

19 Hammerl (1992); Borst (1981).

20 Fäh (2009).

21 Closener (1870), S. 136-137.

22 Paracelsus (1531).

23 Schwarz-Zanetti (2011).
} 
Am Anfang des 17. Jahrhunderts steht das starke Erdbeben von Unterwalden am 18. September 1601, das durch den Universalgelehrten und Stadtschreiber Renward Cysat (1545-1614) von Luzern in einem mehrseitigen Bericht detailliert festgehalten wurde; zwar interpretierte er die zerstörerischen Wellen im Vierwaldstättersee noch als Folge des Bergsturzes vom Bürgenstock, doch die subaquatischen Rutschungen als deren Hauptursache sind heute limnogeologisch nachgewiesen. ${ }^{24}$ In der Mitte des Jahrhunderts nehmen auch die Hinweise auf eine systematischere Beobachtung in kirchlichen Kreisen zu. Die reformierte Kirche des Kantons Zürich scheint von den Gemeindepfarrern briefliche Benachrichtigung über besondere Vorfälle gefordert zu haben. Gleichzeitig wurden zahlreiche schwache Beben in Pfarrbüchern des Kantons Zürich zwischen Taufen, Eheschliessungen und Todesfällen festgehalten, klar datiert und mit unterschiedlicher Ausführlichkeit beschrieben. Solche Notizen erlauben zum Beispiel Rückschlüsse auf den Erdbebenschwarm im Herbst 1650 in der Nordostschweiz, dessen Hauptstoss am 21. September (gregorianische Datierung) in Basel erfolgte und leichte Schäden verursachte.

Die Analyse des Beschreibungsmusters und seiner Entwicklung im Laufe der Zeit stellte Interpretationshilfen für mehrdeutige Texte zur Verfügung. Damit liess sich nicht nur das angebliche Basler Beben von 1021 falsifizieren, sondern es zeigte sich auch, dass das scheinbare Schadenbeben in Süddeutschland vom 3. Januar 1117, das dem grossen Veronese-Beben wenige Stunden vorausging, in seiner Intensitätsbewertung deutlich reduziert werden muss.

\subsection{Das Datenmaterial}

Aus der Spätantike und dem Frühmittelalter liessen sich für das Gebiet der heutigen Schweiz nur wenige Indizien und knappe Beschreibungen von Erdbeben oder damit verbundenen Phänomenen finden (Alexandre 1990, Weiss 1914). Sie sind am Anfang des chronologisch geordneten Katalogteils unter dem Titel „Spuren möglicher und falscher Schweizer Beben des 1. Jahrtausends“ zusammengefasst. Als eigentlicher Ausgangspunkt der Arbeit wurde deshalb das 11. Jahrhundert gewählt, den Endpunkt setzt die Frühaufklärung mit ihrer Ablösung von einem stark religiös geprägten Weltbild und dem Übergang zu einem mehr wissenschaftlichen Denken.

\subsubsection{Jahrhundert}

Da die Schriftlichkeit in diesem Jahrhundert in der Schweiz noch wenig entwickelt war, lassen sich für das 11. Jahrhundert nur vereinzelte und kurze Hinweise auf Erdbeben in den Annalen finden, sie konzentrieren sich vor allem auf die Ostschweiz. Ausserdem erlaubt der in den Quellen meist verwendete Standardsatz „[Datum + ]iterum factum est terrae motus (magnus)“ [Im weiteren geschah am ... ein (grosses) Erdbeben] keine zuverlässige inhaltliche Interpretation.

24 Schnellmann (2004). 
Wichtigstes Resultat der Untersuchung der Quellen des 11. Jahrhunderts ist der genaue Nachweis, wie es im 16. Jahrhundert zur irrtümlichen Annahme eines grossen Schadenbebens im Jahr 1021 in Basel kam. Ausgangspunkt des Irrtums war die Chronik von Johannes Stumpf (1548), der zwar keine Quellenverweise gibt, sich aber mit grosser Wahrscheinlichkeit auf Hypothesen zur Münsterweihe von 1019 stützt, die Rhenanus (1531) und Mutius (1539) vorgetragen hatten, und diese fälschlich mit dem Bayerischen Beben vom 12. Mai 1021 verknüpft. Die Beschreibung des angeblichen Erdbebens selber orientiert sich an einem Bericht von Felix Fabri (1488), gestützt auf den Zeitgenossen Heinrich von Diessenhofen, über das Ereignis von 1356.

Eine quantitative Deutung dieses Überlieferungsbefunds ist schwierig. Mindestens zwei gegensätzliche Aspekte müssen dabei berücksichtigt werden: Zum einen ist die schriftliche Überlieferung aus dieser Epoche im schweizerischen Gebiet noch sehr dünn, so dass es ein seltener Glücksfall ist, wenn ein Ereignis aufgezeichnet und überliefert wurde. Im Weiteren kann man über die Anzahl damals bereits existierender Hochbauten, an denen sich Schäden gezeigt haben könnten, nur mutmassen. Zum andern darf aber aus historischer Sicht mit einer gewissen Wahrscheinlichkeit angenommen werden, dass im 11. Jahrhundert im Gebiet der Nord- und Ostschweiz kein grosses Schadenbeben stattgefunden hat, da es als erschreckendes Naturphänomen und aufgrund der theologischen Bewertung von Erdbeben als Stimme Gottes in den zeitgenössischen klösterlichen Annalen Eingang gefunden haben müsste. Vergleichbar wäre ein derartiges Ereignis auch mit dem Extremwinter 1076/77 in Mitteleuropa, der wegen der wochenlang zugefrorenen Seen und Wasserläufe in zahlreichen Annalen festgehalten worden ist (Schwarz-Zanetti, 1992).

Vertiefte Untersuchungen wären noch nötig, um den Wert der stereotypen Kurzbeschreibungen und der Standardsätze in Quellen des Früh- und Hochmittelalters genauer einzuschätzen. Möglicherweise würde die bereits erwähnte Filiation aller ausgewerteten Annalen, eventuell auch zusätzlicher Annalen, die Abhängigkeitsverhältnisse zwischen einzelnen von ihnen klarer erkennen lassen und unter Umständen weitere scheinbare Originalzeugnisse als Abschriften aus anderen Quellen erweisen, so zum Beispiel für das Ereignis von 1048. Offen blieben oft auch die Fragen zur Bestimmung des Epizentralgebietes von früh- und hochmittelalterlichen Beben.

\subsubsection{Jahrhundert}

Vor allem zwei Faktoren dominieren die Erdbebendaten des 12. Jahrhunderts. Zum einen das grosse Schadenbeben in Verona am 3. Januar 1117, nachmittags ungefähr um $3 \mathrm{Uhr}$, dem wenige Stunden zuvor je ein Beben in Süddeutschland und in der Toskana vorausgingen (Zeller 1913, Alexandre 1990, Guidoboni 2005a, b). Das süddeutsche Erdbeben wurde erst vor kurzem erkannt und als grosses Schadenereignis gewertet. Unterzieht man die betreffenden Zeugnisse allerdings einer Quellenanalyse, so zeigt sich, dass diese Einschätzung mit grosser Wahrscheinlichkeit auf der Fehlinterpretation einiger Quellen beruht. Wie im Katalogteil ausgeführt wird, müssen die Beobachtungen zu diesem Ereignis auf die Intensität 5 zurückgestuft werden.

Zum andern wird der Blick auf das 12. Jahrhundert durch die Annalen von St. Blasien und Engelberg (MGH SS 17, 1861) bestimmt, die ausnehmend häufig Erdbebenbeob- 
achtungen verzeichnen. Im Zeitraum von 1117 bis 1175 werden darin für zehn Jahre insgesamt 13 seismische Beobachtungen festgehalten, und zwar für 1117, 1127, $1128,1134,1155,1158,1161,1162,1170$ und 1175. Nur die Beobachtungen von 1117, 1128 und 1155 können jedoch durch weitere Quellen bestätigt werden. Historisch interessant ist auch die irrtümliche Lokalisierung des grossen syrischen Bebens vom 29. Juni 1170 in Mitteleuropa, nachdem „Sijria“ in den Annalen aus dem steirischen Admont in Österreich (MGH SS 9, 1851) durch einen Abschreibefehler in „Stiria“, also Steiermark, verwandelt wurde. Diese allzu schmale Datenbasis lässt erneut nur bedingt den Schluss auf ein Fehlen von grösseren Schadenbeben in der Schweiz im 12. Jahrhundert zu.

\subsubsection{Jahrhundert}

Auch im 13. Jahrhundert erfasst man nur wenige und meist ungenügend belegte Ereignisse. Der neugefundene Hinweis auf ein Beben im Jahre 1215 aus einem appenzellischen Missale (Von Euw \& Bischofberger 2004) ist zwar interessant, doch als Einzelbeleg nicht gesichert auswertbar. Hinter dem Hinweis auf ein grosses Schadenbeben in Colmar im Jahr 1279 in einer späten Abschrift einer zeitgenössischen Quelle (MGH SS 17, 1861, S. 205) muss mit grosser Wahrscheinlichkeit eine Verwechslung mit dem Basler Beben von 1356 vermutet werden. Am Ende des 13. Jahrhunderts steht das Churwaldner Erdbeben vom 3. September 1295, das als das älteste ausführlicher darstellbare Schweizer Schadenereignis bezeichnet werden kann.

\subsubsection{Jahrhundert}

Die Hinweise in Kompilationen und zeitgenössischen Dokumenten nehmen für das 14. Jahrhundert zu, was vor allem der wachsenden Schriftlichkeit in den Städten in Form von Chroniken zu verdanken ist. Diese Zunahme zeitgenössischer Beobachtungen von Naturereignissen konzentriert sich aber auf die Häufung von Katastrophen in der Mitte des Jahrhunderts, wo nach einer Heuschreckenplage im Jahre 1338 und der Überschwemmung von 1342 in den Jahren 1345-47 eine die Ernten verheerende nasskalte Phase folgte, die mit ihren Hungersnöten den Boden für die anschliessende europaweite Pestepidemie vorbereitete (Schwarz-Zanetti, 1992). Anfangs des Jahres 1348 ereignete sich das grosse Erdbeben von Villach, das heute aufgrund einer neuen Bestimmung des Epizentrums auch als Friaul-Beben bezeichnet wird (Hammerl, 1992). Im Oktober des Jahres 1356 folgte das grosse Basler Beben und im Mai 1357 bei Strassburg ein kleineres Schadenbeben. Die restlichen Hinweise auf Ereignisse in diesem Jahrhundert stammen meist aus erst viel später abgefassten Texten und konnten kaum mit Originalquellen gesichert werden. Viele weitere mussten als Falschmeldung gewertet werden.

\subsubsection{Jahrhundert}

Die Hinweise in den Chroniken und in den Kompilationen nehmen in diesem Jahrhundert erstaunlicherweise ab, was, wie bereits angedeutet, wahrscheinlich weniger mit einer 
sinkenden Erdbebenaktivität zu tun hat als mit der Überlieferung und eventuell mit der schwierigen politischen Situation in der Eidgenossenschaft, welche dem Interesse für die schriftliche Fixierung von Naturereignissen nicht günstig war; jedenfalls lässt sich dieselbe Abnahme der Zeugnisdichte auch bei den Wetterbeobachtungen feststellen (SchwarzZanetti, 1992). Zu einem grossen Teil handelt es sich beim vorhandenen Material um Nachrichten aus später abgefassten Texten, die mit keiner Originalquelle bestätigt werden können, oder um Irrtümer, wie bei dem Meteoritenfall von Ensisheim im Jahre 1492.

\subsubsection{Jahrhundert}

Im 16. Jahrhundert nimmt die überlieferte Datenmenge soweit zu, dass viele Erdbeben als sicher bewertet werden können. Trotzdem bleiben einige Fragen offen. So kann ein möglicherweise durch ein Erdbeben verursachter Turmzusammenbruch in Ardez, GR, im Jahre 1504 durch keinerlei Quellen aus der Umgebung bestätigt werden. Zwei angebliche Beben in den Jahren 1517 und 1523 konnten mit Hilfe von parallelen süddeutschen Wettertagebüchern als Stürme falsifiziert werden. Annalen eines Notars und eine steinerne Inschrift belegen ein Erdbeben im April 1524 im Mittelwallis, das die Kirchtürme von Ardon, Savièse und Conthey (VS) beschädigte.

Umgekehrt sind dank Chroniken aus der Reformationszeit drei Ostschweizer Ereignisse in den 1530 er-Jahren bereits breiter belegt. 1573 findet ein Erdbebenschwarm im Kanton Graubünden mehrfach Aufmerksamkeit. Im Jahr 1577 berichtet dann ein sensationsmässig formuliertes Flugblatt von einem Schadenbeben im Gebiet zwischen Colmar und Bern; nur das Beben, nicht aber die angeblichen Schäden, konnte bisher bestätigt werden. Das Hauptereignis dieses Jahrhunderts ist die Erdbebenserie vom 11.-14. März 1584 mit Schäden im Genferseegebiet, begleitet von einer Flutwelle im Rhonedelta und einem verheerenden Bergsturz oberhalb von Aigle, VD.

Ab 1584 wird in den katholischen Kantonen zunehmend der 1582 von Papst Gregor XIII. eingeführte gregorianische Kalender verwendet, was bis 1701 zu zahlreichen Datierungsfehlern führt - offiziell abgeschlossen wurde dieser Übergang erst 1812; eine ausführlichere Beschreibung dieses Prozesses findet sich im Exkurs zum Kalenderwechsel im Kapitel 2.11.

\subsubsection{Jahrhundert}

Am Anfang des 17. Jahrhunderts steht das grosse Schadenbeben von Unterwalden im Jahr 1601, worüber der Luzerner Gelehrte Renward Cysat den bereits erwähnten Bericht verfasste. Die Datendichte nimmt insgesamt erneut zu, doch reicht es bei schwächeren Beben nicht immer zu gesicherten Aussagen. Besonders zu erwähnen sind das Ftaner Schadenbeben von 1622, der Erdbebenschwarm in der Nordostschweiz von 1650 und ein kleines Schadenbeben in Basel im selben Jahr, sowie die rätselhafte Überschwemmung von 1666 bei Arbon am Bodensee und drei ergänzende Schweizer Beobachtungen zum grossen Beben von Hall, A, im Jahr 1670. 


\subsection{Falschmeldungen}

Bei der Überarbeitung der bestehenden Kompilationen zeigte es sich, dass insgesamt knapp drei Viertel der bisher bekannten Ereignisse unterschiedlicher Korrekturen bedurften. Erwartungsgemäss konzentrierten sie sich auf den in diesem ersten Band behandelten älteren Untersuchungsbereich. Im Folgenden werden einige, teilweise bereits vorgestellte, Datenkorrekturen nach Fehlerursachen gruppiert zusammengestellt.

\subsubsection{Datierungsfehler}

Den grössten Teil machen dabei Datierungsfehler aus, welche die Zeit des bereits erwähnten parallelen Gebrauchs des neuen gregorianischen und des alten julianischen Kalenders in der Schweiz betreffen, also vor allem zwischen 1584 und 1701, in einzelnen Kantonen auch noch länger. ${ }^{25}$ In ECOS-02 und ECOS-09 wurde der Übergang zum gregorianischen Kalender zwecks Vermeidung von Fehlern für alle Erdbeben einheitlich auf 1584 festgelegt, dem Datum des Erstgebrauchs in der Schweiz. In einer Reihe von Fällen führt die Korrektur von Kalenderfehlern dieses Typs lediglich zu einer Verschiebung des Erdbebendatums um zehn Tage und hat für die seismische Auswertung keinerlei Folgen.

Schwerwiegender waren die irrtümlichen Verdoppelungen von Ereignissen aufgrund der unterschiedlich datierenden Quellen aus katholischen und reformierten Kantonen. So wurde zum Beispiel der Beginn der Erdbebenserie vom März 1584 bei Bertrand (1756) und Volger (1857) am 1. und am 10. (sic) März aufgeführt, was im historischen Erdbebenkatalog der Schweiz aus dem Jahre 1999 (MECOS-99) zu einer Verdoppelung der Beben führte. In derselben Weise wurde das Erdbeben vom Herbst 1614 verdoppelt, da Quellen aus dem katholischen Solothurn gregorianisch auf den 4. Oktober datierten, im reformierten Basel aber auf den 24. September. Um denselben Zehntages-Fehler handelt es sich bei den Beben vom 24. Juli und 3. August 1622 und vom 3. und 13. Juni 1642. Noch nicht endgültig entwirrt sind die zahlreichen Daten des Erdbebenschwarms von 1650. Auffallend häufig erscheinen nicht belegbare Ereignisse an runden Daten, wie 1000, 1400 oder 1500, was eher auf ein mystisches Zahlenverständnis als auf reale Erdbeben hindeutet.

Eine weitere Unsicherheit ergibt sich bei Beben in der Nacht, wo ungenaue Uhren und schlaftrunkene Beobachter unterschiedliche Zeitangaben hinterliessen. Beispiele dafür sind in den Quellen über das Beben vom 18. September 1601 um 1 Uhr (UTC) zu finden. Nicht erklärbar sind die zahlreichen leicht unterschiedlichen Daten, die sich mit grosser Wahrscheinlichkeit immer auf das Ereignis vom 1. Juni 1372 beziehen.

${ }^{25}$ Vgl. den Exkurs zum Kalenderwechsel in der Schweiz (Kapitel 2.11). 


\subsubsection{Lokalisierungsfehler}

Lokalisierungen können verschiedenste Fehler enthalten. So sind vermutlich viele zeitgenössische mitteleuropäische Meldungen zum Veronese-Erdbeben von 1117 keine Eigenbeobachtungen, sondern Fremdinformationen von Händlern und Pilgern. Der häufigste Irrtum entsteht jedoch durch nicht gekennzeichnetes Abschreiben fremder Annalen. Es darf also nicht, wie Alexandre postuliert (1990, S. 7), aus dem Fehlen einer expliziten Lokalisierung direkt auf eine lokale Beobachtung am Abfassungsort der Annalen geschlossen werden. Ein spektakuläres Beispiel dafür ist das bereits erwähnte grosse Schadenbeben vom 29. Juni 1170 im syrischen Antiochia (heute das türkische Antakya). Die Fehlinformation mit der irrtümlichen Übertragung in die Steiermark (Sijria wurde zu Stiria) verbreitete sich in ganz Mitteleuropa und damit auch in die Schweiz. Ein ähnliches Missverständnis entstand bei der Erdbebenserie vom März 1584, wo die Schäden am Genfersee irrtümlich an den zürcherischen Greifensee transferiert wurden. ${ }^{26}$

Zwei weitere Lokalisierungsfehler lassen sich für das Beben von 1295 nachweisen. Die zeitgenössischen Annales Basileenses et Colmarienses ${ }^{27}$ orten es im Wallis und in Chur. Eine gleichzeitige Quelle in Osterhofen führt jedoch in glaubwürdiger Form Schäden am Kloster von Churwalden auf, das lateinisch auch Curiovallis genannt wurde. Wahrscheinlich hat der Annalist von Colmar den inm unbekannten Ortsnamen auf zwei ihm besser bekannte Begriffe aufgeteilt. Ein Lesefehler in der Beschreibung des 1295-Bebens in den Annales Seldentalenses (Kloster Seligenthal in Landshut, D) veränderte die Diözese Chur (Churensi) in Chiemsee (Chimensi). Als letztes Beispiel sei ein italienisches Beben von 1152 aufgeführt, das in der Kompilation von Rasch (1591) mit dem damals für Italien gängigen Ausdruck „Welschland“ lokalisiert wurde, einem Begriff, der in der Schweiz seit dem 19. Jahrhundert jedoch mit der Romandie verbunden wird. Im MECOS-99 wurde diese Fehldeutung dann irrtümlich mit „Neuchâtel“ umgesetzt.

\subsubsection{Interpretationsfehler}

Historisch interessant sind komplexe Interpretationsfehler, wie zum Beispiel die bereits erwähnte humanistische Erfindung eines Basler Schadenbebens am 12. Mai 1021 (Schwarz-Zanetti, 2007) in der Chronik von Stumpf (1548). Mit einer Analyse des zeitgenössischen Beschreibungsmusters und Quervergleichen mit Quellen aus derselben Region konnte für das bereits aufgeführte süddeutsche Beben vom 3. Januar 1117 nachgewiesen werden, dass die bisherige Intensitätsschätzung von 8-928 auf 5 reduziert werden muss. Ursache der Fehleinschätzung waren zwei Quellen, die die lokalen Eigenbeobachtungen nicht deutlich gegen die Fremdinformationen zum Schadenbeben von Verona am selben Tag abgrenzen sowie ein phantasievoll ausgeschmückter Bericht mit didaktischer Intention aus Zwiefalten über angebliche Schäden in Augsburg und im Bereich Meersburg-Konstanz.

\footnotetext{
26 Volger (1857), S. 74; auf inn stützte sich der MECOS-99.

27 MGH, SS 17 (1861), S. 183-221.

28 Vgl. Alexandre (1990) und Guidoboni $(2005 a+b)$.
} 
Eine Besonderheit früherer Jahrhunderte sind Verwechslungen mit anderen Phänomenen. Beispielsweise wurden Bergstürze - so derjenige von Plurs im Jahr 1618 oder von Biasca im Jahr 1513 - Erdbeben gleichgesetzt; was durch die wörtliche Bedeutung von „terrae motus“ („Bewegung von Erde“) vielleicht auch abgedeckt werden kann. Erst Quervergleiche zwischen den Quellen ergaben die eigentliche Bedeutung. Im Weiteren konnten einige als Erdbeben verkappte Stürme mit Hilfe von Wettertagebüchern identifiziert werden, so zum Beispiel am 26. Juni 1517 oder am 19. Mai 1523. Auch der Meteoritenfall von Ensisheim im Jahre 1492 wurde aufgrund einer Basler Kopie (Gross, 1624) von Bertrand (1756) und seinen Kopisten als Erdbeben missdeutet.

\subsection{Neue Funde}

Beim bedeutendsten Neufund handelt es sich um ein Schadenbeben im April 1524 in Ardon, VS, mit der maximalen Intensität 8, das Schäden an drei Kirchtürmen und vermutlich auch an Kirchenschiffen verursachte. Eine zeitgenössische Basler Quelle berichtet für den 20. oder 22. April 1524 die Beobachtung eines Bebens in Basel, das aus seismologischen Überlegungen zu diesem Ardon-Beben gehören kann. Daneben wurden einige bisher unbekannte Ereignisse entdeckt, die lediglich als „verspürt“ zu werten waren, wie zum Beispiel das leichte Beben, das wahrscheinlich in Appenzell, sicher aber in der Ostschweiz im Jahre 1215 beobachtet wurde, oder die beiden Beben ohne Schäden von 1402 und 1403, die einem zeitgenössischen Wettertagebuch entnommen werden konnten, dessen Beobachtungen vermutlich in Basel gemacht worden sind. Auch das in Johann Knebels Tagebuch erwähnte leichte Beben in Basel im Jahr 1473 war bisher unbekannt.

Der grösste Teil der neuen Quellenfunde betraf jedoch Ergänzungen zu bereits bekannten Erdbeben, die nur die Kenntnisse vom makroseismischen Feld verbesserten; das herausragendste Beispiel dafür ist das Basler Beben von 1356, dessen Datenqualität mit neuen historischen Quellen und archäologischen Befunden signifikant erhöht werden konnte, ${ }^{29}$ aber auch jenes von 1295 ist hier zu erwähnen.

\subsection{Ausblick}

Betrachtet man die Datenmenge, so fällt erwartungsgemäss ihre ungleiche Verteilung auf der Zeitachse auf. Dies kann für die Anfänge auch mit einer systematischen Suche nur noch punktuell verbessert werden ${ }^{30}$, da die vorhandenen früh- und hochmittelalterlichen edierten Quellen systematisch durchsucht worden sind und Neufunde in unedierten Manuskripten nur noch mit grossem Aufwand gemacht werden können. Eine vertiefte Suche in spätmittelalterlichen Quellen wäre vielversprechender.

\footnotetext{
29 Fäh et al. (2009).

30 Eine Ausnahme ist möglicherweise die Kompilation von Weiss (1914).
} 
Zu erwähnen ist auch das geographische Ungleichgewicht der Daten. Aus dem früher und dichter besiedelten Mittelland sind deutlich mehr Hinweise überliefert als aus den Bergregionen, was vor allem in Gebieten wie dem Wallis, aber auch in der südlichen Zentralschweiz, der Südostschweiz und im Jura ins Gewicht fällt. Im seismisch gefährdeten Wallis bestehen im Mittelalter und in der Frühen Neuzeit noch bedeutende Lücken. Hier könnte die Datenlage möglicherweise mit einer gezielten Suche in den Walliser Kirchenmatrikeln und in den Familienfonds des Walliser Staatsarchivs möglicherweise verbessert werden, wie die Hinweise andeuten, die freundlicherweise von Gregor Zenhäusern und seiner Arbeitsgruppe aus dem laufenden Nationalfonds-Projekt „Klimageschichte des Wallis 1500-1864“31 zur Verfügung gestellt worden sind. Im weiteren werden Dokumente noch genauer $z u$ bearbeiten sein, die den Bau eines Kirchturmes belegen, wie zum Beispiel der Neubau des Kirchturms von Vouvry 1436, der auch 1443 und 1448 bestätigt wird. Im nahegelegenen Vollèges wird im Jahr 1449 eine notwendige Reparatur an der Kirche in einem Testament erwähnt. Oder in einem Prozess zwischen Sembrancher und Vollèges im Unterwallis im Jahre 1665 wird festgehalten, dass die umstrittene Steuer nicht für die Instandsetzung der Kirche und des Kirchturmes zu verwenden sei (StaVS: IMWSZ).

\subsection{Darstellungsschema}

\section{Datum \\ Datierung mit unterschied- licher Genauigkeit}

\section{Zeit (UTC)}

Evt. genaue Zeitangabe in UTC oder Umschreibungen

\section{Ort}

Lokalisierung mit unterschiedlicher Genauigkeit

\section{Bewertung des Ereignisses}

Hier wird die historische Zuverlässigkeit der Rekonstruktion des Gesamtereignisses angegeben: sicher/unsicher/sehr unsicher/falsch, bzw. kein Erdbeben (und gegebenenfalls der Grund dafür [Datierungs-, Lokalisierungs- und Interpretationsfehler beziehungsweise ein anderes Ereignis wie Bergsturz oder Sturm]).

\section{Korrektur(vorschlag)}

Neues Datum, Lokalisierung, andere Ereignisse.

\section{Parameter nach ECOS}

Ix: Maximale Intensität.

\section{Überlieferung}

Nennungen eines Bebens in Texten zweiter Hand (Abschriften, Kopien, Kompilationen).

\footnotetext{
31 Schwarz-Zanetti (2008g).
} 


\section{Quellen}

Liste der zeitgenössischen Beschreibungen und Hinweis auf fehlende Beobachtungen in zeitgleichen Quellen, v. a. in Chroniken und Wettertagebüchern.

\section{Interpretation}

Diskussion der zeitgenössischen Quellen und der Abschriften, ev. Vorschläge für künftige Untersuchungen.

\section{Makroseismische Parameter}

\begin{tabular}{l|l|l|l|l|l|l} 
Ort & Länge & Breite. & $I_{\min }$ & $I_{\max }$ & $I_{\mathrm{w}}$ & Datenqualität
\end{tabular}

Auflistung der Beobachtungsorte mit den jeweiligen Intensitätsschätzungen und der Datenqualität für die einzelnen Orte.

\section{Bemerkungen}

Hier wurden Querverweise usw. eingetragen.

$I_{\min }:$ Minimalwert der Intensität

Imax: Maximalwert der Intensität

Iw: wahrscheinlichste Intensität

\subsection{Exkurs: Der Julianische und der Gregorianische Kalender in der Schweiz ${ }^{32}$}

Die von Papst Gregor initiierte Korrektur des Julianischen Kalenders im Oktober 1582 wurde in der Schweiz ab Ende 1583 in mehreren Schritten regionsweise übernommen, so dass für längere Zeit in zwei verschiedenen Kalenderstilen datiert wurde, die sich bis 1710 um zehn und bis 1838 um elf Tage unterscheiden. Zu den Konsequenzen für die Datierung von Erdbeben vgl. oben 2.7. unter „Datierungsfehler“. Zur genaueren Darstellung der Datierungsproblematik wird im Folgenden ein Teil eines Artikels aus dem Historischen Lexikon der Schweiz zitiert, der als Richtlinie diente. Im Einzelfall sind zur sicheren Beurteilung einer Datierung aber mehrere zeitgenössische Quellen mit erkennbarer Herkunft nötig.

„(...) Am 24.2.1582 [veröffentlichte] Papst Gregor XIII. einen neuen Kalender. Um den Rückstand des bürgerlichen Jahres gegenüber dem astronomischen zu beseitigen, verfügte er die Überspringung von zehn Tagen. Fortan sollte durch das Weglassen dreier Schalttage innert 400 Jahren die Differenz zwischen dem bürgerlichen und dem astronomischen Jahr auf 26 Sekunden reduziert werden. Die sieben katholischen Orte gingen

32 Zwei verschiedene Schreibweisen sind üblich: Julianischer/julianischer und Gregorianischer/gregorianischer Kalender. 
- mit Ausnahme von Ob- und Nidwalden - am 11./22.1.1584 zum neuen Stil über, ebenso das mit Solothurn verbündete neuenburgische Städtchen Le Landeron. Ob- und Nidwalden nahmen den neuen Kalender, nach Überwindung von Widerständen im Volk, einen Monat später an. In der gemeineidgenössischen Vogtei Thurgau führte die Anwendung des neuen Stils 1584 zu Spannungen zwischen Zürich und den fünf innern Orten. Am 6.3.1585 verfügte die Badener Tagsatzung die Feier der kirchlichen Feste nach dem neuen Kalender; die Evangelischen durften jedoch Weihnachten, Stephanstag, Neujahr, Ostern, Christi Himmelfahrt und Pfingsten nach dem alten Stil begehen. Im Appenzellerland führte die Einführung des neuen Kalenders, des sogenannten Gregorianischen Kalenders, zum Widerstand der Äusseren Rhoden. Innerrhoden nahm den neuen Stil 1584 an, Ausserrhoden erst während der Helvetik, an Weihnachten 1798. Im Wallis führten die Bemühungen des Sittener Bischofs Hildebrand von Riedmatten um die Einführung des neuen Kalenders 1584 zum Widerstand der Zenden, die den Verlust alter Bräuche befürchteten. Nach langen Auseinandersetzungen erfolgte am 1./11.3.1656 im ganzen Land der Übergang zum neuen Kalender. Im Untertanengebiet, im Unterwallis, hatte der neue Stil schon 1622 Einzug gehalten. Die evangelischen Stände Zürich, Bern, Basel und Schaffhausen, zudem Katholisch-Glarus, Neuenburg und Genf gingen am 1./12.1.1701 zum neuen Kalender über; die Stadt St. Gallen folgte 1724. EvangelischGlarus nahm den neuen Stil in der Helvetik am 4.7.1798 an. In Graubünden verhinderten das Fehlen einer zentralen Regierung und die konfessionelle Spaltung eine einheitliche Regelung der Kalenderfrage: In den katholischen Gemeinden wurde der Gregorianische Stil 1623-24 eingeführt. In den paritätischen Gemeinden hielten sich von der Mitte des 17. Jahrhunderts an die Katholiken an den neuen Kalender, die Reformierten folgten erst in der 2. Hälfte des 18. Jh. In den evangelischen Gemeinden erfolgte der Übergang zum neuen Kalender zwischen 1783 und 1812: 1783 im Oberengadin und Bergell, in den übrigen Gemeinden später und zu verschiedenen Zeiten. Schiers und Grüsch verweigerten noch 1812, trotz einer Verfügung des Grossen Rats, die Annahme des neuen Stils und wurden deshalb gebüsst. Als letzte Gemeinde in West- und Mitteleuropa nahmen sie 1812 den neuen Kalender an. (...)“

Autor: Hellmut Gutzwiller. [Historisches Lexikon der Schweiz, Teil des Artikels „Kalender“ (http://hls-dhs.dss.ch; 4. August 2008). Abkürzungen wurden hier aufgelöst.]

\section{Übersicht über die Daten des Wechsels vom Julianischen zum Gregorianischen Kalender}

\begin{tabular}{|l|l|}
\hline Region & Datum des Kalenderwechsels \\
\hline $\begin{array}{l}\text { Uri (UR), Schwyz (SZ), Luzern (LU), Zug (ZG), } \\
\text { Fribourg (FR), Solothurn (SO), Le Landeron (NE) }\end{array}$ & $11 . / 22.1 .1584$ \\
\hline Obwalden (OW), Nidwalden (NW) & $11 . / 22.2 .1584$ \\
\hline Thurgau (TG) & 1584 \\
\hline Appenzell Innerrhoden (Al) & 1584 \\
\hline Unterwallis (VS) & 1622 \\
\hline Katholischer Teil Graubündens (GR) & 1623 bis 1624 \\
\hline
\end{tabular}




\begin{tabular}{|l|l|}
\hline Region & Datum des Kalenderwechsels \\
\hline Oberwallis (VS) & $1 . / 11.3 .1656$ \\
\hline $\begin{array}{l}\text { Zürich (ZH), Bern (BE), Basel (BS; BL), Thurgau (TG), } \\
\text { Schaffhausen (SH), Katholischer Teil von Glarus (GL), } \\
\text { Neuenburg (NE), Genf (GE) }\end{array}$ & $1 . / 12.1 .1701$ \\
\hline Stadt St. Gallen (SG) & 1724 \\
\hline Protestantischer Teil Graubündens (GR) & zwischen 1783 und 1812 \\
\hline Appenzell Ausserrhoden (AR) & 1798 \\
\hline Protestantischer Teil von Glarus (GL) & 4.7 .1798 \\
\hline Oberengadin, Bergell (GR) & 1783 \\
\hline Schiers, Grüsch (GR) & 1812 \\
\hline
\end{tabular}

In der Praxis sind abweichende Datierungen verwendet worden. Die heutigen Kantone St. Gallen, Aargau, Tessin und Waadt erscheinen nicht in der Aufzählung, da sie zu dieser Zeit unter Fremdherrschaft standen und nicht als eigenständige Regionen auftraten. 


\section{Erdbebenkatalog der Schweiz 1000-1680}

\section{Spuren möglicher und falscher Schweizer Beben des 1. Jahrtausends}

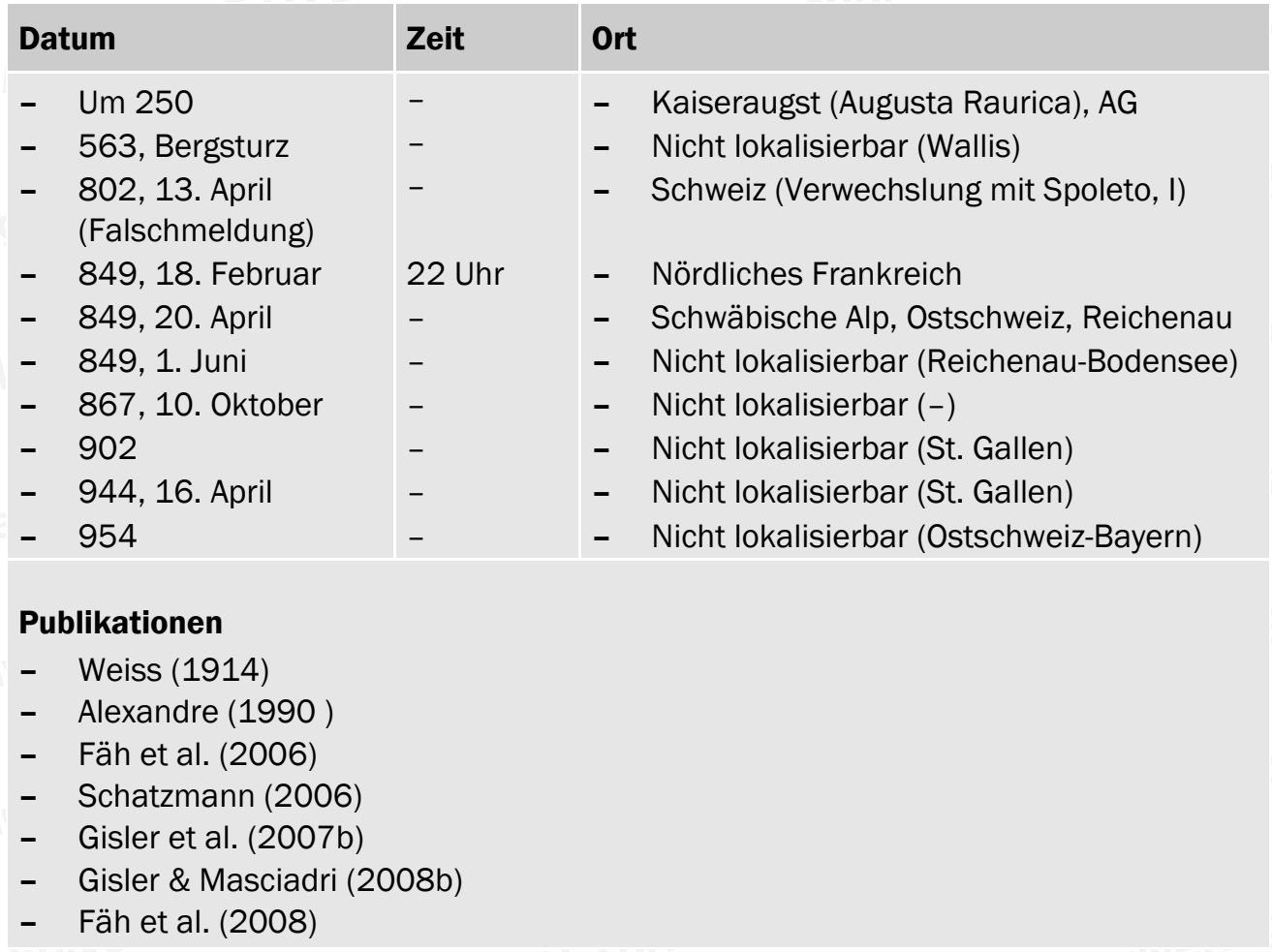

\section{**}

\begin{tabular}{l|l|l} 
Datum & Zeit & Ort \\
$-1000,29$. März & - & - Schweiz \\
-1001 & - & - Schweiz
\end{tabular}

\section{Bewertung der Ereignisse}

Keine Erdbeben: Lokalisierungsfehler. 


\section{Korrektur}

- 29. März 1000 in Flandern

- Kein Korrekturvorschlag für 1001; gehört möglicherweise zu den Falschmeldungen zu einem angeblichen Verona-Beben.

\section{Überlieferung u. a. in:}

Bertrand (1756): Älteste nachweisbare Überlieferung für die Schweiz:

Bertrand (1756, S. 241): „En mille \& un, plusieurs bâtiments furent renversés dans la Suisse par un Tremblement de terre. On y vit aussi des météores ignées, dont les Chroniques parlent comme de quelque chose d'extraordinaire, sans cependant les décrire.“ Bertrand fasst zwei oder mehrere unbekannte Quellen zusammen.

Von Bertrand haben unter anderen abgeschrieben:

- Guéneau de Montbelliard (1761), S. 516: „1001: Tremblement de terre en Suisse, renverse plusieurs maisons, accompagné de plusieurs météores ignés; ... . “

- $\quad$ Hoff (1840-1841)

- Perrey (1843 und 1845)

- Journal de Genève (3. August 1855)

- Volger (1857)

Als Falschmeldungen gewertet:

Alexandre (1990) enthält keine Originalquellen für Erdbeben in der Schweiz in den Jahren 1000 und 1001.

\section{Quellen}

Für die Schweiz 1000 und 1001:

Es konnten keine zeitgenössischen Quellen gefunden werden.

Für Flandern 1000:

Möglicherweise handelt es sich bei Bertrand um eine Verwechslung mit dem südflandrischen Beben vom 29. März 1000, die Quellen dafür sind aufgeführt in Alexandre (1990); Diskussion in Alexandre (1991), S. 49, Punkt e): An unpublished Swiss chronicle of the $16^{\text {th }}$ century, quoted second-hand by Hoff (1840-1841) und Perrey (1845), situated this event in Switzerland [1001], with „destruction of houses“. Diese Interpretation ist aber bereits bei Bertrand (1756) zu finden.

\section{Interpretation}

Es sind bisher keine zeitgenössischen Quellen für ein Schweizer Schadenbeben im Jahre 1001 nachzuweisen. Vielleicht gehört der Hinweis auch zu den Falschmeldungen über ein angebliches Verona-Beben 1001, vgl. Alexandre (1990, S. 192). Ebenso wenig ist beim südflandrischen Beben vom 29. März 1000, mit dem es unter Umständen auch verwechselt worden sein könnte, von Schäden die Rede. Die frühest nachweisbare Schweizer Notiz steht in Bertrands Kompilation (1756); der Irrtum kann bei inm oder in 
seiner unbekannten Vorlage, evtl. die von Hoff (1840-1841) zitierte unpublizierte Schweizer Chronik aus dem 16. Jh., geschehen sein. Vielleicht könnten mentalitätsgeschichtliche Überlegungen zur Bedeutung des Milleniumwechsels eine weitere Erklärung bieten. Bei dieser ungenügenden Quellenlage ist von Falschmeldungen für die Schweiz auszugehen.

\section{Bemerkungen}

Vgl. ECOS-09, in Alexandres Erdbebenkatalog das Jahr 1000 und in seiner Falschbebenliste das Jahr 1001 (1990, S. 137 und 192), ebenso die Falschmeldungen zu den Jahren 1400 und 1500 .

\begin{tabular}{|c|c|c|}
\hline $\begin{array}{l}\text { Datum } \\
\text { 1020, 12. Mai }\end{array}$ & Zeit & $\begin{array}{l}\text { Ort } \\
\text { Bayern (Würzburg), D }\end{array}$ \\
\hline
\end{tabular}

\section{Bewertung des Ereignisses}

Kein Erdbeben: Datierungsfehler.

\section{Korrektur}

12. Mai 1021, nicht lokalisierbar (Bayern-Ostschweiz)

\section{Überlieferung $u$. a. in:}

- $\quad$ Annales Wirziburgenses, 13. Jh. (MGH SS 2, 1829, S. 242)

- $\quad$ Lycosthenes (1557), Basel

- Merian (1834)

- Volger (1857)

- Giessberger (1922)

- Alexandre (1990), Falschbebenliste

\section{Interpretation}

Das Datum 1020 ist mit grösster Wahrscheinlichkeit ein Kopierfehler aus einer der Abschriften der verlorenen Hildesheimer Quelle für das Bayern-Beben 1021 (SchwarzZanetti, 2007). Die Annales Wirziburgenses aus dem 13. Jahrhundert (MGH SS 2, 1829) sind für diesen Schreibfehler die älteste nachweisbare Quelle; bereits in der Edition wird 1021 als Korrektur in Klammern vorgeschlagen. In Lycosthenes, dessen Chronik im Jahre 1557 in Basel gedruckt worden ist, ist derselbe Fehler in der Datierung enthalten, aber ohne dass er eine ausdrückliche Lokalisierung des Bebens vornimmt. Erst in Merian (1834), Volger (1857), Giessberger (1922) und anderen wird in Basel lokalisiert, wahrscheinlich aufgrund des Druckortes Basel der Chronik von Lycosthenes. 


\section{Publikationen}

Nachweis der Fehldatierung in Schwarz-Zanetti et al. (2007) und (2008a).

\section{Bemerkungen}

Vgl. den Eintrag 12. Mai 1021, Bayern-Ostschweiz, Ix = verspürt, und die Falschmeldung vom 12. Mai 1021 in Basel.

\begin{tabular}{|l|l|l} 
Datum & Zeit & Ort \\
1021, 12. Mai & - & Basel
\end{tabular}

\section{Bewertung des Ereignisses}

Kein Erdbeben: Lokalisierungsfehler.

\section{Korrektur}

12. Mai 1021, nicht lokalisierbar (Bayern-Ostschweiz)

\section{Überlieferung u. a. in:}

- Rhenanus (1531)

- Mutius (1539)

- Stumpf (1548)

- Alexandre (1990)

- Gils, Van \& Leydecker (1991)

- MECOS-99

Die meisten jüngeren Kompilationen zitieren dieses angebliche Basler Schadenbeben aus Stumpf oder von dessen Kompilatoren, vgl. die Liste von Alexandre (1990, S. 193), der es bereits wegen fehlender zeitgenössischer Berichte in seine Falschbebenliste verwiesen hat.

\section{Zusammenfassung}

Der Fehler konnte aber detaillierter nachgewiesen werden. Ausgangspunkt dieses in vielen Erdbebenkompilationen vorhandenen Irrtums ist die Chronik von Johannes Stumpf (1548), worin diese Behauptung ohne Quellenverweis aufgeführt ist. Stumpf, oder ein Koautor, stützt sich dabei aber mit grosser Wahrscheinlichkeit auf die Hypothesen zur Basler Münsterweihe von 1019 bei Rhenanus (1531) und Mutius (1539) und verbindet deren Erdbebenhypothese irrtümlich mit dem Beben vom 12. Mai $1021 \mathrm{zu}$ einem Faktum. Die Formulierung der angeblichen Schäden stammt aus einem Bericht von Felix Fabri aus dem Jahre 1488 (gedruckt 1604) über das Basler Ereignis von 1356, der unter anderen den zeitgenössischen Bericht von Heinrich von Diessenhofen (1868) 
verarbeitet hat. Das Datum gehört jedoch zum Erdbeben im Raum Bayern-Ostschweiz vom 12. Mai 1021, das keine nachweisbaren Schäden verursacht hatte.

\section{Publikationen}

- Alexandre (1990)

- Schwarz-Zanetti et al. (2007 und 2008a)

\section{Bemerkungen}

Vgl. den Eintrag 12. Mai 1021, nicht lokalisierbar (Bayern-Ostschweiz), und die Falschmeldung vom 12. Mai 1020.

\section{**}

\begin{tabular}{|l|l|l} 
Datum & Zeit & Ort \\
1021, 12. Mai & - & $\begin{array}{l}\text { Nicht lokalisierbar } \\
\text { (Bayern-Ostschweiz) }\end{array}$
\end{tabular}

\section{Bewertung des Ereignisses}

Gesichertes Erdbeben.

\section{Parameter nach ECOS}

Ix: verspürt

\section{Interpretation}

Aus den Kopien der heute verlorenen Annales Hildesheimenses maiores (kopiert z. B. in den Annales Hildesheimenses minores, (MGH SS 3, 1839, S. 95)) und aus den Annales Sangallenses (MGH SS 1, 1826, S. 82) gibt es ziemlich zuverlässige Informationen zu einem Erdbeben ohne Schäden im Gebiet Bayerns und der Ostschweiz im Jahr 1021. Sie wurden mit der Intensität „verspürt“ gewertet. Interessant wurde dieses Ereignis vor allem aufgrund der nun identifizierten Falschmeldung in der Chronik von Stumpf (1548) über angebliche Schäden in Basel.

\section{Publikation}

- Alexandre (1990)

- Schwarz-Zanetti (2007 und 2008a)

\section{Makroseismische Parameter}

\begin{tabular}{|l|l|l|l|l|l|l|}
\hline Ortschaft & Länge & Breite & $I_{\min }$ & $I_{\max }$ & $I_{w}$ & Datenqualität \\
\hline Bayern & 49 & 12 & 5 & - & verspürt & sehr schwach \\
\hline St. Gallen & 47.43 & 9.4 & 5 & - & verspürt & sehr schwach \\
\hline
\end{tabular}




\section{Bemerkungen}

Vgl. die Falschmeldungen vom 12. Mai 1020, Würzburg, und vom 12. Mai 1021, Basel.

\begin{tabular}{|c|c|c|}
\hline $\begin{array}{l}\text { Datum } \\
\text { 1046, 9. November }\end{array}$ & $\begin{array}{l}\text { Zeit } \\
-\end{array}$ & $\begin{array}{l}\text { Ort } \\
\text { Nicht lokalisierbar } \\
\text { (Süddeutschland/Italien) }\end{array}$ \\
\hline
\end{tabular}

\section{Bewertung des Ereignisses}

Sehr unsicheres Ereignis (Süddeutschland).

\section{Parameter nach ECOS}

Ix: verspürt (Süddeutschland)

\section{Überlieferung u. a. in:}

- Leydecker \& Brünning (1988)

- $\quad$ Alexandre (1990, S. 140 und 223)

- Guidoboni (2005b)

Nicht vorhanden in:

DOM4.1

\section{Quellen}

Zit. nach Alexandre (1990, 140):

- Annales Corbeienses (BrG, 1, Jaffe, 1864, S. 33-43)

- $\quad$ Annales S. Emmerammi Ratisponenses (MGH SS 17, 1861, S. 571-572)

- Annales Ratisponenses, Kopie verlorener Quelle (MGH SS 17, 1861, S. 579-588), eventuell auch Kopie der Annales S. Emmerammi Ratisponenses (MGH SS 17, 1861)

\section{Interpretation}

Dieses sehr unsichere Ereignis wird in den zeitgenössischen Annalen von Corvey, D, mit grossen Schäden im Trentino, I, umschrieben, ohne Beobachtungen in Corvey selber zu erwähnen. Zusätzlich sind aber zwei gleichzeitige Hinweise von Regensburg nachzuweisen; sie könnten ein Indiz für eine lokale Beobachtung sein, denn die Annalen von St. Emmeram und die Annales Ratisponenses sind gemäss Alexandre (1990) original. Wegen der kurzen Notizen lässt sich aber nicht beurteilen, ob die Annales Ratisponenses nicht nur eine Kopie der Annales Sancti Emmerammi sind. Guidoboni (2005b, S. 42) lehnt deren scheinbar lokale Aussagen aber als Quellenkontamination mit italienischen Beobachtungen ab; was eine nicht unwahrscheinliche Interpretation ist. Denn der Informationstransfer zwischen den Klöstern und durch Reisende darf nicht unterschätzt wer- 
den (vgl. das zerstörerische syrische Beben vom 29. Juni 1170, das in vielen europäischen Quellen zuverlässig gemeldet wird).

Überraschenderweise ist das Beben in Italien selber aber nicht nachgewiesen (Guidoboni 2005b, 40-42), ebenso wenig kann der in der Corveyer Quelle erwähnte Fluss Tar im Trentino eindeutig identifiziert werden. Alexandre (1990) schlägt den Eisack vor, Guidoboni (2005b) die Etsch; der Begriff könnte sich unter Umständen auch auf die Drau beziehen. Ausgehend von der Quelle aus Corvey wurde das Beben fälschlicherweise auch nach Norddeutschland transferiert, vgl. Artikel von Leydecker \& Brünning (1988).

Insgesamt handelt es sich um ein äusserst unsicheres Ereignis. Alexandre (1990, S. 223) schätzt die Intensität im Trentino auf 9-10 und in Regensburg auf „inconnue“. Guidoboni (2005b) akzeptiert nur die italienischen Aussagen und bewertet sie mit der Intensität 9.

Hier werden nur die zwei deutschen Quellen berücksichtigt und mit „verspürt“ bewertet. Die Einschätzung der italienischen Information wurde aus der Arbeit von Guidoboni (2005b) übernommen. Während die Lokalisierung beide Sitepunkte umfasst. Mit dieser Datenbasis kann auch ein möglicher Einfluss auf die Schweiz nicht diskutiert werden.

\section{Makroseismische Parameter}

\begin{tabular}{|l|l|l|l|l|l|l|}
\hline Ortschaft & Länge & Breite & $I_{\min }$ & $I_{\max }$ & $I_{w}$ & Datenqualität \\
\hline Regensburg & 49.02 & 12.09 & 5 & & verspürt & sehr schwach \\
\hline Trentino & 46.45 & 11.02 & 7 & 9 & 8 & sehr schwach \\
\hline Hoexter & 51.77 & 9.36 & 1 & 4 & 1 & sehr schwach \\
\hline
\end{tabular}

\begin{tabular}{l|l|l} 
Datum & Zeit & Ort \\
1 1048, 13. Oktober & - & Bayern-Bodensee
\end{tabular}

\section{Bewertung des Ereignisses}

Sicheres Ereignis.

\section{Parameter nach ECOS}

Ix: 5

\section{Überlieferung u. a. in:}

- Lycosthenes (1557), S. 379: 1048. Terremotus magnus factus est circa Constantiam ad lacum Acroniam sitam 3. Idus Octobris (13. Oktober). (Am 13. Oktober 1048 ereignete sich ein Erdbeben in der Gegend von Konstanz, am Bodensee.) [Vermutlich stammt die Information aus der Chronik von Hermann von Reichenau, die bereits 1529 gedruckt worden war.] 
- $\quad$ Hoff (1840-1841)

- Perrey (1845)

- $\quad$ Volger (1857)

- Sieberg (1940)

- Montandon (1942-43)

- Alexandre (1990)

- MECOS-99

\section{Quellen}

\section{Originaltexte:}

- Herimanni Augiensis Chronicon (1844, MGH SS 5, S. 67 f.): Quibus diebus terrae motus magnus factus est nocte 3. Idus Octobris. [Ein grosses Erdbeben hat sich in diesen Tagen in der Nacht auf den 13. Oktober ereignet (in Reichenau).] Hermann von Reichenau (1013-1054) war Zeitzeuge.

- Annales S. Emmerammi Ratisbonenses et Weltenburgenses (MGH SS 17, 1861, S. 572): Terrae motus factus est magnus 3. Idus Octobris. [Am 13. Oktober ereignete sich ein grosses Erdbeben; in Regensburg.] Die Abtei Weltenburg liegt in Kelheim an der Donau, $20 \mathrm{~km}$ südwestlich von Regensburg.

- Annales Altahenses Maiores (1891, 1997, MGH SRG 4): Terrae motus IIII: Id. Octobris (factus est). [Am 12. Oktober ereignete sich ein Erdbeben (in Altaich, OSO von Regensburg).]

\section{Abschrift, Original verloren:}

- $\quad$ Annales Ratisponenses (1861, MGH SS 17. S. 577 f.): 1048. Terremotus pridie Idus Octobris. [Erdbeben am Tage vor dem 15. Oktober (in Regensburg).]

Nicht vorhanden in:

- $\quad$ Annales Einsidlenses (MGH SS 3, 1839, S. 137)

- Annales Frisingenses (MGH SS 13, 1881, S. 51-60)

\section{Interpretation}

Trotz leicht unterschiedlicher Datierungen scheint es sich mit grosser Wahrscheinlichkeit um ein Einzelbeben in der Nacht vom 12. auf den 13. Oktober 1048 im Raum BayernBodensee zu handeln (Alexandre, 1990). Die Intensität wird zweimal mit dem Begriff „magnus“ umschrieben. Hinter diesen knappen hochmittelalterlichen Formulierungen können sich zwar durchaus (kleinere) Schäden verstecken, ebenso gut können hier der Schrecken und eine theologische Interpretation von Erdbeben eine gewichtige Rolle spielen. Vor allem aber sollen Intensitätsbeschreibungen ohne Schadensbeschreibungen höchstens mit Ix $=5$ bewertet werden. Dass in den Annales Einsidlenses und in den Annales Frisingenses dieses Erdbeben nicht erwähnt wird, könnte ein Indiz für ein eher lokal beschränktes Ereignis sein.

Die grosse Ähnlichkeit der Beschreibungen kann einerseits von der kurzen und standardisierten hochmittelalterlichen Formulierung stammen, andererseits kann sie auch ein Zeichen für Abschriften sein. Die Abhängigkeiten innerhalb dieser vier Quellen konnten 
jedoch noch nicht genauer untersucht werden. Deshalb wird hier dieses Ereignis aufgrund der drei Originale und einer Abschrift nur vorläufig als „gesichert“ und mit einer Intensität Ix $=5$ gewertet.

Makroseismische Parameter

\begin{tabular}{|l|l|l|l|l|l|l|}
\hline Ortschaft & Länge & Breite & $I_{\min }$ & $I_{\max }$ & $I_{w}$ & Datenqualität \\
\hline Niederaltaich & 48.83 & 13 & 3 & & verspürt & sehr schwach \\
\hline Lindau im Bodensee & 47.56 & 9.7 & 4 & 5 & 5 & sehr schwach \\
\hline Reichenau, Bodensee & 47.7 & 9.06 & 4 & & 5 & sehr schwach \\
\hline Regensburg & 49.02 & 12.09 & 4 & & 5 & sehr schwach \\
\hline
\end{tabular}

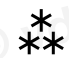

\begin{tabular}{l|l|l} 
Datum & Zeit & Ort \\
1048, 15. und & - & Konstanz, D \\
16. Oktober & &
\end{tabular}

\section{Bewertung des Ereignisses}

Keine Erdbeben: Datierungsfehler.

\section{Korrektur}

1048, in der Nacht vom 12. auf den 13. Oktober

\section{Überlieferung u. a. in:}

- Lycosthenes (1557)

- Perrey (1845)

- Volger (1857)

- Montandon (1942-43)

- MECOS-99

\section{Interpretation}

Diese Fehldatierungen stammen von Volger (1857), der sich bei den Datierungen 13., 15. und 16. Oktober 1048 über Perrey (1845) angeblich auf Lycosthenes (1557) stützt, letzterer erwähnt jedoch nur den 13. Oktober. Was auch durch die drei Originaltexte und die Abschrift einer verlorenen Quelle als richtig bestätigt wird.

\section{Bemerkungen}

Vgl. den vorangehenden Eintrag für das Ereignis am 13. Oktober 1048.

$$
\text { *** }
$$




\begin{tabular}{|l|l|l}
\hline Datum & Zeit & Ort \\
1051 & - & Breisgau, D
\end{tabular}

\section{Bewertung des Ereignisses}

Kein Erdbeben.

\section{Überlieferung u. a. in:}

- Sieberg (1940): Erdbeben im Breisgau. Verweist auf seine eigene Arbeit „Erdbebenkatalog der preussischen Rheinlande, umfassend die Jahre 600-1895. Handschriftlich, bearbeitet um 1900 herum, ... (Original im GFZ)“. Er habe dem Aachener Stadtarchivar Pick den Nachweis wertvoller, sonst unbekannter Quellen zu verdanken. Von letzterem seien neben historischen Sammelwerken und Gelegenheitsschriften bloss folgende Ortschroniken erwähnt: Handschriftliche Chroniken und Kirchenbücher von Kloster Attendorn, von Cornelimünster, Dortmund, Freihaldenhoven, Maastricht, Schwerfen, Stavelot und Winningen; ferner gedruckte Berichte und Chroniken von Aachen, Erkelenz, Eschweiler, Kloster Frauweiler, Kloster Kamp, Köln, Malmedy, Mayschoss an der Ahr, Münstereifel, Kloster Rolandswerth und Zülpich.

- Rothé \& Schneider (1968): Catalogue des Tremblements de terre du Fossé Rhenan. 1968. Diese stützen sich wiederum auf Sieberg (1940).

- MECOS-99, stützt sich auf Rothé \& Schneider (1968) sowie auf Sieberg (1940).

Falschmeldung:

Alexandre (1990) kennt keine zeitgenössischen Hinweise, weshalb er es in seiner Liste der falschen Beben aufführt.

\section{Interpretation}

Da Alexandre (1990) dieses Erdbeben in seiner Falschbebenliste aufführt und erst Sieberg (1940) von einem Breisgauer Beben ohne Schaden im Jahr 1051 spricht, ohne seine Quelle anzugeben, so ist vorläufig von einer Falschmeldung auszugehen.

\section{Bemerkungen}

Vgl. den Eintrag den Eintrag in der Fakeliste im ECOS-09; kein Korrekturvorschlag.

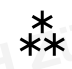

\begin{tabular}{|l|l|l|}
\hline Datum & Zeit & Ort \\
1062, 8. Februar & - & $\begin{array}{l}\text { Nicht lokalisierbar } \\
\text { (Reichenau, D) }\end{array}$ \\
\hline
\end{tabular}

\section{Bewertung des Ereignisses}

Unsicheres Ereignis. 


\section{Parameter nach ECOS}

Ix: verspürt

\section{Überlieferung u. a. in:}

- Lycosthenes (1557)

- Bertrand (1756)

- Merian (1834)

- Hoff (1840-1841)

- Journal de Genève (1855)

- Volger (1857): Über Merian (1834) zitiert er irrtümlicherweise Hermannus von Reichenau als Quelle. Doch es muss sich um die Fortsetzung des „Herimanni Augiensis Chronicon“ von Bertholdus handeln, denn Hermannus lebte nur bis 1054 (Bertholdi Annales, 1844).

- MECOS-99

- Alexandre (1990), mit Verweis auf Originalquelle

\section{Quelle}

Bertholdi Annales (MGH SS 5, 1844, S. 264-326):

1062 (...) 6. Idus Februarii (8. Februar) terraemotus, fulgura et tonitrua facta sunt. [Am 8. Februar 1062 geschahen ein Erdbeben, Blitze und Donner.]

\section{Interpretation}

Wegen einer Fehlinterpretation für das Jahr 1092 wird dieses unsichere Ereignis hier vorgestellt. Bisher ist nur diese eine Originalquelle von der Insel Reichenau im Bodensee nachweisbar; Alexandre (1990) hält sogar eine Verwechslung mit dem darin erwähnten Gewitter möglich. (Vgl. die eindeutig nachgewiesenen Verwechslungen mit Stürmen am 26. Juni 1517 und am 19. Mai 1523; und die nicht ganz gesicherte Verwechslung am 17. November 1533.) Mehrere Kompilationen führen auch ein Erdbeben am 8. Februar 1092 in Konstanz auf, ohne einen zeitgenössischen Beleg zu nennen; sodass dort wegen der Ähnlichkeit des Datums und der Lokalisierung von einem Schreibfehler für 1062 ausgegangen werden muss.

Die Intensität wird in der Quelle von Bertholdus nicht umschrieben, sodass sie nur mit „verspürt“ gewertet werden kann.

Weitere Arbeiten zu diesem Ereignis:

- Wolf (1989 und 1993)

- Schmedes et al. (1992)

Makroseismische Parameter

\begin{tabular}{|l|l|l|l|l|l|l|}
\hline Ortschaft & Länge & Breite & $I_{\min }$ & $I_{\max }$ & $I_{w}$ & Datenqualität \\
\hline Reichenau, Bodensee & 47.7 & 9.06 & & & verspürt & sehr schwach \\
\hline
\end{tabular}




\section{Bemerkungen}

Vgl. die Falschmeldung am 8. Februar 1092; evt. gehört diejenige von 1098 auch zu diesem Ereignis.

\begin{tabular}{|c|c|c|}
\hline $\begin{array}{l}\text { Datum } \\
\text { 1081, 27. März }\end{array}$ & $\begin{array}{l}\text { Zeit } \\
-\end{array}$ & $\begin{array}{l}\text { Ort } \\
\text { Schweiz }\end{array}$ \\
\hline
\end{tabular}

\section{Bewertung des Ereignisses}

Kein Erdbeben: Lokalisierungsfehler.

\section{Korrektur}

Belgien, 27. März 1081

\section{Überlieferung u. a. in:}

- Lycosthenes (1557)

- $\quad$ Hoff (1840-1841)

- Perrey (1845)

- Nöggerath (1870)

- Giessberger(1922)

- $\quad$ Sieberg (1940)

Belgische Hinweise:

- Alexandre (1990)

\section{Interpretation}

In Lycosthenes (1557, S. 383) steht der älteste bekannte Schweizer Hinweis auf das Ereignis vom 27. März 1081, und zwar ohne Schadensbeschreibungen und ohne ausdrückliche Lokalisierung. Alexandre (1990, S. 143) hingegen enthält vier Originalhinweise auf ein belgisches Beben dieses Datums. Die Formulierung von Lycosthenes wiederum deutet mit grosser Wahrscheinlichkeit auf eine Abschrift aus dem Chronicon von Sigebert von Gembloux (vgl. Alexandre 1990) hin, das bereits 1513 gedruckt worden war.

\section{Bemerkungen}

Vgl. den Eintrag in der Fakeliste des ECOS-09 für den 27. März 1081.

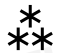




\begin{tabular}{|l|l|l|}
\hline Datum & Zeit & Ort \\
\hline $1092,8$. Februar & - & Konstanz, D \\
\hline
\end{tabular}

\section{Bewertung des Ereignisses}

Kein Erdbeben: Datierungsfehler.

\section{Korrektur}

8. Februar 1062

\section{Überlieferung u. a. in:}

- Beuther (1557)

- Hoff (1840-1841)

- Perrey (1845)

- Volger (1857)

- MECOS-99

\section{Interpretation}

Es sind bisher keine zeitgenössischen Belege für den 8. Februar 1092 nachweisbar. Zusätzlich lässt die Ähnlichkeit des Datums mit dem nicht ganz gesicherten Erdbeben vom 8. Februar 1062 mit grosser Wahrscheinlichkeit den Schluss auf einen paläografischen Irrtum zu.

\section{Bemerkungen}

Vgl. das Erdbeben vom 8. Februar 1062; möglicherweise gehört das angebliche Ereignis von 1098 auch dazu.

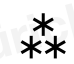

\begin{tabular}{|l|l|l}
\hline Datum & Zeit & $\begin{array}{l}\text { Ort } \\
\text { Nicht lokalisierbar } \\
\text { (Schaffhausen) }\end{array}$ \\
\hline
\end{tabular}

\section{Bewertung des Ereignisses}

Unsicheres Ereignis.

\section{Parameter nach ECOS}

Ix: 3

\section{Überlieferung}

Alexandre (1990) 


\section{Quelle}

Bernoldi Chronicon (MGH SS 5, 1844, S. 455): „Magnus quoque terrae motus in eodem episcopatu apud cellam Salvatoris eo tempore noctu contigit; unde et paucis tunc innotuit, praeter quosdam religiosos viros et feminas quibus eadem nocte nondum somnus obrepsit." [Zu jener Zeit hat sich auch ein grosses Erdbeben beim Kloster Allerheiligen im genannten Bistum (d. h. Konstanz) ereignet, und zwar nachts; deshalb haben es damals nur wenige bemerkt, ausser einigen Mönchen und Nonnen, die in jener Nacht noch nicht schliefen.]

\section{Interpretation}

Es handelt sich um ein Ereignis, das lediglich von wenigen Mönchen und Nonnen im Kloster Allerheiligen in Schaffhausen verspürt worden ist. Gemäss Alexandre (1990) könnte es sich bei dieser Einzelbeobachtung sogar um eine politisch beeinflusste Interpretation im Zusammenhang mit dem Investiturstreit handeln. Trotz zeitgenössischer Quelle muss deshalb dieses Ereignis als „unsicher“ und mit der Intensität 3 gewertet werden.

Die Tatsache, dass das angeblich grosse Erdbeben die Schlafenden nicht geweckt hat, ist auch ein Beispiel dafür, dass bei der Auswertung von mittelalterlichen IntensitätsAdjektiven Vorsicht geboten ist.

\section{Makroseismische Parameter}

\begin{tabular}{|l|l|l|l|l|l|l|}
\hline Ortschaft & Länge & Breite & $I_{\min }$ & $I_{\max }$ & $I_{\text {w }}$ & Datenqualität \\
\hline Schaffhausen & 47.7 & 8.64 & 2 & 4 & 3 & sehr schwach \\
\hline
\end{tabular}

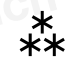

\begin{tabular}{l|l|l} 
Datum & Zeit & Ort \\
1098 & - & Reinach-Basel
\end{tabular}

\section{Bewertung des Ereignisses}

Kein Erdbeben: eventuell ein Datierungsfehler.

\section{Korrektur}

Eventuell 8. Februar 1062

Überlieferung u. a. in:

- Merian (1834)

- Perrey (1845)

- $\quad$ Volger (1857)

- MECOS-99 


\section{Quellen}

Es sind bisher keine zeitgenössischen Quellen nachweisbar.

\section{Interpretation}

Sehr unsicheres, wahrscheinlich sogar falsches Ereignis, denn die Information kann nur bis Merian (1834) zurückverfolgt werden. Dieser habe Wurstisen (1580) zitiert, der sich seinerseits auf Schriften von Harscher gestützt habe: „ex libro Harscheri“. In Wurstisen ist jedoch kein Hinweis auf 1098 zu finden, sodass Merian sich mit dem Quellenverweis möglicherweise geirrt hat. Denn aus jüngerer Zeit lässt sich ein Nikolaus Harscher (1683-1742) in Basel nachweisen, der historische Arbeiten verfasst hat, was noch weiter zu überprüfen wäre. Als reine Spekulation sei hier noch eine Verwechslung mit dem Erdbeben von 1062 vorgeschlagen.

\section{Bemerkungen}

Vgl. den Eintrag im ECOS-09 zum 8. Februar 1062 und die Fakeliste des ECOS-09 zum Jahr 1092.

\section{$\stackrel{*}{* *}$}

\begin{tabular}{l|l|l}
\hline Datum & Zeit & Ort \\
1107 & - & Süddeutschland
\end{tabular}

\section{Bewertung des Ereignisses}

Kein Erdbeben: Datierungsfehler.

\section{Korrektur}

1117 , 3. Januar

\section{Überlieferung u. a. in:}

- Wolfart (1909/1979, Bd. 2, S. 278)

- Giessberger (1922): „Erdbeben in Lindau“

- Sieberg (1940): „1107. Grosses Erdbeben, wohl in Süddeutschland. Ausser in Lindau anscheinend gefühlt in Strassburg, Marbach und Bamberg." Quellen sind das Manuskript von Lersch (GFZ, das nicht überprüft werden konnte), und Giessberger (1922).

- MECOS-99

Nicht nachgewiesen u. a. in:

- Volger (1857)

- Candreia (1905)

- Wolfart (1912) 
Abgelehnt:

In Alexandre (1990) wird dieses Ereignis in der Falschbebenliste aufgeführt, weil keine zeitgenössischen Quellen nachgewiesen werden konnten.

\section{Interpretation}

Abgesehen davon, dass Alexandre (1990) keine zeitgenössischen Beobachtungen nachweisen kann, ist dieses Erdbeben auch nicht in der späteren Erdbebenchronik von Wolfart (1912) vorhanden. Wegen der Ähnlichkeit des Datums von 1107 mit 1117 handelt es sich vielmehr mit grösster Wahrscheinlichkeit um eine Verwechslung mit dem süddeutschen oder mit dem Veronese-Erdbeben vom 3. Januar 1117.

\section{Bemerkung}

Vgl. das süddeutsche und das Veronese-Erdbeben vom 3. Januar 1117.

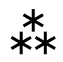

\begin{tabular}{|l|l|l}
\hline Datum & Zeit & Ort \\
\hline 1112, 3. Januar & - & Rottenburg am Neckar, D
\end{tabular}

\section{Bewertung des Ereignisses}

Kein Erdbeben: Datierungsfehler.

\section{Korrektur}

1117, 3. Januar

\section{Überlieferung u. a. in:}

Älteste Überlieferung:

- Flores temporum Auctore Fratre Suevico ordinis minorum (MGH SS, 24, 1879, S. 230-250): „(1112). Terre motus per totum orbem magnas et multas subruit urbes; terra quoque dehiscens in pluribus locis civitates cum suis habitatoribus absorbuit 3. Non. Jan.“ [Ein grosses Erdbeben in der ganzen Welt hat am 3. Januar 1112 viele grosse Städte zum Einsturz gebracht; auch hat die Erde sich aufgetan und an mehreren Orten Städte mit ihren Bewohnern verschlungen.]

- Lycosthenes (1557, S. 397): „1112. Rotenburgium civitas iuxta Neccarum, terraemotu concidit, ... . “[1112. Die Stadt Rottenburg am Neckar ist infolge eines Erdbebens eingestürzt, .... .]

- Zeller (1913, S. 267 f.) geht der Überlieferung zum Erdbeben und einer gleichzeitigen Überschwemmung historisch-kritisch nach: Chronik des Horber Bürgermeisters Besenfelder um 1450, Johannes Nauclerus um 1500, Steininschrift in Rottenburg, usw. 
Nach Lycosthenes zitieren unter anderen:

- Hoff (1840-1841)

- Perrey France (1843)

- Perrey Rhin (1845)

- Nöggerath (1870)

- Giessberger (1922)

- Lancaster (1901)

- Van Rummelen (1945)

1112 abgelehnt durch:

- Zeller (1913)

- Alexandre (1990)

\section{Interpretation}

Die Flores Temporum, die Ende 13. Jahrhundert wahrscheinlich in Reutlingen (nahe von Rottenburg gelegen) entstanden und bis ins 15. Jahrhundert häufig abgeschrieben wurden, irren sich im Jahres-Datum. Ein Fehler, der auf unbekanntem Weg auch in Lycosthenes (1557) auftaucht, lokalisiert in Rottenburg, und danach oft kopiert worden ist. Obwohl Lycosthenes keine Präzisierung des Datums mit einer Tagesbezeichnung gibt, so ist auch bei inm aufgrund der Formulierung anzunehmen, dass 1112 mit 1117 verwechselt worden ist, genauso wie die dort erwähnte Überschwemmung in Lüttich mit grosser Wahrscheinlichkeit auf 1117 zu verschieben ist (Schwarz-Zanetti, 1992). Ursache ist wahrscheinlich ein Lesefehler, denn in mittelalterlichen Handschriften können die Zahlen "2“ und „7“ eine sehr ähnliche Form haben (vgl. Cappelli, 1901, S. 439 und 441).

Eine heute nicht mehr nachweisbare Steininschrift in Rottenburg kann eine zusätzliche Rolle gespielt haben. Zeitgenössische Hinweise auf eine Zerstörung Rottenburgs im Jahre 1117 fehlen ebenso.

\section{Bemerkung}

Vgl. das süddeutsche Erdbeben vom frühen Morgen des 3. Januar 1117 und das Veronese-Beben vom Nachmittag desselben Tages.

\section{***}

Datum

1115
Zeit

$-$
Ort

Zürich

\section{Bewertung des Ereignisses}

Kein Erdbeben: Datierungsfehler.

\section{Korrektur}

1117, 3. Januar 


\section{Überlieferung u. a. in:}

- Lindiner (1711)

- $\quad$ Ebel, Manuskript im StAZH: B IX (zwischen 1805 und 1830 beendet)

- $\quad$ Volger (1857)

- Sieberg (1940); datiert auf den 15. Juli, basierend auf dem hier nicht überprüften Manuskript von Lersch (GFZ).

Abgelehnt durch:

Alexandre (1990), Falschbebenliste

\section{Quellen}

Keine zeitgenössischen Quellen nachweisbar.

\section{Interpretation}

Einerseits datiert Sieberg (1940) auf den 15. Juli 1115, was nicht mit zeitgenössischen Hinweisen bestätigt werden konnte. Andererseits lässt die kurze Bemerkung in Lindiner (1171, S. 70-71) auch hier auf eine Verwechslung mit 1117 schliessen: „Erdbidem. Waren gespürt gar empfindlich A. 1115. (...)“

\section{Bemerkungen}

Vgl. das süddeutsche Erdbeben vom frühen Morgen des 3. Januar 1117 und das Veronese-Beben vom Nachmittag desselben Tages.

\section{**}

\section{Datum}

- 1117, 3. Januar

- $[-1117,3$. Januar

- 1117, 3. Januar

\section{Zeit (UTC)}

- 1 Uhr

- Nachmittag, 1300 Uhr

- Nachmittag, $1300 \mathrm{Uhr}$
Ort

- Süddeutschland

- Veronese-Beben (Italien bis Zentraleuropa)

- Toskana]

\section{Bewertung des Ereignisses}

Sichere(s) Ereignis(se).

\section{Parameter nach ECOS}

- Süddeutschland-Erdbeben Ix: 5

(- Veronese-Erdbeben Ix: 9)

\section{Überlieferung u. a. in:}

Bisher wurde unter dem Datum 3. Januar 1117 in den Chroniken und in den meisten Kompilationen nur das sogenannte „Veronese-Beben“ vom Nachmittag verstanden, so in Lycosthenes (1557), Bertrand (1756), Volger (1857), Barrata (1901), Sieberg (1940) und 
anderen; der MECOS-99 jedoch führte dazu nur das falsche Datum „1107“ auf. Alexandre (1990) interpretierte es dann als italienisch-mitteleuropäisches Schadenbeben und Guidoboni etal. (2005a,b) schlugen ein dreiteiliges Erklärungsmodell mit Schäden in Süddeutschland vor.

\section{Quellen}

Auswertungen mit Listen von zeitgenössischen Quellen:

- Zeller (1913); er fand bisher in der Forschung keine Beachtung.

- Guidoboni (1984, 2005a und 2005b)

- Alexandre (1984 und 1990)

Fehl- bzw. Zusatzinterpretationen für die Schweiz:

- Gemäss Moser (1998, S. 294) ist der angebliche Zusammensturz der Burg Fenis durch das Erdbeben von 1117, wie er zum Beispiel auch von Annemarie Dubler im Historischen Lexikon der Schweiz (http://hls-dhs-dss.ch, 22. September 2008) angenommen wird, eine Erfindung von Jonas Boyve.

- Boyve (1854-1858)

- Es ist noch zu überprüfen, ob die Arbeiten von Känel (1980) und Gutscher (1997) mit Schadensvermutungen auf der Petersinsel zu 1117 unabhängig von der FenisBehauptung von Boyve sind.

\section{Neuinterpretation des Erdbebens vom 3. Januar 1117 in Süddeutschland}

Die Erdbeben vom 3. Januar 1117 sind vom SED für den Erdbebenkatalog des Jahres 2002 (ECOS-02) nicht ausgewertet worden, wie Guidoboni (2005a) irrtümlich annimmt, vielmehr wurden nur die italienischen Daten (DOM4.1, 1997) für das Verona-Beben übernommen. Da das Gebiet der Schweiz aber von zwei Beben mitbetroffen war, wie Notizen aus Einsiedeln und aus mehreren schweiznahen Orten, wie St. Blasien im Schwarzwald, Konstanz, Zwiefalten, Marbach, Bamberg, Augsburg, Regensburg und Melk belegen, wird im Folgenden eine ausführliche Neubewertung der nordalpinen Schriftquellen zu den Ereignissen am frühen Morgen des 3. Januars 1117 vorgestellt.

Die bisherigen Positionen zum süddeutschen Erdbeben sind kontrovers. Zum einen bezweifelte bereits Sieberg (1940) die angeblichen Schäden in Mitteleuropa, und Hülsen (1993) vermutet sogar eine generelle Kontamination der mitteleuropäischen Quellen mit norditalienischen Fremdinformationen. Zum anderen geht Alexandre (1990, S. 232) von einem einzigen riesigen Schütterbereich von Norditalien bis Norddeutschland aus und deutet mit einem graphischen Symbol eine Intensität von 8-9 an, ohne auf die bereits von ihm erkannten zwei Stösse vertiefter einzugehen. Guidoboni (2005a, b) ihrerseits teilt das gesamte Geschehen in drei unabhängige Beben auf, wovon das erste sich am frühen Morgen mit Epizentrum in Augsburg $\left(48.00^{\circ} \mathrm{N}, 9.42^{\circ} \mathrm{E}\right)$ ereignete habe, dessen maximale Intensität 8-9 betrage bzw. ein Magnitude 6.4 habe.

Als historische Kernfragen erweisen sich unter diesen Voraussetzungen das Erkennen von Fremdinformationen und Abschriften, die Bestimmung der Uhrzeit, die genaue Lokalisierung der Informationen, vor allem der Schäden, die Distanz zwischen Ereignis und Niederschrift sowie die religiös-kulturellen Aspekte der Quellen. 


\section{Zuverlässige Datierung und Uhrzeit}

Dank der Quellenarbeiten von Zeller (1913), Alexandre $(1984,1990)$ und Guidoboni (1984 und 2005a, b) ist zumindest die Tagesdatierung gesichert. Zusätzlich erwähnen neun der vierzig bekannten Quellen aus Mitteleuropa ein Vorbeben in Süddeutschland, und zwar vor Tagesanbruch, wahrscheinlich um den Hahnenschrei, der um 2 Uhr anzusetzen ist, also 1 Uhr UTC, und einen zweiten Stoss um die Vesperzeit des 3. Januar 1117. Während die italienischen Quellen, mit Ausnahme von Pisa (Guidoboni, 2005b, S. 126 f.), von einem einzigen Beben um die Vesperzeit berichten (Alexandre 1990, S. 154).

Die Niederschrift aller neun Quellen [Annales S. Disibodi (MGH SS, 17, 1861, S. 6-30), Annales S. Stephani Frisingensis (MGH SS 13, 1881, S. 51-60), Annales Augustani minores (MGH SS 10, S. 8-11), Casus monasterii Petrishusensis (MGH, SS, 20, 1869, S. 621-683), Annales S. Blasii in Silva Nigra (MGH SS 17, 1861, S. 275-282), Annales Zwifaltenses (MGH SS 10, 1852, S. 53-64), Annales S. Rudberti Salisburgensis (MGH, SS, Bd. 9, 1851, S. 758ff.), Auctarium Zwetlense (MGH SS 9, 1851, S. 540) und Annales et Notae Schaeftlarienses (MGH SS 17, 1861, S. 334-342)] hat noch im 12. Jahrhundert stattgefunden. Doch meistens ist die Distanz zwischen Ereignis und Niederschrift so gross, dass sie eher als Abschriften gewertet werden müssen. Deren Zuverlässigkeit soll im folgenden überprüft werden.

Von den neun süddeutschen Quellen nennen sieben das Jahr 1117; zwei datieren irrtümlicherweise mit 1116, was auf eine gewisse Distanz zwischen Ereignis und Niederschrift sowie gleichzeitig auf eine Abschrift deutet. Der Tag wird von acht Quellen übereinstimmend auf den 3. Januar festgelegt, siebenmal mit der Formulierung „3. Non. lan.“, einmal „mit Octava sancti lohannis ewangelistae“. Die Uhrzeit zeigt dann grössere Variationen in der Formulierung, denn sechs Quellen sprechen von der Zeit vor Tagesanbruch und von der Vesper, drei nennen aber die Reihenfolge „Tag-Nacht“. Die sechs Quellen, die ein Beben vor Tagesanbruch postulieren, stimmen inhaltlich überein, zeigen aber deutliche Unterschiede in der Formulierung, was mit einer grossen Wahrscheinlichkeit auf eine originale Beobachtung oder auf Abschriften von unterschiedlichen Originalbeobachtungen schliessen lässt. Somit kann das Morgenbeben als ziemlich gesichert gewertet werden.

Freising: $\quad$ in nocte ante diem et in die secundo

Petershausen: circa tertiam noctis vigiliam, et iterum in ipsa die undecima fere hora

St. Blasien: in galli cantu semel et ad nonam secundo

Zwettl: bis, unus post tertium galli cantum, alter die e odem hora quasi 10

Zwiefalten: $\quad$ bis in nocte et die

Schäftlarn: $\quad$ semel in nocte et semel in die

Drei dieser sechs Quellen ist die grosse Ausdehnung der beiden Erdbeben bereits bekannt, was erneut auf eine nicht unmittelbare Niederschrift weist. In den Notizen aus Freising und Schäftlarn sind jedoch keine Fremdinformationen erkennbar, so dass sie oder ihre Vorlagen möglicherweise als ereignisnah gewertet werden dürfen. Gleichzeitig wird deutlich, dass der Nachmittagsstoss häufiger und verbreiteter verzeichnet worden war als das Morgenbeben. Damit handelt es sich beim Morgenbeben mit grösserer 
Wahrscheinlichkeit um ein kleineres und eher lokales Ereignis. Zusätzlich fällt auf, dass die neun Quellen mit zwei Erdbebebenbeobachtungen nur in drei Fällen Schadenberichte enthalten, einmal deutlich und zweimal andeutungsweise abgegrenzt von lokalen Beobachtungen. Somit hat das Morgenbeben mit grosser Wahrscheinlichkeit keine Schäden verursacht, was von der folgenden Analyse der in diesen und weiteren Quellen vorhandenen angeblichen Schäden bestätigt wird.

\section{Unzulässige Bewertung als Schadenbeben}

So stellt sich noch die Frage, ob das auch in Mitteleuropa verspürte Nachmittagsbeben, das sogenannte Veronese-Beben, die erwähnten Schäden verursacht haben könnte. Doch eine vertiefte Analyse der Quellentexte muss auch die nicht zeitlich fixierten Schäden in Frage stellen. Umgekehrt ist die These von Hülsen (1993), dass es sich insgesamt um eine Quellenkontamination in Mitteleuropa handle, ähnlich wie es für das Erdbeben von 1348 nachweisbar sei, zu strikt, denn die lokalen Berichte sind sehr individuell formuliert, und stimmen in den Fakten für zwei Erdbeben überein. Vielmehr gilt es nun zu belegen, dass es sich um eine Vermischung von lokalem Verspüren eines kleineren Erdbebens am Morgen mit Fremdinformationen über Schäden in Italien am Nachmittag handelt.

\section{Analyse des Beschreibungsmusters}

In den meisten der 40 nordalpinen Erdbeben-Beschreibungen (Alexandre 1990, S. 147154) werden die allfälligen Schadensberichte deutlich Italien zugeordnet, was bereits gegen die wenigen ausdrücklich im Norden der Alpen lokalisierten Schäden spricht. Doch die Ursache der Fehlinterpretationen lässt sich mit einer Analyse des Beschreibungsmusters noch genauer darstellen:

- Jahr

- Tag und Monat

- Ungefähre Zeitangabe

- Begriff für das Ereignis (terrae motus)

- Anzahl der beobachteten Ereignisse

- Schadensbeschreibung

- Lokalisierung

1. Wenn keine Lokalisierungsabsicht erkennbar ist, lässt sich die Aussage nur bedingt auswerten.

2. Eigenbeobachtung: Wenn eine oder mehrere Lokalisierungen erkennbar sind, kann eine erstplazierte Quellenaussagen ohne Lokalisierung mit einer gewissen Wahrscheinlichkeit als Eigenbeobachtungen des annalenführenden Klosters gewertet werden, vor allem auch, wenn sie im Vergleich mit anderen Quellen sehr unterschiedlich formuliert worden sind.

3. Fremdinformationen: In den meisten Fällen werden erst nach den Eigenbeobachtungen allfällige Fremdbeobachtungen aufgeführt und mit einem geografischen Begriff wie „Italien“ oder „Lombardei“ klar abgegrenzt. Manchmal aber ist die Abgrenzung nur angedeutet mit „in der ganzen Welt“, „an gewissen Orten“, allenfalls auch mit einem Indefinitpronomen wie „überall“. 
Damit kommt der Interpretation des Übergangs von der lokalen Eigenbeobachtung zu den Fremdinformationen und der Uhrzeit zentrale Bedeutung zu, wenn man vermeiden will, dass italienische Schäden des Nachmittagbebens irrtümlich beim annalenführenden Kloster nördlich der Alpen lokalisiert wurden; wie die zwei folgenden Beispiele zeigen.

\section{Zwei Fehllokalisierungen von Schäden}

So meldet die Quelle aus dem Kloster Petershausen bei Konstanz erst nach der Lokalisierung „weit und breit auf dem Erdkreis“ Schäden, womit die erwähnten Einstürze von vielen Kirchen und Städten - man beachte den Plural - nicht auf Konstanz bezogen werden können, sondern meinen mit grösster Wahrscheinlichkeit Verona und Italien: „De terre motu. ... anno dominicae incarnationis 1117,3 . Non. lanuarii circa tertiam noctis vigiliam, [et iterum in ipsa die undecima fere hora,] terrae motus ingens factus est longe lateque per orbem, ita ut aecclesiae multae et quedam urbes ex immensa concussione subrueruntur. [Von einem Erdbeben. (...) Im Jahre des Herrn 1117, am 3. Januar, ungefähr im 3. Viertel der Nacht ${ }^{1}$ [und wiederum am selben Tag, ungefähr um die elfte Stunde], ereignete sich ein ausserordentliches Erdbeben weit und breit auf dem Erdkreis, so dass viele Kirchen und einige Städte durch das ungeheure Beben zum Einsturz gebracht wurden."] (Casus monasterii Petrishusensis, MGH SS 20 (1869), S. 661: 2.). Guidoboni (2005a) schätzt jedoch die Intensität im Kloster Petershausen bei Konstanz auf 7 .

Ähnlich verhält es sich mit einer Meldung aus dem 75 km von Konstanz entfernten Kloster St. Blasien im Schwarzwald: „Tercio Non. lanuar. terre motus fuit ubique tam validus, ut turres aliaque edificia plura subruerent, in galli cantu semel, et ad nonam secundo. [Am 3. Januar war überall ein derart starkes Erdbeben, dass mehrere Türme und andere Gebäude einstürzten, der erste Stoss beim Hahnenschrei, ${ }^{2}$ der zweite um die 9. Stunde (des hellen Tages).] “ Die eigenständige zeitliche Umschreibung in beiden Quellen lässt auf eine lokale Eigenbeobachtung schliessen, die Schadensberichte müssen aber auf Fremdinformationen beruhen, da sie „überall“ lokalisiert werden. Die in die oben erwähnte Petershausener Quelle hineininterpretierten angeblichen Zerstörungen im ziemlich nahen Konstanz werden hier nicht erwähnt, womit es sehr wahrscheinlich wird, dass es sich vielmehr um Fremdinformationen über Schäden in Italien handelt. Somit sind die Intensitäten in Alexandre (1990) und Guidoboni (2005a) für diese Orte auf 5 zu reduzieren.

\section{Angebliche Schäden in Augsburg und Umgebung und in Konstanz}

Zu den zwei Ausnahmen mit ausdrücklich in Süddeutschland lokalisierten Schäden gehört die Beschreibung in der Chronik von Bertholdus von Zwiefalten (Chronicon Monasterii Zwifaltensis, MGH SS 10, 1852, S. 96-124), wo über die angebliche Zerstörung der Haigirinburg/Heirenbuch bei Augsburg und einem zerstörten Turm in Augsburg selber mit auffallender Ausführlichkeit berichtet wird. Darauf basierend schätzt Guidoboni (2005a) die Intensität des Bebens auf 8-9 und lokalisiert es in Augsburg.

Mehrere Punkte lassen aber an der Faktizität des Berichtes von Bertholdus zweifeln. So zeigt die Form der Erzählung deutliche Züge eines bekannten Erdbebentopos, wie ihn

1 Tertia vigilia entspricht der Hora galli cantus, also ungefähr um 2 Uhr, Lokalzeit.

2 Hora galli cantus entspricht der Tertia vigilia, also ungefähr um 2 Uhr, Lokalzeit. 
beispielsweise schon Cassius Dio für das Erdbeben in Antiochia im Jahre $115 \mathrm{n}$. Chr. verwendete (Sonnabend, 1999, S. 51): eine stillende Mutter wird mit dem Säugling in einem Burgturm verschüttet, wobei die Mutter schwer verletzt wird, das (unschuldige) Kind aber unversehrt bleibt, während die übrigen Bewohner tot sind. Eine ähnliche Erzählung lässt sich auch für das Erdbeben von 1356 und den von einem Erdbeben ausgelösten Bergsturz von 1584 belegen. Daraus lässt sich schliessen, dass es Bertholdus mehr um die Darstellung und Interpretation des Phänomens Erdbeben am Beispiel von 1117 ging als um eine Beschreibung der süddeutschen Fakten. Im weiteren ist zu beachten, dass diese Chronik erst zwischen 1137 und 1139 verfasst wurde, der Anhang zwischen 1139-47, also in einer Distanz von mindestens 20 Jahren zu den Ereignissen, und damit die Darstellungsintention des Textes wahrscheinlich über das Verfassen eines Augenzeugenberichtes hinausgeht. Ausserdem ist das Original verschollen, nur eine Abschrift aus dem 17. Jahrhundert existiert. Im Weiteren soll eine rhetorische Floskel von den knappen Fakten ablenken: „Von diesem Erdbeben könnten wir vieles Erstaunliche, Unerhörte und Erschreckende erzählen, wenn es nicht nach der Anweisung des Hieronymus unser Vorsatz wäre, uns in allen Dingen der Kürze zu befleissigen.“

Ferner spricht auch die Erdbebenbeschreibung in der Chronik von Heimo von Bamberg, der vermutlich aus einem Augsburger Codex abgeschrieben hat (MGH SS 10, 1852, S. 8-11), gegen die Bertholdsche Schadensthese, denn dort werden überhaupt keine Schäden aufzählt. Zusätzlich erwähnt Berthold ausdrücklich, dass er sich zur Zeit des Bebens in Böhmen befunden habe. Ausserdem setzte er den Zeitpunkt des Bebens auf die Vesper an, ohne das Morgenbeben zu erwähnen. Eine parallele Quelle, die zeitgenössischen Annales maiores Zwifaltenses (MGH SS 10 (1852), S. 53-64), erwähnt das Ereignis ebenfalls, sie datiert richtig und impliziert mit der Erwähnung von zahlreichen Todesopfern entsprechende Schäden, deren Zuordnung ist aber weder zu Italien noch zu Süddeutschland philologisch eindeutig machbar.

Die Zwiefaltener Chronik von Bertholdus berichtet zusätzlich von einer angeblichen Veränderung der Sichtverhältnisse zwischen Meersburg und Konstanz („... die Glockentürme und andere ebenso hohe Gebäude, die in der Stadt Konstanz erbaut sind, welche vor diesem Erdbeben vom Schloss Meersburg aus nicht gesehen werden konnten, sind jetzt für jeden Beobachter sichtbar"); ${ }^{3}$ Guidoboni interpretiert diese Beschreibung als „landslide or raising of one place as compared with another“. Wenn man dies nicht als weiteren Erdbeben-Topos werten will (ähnlich antiken Vorstellungen vom Auftauchen und Verschwinden von Inseln [Masciadri, 2008]), so könnten damit am ehesten Veränderungen am kleinen Hügel bei Petershausen, der auf der Landzunge nordwestlich von Konstanz liegt, gemeint sein. Genau von dort stammt die bereits erwähnte zweite lokale Quelle (Casus monasterii Petrishusensis), sie aber erwähnt keinerlei Veränderung in der Landschaft. Damit müssen beide Schadensbeschreibungen der Zwiefaltener Quelle klar in Frage gestellt werden.

3 ,... cloclea cum aliis aedificiis aeque altis in Constantiensi civitate constituta, de castello Mercisburc dicto ante hunc terrae motum nunquam visa, modo cunctis cernentibus apparent universa." (Bertholdus von Zwiefalten (MGH SS 10, 1852, S. 112)). 


\section{Kleiner Schaden an baufälliger Bamberger Kirche}

Den zweiten Sonderfall der nordalpinen Schadensbeschreibungen stellt ein kleiner Schaden an der Michaelskirche in Bamberg dar, festgehalten in der Vita Ottonis (MGH SS 12, 1856, S. 822 ff), die jedoch erst um 1150 verfasst worden war, also mehr als 30 Jahre nach dem Ereignis. Diese Quelle fixiert den Schaden ausdrücklich auf die Vesperstunde, ohne ein Morgenbeben zu erwähnen. Der angebliche Erdbebenschaden wird bereits in diesem Bericht hauptsächlich auf den ausdrücklich erwähnten schlechten Zustand des Gebäudes zurückgeführt. Dabei handelt es sich um einen einzelnen grossen Stein, der vom obersten Punkt des Gewölbes heruntergefallen sei, während der Rest stehen blieb. Da zwei weitere Bamberger Quellen, nämlich die Annales S. Petri Babenbergensis (MGH SS 17, 1861, S. 636-637) und die Annales S. Michaelis Babenbergensis (MGH SS 5, 1844, S. 9-10), nur von einem verspürten Beben sprechen, beziehungsweise von Schäden in Italien, ist der fallende Stein bei Ebbo mit grosser Wahrscheinlichkeit als retrospektive Interpretation eines bestehenden Bauschadens zu verstehen.

Um künftig das tatsächliche Schüttergebiet des Nachmittagsbebens abzuschätzen zu können, müssen die mitteleuropäischen Quellen in derselben Weise in Eigenbeobachtungen und Fremdinformationen aufgeteilt werden, vgl. die Notizen zum Veronese-Beben.

\section{Schlussfolgerung}

Am 3. Januar 1117 wurden in Süddeutschland zwei Erdstösse verspürt. Aber die angeblichen Schäden im süddeutschen Gebiet sind für beide Erdbeben mit grösster Wahrscheinlichkeit als Fehlinterpretationen der Quellen zu werten. Ausserdem müsste ein Beben der Stärke 8-9 in Mitteleuropa zu dieser Zeit bereits deutlichere dokumentarische Spuren hinterlassen haben, ähnlich wie andere umweltgeschichtliche Extremereignisse, z. B. der Extremwinter 1076/77 (Schwarz-Zanetti, 1992).

Makroseismische Parameter für das süddeutsche Morgenbeben vom 3. Januar 1117

\begin{tabular}{|l|l|l|l|l|l|l|}
\hline Ortschaft & Länge & Breite & $I_{\min }$ & $I_{\max }$ & $I_{\text {w }}$ & Datenqualität \\
\hline Augsburg, D & 48.37 & 10.9 & 4 & 5 & verspürt & sehr schwach \\
\hline Freising, D & 48.4 & 11.75 & & & verspürt & sehr schwach \\
\hline St. Blasien, D & 47.76 & 8.13 & 4 & 5 & verspürt & sehr schwach \\
\hline Zwettl, A & 48.61 & 15.17 & & & verspürt & sehr schwach \\
\hline Zwiefalten, D & 48.23 & 9.46 & 4 & 5 & verspürt sehr schwach \\
\hline Odernheim am Glan, D & 49.76 & 7.72 & & & verspürt sehr schwach \\
\hline Salzburg, A & 47.83 & 13.05 & & & verspürt sehr schwach \\
\hline Konstanz, D & 47.67 & 9.18 & 5 & 5 & verspürt & sehr schwach \\
\hline Schäftlarn, D & 47.98 & 11.46 & 5 & 5 & verspürt & sehr schwach \\
\hline
\end{tabular}

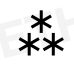




\begin{tabular}{|l|l|l}
\hline Datum & Zeit & Ort \\
\hline 1117, 3. Januar & 13 Uhr & Veronese, I
\end{tabular}

\section{Bewertung des Ereignisses}

Sicheres Ereignis.

\section{Parameter nach ECOS}

Ix: 9

\section{Publiziert u. a. in:}

- Alexandre (1990)

- Hülsen (1993)

- Guidoboni (2005a, b)

- DOM4.1

Das sogenannte Veronese-Beben vom Nachmittag des 3. Januar 1117 wurde auch in Mitteleuropa verspürt und zahlreich festgehalten, so erwähnen auch die Annales Einsidlenses (MGH SS 3, 1839, S. 137ff.) ein Erdbeben an diesem Tag, ohne jedoch die Stunde zu spezifizieren. Der Schütterradius erstreckt sich gemäss Alexandre (1990) bis nach Hamburg, was aus seismologischen Gründen knapp möglich ist. Ebenso gut kann es sich in grösserer Entfernung von Verona um eine Vermischung mit Fremdinformationen handeln (Hülsen, 1993). Das mittelalterliche Informationsnetz sollte nicht unterschätzt werden, wie das Erdbeben von Syrien im Jahre 1170 zeigt, das in acht zeitgenössischen mitteleuropäischen Quellen sieben Mal richtig datiert und lokalisiert wird.

$$
\stackrel{*}{* *}
$$

\begin{tabular}{|c|c|c|}
\hline $\begin{array}{l}\text { Datum } \\
\text { - } \quad 1127,24 . \text { März } \\
\text { - } \quad 1127,13 . \text { April }\end{array}$ & $\begin{array}{l}\text { Zeit (UTC) } \\
-\quad \text { Gegen Mitter- } \\
\text { nacht } \\
-\quad\end{array}$ & $\begin{array}{l}\text { Ort } \\
\text { - } \quad \text { Nicht lokalisierbar } \\
\quad \text { (St. Blasien, D) } \\
\text { - } \quad \text { Nicht lokalisierbar } \\
\quad \text { (Regensburg, D) }\end{array}$ \\
\hline
\end{tabular}

\section{Bewertung der Ereignisse}

Unsichere Ereignisse.

\section{Parameter nach ECOS}

- 24. März-lx: verspürt

- 13. April-lx: verspürt 


\section{Überlieferung u. a. in:}

- Sieberg (1940), S. 28: „1127. März 25, gegen Mitternacht. Erdbeben im Schwarzwald wahrscheinlich auch in St. Blasien." (Lersch, GFZ.)

- Alexandre (1990)

Keine Hinweise in:

- Bertrand (1756)

- Volger (1857)

- Giessberger (1922)

\section{Quelle}

24. (25.) März 1127:

Annales S. Blasii et Engelbergenses (MGH SS 17, 1861, S. 278): „1127. Factus est terremotus 8. Kal. April. media nocte.“ [Am 25. März ereignete sich um Mitternacht ein Erdbeben.]

\section{April 1127:}

- Annales Ratisponenses (MGH SS 17, 1861, S. 585)

- $\quad$ Annales Gotwicenses (MGH, SS 9, 1851, S. 601-604)

- Annales et Notae Schaeftlarienses (MGH SS 17, 1861, S. 334-342)

\section{Interpretation}

Die Annales S. Blasii et Engelbergenses, zu diesem Zeitpunkt in St. Blasien im Schwarzwald, $15 \mathrm{~km}$ nördlich der Schweizer Grenze, geführt, sind für seismologische Vorgänge im 12. Jahrhundert eine sehr aufmerksame Quelle, was die Meldung relativ vertrauenswürdig macht.

Wegen der Zeitangabe „Mitternacht“ muss aber auf den 24. März 1127 zurückdatiert werden.

Es ist aber unklar, ob die drei Hinweise aus den Annales Ratisponenses (Regensburg), Annales Gotwicenses (Göttweig, A) und Annales et Notae Schaeftlarienses (Schäftlarn, D), datiert auf den 13. April 1127, mit dem März-Ereignis zusammenhängen, denn es fehlen Hinweise auf das vorhergehende Beben. Ohne eine zeitgenössische Bestätigung für den 24. März 1127 muss auch dieses Ereignis vorläufig als „unsicher“ und wegen fehlender Angaben zur Intensität mit „verspürt“ gewertet werden.

\section{Makroseismische Parameter}

24. März 1127

\begin{tabular}{|l|l|l|l|l|l|l|}
\hline Ortschaft & Länge & Breite & $I_{\min }$ & $I_{\max }$ & $I_{w}$ & Datenqualität \\
\hline St. Blasien, D & 47.76 & 8.13 & & & verspürt & sehr schwach \\
\hline
\end{tabular}


13. April 1127

\begin{tabular}{|l|l|l|l|l|l|l|}
\hline Ortschaft & Länge & Breite & $I_{\min }$ & $I_{\max }$ & $I_{W}$ & Datenqualität \\
\hline Schäftlarn, Isartal, D & 48.67 & 11.47 & 5 & & & sehr schwach \\
\hline Regensburg, D & 49.02 & 12.09 & 5 & & & sehr schwach \\
\hline Furth bei Göttweig, A & 48.22 & 15.36 & 5 & & sehr schwach \\
\hline
\end{tabular}

\begin{tabular}{l|l|l}
\hline Datum & Zeit & Ort \\
\hline 1127, 25. März & 23 Uhr & Waldshut-Tiengen, D
\end{tabular}

\section{Bewertung des Ereignisses}

Kein Erdbeben: Datierungsfehler (und Lokalisierungsfehler).

\section{Korrektur}

24. März 1127

\section{Überlieferung}

- Sieberg (1940): S. 28: „1127, März 25, gegen Mitternacht. Erdbeben im Schwarzwald wahrscheinlich auch in St. Blasien. " (Lersch, GFZ.)

- Alexandre (1990): 25. März, St. Blasien im Schwarzwald. Note: Il doit s'agir de la nuit du 24 au 25/3.

- MECOS-99

Keine Hinweise in:

- $\quad$ Bertrand (1756)

- Volger (1857)

- Giessberger (1922)

\section{Quelle}

Annales S. Blasii et Engelbergenses (1861), MGH SS 17, S. 278: 1127. Factus est terremotus 8. Kal. April. media nocte. [Am 25. März ereignete sich um Mitternacht ein Erdbeben.]

\section{Interpretation}

Da der vorliegende Katalog bei genauen Uhrzeiten von der Lokalzeit in UTC umrechnet, muss dieses Erdbeben auf den 24. März zurückdatiert werden.

\section{Bemerkungen}

Vgl. das Erdbeben vom 24. März 1127 in St. Blasien, D.

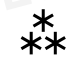




\section{\begin{tabular}{l|ll} 
Datum & Zeit & Ort \\
1128 & - & Reinach-Basel
\end{tabular}}

\section{Bewertung des Ereignisses}

Kein Erdbeben: Datierungs- und Lokalisierungsfehler.

\section{Korrektur}

29. Juni 1128, St. Blasien-Ellwangen, D

\section{Überlieferung u. a. in:}

- Bertrand (1756)

- Volger (1857)

- Sieberg (1940)

- MECOS-99

\section{Quelle}

- Annales Elwangenses (MGH SS 10, 1852, S. 34-51)

- $\quad$ Annales S. Blasii et Engelbergenses (MGH SS 17, 1861, S. 275-282)

\section{Interpretation}

Die Annales Elwangenses erwähnen ein Erdbeben ohne Tagesdatum: „1128. ... et terrae motus magnus factus est. “ [(1128) ... und es ereignete sich ein grosses Erdbeben.]

Möglicherweise haben sich die Kompilatoren des angeblichen Reinach-Basler Bebens auf diese Ellwanger Quelle gestützt, deren Datierung aber mit einer gewissen Wahrscheinlichkeit im Sinne der Annalen von St. Blasien präzisiert werden darf, die den 29. Juni 1128 nennen.

\section{Bemerkungen}

Vgl. das Erdbeben vom 29. Juni 1128 in St. Blasien-Ellwangen, D.

\section{***}

\begin{tabular}{|l|l|l} 
Datum & Zeit & Ort \\
11128 (29. Juni) & - & Nicht lokalisierbar \\
& & (St. Blasien-Ellwangen, D)
\end{tabular}

\section{Bewertung des Ereignisses}

Unsicheres Ereignis.

\section{Parameter nach ECOS}

Ix: verspürt

\section{Überlieferung u. a. in:}

- Lycosthenes (1557)

- Bertrand (1756) 
- Guéneau de Montbelliard (1761)

- Volger (1857)

- Langenbeck (1892)

- Stichler (1906)

- Giessberger (1922)

- Sieberg (1940)

- Alexandre (1990)

- MECOS-99

\section{Quellen}

- Annales S. Blasii et Engelbergenses (MGH SS 17, 1861, S. 275-282): „1128. Iterum 3. Kal. lul. factus terre motus nocte." [Am 29. Juni 1128 ereignete sich in der Nacht wiederum ein Erdbeben.]

- Annales Elwangenses (MGH SS 10, 1852, S. 34-51) ohne Tagesdatum: „1128. ... et terrae motus magnus factus est.“ [(1128) ... es ereignete sich ein grosses Erdbeben.]

\section{Interpretation}

Seit der Arbeit von Elie Bertrand (1756) findet sich in vielen Kompilationen die Meldung von einem (oder mehreren) Beben in der Schweiz im Jahre 1128, das angeblich 40 Tage dauerte und eine grosse Zahl von Häusern erschütterte: „En mille cent vingt-huit, on sentit en Suisse, \& ailleurs des Tremblemens, qui durèrent quarante jours; on remarqua des retours de secousses par intervalles; grand nombre de maisons furent ébranlées." Bertrand gibt keine Tagesdatierung an.

Zwei Besonderheiten fallen bei Betrand auf; zum einen deutet die ausführliche Beschreibung nicht auf eine hochmittelalterliche Vorlage hin, sondern zumindest auf eine jüngere Interpretation, zum anderen enthält die Zahl 40 auch eine biblische Komponente, die auch bei der Beschreibung des Bebens von 1117 auftaucht. Diese zwei Merkmale lassen sich aber bei den zeitgenössischen Berichten aus St. Blasien für den 29. Juni 1128 und aus Ellwangen, ohne Tagesdatierung, nicht finden (Alexandre 1990). Betrachtet man die Ausdehnung von Oberitalien bis ins Elsass in Sieberg (1940), so weist sie auch auf eine Verwechslung mit 1117 hin. Ausserdem enthält Lycosthenes (1557) noch einen Hinweis auf Tyros im Libanon 1128.

Mit zwei zeitgenössischen Quellen ist dieses Beben ohne Schäden in Süddeutschland im Jahr 1128 historisch als „unsicher“ zu werten.

\section{Makroseismische Parameter}

\begin{tabular}{|l|l|l|l|l|l|l|}
\hline Ortschaft & Länge & Breite & $I_{\min }$ & $I_{\max }$ & $I_{w}$ & Datenqualität \\
\hline St. Blasien, D & 47.76 & 8.13 & & & verspürt & sehr schwach \\
\hline Ellwangen (Jagst), D & 48.96 & 10.13 & & & verspürt & sehr schwach \\
\hline
\end{tabular}




\section{Datum \\ Zeit (UTC) \\ Ort \\ 1134, 12. Januar \\ Vor Mitternacht \\ Nicht lokalisierbar (St. Blasien, D)}

\section{Bewertung des Ereignisses}

Unsicheres Ereignis.

\section{Parameter nach ECOS}

Ix: verspürt

\section{Überlieferung u. a. in:}

- Sieberg (1940)

- Rothé und Schneider (1968)

- Alexandre (1990)

- MECOS-99

\section{Quelle}

Annales S. Blasii et Engelbergenses (MGH SS 17, 1861, S. 278): „1134. Pridie Id. Ian.

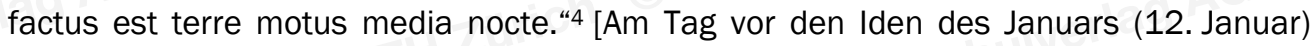
ereignete sich ein Erdbeben um Mitternacht.]

\section{Interpretation}

Zum Beben von 1134 ist nur diese eine Quelle aus St. Blasien im Schwarzwald bekannt. Bei diesen Annalen handelt es sich jedoch um zeitgenössische und für seismologische Ereignisse sehr aufmerksame Aufzeichungen, sodass diese Einzelbeobachtung als „unsicher" gewertet werden darf. Eine Beschreibung der Intensität fehlt.

\section{Makroseismische Parameter}

\begin{tabular}{|l|l|l|l|l|l|l|}
\hline Ortschaft & Länge & Breite & $I_{\min }$ & $I_{\max }$ & $I_{w}$ & Datenqualität \\
\hline St. Blasien & 47.76 & 8.13 & & & verspürt & sehr schwach \\
\hline
\end{tabular}

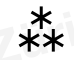

\begin{tabular}{l|l|l} 
Datum & Zeit & Ort \\
\hline 1134, 12. Januar & - & Waldshut-Tiengen, D
\end{tabular}

\section{Bewertung des Ereignisse}

Kein Erdbeben: Lokalisierungsfehler.

\section{Korrektur}

12. Januar 1134 in St. Blasien

4 Nacht vom 11. auf den 12. Januar. 


\section{Überlieferung}

- Sieberg (1940), stützt sich auf das Manuskript Lersch, GFP.

- Gils, Van \& Leydecker (1991)

- MECOS-99

\section{Interpretation}

Die Herkunft der Lokalisierung „Waldshut-Tiengen“ im MECOS-99 (Ühlingen, Kreis Landshut), bzw. in Baden-Württemberg in Van Gils \& Leydecker (1991) konnte nicht eruiert werden, mit grosser Wahrscheinlichkeit handelt es sich aber um die Einzelquelle aus St. Blasien.

\section{Bemerkungen}

Vgl. das Erdbeben vom 12. Januar 1134 in St. Blasien, D.

\begin{tabular}{|c|c|c|}
\hline $\begin{array}{l}\text { Datum } \\
1152,-\end{array}$ & Zeit & $\begin{array}{l}\text { Ort } \\
\text { Neuchâtel }\end{array}$ \\
\hline
\end{tabular}

\section{Bewertung des Ereignisses}

Kein Erdbeben: Lokalisierungsfehler.

\section{Korrektur}

Italien (eventuell Monte Cassino)

Überlieferung u. a. in:

- Rasch (1591)

- MECOS-99

Mögliche Quellen (Alexandre 1990):

- Annales Casinenses (1866)

- Continuatio quae dicitur praemonstratensis (1844)

\section{Interpretation}

Der MECOS-99-Eintrag beruht auf der Erdbeben-Chronik von Rasch (1591): „1152 Erdbiden in Welschland“. Damit bezieht sich Rasch aber mit grosser Wahrscheinlichkeit auf ein Erdbeben in Italien, wie es beispielsweise in den Annales Casinenses (Monte Cassino, Italien; MGH SS 19, 1866, S. 302-320) für das Jahr 1152 verzeichnet ist. Die „Continuatio quae dicitur praemonstratensis“ (Laon, F) erwähnen ebenfalls ein Erdbeben in Italien in diesem Jahr; vgl. Alexandre (1990). Im MECOS-99 wurde diese Notiz aber aufgrund eines philologischen Irrtums bei der Übersetzung des Begriffes "Welschland“ im schweizerischen Neuenburg lokalisiert. Doch der Begriff "Welschland“ bezeichnete im 
16. Jahrhundert nicht die Romandie, wie es seit dem 19. Jahrhundert in der Schweiz üblich ist, sondern Italien.

Auszug aus dem Grimmschen Wörterbuch (1854-1922, Bd. 27 s.v.): „Wälsch“ (col. 1328): ... dasz es entweder überhaupt den gegensatz zu deutsch bildet oder, auf eine der romanischen nationen gehend, für „,italienisch“ oder „französisch“, seltener „spanisch“ oder „rätoromanisch“ steht. vom 16. jahrhundert an tritt die bedeutung „italienisch“ in den vordergrund (die erst gegen 1800 zu veralten beginnt) ... . Und Bd. 27 s.v. „Wälschland“ (col. 1356): die gewöhnliche bedeutung ist die von „Italien“, die auch jetzt noch, namentlich in der poetischen sprache, üblich ist.

\section{Bemerkungen}

Vgl. Alexandre (1990).

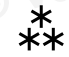

\begin{tabular}{|l|l|l}
\hline Datum & Zeit (UTC) & Ort \\
\hline $1155,18$. Januar & $23 \mathrm{Uhr}$ & Laon, F (?)
\end{tabular}

\section{Bewertung des Ereignisses}

Sicheres Ereignis.

\section{Parameter nach ECOS}

Ix: verspürt

Überlieferung u. a. in:

- Sieberg (1940)

- Alexandre (1990)

- MECOS-99

- SisFrance (6. April 2009)

\section{Quellen}

Gemäss Alexandre (1990, S. 159-160) berichten acht zeitgenössische Quellen über ein Erdbeben im Burgund im Januar 1155, und zwar aus Laon(?), Sens, Nevers, Mont-St.Michel in den Vogesen, Dijon, Cluny, Bonnevaux und Engelberg, $\mathrm{CH}$, (die letzte Quelle lokalisiert er irrtümlich in St. Blasien, D). Teilweise werden Schäden aufgeführt.

Neue Lokalisierung: Annales S. Blasii et Engelbergenses (MGH SS 17, 1861, S. 279): „1155. 15. Kal. Febr. factus est terre motus 6. hora noctis.“ [Am 18. Januar 1155 ereignete sich in der 6 . Stunde der Nacht ${ }^{5}$ ein Erdbeben.]

5 In der 6. Stunde der Dunkelheit, also um Mitternacht, bzw. 23 Uhr (UTC). 


\section{Interpretation}

Dieses Erdbeben konnte im Rahmen der Revision des Schweizerischen Erdbebenkatalogs nicht genauer untersucht werden, obwohl die Schweiz mitbetroffen ist. Die Karte von Alexandre (1990, S. 238) zeigt Cluny als Epizentrum, die Intensität wird dort mit 7-8 umschrieben. SisFrance (6. April 2009) gibt hingegen keine Intensitätsangabe und lokalisiert das Beben im Plateaux jurassiens (Lons-le-Saulnier?) - Franche-Comté.

Zwar scheinen die Berichte in der Datierung und den meisten Einzellokalisierungen zuverlässig zu sein, widersprüchlich sind hingegen die Intensitätsbeschreibungen. Der Aussage aus dem Chronicon des Robert du Mont aus den Vogesen, die toposartig von einer von den Abgründen verschlungenen Burg nahe von Cluny spricht, widersprechen die Iokalen Annales Cluniacenses, die nur von Erschütterungen sprechen. Damit ist grundsätzlich die Intensität von 7-8 auf „verspürt“ zu reduzieren. ${ }^{6}$ Die zweite Schadensmeldung stammt aus Laon und weist ausdrücklich darauf hin, dass es sich nicht um eine Eigenbeobachtung handelt. Auch die Chronica universalis Senonensis spricht nur von Gefahr an wenigen Orten. Die übrigen fünf Quellen erwähnen keine Schäden (nach Alexandre, 1990).

Alexander lokalisiert den Bericht aus den Annalen von St. Blasien und Engelberg (MGH SS 17, 1861, S. 275-282) noch in St. Blasien statt in Engelberg. Doch Schmales Neuausgabe von Wattenbach (1967-71) und das On-line-Verzeichnis "Geschichtsquellen des deutschen Mittelalters“ postulieren, dass diese bereits ab 1147 in Engelberg geführt worden sind.

\section{Makroseismische Parameter (nur für Engelberg, OW und Cluny, F)}

\begin{tabular}{|l|l|l|l|l|l|l|}
\hline Ortschaft & Länge & Breite & $I_{\min }$ & $I_{\max }$ & $I_{\text {w }}$ & Datenqualität \\
\hline Engelberg & 46.82 & 8.4 & & & verspürt & sehr schwach \\
\hline Cluny, F & 46,43 & 4.67 & & & verspürt & schwach \\
\hline
\end{tabular}

\section{Bemerkungen}

Vgl. die Sitepunkte im französischen Katalog SisFrance.

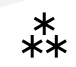
Datum
1155, 18. Januar
Zeit (UTC)
Ort
$17 \mathrm{Uhr}$
Waldshut-Tiengen, D

\section{Bewertung des Ereignisses}

Kein Erdbeben: Lokalisierungsfehler.

6 Vgl. die Einleitung und das süddeutsche Erdbeben vom 3. Januar 1117, wo ebenfalls toposartige Beschreibungen die Darstellung des Erdbebens verfälschten. 


\section{Korrektur}

18. Januar 1155, Laon, F

\section{Überlieferung}

- Sieberg (1940)

- Gils, Van \& Leydecker (1991)

- MECOS-99

\section{Interpretation}

Die Herkunft der Lokalisierung „Waldshut-Tiengen“ im MECOS-99 konnte nicht mehr eruiert werden. Van Gils \& Leydecker (1991) lokalisieren erneut ohne Begründung in Ühlingen, D. Sieberg (1940) dagegen vermutet das Erdbeben im Schwarzwald oder in St. Blasien und datiert es irrtümlich auf den 15. Februar vor, gegen 18 Uhr (17 Uhr UTC), aufgrund einer falschen Umrechnung der Angaben im römischen Kalenderstil.

\section{Bemerkungen}

Vgl. das Erdbeben vom 18. Januar 1155 in Laon, F, im SisFrance-Katalog und im ECOS-09.

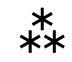

\begin{tabular}{l|l|l} 
Datum & Zeit & Ort \\
1158-1175 & - & Nicht lokalisierbar \\
(Engelberg, OW)
\end{tabular}

\section{Bewertung der Ereignisse}

Unsichere Ereignisse.

\section{Parameter nach ECOS}

\begin{tabular}{|l|l|l|l|l|}
\hline \multicolumn{2}{|c|}{ Datum } & Zeit (UTC) & Intensität \\
\hline Ix: & 1158 & 21. Jan. & $23 \mathrm{Uhr}(?)$ & verspürt \\
\hline 1161 & 22. Feb. & $16 \mathrm{Uhr}(?)$ & verspürt \\
\hline & 16. Juli & $16 \mathrm{Uhr}(?)$ & verspürt \\
\hline 1162 & 25. Feb. & $23 \mathrm{Uhr}$ & verspürt \\
\hline & 2. Dez. & $1 \mathrm{Uhr}$ & verspürt \\
\hline & 1170 & 1. April & $17 \mathrm{Uhr}(?)$ & verspürt \\
\hline & & $23 \mathrm{Uhr}(?)$ & verspürt \\
\hline & 1175 & 30. April & $19 \mathrm{Uhr}(?)$ & verspürt \\
\hline
\end{tabular}




\section{Überlieferung}

- Alexandre (1990)

\section{Quelle}

Die Annales S. Blasii et Engelbergenses (MGH SS 17, 1861, S. 275-282) wurden gemäss Wattenbach / Schmale (1967-71) und „Geschichtsquellen des deutschen Mittelalters“ (http://www.repfont.badw.de/A.pdf) ab 1147 in Engelberg geführt.

- 1158: 12. Kal. Febr. factus est terre motus 7. hora noctis. [Am 21. Januar 1158 ereignete sich in der 7. Stunde der $\mathrm{Nacht}^{7}$ ein Erdbeben.]

- 1161: 8 Kal. Mart. factus est terre motus ultima hora diei. Ipso etiam anno factus est alius terre motus 17. Kal. Aug. hora quasi vespertina. [Am 22. Februar $1161 \mathrm{er}-$ eignete sich in der letzten Stunde des Tages ${ }^{8}$ ein Erdbeben. Auch war im selben Jahr am 16. Juli etwa um die Vesperstunde ${ }^{9}$ ein anderes Erdbeben.]

- 1162: 5. Kal. Mart. factus est terrae motus gemina concussione media fere nocte. (...) Item eodem anno terrae motus ingens 4. Non. Dec. circa pullorum cantum. [Am 25. Februar 1162 ereignete sich ein Erdbeben ungefähr um Mitternacht mit doppeltem Stoss. Im selben Jahr war ein sehr starkes Erdbeben am 2. Dezember, ungefähr zur Zeit des Hahnenschreis. $]^{10}$

- 1170. Kal. Aprilis feria 4. ante cenam Domini factus est terre motus magnus penultima diei hora. ${ }^{11}$ Alius circa mediam noctem contigit. [Am 1. April 1170, Mittwoch, vor dem Abendmahl ereignete sich ein grosses Erdbeben in der zweitletzten Stunde des Tages. Ein anderes ereignete sich ungefähr um Mitternacht.]

- 1175. Pridie Kal. Maii terre motus factus est prima hora noctis. [Am Vorabend des ersten Mai (30. April) ereignete sich in der ersten Stunde der Nacht ein Erdbeben.] ${ }^{12}$

\section{Interpretation}

Gemäss den für seismische Vorgänge aufmerksamen zeitgenössischen Annalen S. Blasii et Engelbergenses wurden in der Zeit zwischen 1158 und 1175 acht Erdbeben in Engelberg, OW, verspürt, die sich jedoch noch nicht durch andere Beobachtungen bestätigen lassen. Indizien für allfällige Schäden sind nicht aufgeführt.

\footnotetext{
7 In der 7. Stunde der Nacht, also um Mitternacht, Lokalzeit.

8 Um 17 Uhr, Lokalzeit.

9 Um 17 Uhr, Lokalzeit.

10 Je nach Definition wird „Tertia vigilia“ oder „Hora galli cantus“, mit 2 Uhr morgens (1 UTC) umgerechnet, oder gemäss Dohrn (1996, S. 19) handelt es sich um die Zeit kurz vor der Dämmerung, also 7 Uhr (UTC), was jedoch vermutlich auf einem Fehlschluss in Niermeyer (1954-76) basiert.

11 Ungefähr um 18 Uhr abends, Lokalzeit.

12 Nacht vom 30. April auf den 1. Mai, ungefähr 19 Uhr, UTC.
} 


\section{Makroseismische Parameter}

21. Januar 1158:

\begin{tabular}{|l|l|l|l|l|l|l|}
\hline Ortschaft & Länge & Breite & $I_{\min }$ & $I_{\max }$ & $I_{\text {w }}$ & Datenqualität \\
\hline Engelberg & 46.82 & 8.4 & & & verspürt & sehr schwach \\
\hline
\end{tabular}

22. Februar 1161:

\begin{tabular}{|l|l|l|l|l|l|l|}
\hline Ortschaft & Länge & Breite & $I_{\min }$ & $I_{\max }$ & $I_{\text {w }}$ & Datenqualität \\
\hline Engelberg & 46.82 & 8.4 & & & verspürt & sehr schwach \\
\hline
\end{tabular}

16. Juli 1161:

\begin{tabular}{|l|l|l|l|l|l|l|}
\hline Ortschaft & Länge & Breite & $I_{\min }$ & $I_{\max }$ & $I_{w}$ & Datenqualität \\
\hline Engelberg & 46.82 & 8.4 & & & verspürt & sehr schwach \\
\hline
\end{tabular}

25. Februar 1162:

\begin{tabular}{|l|l|l|l|l|l|l|}
\hline Ortschaft & Länge & Breite & $I_{\min }$ & $I_{\max }$ & $I_{\text {w }}$ & Datenqualität \\
\hline Engelberg & 46.82 & 8.4 & & & verspürt & sehr schwach \\
\hline
\end{tabular}

2. Dezember 1162:

\begin{tabular}{|l|l|l|l|l|l|l|}
\hline Ortschaft & Länge & Breite & $I_{\min }$ & $I_{\max }$ & $I_{\text {w }}$ & Datenqualität \\
\hline Engelberg & 46.82 & 8.4 & & & verspürt & sehr schwach \\
\hline
\end{tabular}

1. April 1170, 17 und 23 Uhr:

\begin{tabular}{|l|l|l|l|l|l|l|}
\hline Ortschaft & Länge & Breite & $I_{\min }$ & $I_{\max }$ & $I_{w}$ & Datenqualität \\
\hline Engelberg & 46.82 & 8.4 & & & verspürt & sehr schwach \\
\hline
\end{tabular}

30. April 1175:

\begin{tabular}{|l|l|l|l|l|l|l|}
\hline Ortschaft & Länge & Breite & $I_{\min }$ & $I_{\max }$ & $I_{w}$ & Datenqualität \\
\hline Engelberg & 46.82 & 8.4 & & & verspürt & sehr schwach \\
\hline
\end{tabular}




\begin{tabular}{|l|l|l|}
\hline Datum & Zeit & Ort \\
\hline 1161, 16. Januar & - & Konstanz, D \\
\hline
\end{tabular}

\section{Bewertung des Ereignisses}

Kein Erdbeben: Lokalisierungsfehler (und Datierungsfehler).

\section{Korrektur}

1161, 16. Juli, um 17 Uhr (bzw. 16 Uhr UTC), in Engelberg

\section{Überlieferung u. a. in:}

- Lycosthenes (1557)

- Giessberger (1922)

- Sieberg (1940)

- Alexandre (1990)

- MECOS-99

Wahrscheinliche Quelle:

Annales S. Blasii et Engelbergenses (MGH SS 17, 1861, S. 275-282), ab 1147 in Engelberg geführt.

\section{Interpretation}

Es werden für die Jahre 1160 und 1161 mehrere Erdbeben-Daten genannt. So hat zum Beispiel Lycosthenes (1557) ein Erdbeben in Cotentin, Normandie, vom 1. Januar 1160 irrtümlich auf den 1. Januar 1161 umdatiert. Sieberg (1940) schreibt dann von Erdbeben in der Konstanzer Gegend und im Schwarzwald am 16. Januar und 22. Februar 1161, was eine Fehlübersetzung für "Cotentin“ (Constance) sein dürfte. Es gibt zwei zeitgenössische Beobachtungen in den Annales S. Blasii et Engelbergenses, die Teil dieser Vermischung sein dürften: 22. Februar und 16. Juli, vgl. Alexandre (1990). Es werden keine Schäden erwähnt.

\section{Bemerkungen}

Vgl. 1161 in der Tabelle der Engelberger Ereignisse 1158-1175.

\section{**}

\begin{tabular}{l|l|l} 
Datum & Zeit & Ort \\
1170 & - & Lugano
\end{tabular}

\section{Bewertung des Ereignisses}

Kein Erdbeben.

\section{Korrektur}




\section{Überlieferung}

- Gils, Van \& Leydecker (1991)

- MECOS-99

Nicht vorhanden in:

- Baratta (1901)

- DOM4.1

\section{Interpretation}

Die Lugano-Meldung stammt aus der Kompilation von Van Gils \& Leydecker, die keine Quellen angibt, und wurde vom MECOS-99 übernommen. Bertrand (1756) erwähnt ein grosses Schadenbeben in Sizilien, das aber möglicherweise eine Verwechslung mit dem Ereignis am 4. Februar 1169 ist (Alexandre 1990, S. 162).

Da keine Augenzeugenberichte oder weitere Kompilationen zu Lugano gefunden werden konnten, handelt es sich mit grosser Wahrscheinlichkeit um einen Irrtum. Es kann auch kein Korrekturvorschlag gemacht werden.

\section{***}

Datum

1170, 1. April
Zeit

17 und 23 Uhr
Ort

St. Blasien, D

\section{Bewertung des Ereignisses}

Keine Erdbeben: Lokalisierungsfehler

\section{Korrektur}

Nicht lokalisierbar (Engelberg).

\section{Überlieferung}

- Alexandre (1990)

\section{Interpretation}

Die Annales S. Blasii et Engelbergenses (MGH SS 17, 1861) wurden gemäss dem Editoren Pertz, Wattenbach-Schmale (1967-71) und dem Online-Register „Geschichtsquellen des deutschen Mittelalters“ ab 1147 in Engelberg geführt.

\section{Bemerkungen}

Vgl. im ECOS-09 das Erdbeben vom 1. April 1170 in Engelberg.

$$
\text { *** }
$$




\begin{tabular}{|l|l|l}
\hline Datum & Zeit & Ort \\
$1170,29$. Juni & - & - Bad Säckingen, D \\
& & - Steiermark, A
\end{tabular}

\section{Bewertung des Ereignisses}

Kein Erdbeben: Lokalisierungsfehler.

\section{Korrektur}

Syrien

\section{Überlieferung u. a. in:}

- Annales Admontenses (MGH SS 9, 1851, S. 570-593), zeitgenössische Quelle mit Verwechslung der Lokalisation

- Bertrand (1756)

- Volger (1857)

- Hoernes (1902)

- Giessberger (1922)

- Montandon (1942-43)

- MECOS-99

- Von Alexandre (1990) in der Falschbebenliste aufgeführt.

\section{Interpretation}

Acht zeitgenössische Meldungen aus Mitteleuropa bei Alexandre (1990) belegen, dass Nachrichten über das grosse Erdbeben in Syrien vom 29. Juni 1170 bald auch in Mitteleuropa umliefen. In den steirischen Annales Admontenses (Admont, A) geschah die Verwechslung von Syrien mit der Steiermark: Sijria wurde zu Stiria. Danach verbreitete sich das angeblich steirische Beben im Laufe der Zeit über Mitteleuropa und so auch in Schweizer Kompilationen, wie diejenigen von Bertrand (1756), Volger (1857) oder Montandon (1942-43).

\section{Bemerkungen}

In Alexandre (1990, S. 163-164) sind die europäischen Quellen aufgeführt, die das syrische Erdbeben erwähnen und meist richtig lokalisieren.

\section{**}

Datum

1180,1 . August
Zeit

3 Uhr
Ort

Stans, NW

\section{Bewertung des Ereignisses}

Kein Erdbeben: Datierungsfehler (und Lokalisierungsfehler). 


\section{Korrektur}

1179, 1. August, in Mitteleuropa (Brauweiler, D)

\section{Überlieferung}

Der älteste Nachweis für ein angebliches Beben in der Schweiz am 1. August 1180 findet sich in Bertrand (1756); auf inn stützen sich:

- $\quad$ Hoff (1840-1841)

- Perrey Rhin (1845)

- $\quad$ Volger (1857)

- Montandon (1942-43)

- Gils, Van \& Leydecker (1991)

- MECOS-99

\section{Quellen}

- Alexandre führt sechs zeitgenössische mitteleuropäische Belege auf, worin viermal das Jahr 1180 und zweimal 1179 genannt werden (1990, S. 165). Er wertet aus historischen Gründen das Jahr 1179 als richtig.

- Liège

- Floreffe

- Gand(?)

- Aix

- Brauweiler

- Köln

\section{Hauptquelle}

Annales Brunwilarenses Anno 1152-1179 (MGH SS 16, 1859, S. 728): „1179. Hoc anno terrae motus magnus in kalendis Augusti factus est. Eodem mense eclipsis lune apparuit.“ [1179. In diesem Jahr ereignete sich am 1. August ein grosses Erdbeben. Im selben Monat erschien eine Mondfinsternis.]

\section{Interpretation}

Es gibt keine Schweizer Originalbelege für ein Erdbeben dieses Datums. Die Lokalisierung „Alpnach“ steht in Van Gils \& Leydecker, die Herkunft „Stans“ konnte nicht mehr eruiert werden.

Die zeitgenössischen Quellen stammen vielmehr aus dem nördlichen Mitteleuropa (Brauweiler, Köln, Liège, Floreffe und evt. Gand). Die Angaben zur Mondfinsternis in den Annalen von Brauweiler (MGH SS 16, 1859, S. 728) erlauben gemäss einem Gutachten von Luca Quaglia, Paris, eindeutig den Schluss, dass das Ereignis auf den 1. August 1179 zu datieren ist. Es gibt keine Hinweise auf Schäden in Mitteleuropa. 


\section{Bemerkungen}

1179. Astronomisches Gutachten von Luca Quaglia, Physiker, Paris, für V. Masciadri:

L'éclipse de lune fixe la date au 1/08/1179. Le 19/08/1179 une éclipse totale de lune fût entièrement visible de Cologne $\left(50^{\circ} 56^{\prime} \mathrm{N}, 6^{\circ} 57^{\prime} \mathrm{E}\right)$. Les temps de différents contacts furent:

- début phase partielle $00 \mathrm{~h} 46 \mathrm{~m}$

- début phase totale $01 \mathrm{~h} 50 \mathrm{~m}$

- maximum $02 \mathrm{~h} 43 \mathrm{~m}$

- fin phase totale $03 \mathrm{~h} 35 \mathrm{~m}$

- fin phase partielle $04 \mathrm{~h} 39 \mathrm{~m}$

Ces temps sont exprimés en heure "locale“ de Cologne, c'est-à-dire qu'ils tiennent compte de la différence de temps par rapport au méridien zéro $\left(6^{\circ} 57^{\prime} \mathrm{E}=28 \mathrm{~m}\right)$ et de la différence entre temps terrestre et temps universel (DeltaT $=14 \mathrm{~m}$ ). La lune se leva à $19 \mathrm{~h}$ $21 \mathrm{~m}$ et elle se coucha à $05 \mathrm{~h} 00 \mathrm{~m}$, donc elle était bien visible dans le ciel. Le soleil se leva à $04 \mathrm{~h} 47 \mathrm{~m}$ et l'aurore (au sens „civil“) commença à $04 \mathrm{~h} 06 \mathrm{~m}$. Cette éclipse fût pratiquement centrale (le centre de la lune passa très près du centre de l'ombre terrestre) et la plus longue du siècle. Au milieu de l'éclipse la couleur de la lune était probablement d'un rouge extrêmement foncé.

Tout colle bien avec la description: éclipse qui commence autour de minuit et qui se termine dans les lueurs de l'aube. En 1180 aucun éclipse de lune fût visible de Cologne le mois d'août.

\section{Bemerkung}

Vgl. das Erdbeben vom 1. August $1179 \mathrm{im}$ ECOS-09.

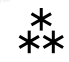

\begin{tabular}{|c|c|c|}
\hline $\begin{array}{l}\text { Datum } \\
\text { 1201, 4. Mai }\end{array}$ & $\begin{array}{l}\text { Zeit } \\
15 \text { Uhr }\end{array}$ & $\begin{array}{l}\text { Ort } \\
\text { - Bayern, D, } \\
\text { - Steiermark, A }\end{array}$ \\
\hline
\end{tabular}

\section{Bewertung des Ereignisses}

Kein Erdbeben: Lokalisierungsfehler.

\section{Korrektur}

4. Mai 1201, 10 Uhr, am Katschberg in Kärnten, A 


\section{Publiziert in:}

- Alexandre (1990)

- $\quad$ Hammerl (1995)

\section{Zusammenfassung}

Beim Erdbeben vom 4. Mai 1201 handelt es sich um ein österreichisches Ereignis, wofür keine schweizerischen Beobachtungen gefunden worden sind. Die Intensität wird auf Grund von Schadenmeldungen von Alexandre sehr hoch eingeschätzt (Alexandre 1990, S. 247: Ix =9-10); Hammerl (1995) hält trotz der 17 zeitgenössischen Quellen die Basis für zu knapp, um eine maximale Intensität zu bestimmen, die Epizentralintensität wird auf 9 geschätzt.

\section{Bemerkungen}

Vgl. das Erdbeben am 4. Mai 1201 am Katschberg in Kärnten, A.

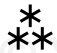

\begin{tabular}{l|l|l} 
Datum & Zeit & Ort \\
1201, 4. Mai & $10 \mathrm{Uhr}$ & Katschberg, Kärnten, A
\end{tabular}

\section{Bewertung des Ereignisses}

Sicheres Ereignis.

\section{Parameter nach ECOS}

Ix: -

\section{Publiziert in:}

- Alexandre (1990)

- Hammerl (1995)

\section{Zusammenfassung}

Beim Erdbeben vom 4. Mai 1201 handelt es sich um ein österreichisches Ereignis, wofür bisher keine schweizerischen Beobachtungen gefunden worden sind. Früher wurde die Intensität auf Grund von Schadenmeldungen sehr hoch eingeschätzt (Alexandre 1990, S. 247: Ix =9-10), heute hält Hammerl (1995) trotz der 17 zeitgenössischen Quellen die Basis für zu knapp, um eine maximale Intensität zu bestimmen, die Epizentralintensität wird auf 9 geschätzt.

$$
\text { ** }
$$




\section{Datum \\ 1215, 21. Mai \\ Zeit (UTC) \\ Ort \\ Nicht lokalisierbar \\ (Nordostschweiz)}

\section{Bewertung des Ereignisses}

Unsicher.

\section{Parameter nach ECOS}

Ix: verspürt

\section{Überlieferung}

Keine; neuer Fund.

\section{Quelle}

- Manuskript: LAAI: Missale der Pfarrkirche Appenzell, o. Sign.

- Druck: Von Euw, A. \& Bischofberger, H., Hsg. (2004): Das Appenzeller Missale:

Text:

Anno dominice incarnationis $\mathrm{m}^{\circ} \cdot \mathrm{cc}^{\circ} \cdot \mathrm{x}^{\circ} \cdot \mathrm{v}^{\circ} \cdot \mathrm{xii}^{\circ}$. kalendas Junii factus est terremotus circa secundum gallicinium.
Im Jahre des Herrn, 1215 am 21. Mai, ereignete sich ein Erdbeben um den zweiten Hahnenschrei.

\section{Interpretation}

Paläographischer Kommentar: Randglosse neben Heiligenliste. Die Randbemerkungen stammen von verschiedenen Händen, teilweise deutlich später als die ursprüngliche Liste. Nach der Anordnung der Texte zu schliessen, muss der Erdbebenvermerk eine der ersten Glossen gewesen sein, er steht auch der Schrift der Heiligenliste noch sehr nahe.

Weitere Auskünfte von Herrn Bischofberger (Appenzellisches Landesarchiv, 2002): Über die Herkunft des Manuskripts sind sich die Bearbeiter noch nicht ganz einig geworden; gesichert ist aber der Raum Chur/St. Gallen/Mainau, allenfalls Schaffhausen. Eine genauere Analyse der Heiligenliste, die viele Besonderheiten enthält und liturgiegeschichtlich von europäischer Bedeutung ist, scheint darüber weitere Aufschlüsse zu versprechen. Der Eintrag über das Erdbeben ist völlig isoliert, das Manuskript enthält keine weiteren chronikalischen Daten, bloss vereinzelte Appenzeller Flur- und Personennamen.

Von Euw hält den Erdbebeneintrag nach Schrift und Inhalt jedenfalls für zeitgenössisch. (2004.) Da keine weiteren Quellen diese Beobachtung bestätigen, wird sie vorläufig mit „unsicher“ und „verspürt“ gewertet. Der zweite Hahnenschrei wird hier mit zwei Uhr morgens übersetzt, also 1 Uhr UTC; vgl. Grotefend, der den Bereich mit „Zeit nach Mitternacht“ festlegt. Dohrn (1996, S. 19) fixiert den Hahnenschrei auf die Zeit vor der Morgendämmerung, vermutlich stützt er sich dabei aber auf eine nicht belegbare Annahme in Niermeyer (1954-76). 
Makroseismische Parameter

\begin{tabular}{|l|l|l|l|l|l|l|}
\hline Ortschaft & Länge & Breite & $I_{\min }$ & $I_{\max }$ & $I_{w}$ & Datenqualität \\
\hline Appenzell (?) & 47.33 & 9.41 & & & verspürt & sehr schwach \\
\hline
\end{tabular}

**

\begin{tabular}{l|l|l} 
Datum & Zeit & Ort \\
1239, September & - & Colmar
\end{tabular}

Bewertung des Ereignisses

Kein Erdbeben: Lokalisierungsfehler.

\section{Korrektur}

18. September 1239, Beobachtungen in Lausanne und Strassburg.

\section{Überlieferung u. a. in:}

- Langenbeck (1892)

- Sieberg (1940)

- Rothé und Schneider (1968)

- Alexandre (1990)

- MECOS-99

- SisFrance (20. Juli 2009)

\section{Quellen}

- Annales Basileenses et Colmarienses (MGH, SS 17, 1861, S. 183-221), darin in den Annales Colmarienses Minores, die sich auf eine Strassburger Quelle stützen (S. 189): „1239. Ecclipsis lunae accidit in mense Maio. Terraemotus factus est in mense Septembri.“ [1239. Eine Mondfinsternis hat sich im Mai ereignet. (Wahrscheinlich ist aber die Mondfinsternis vom 3. Juni gemeint.) Ein Erdbeben ereignete sich im Monat September.]

- Cononis praepositi Lausannenses Notae (MGH SS 24, 1879, S. 781-793): „Eodem anno, quartodecimo kalendas octobris, fuit terremotus." [In demselben Jahr (1239), am 18. September, ereignete sich ein Erdbeben.]

\section{Interpretation}

Der Sitepunkt Colmar ist falsch, denn diese Beobachtung aus einer Colmarer Quelle gehört nach Strassburg. Somit steht eine zeitgenössische Quelle aus Lausanne und eine Abschrift aus Strassburg zur Verfügung, die eine Erdbebenbeobachtung für den September 1239 melden. Von der Lausanner Quelle stammt die Tagesbezeichnung 18. September. Die Jahresdatierung ist zusätzlich durch eine Mondfinsternis gesichert. Hinweise auf 
Schäden gibt es keine. Eine Lokalisierung ist mit dieser Quellenlage nicht machbar. Auch SisFrance (20.7.2009) lokalisierte das Beben mit Fragezeichen, und zwar im Sundgau.

\section{Bemerkungen}

Vgl. im ECOS-09 das Erdbeben vom 18. September 1239 in Lausanne/Strasbourg (nicht lokalisierbar).

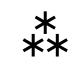

\begin{tabular}{|l|l|l}
\hline Datum & Zeit (UTC) & Ort \\
\hline 1258, 18. Februar & Um 17 Uhr & Nicht lokalisierbar (Chur)
\end{tabular}

\section{Bewertung des Ereignisses}

Unsicher.

\section{Parameter nach ECOS}

Ix: verspürt

\section{Überlieferung}

- $\quad$ Alexandre (1990)

- $\quad$ Guidoboni (2005b)

\section{Quelle}

Nota Curiensis (MGH, Necrologie, 1, 1888, S. 623; und Brunold \& Muraro, 2008): „Hic auditus est terremotus anno 1258, prima indictione [...] in completorium cantabatur in choro Curiensi." [Man hörte hier in der ersten Indiktion ${ }^{13} \mathrm{im}$ Jahr 1258 ein Erdbeben, während im Chor der Kathedrale von Chur die Komplet gesungen wurde.] Die Notiz ist unter „Februarius, 18, XII kal.“ eingeordnet.

\section{Interpretation}

Mit einer einzigen zeitgenössischen Beobachtung aus Chur kann dieses Erdbeben nicht gesichert ausgewertet werden. Ausserdem ist die Tagesdatierung nicht ganz eindeutig abschätzbar. Deshalb ist es auch unklar, ob ein Zusammenhang mit dem neugefundenen Schadenbeben von Barrea in den Abruzzen vom 19. Februar 1258 um 16 Uhr 30 UTC, Intensität 9, besteht (vgl. Guidoboni, 2005b, S. 270-271).

Beim Komplet-Singen handelt es sich um das letzte Gebet im katholischen Tagesablauf, es wurde vor Sonnenuntergang abgehalten, der am 18. Februar um 17 Uhr 51 MEZ, also 16 Uhr 51 UTC, stattfindet; aufgerundet auf 17 Uhr UTC.

\footnotetext{
13 Kalenderzyklus.
} 
Makroseismische Parameter

\begin{tabular}{|l|l|l|l|l|l|l|}
\hline Ortschaft & Länge & Breite & $I_{\min }$ & $I_{\max }$ & $I_{\text {w }}$ & Datenqualität \\
\hline Chur & 46.86 & 9.53 & & & verspürt & sehr schwach \\
\hline
\end{tabular}

Datum

1265, 19. Juni

\section{Zeit (UTC)}

$-$
Ort

St. Blasien, D

\section{Bewertung des Ereignisses}

Kein Erdbeben.

\section{Korrektur}

\section{Überlieferung}

- Giessberger (1922)

- Sieberg (1940)

- Rothé und Schneider (1968)

- MECOS-99

\section{Quellen}

Weder für das erste (19. Juni) noch für das zweite Datum (20. Mai) sind zeitgenössische Quellen nachgewiesen.

\section{Interpretation}

Sieberg (1940) datiert auf den 19. Juni 1265 und stützt sich dabei auf Giessberger (1922). Bei letzterem steht aber: „... item 13. Kal. Junii factus est magnus terrae motus hora matutinali, secutaque est eclipsis solis post meridiem." [Im weiteren geschah ein grosses Erdbeben am 20. Mai, zu morgendlicher Stunde, und es folgte eine Sonnenfinsternis nach dem Mittag.] Dabei stützt er sich auf Steinhofers Chronik, dieser wiederum auf eine alte, unbekannte Chronik von St. Blasien.

Aber auch der 20. Mai ist nicht gesichert. Denn der Sonnenfinsternis-Katalog der NASA (Five Millennium Catalog of Solar Eclipses; http://eclipse.gsfc.nasa.gov/SEcat5/SE1299-1200.html., 24. Aug. 2009) führt erst für den 27. Juni 1265, julianische Datierung, eine Sonnenfinsternis auf. Somit ist die Datierung der Nachricht von Sieberg zumindest vorläufig als Falschmeldung zu werten.

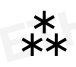




\begin{tabular}{l|l|l} 
Datum & Zeit & Ort \\
\hline 1277, 27. Mai & - & Konstanz
\end{tabular}

\section{Bewertung des Ereignisses}

Kein Erdbeben: Datierungsfehler.

\section{Korrektur}

1277, 9. Juni (-12. Juni)

\section{Überlieferung}

- Rasch (1591)

- Dacher (1470):

- Originalmanuskript in StibSG: Codex 646

- Er stützt sich auf Stetter, Konstanzer Chronist um 1400, der nur in dieser Bearbeitung überliefert ist.

- Dacher ist teilweise ediert in Ruppert (1891), Gesamtedition von Wolff (2008).

\section{Interpretation}

- Rasch-Text (1591): 1277 27. May. 12. gross Erdbiden zu Costnitz am Podensee/in 14. Tagen nacheinander/auch schier durch gantz Teutschlandt.

- Dacher (um 1470 entstanden), nach Wolff (2008): (...) By regierung des bischoffs, als man zalt von der geburt Cristi 1277 jar der V. idus des monats Brachat (9. Juni, Mittwoch), desselben tags kam zwürend (zweimal) erdbidemi, des nachgeenden fritags (11. Juni) kam zu sechsmalen erdbidemi und mornend uff den sambstag (12. Juni) zu nacht ains und also in drü tagen kam ze nünmalen erdbewegung. Das ist vor nie me gehört worden, und darnach uff sambstag nächt (12. Juni oder 19. Juni?) kam sy zu drü malen.

Das Datum 27. Mai aus der Rasch-Kompilation von 1591 ist sonst nirgends zu finden, sodass der 9. Juni bei Dacher von 1470 (der sich auf Stetter, um 1400 stützt) etwas wahrscheinlicher ist, wenn auch immer noch sehr unsicher (vgl. Kommentar zum Ereignis vom 9. Juni 1277). Es lässt sich auch noch spekulieren, ob Rasch oder ein unbekannter Vorgänger die Iden mit den Kalenden verwechselt hat.

\section{Bemerkungen}

Vgl. das Erdbeben vom 9. Juni 1277.

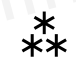




\begin{tabular}{l|l|l}
\hline Datum & Zeit & Ort \\
1277, 9. Juni & - & Nicht lokalisierbar \\
& & (Konstanz)
\end{tabular}

\section{Bewertung des Ereignisses}

Unsicheres Ereignis.

\section{Parameter nach ECOS}

Ix: verspürt

\section{Überlieferung}

- StibSG: Codex 646: Dacher (um 1470), vgl. auch die Editionen von Ruppert (1891) und Wolff (2008)

- Stumpf (1548)

- Rasch (1591)

- Volger (1857)

- Ruppert (1891), stützt sich u.a. auf Dacher

- Giessberger (1922)

- Sieberg (1940)

\section{Quellen}

Bisher sind keine zeitgenössischen Beobachtungen nachweisbar, auch nicht in den Annales Basileenses et Colmarienses (MGH, SS, 17, 1861), deren Verfasser für Naturphänomene sehr sensibilisiert ist.

\section{Interpretation}

Zwei wichtige Kompilatoren beschreiben eine Erdbebenserie im Juni 1277. Ruppert (1891), der sich unter anderem auf die Konstanzer Gebhard Dacher (um 1400-1450) und Johannes Stetter (bis 1399) stützt, schreibt von neun Erdbeben in der Zeit vom 9. bis 12. Juni 1277. Stumpf (1548, fol. 58) datiert zwölf Erdbeben auf die Tage zwischen dem 6 . und 20. Juni, die Formulierungen scheinen unabhängig zu sein. Schäden werden keine erwähnt.

Ohne zeitgenössische Belege müssen diese Aussagen als „unsicher“ gewertet werden (ebenso die nur bei Ruppert, aufgrund von Stetter, 14. Jh., aufgeführte Erdbebenserie vom 10. Februar bis 28. März 1277). Über einen allfälligen Zusammenhang mit dem angeblichen Beben von 1279 kann nur spekuliert werden (vgl. unten).

\section{Makroseismische Parameter}

\begin{tabular}{|l|l|l|l|l|l|l|}
\hline Ortschaft & Länge & Breite & $I_{\min }$ & $I_{\max }$ & $I_{\text {w }}$ & Datenqualität \\
\hline Konstanz & 47.67 & 9.18 & & & verspürt & sehr schwach \\
\hline
\end{tabular}




\section{Bemerkungen}

Vgl. auch die Meldung über ein angebliches Erdbeben am 2. September 1279.

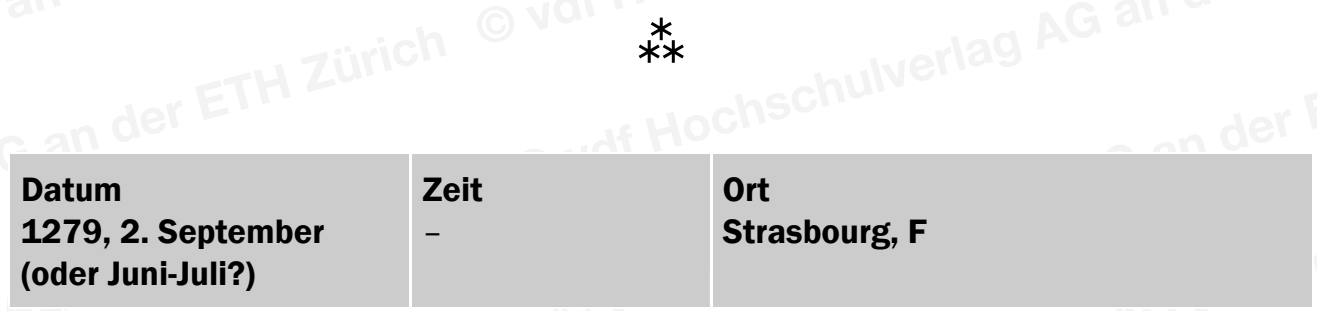

\section{Bewertung des Ereignisses}

Kein Erdbeben.

\section{Korrektur}

Kein Korrekturvorschlag.

\section{Überlieferung u. a. in:}

- Langenbeck (1892)

- Giessberger (1922)

- Sieberg (1940)

- Rothé und Schneider (1968)

- Leydecker (1986)

- SisFrance

\section{Quellen}

Zwei Quellenhinweise:

- In den Annales Colmarienses maiores (MGH SS 17, 1861, S. 205) steht zwischen den Datierungen 12. Juni und 14. Juli 1279: „Et terremotus ecclesias et castra multa subvertit." [Und ein Erdbeben hat Kirchen und viele Burgen umgestürzt.] Diese Annalen sind jedoch nur in Kopien aus dem 15. oder 16. Jahrhundert überliefert.

- Fritsche (Friedrich) Closener's Chronik (1870, S. 136): „September 2. Do man zalt 1279 jor, an dem nehesten dag noch sant Gilgen dag, do kam ein ertbideme.“ Ein Quellenverweis fehlt.

Kein weiterer Hinweis in den Elsässer Quellen der MGH SS 17 (1861):

- Ellenhardi Argentinensis Annales 1261-1294

- Annales Colmarienses et Basileenses

- Annales Argentinenses

Hinweise für 1289:

- Ellenhardi Chronicon (MGH SS 17, S. 132): „1289, Sept. 24: anno domini ut supra sabbatho post Mathei apostoli (...) terre motus factus est magnus in civitate Argen- 
tinensi, ita quod columpne templi pre nimio impeto terre viderunter moveri ... ." [Im Jahr des Herrn, das schon oben genannt wurde, am Samstag nach dem Tag des Apostels Matthäus ... ereignete sich ein grosses Erdbeben in der Stadt Strassburg, derart, dass die Säulen des Münsters sich durch eine grosse Kraft zu bewegen schienen.] Diese Chronik enthält aber auffallenderweise keinen Hinweis auf 1279.

- Fritsche Closener: S. 53: „1289. Ein ertbidem. Donoch in demselben jor an dem samsdag noch sant Matheus dag (24. September), do kam zuo Strosseburg ein also grosser ertbideme daz die sullen in dem munster wagetent alse sere, daz man vorhte, daz munster wolte vallen.“

\section{Interpretation}

Leydecker (1986, S. 23) bewertet die Hinweise auf den 2. September 1279 mit einer Intensität 8. Die vorhandenen Dokumente lassen jedoch keine sicheren Schlüsse auf ein Schadenbeben zu. Denn die ursprünglich zeitgenössischen Annales Colmarienses maiores berichten zwar von einem grossen Schadenbeben zwischen dem 12. Juni und dem 14. Juli 1279, doch sind sie nur in Kopien des 15. und 16. Jahrhunderts überliefert. Die Kompilation von Closener aus dem Jahr 1362 nennt den 2. September 1279 als Datum für ein Beben ohne Schäden. Hinweise aus zeitgenössischen Quellen, z. B. in den elsässischen Quellen der MGH SS 17, sind aber nicht zu finden, ausser für ein Beben ohne Schäden im Jahr 1289.

Da die Colmarer Annalen grosse Schäden nennen, müssten sich eigentlich in diesem Jahrhundert bereits mehrere Quellen dazu finden lassen, ähnlich dem Churwaldner Beben von 1295. Deshalb könnte es sich bei der entsprechenden Manuskriptstelle auch um eine Verwechslung der Kopisten im 15. und 16. Jahrhundert mit dem Basler Beben von 1356, wo nachweislich Kirchen und Burgen beschädigt wurden, oder mit 1289 handeln. Ob ein Zusammenhang mit dem Erdbeben vom 9. Juni 1277 besteht, ist unklar. Darum ist eher auf eine Falschmeldung zu schliessen. Trotzdem sollten all diese Daten bei weiteren Quellensuchen im Auge behalten werden.

\section{Bemerkungen}

Vgl. auch die Meldungen über unsichere Erdbeben am 9. Juni 1277 und 24. September 1289.

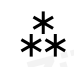

\begin{tabular}{l|l|l} 
Datum & Zeit & Ort \\
1 1280, 26. Oktober & - & $\begin{array}{l}\text { Nicht lokalisierbar } \\
\text { (Colmar, F) }\end{array}$
\end{tabular}

\section{Bewertung des Ereignisses}

Sehr unsicheres Ereignis. 


\section{Parameter nach ECOS}

Ix: verspürt

\section{Überlieferung u. a. in:}

- Langenbeck (1892)

- Rothé und Schneider (1968): mit dem Datum 25. Oktober

- SisFrance

Nicht vorhanden in:

- Bertrand (1756)

- Volger (1857)

- MECOS-99

\section{Quelle}

Annales Colmarienses Maiores (MGH SS 17, 1861, S. 207): „Septimo kalendas Novembris (26. Oktober) fuit terremotus." [Am 26. Oktober 1280 ereignete sich ein Erdbeben.]

\section{Interpretation}

Die Colmarer Annalen sind im allgemeinen als zuverlässige Quelle zu bezeichnen, doch ohne zusätzliche Belege kann dieses Erdbeben noch nicht definitiv ausgewertet werden. Da keine Schäden erwähnt werden, wurde die Intensität mit „verspürt“ gewertet.

\section{Makroseismische Parameter}

\begin{tabular}{|l|l|l|l|l|l|l|}
\hline Ortschaft & Länge & Breite & $I_{\min }$ & $I_{\max }$ & $I_{\text {w }}$ & Datenqualität \\
\hline Colmar & 48.09 & 7.37 & & & verspürt & sehr schwach \\
\hline
\end{tabular}

\section{***}

\begin{tabular}{l|l|l} 
Datum & Zeit & Ort \\
1290 & - & Schweiz/Innertkirchen, BE
\end{tabular}

\section{Bewertung des Ereignisses}

Kein Erdbeben: Lokalisierungsfehler.

\section{Korrektur}

Möglicherweise handelt es sich um eine Verwechslung mit Lissabon, P. 


\section{Überlieferung u. a. in:}

- Bertrand (1756)

- Guéneau de Montbelliard (1761)

- Volger (1857)

- Giessberger (1922)

- $\quad$ Gils, Van \& Leydecker (1991)

- MECOS-99

Nicht vorhanden in:

- Rasch (1591)

- SisFrance (24. September 2009)

\section{Interpretation}

Bertrand (1756), S. 242: „1290 ..., \& en mille deux-cent quatre-vingt-dix, on essuya des Tremblemens de terre, qui furent à-peu-près universels. La Suisse n'en fut point exempte.“

Es sind jedoch keine Schweizer Originalquellen für das Jahr 1290 bekannt. Bertrand ist der erste, der auf ein Erdbeben in der Schweiz im Jahre 1290 aufgrund einer unbekannten Quelle, seismologisch nicht nachvollziehbar, schliesst. Innertkirchen wird erst seit Van Gils \& Leydecker als Epizentrum genannt.

Auch in den zeitgenössischen Annales Colmarienses Maiores ist dieses Beben nicht verzeichnet. Vielmehr deutet die Formulierung Bertrands auch eine Verwechslung mit dem 1290-Erdbeben von Lissabon [(Volger (1857), Martins \& Mendes (2001)] an.

\section{**}

\begin{tabular}{l|l|l} 
Datum & Zeit & Ort \\
\hline 1295, 3. April & - & Nicht lokalisierbar (Colmar, F)
\end{tabular}

\section{Bewertung des Ereignisses}

Unsicher.

\section{Parameter nach ECOS}

Ix: 5

\section{Überlieferung}

- $\quad$ Volger (1857)

- Amberg (1892) 


\section{Quellen}

- Annales Colmarienses Maiores (MGH SS 17, 1881, S. 221): „Tertio Nonas Aprilis fuit in Briscaugia et in Alsacia vehemens terrae motus." [Am 3. April 1295 ereignete sich im Breisgau und im Elsass ein starkes Erdbeben.]

- Gesta episcoporum Mettensium 1295-97 (MGH, SS, 10, 1852, S. 551)

\section{Interpretation}

Die einzige zeitgenössische Quelle, die Colmarer Annalen, ist für Naturphänomene sehr aufmerksam. Doch ist eine Vermischung mit den Überschwemmungen, die die Kantone Basel in diesem Jahr betroffen haben (vgl. auch die Gesta episcoporum Mettensium, 1852), oder mit dem Beben vom 3. September dennoch möglich. Ohne zusätzliche zeitgenössische Beobachtungen muss deshalb dieses Erdbeben mit „unsicher“ gewertet werden.

\section{Makroseismische Parameter}

\begin{tabular}{|l|l|l|l|l|l|l|}
\hline Ortschaft & Länge & Breite & $I_{\min }$ & $I_{\max }$ & $I_{\text {w }}$ & Datenqualität \\
\hline Elsass & 48.21 & 7.44 & 5 & & & sehr schwach \\
\hline Breisgau & 47.93 & 7.97 & 5 & & & sehr schwach \\
\hline Colmar & 48.09 & 7.37 & 5 & & 5 & sehr schwach \\
\hline
\end{tabular}

\begin{tabular}{l|l|l} 
Datum & Zeit & Ort \\
\hline 1295, 5. April & - & Chur und Reichenau, GR
\end{tabular}

\section{Bewertung des Ereignisses}

Keine Erdbeben: Datierungsfehler (und Lokalisierungsfehler).

\section{Korrektur}

- 1295, 3. April (Colmar, F)

- $\quad 1295,3$. September, in Churwalden, GR

\section{Überlieferung u. a. in:}

- Volger (1857)

- Candreia (1905)

- Sieberg (1940)

- Gils, Van \& Leydecker (1991)

- MECOS-99

- SisFrance (10. Sep. 2009) 
Wahrscheinlich Vermischung der Hinweise aus:

- Annales Colmarienses maiores (MGH SS 17, 1861, S. 202ff.)

- Annales Osterhovenses (MGH SS 17, 1861, S. 538-58)

\section{Interpretation}

Wahrscheinlich wurde die Datierung in den Annales Colmarienses maiores falsch interpretiert. Die Lokalisierung „Reichenau, GR“ stammt aus Van Gils \& Leydecker (1991) und geht vermutlich auf eine Verwechslung und Vermischung mit dem April-Beben in Colmar und dem September-Beben in Churwalden zurück.

\section{Bemerkungen}

Vgl. den Eintrag von SisFrance zum 3. April 1295 und im ECOS-09 das Erdbeben vom 3. September 1295, Churwalden.

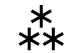

\begin{tabular}{|l|l|l}
\hline Datum & Zeit & Ort \\
1295, 3. September & - & Churwalden, GR
\end{tabular}

\section{Bewertung des Ereignisses}

Sicheres Ereignis.

\section{Parameter nach ECOS}

Ix: 8

\section{Publiziert in:}

- Albini (1994)

- Gollnick (2001 und 2002)

- Schwarz-Zanetti et al. (2004)

- Gisler et al. (2005)

- Schwarz-Zanetti (2008b)

\section{Zusammenfassung}

Mehrere zeitgenössische Quellen belegen, dass am Nachmittag des 3. September 1295 ein starkes Erdbeben die Ostschweiz, Süddeutschland, Österreich und Italien erschütterte. Das Epizentrum lag in der Gegend von Churwalden im Kanton Graubünden. Dabei wurden das dortige Kloster und Burgen der weiteren Umgebung stark beschädigt, was auf eine maximale Intensität von 8 schliessen lässt. Es handelt sich hier um das älteste Schweizer Schadenbeben, das sich aufgrund mehrerer Schriftquellen zuverlässig beschreiben lässt. 
Makroseismische Parameter

\begin{tabular}{|l|l|l|l|l|l|l|}
\hline Ortschaft & Länge & Breite & $I_{\min }$ & $I_{\max }$ & $I_{\text {w }}$ & Datenqualität \\
\hline Tumegl/Tomils & 46.76 & 9.44 & 6 & & & sehr schwach \\
\hline Landshut & 48.54 & 12.15 & & verspürt & sehr schwach \\
\hline Monza & 45.58 & 9.27 & & verspürt & sehr schwach \\
\hline Domleschg & 46.76 & 9.44 & & verspürt & sehr schwach \\
\hline Wien & 48.22 & 16.38 & & verspürt & sehr schwach \\
\hline Heilsbronn & 49.34 & 10.79 & & verspürt & sehr schwach \\
\hline Chur & 46.86 & 9.53 & 7 & 8 & 8 & sehr schwach \\
\hline Churwalden & 46.78 & 9.54 & 7 & & 8 & sehr schwach \\
\hline Kanton Graubünden & 46.61 & 9.57 & 7 & 9 & 8 & schwach \\
\hline Gams & 47.2 & 9.44 & 6 & 8 & 7 & sehr schwach \\
\hline Bergamo & 45.7 & 9.68 & 6 & 8 & 6 & sehr schwach \\
\hline Konstanz & 47.67 & 9.18 & 5 & 6 & 6 & mässig \\
\hline Diessen am Ammersee & 47.94 & 11.1 & 4 & & 5 & sehr schwach \\
\hline St. Florian & 48.14 & 14.37 & 4 & & 5 & sehr schwach \\
\hline
\end{tabular}

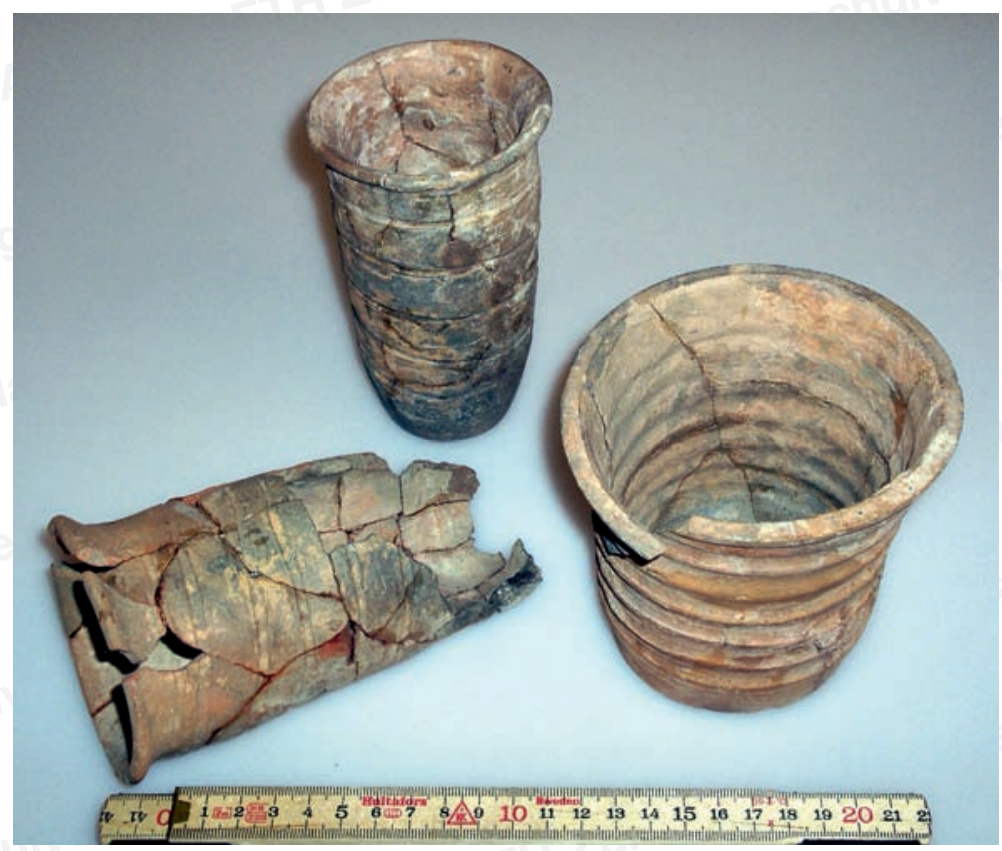

Abbildung 1: Becherkacheln aus den Konventbauten von Churwalden, 12.-13. Jahrhundert (Archäologischer Dienst Graubünden) 
Diese Becherkacheln eines Ofens aus dem 12.-13. Jahrhundert aus den Churwaldner Konventbauten wurden bei Ausgrabungen in den Jahren 1962-64 gefunden. Über die Ursache der Beschädigungen kann nur spekuliert werden, ein Zusammenhang mit dem Erdbeben von 1295 ist jedoch nicht ausgeschlossen. (Vgl. auch Bergmann, 1997; Tauber 1980; Foto: M. Schwarz, Wetzikon, 2006)

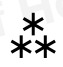

\begin{tabular}{|l|l|l}
\hline Datum & Zeit & Ort \\
1295, 4. September & - & Chur
\end{tabular}

\section{Bewertung des Ereignisses}

Kein Erdbeben: Datierungsfehler (und Lokalisierungsfehler).

\section{Korrektur}

3. September 1295 in Churwalden

\section{Überlieferung u. a. in:}

- Volger (1857)

- Candreia (1905)

- MECOS-99

\section{Quellen}

4. September

- Annales Osterhovenses, MGH, SS, 17 (1861), S. 538-58

- $\quad$ Continuatio Vindobonensis, MGH, SS, 9 (1851), S. 699-722

\section{September}

- Stiftsbibliothek St. Gallen, Codex 646: Dacher, Gebhard (1470), Wolff (2008)

- Notae Diessenses, MGH SS 17 (1861), S. 324-327

- Camassi (o. J.): Zitiert einen Augenzeugenbericht aus Monza.

\section{Interpretation}

Annales Osterhovenses:

„1295. in die pasce quod tunc evenit in vigilia Ambrosii, cecidit nix hora matutina ad spissitudinem erecte manus, et permansit usque ad horam diei nonam. Eodem anno (1295) in octava sancti Augustini (28. Aug. - also am 4. Sept.) per terre motum claustrum ordinis Premonstratensis Churbaldia et quindecim castra in eodem confinio funditus corruerunt.“ [1295 Am Tag von Ostern, das damals auf den Tag vor S. Ambrosius fiel, fiel am Morgen Schnee so hoch wie eine ausgestreckte Hand und blieb bis zur neunten Stunde des Tages liegen. Im selben Jahr, am 8. Tag nach S. Augustinus, wurden durch 
ein Erdbeben das Kloster der Prämonstratenser in Churwalden und fünfzehn Burgen in derselben Gegend bis auf den Grund zerstört.]

Die Annales Osterhovenses und die Continuatio Vindobonensis sind die einzigen, die auf den 4. September datieren, auf die Osterhofener Annalen stützen sich auch die aufgeführten Kompilationen. Die drei anderen Quellen, die ein Tagesdatum angeben, nennen den 3. September, sodass eher auf dieses Datum zu schliessen ist (vgl. oben, 3. September 1295).

\section{Bemerkungen}

Vgl. das Erdbeben vom 3. September 1295.

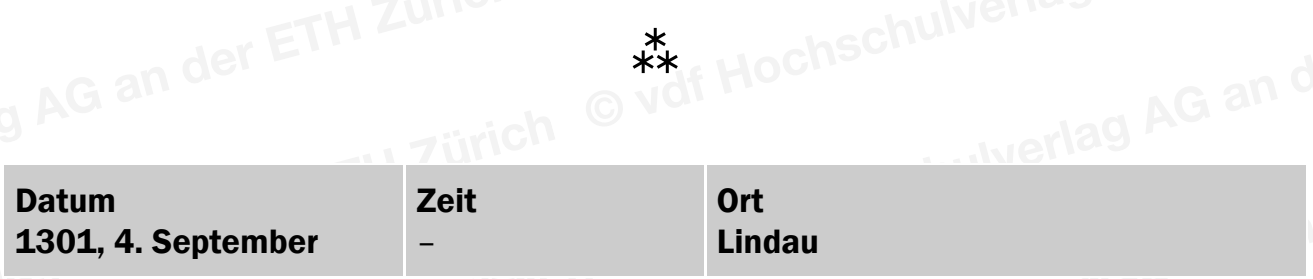

\section{Bewertung des Ereignisses}

Kein Erdbeben: Datierungsfehler.

\section{Korrektur}

Eventuell 3. September 1295, Churwalden

\section{Überlieferung u. a. in:}

- Wolfart (1909/1979): S. 278

- Wolfart (1912): S. 29f

- Giessberger(1922): S. 32

- MECOS-99

\section{Interpretation}

Der Hinweis stammt aus Wolfart (1909 und 1912) und wird später auch von Giessberger aufgenommen. Es lassen sich aber keine Augenzeugenberichte nachweisen. Hingegen lässt die Tagesdatierung mit einer gewissen Wahrscheinlichkeit auf eine Verwechslung mit dem Beben vom 3. September 1295 schliessen: „1301. am 4. Septembris war ein Erdbidem, der tat hin und wider an den Gebäuen grossen Schaden." Denn dieses Beben wird in einigen Originalquellen auch auf den 4. September datiert.

\section{Bemerkungen}

Vgl. das Erdbeben vom 3. September 1295 in Churwalden, GR.

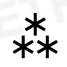




\section{Datum Zeit Ort \\ \begin{tabular}{|l|l|l} 
1305, 21. Oktober & - & Como, I
\end{tabular}}

\section{Bewertung des Ereignisses}

Sehr unsicheres Ereignis.

\section{Parameter nach ECOS}

Ix: -

\section{Überlieferung u. a. in:}

- Baratta (1901), zitiert Tatti, P.L. (1683)

- Carozzo: General catalogue of Italian earthquakes (1973)

- MECOS-99

Nicht vorhanden in:

- Candreia (1905)

- DOM4.1 (21. Juli 2009)

- Guidoboni (2005b)

\section{Quellen}

Es sind keine Schweizer Quellen nachweisbar. Baratta (1901) stützt sich auf L. Tatti (1683): Degli annali Sacri della città di Como racolti e descritti dal P. D. Primo Luigi Tatti can. Regolare della congregazione di Somasca, libro I, pag. 13: „A Como e suo territorio fu sentita una scossa che recò non poco spavento, ma non produsse alcuna rovina negli edifici." [In Como und dessen Umgebung wurde ein Erdstoss verspürte, der nicht wenig Angst verursachte, aber er führte zu keinerlei Schäden an den Gebäuden.]

\section{Interpretation}

Da nur das Quellenzitat von Tatti für Como vorhanden ist, das im Rahmen des vorliegenden Projektes nicht überprüft werden konnte, muss das Ereignis vorläufig als sehr unsicher gewertet werden, obwohl Tatti sich möglicherweise auf eine zeitgenössische Quelle gestützt hatte. Nach dem Wortlaut der Quelle handelte es sich um ein schwaches Beben.

Entscheidend für den ECOS-09 ist aber, dass dieses Beben bisher nicht in Schweizer Quellen und Kompilationen, z. B. Candreia (1905), nachgewiesen werden konnte.

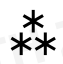




\begin{tabular}{|c|c|c|}
\hline $\begin{array}{l}\text { Datum } \\
1322,16 . \text { Oktober }\end{array}$ & $\begin{array}{l}\text { Zeit } \\
\text { (Morgens) }\end{array}$ & $\begin{array}{l}\text { Ort } \\
\text { Nicht lokalisierbar (Lausanne und } \\
\text { Genève) }\end{array}$ \\
\hline
\end{tabular}

\section{Bewertung des Ereignisses}

Unsicheres Ereignis.

\section{Parameter nach ECOS}

Ix: verspürt

\section{Überlieferung u. a. in:}

- Bertrand (1756)

- Guéneau de Montbelliard (1761)

- Volger (1857)

- MECOS-99

- SisFrance

\section{Quelle}

Fasciculus temporis. Chronica Monasterii S. Victoris Gebennensis? Mallet (1845, S. 305-306):

A. D. mcccxxij. (...) Item eodem anno, die sabbati ante festum B. Luce evangeliste id est xvij. kal. novembris, hora prima, tremuit terra Gebenn(ensis) (Genf) et in locis circumvicinis, et apud Lausannam. [Im Jahre 1322 ... (...) Im selben Jahr, am Samstag vor St. Lukas Evangelist, d. h. am 16. Oktober, in der ersten Stunde (um Mitternacht oder bei Tagesanbruch), bebte die Erde in Genf und Umgebung und bei Lausanne.]

\section{Interpretation}

Für die seit Bertrand (1756) genannte Datierung „Ende November 1322“ eines Genfer Bebens konnte kein zeitgenössischer Beleg gefunden werden; er stützt sich dabei angeblich auf einen Auszug aus einer nicht genannten handschriftlichen Chronik durch Prof. Jalabert von Genf. Letzterer bezieht sich aber möglicherweise auf ein Ereignis im Oktober, das in der zeitgenössischen Chronica Monasterii S. Victoris Gebennensis zu finden ist.

Diese von Mallet edierte Kloster-Chronik aus Genf bezeichnet das Datum gleich zweifach. Der 18.10.1322 war ein Montag, also war der Samstag davor der 16.10., was sich mit der Datierung nach den Kalenden bestätigt. Zusätzlich sind auch die Stunde und ein ungefährer Schütterbereich angegeben. Keine Angabe von Schäden. Für einen Einzelhinweis kann somit nur die Wertung „unsicheres Ereignis“ gegeben werden. 
Makroseismische Parameter

\begin{tabular}{|l|l|l|l|l|l|l|}
\hline Ortschaft & Länge & Breite & $I_{\min }$ & $I_{\max }$ & $I_{w}$ & Datenqualität \\
\hline Lausanne & 46.52 & 6.63 & & & verspürt & sehr schwach \\
\hline Genève & 46.21 & 6.14 & & & verspürt & sehr schwach \\
\hline
\end{tabular}

**

Datum

1322, 31. Oktober

1322, November
Zeit

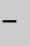

Ort

- Genève

- Genève

\section{Bewertung des Ereignisses}

Kein Erdbeben: Datierungsfehler.

\section{Korrektur}

1322, 16. Oktober

\section{Überlieferung u. a. in:}

- Bertrand (1756): „Sur la fin du Mois de Novembre, mille trois-cent vingt-deux, Genêve essuya un tremblement assez sensible, mais sans causer de dommages."

- Volger (1857)

- Gils, Van \& Leydecker (1991)

- MECOS-99

\section{Interpretation}

Mit grosser Wahrscheinlichkeit handelt es sich in beiden Fällen um einen Datierungsfehler für das Ereignis vom 16. Oktober 1322, das in der von Mallet (1845) publizierten Genfer-Klosterchronik genannt wird. Das Datum „31. Oktober“ stammt aus der Kompilation Van Gils \& Leydecker, die keine Quellenverweise aufführt.

\section{Bemerkungen}

Vgl. im ECOS-09 das Beben vom 16. Oktober 1322 in Genève.

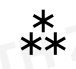




\begin{tabular}{|l|l|l|} 
Datum & Zeit & Ort \\
1334, 4. Dezember & 23 Uhr & $\begin{array}{l}\text { Monte Baldo - } \\
\text { Lago di Garda, I }\end{array}$
\end{tabular}

\section{Bewertung des Ereignisses}

Kein Erdbeben: evtl. Lokalisierungsfehler.

\section{Korrektur}

Eventuell Verona.

\section{Überlieferung}

- Baratta (1901)

- Gils, Van \& Leydecker (1991)

- Rüttener (1995)

- MECOS-99

- Guidoboni (2005b)

- DOM4.1

Nicht vorhanden in mitteleuropäischen Kompilationen wie:

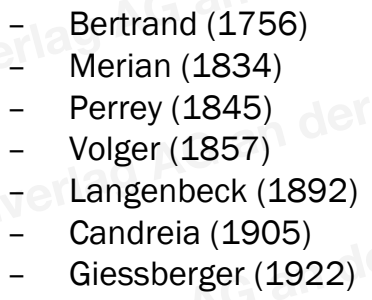

Der zeitgenössische Chronikschreiber Johannes von Winterthur, auch Vitoduranus genannt (ca. 1300-1349), in Lindau am Bodensee hält dieses Verona-Beben von 1334 in seiner Chronik (MGH SRG, Nova Series, Bd. III 1924) nicht fest, nur das früher Villach-, heute Friaul-Beben genannte Erdbeben von 1348 wird bei inm erwähnt.

\section{Interpretation}

In zeitgenössischen Schweizer Quellen konnte das Beben von Verona vom 4. Dezember 1334 bisher nicht nachgewiesen werden. Es wird von Guidoboni (2005b, S. 385-386) aufgrund einer einzigen zeitgenössischen Quelle auf eine Intensität von „6-7?“ geschätzt, obwohl diese keine Schäden erwähnt und die Intensität nur mit "maximus“ umschreibt. Erst in späteren Abschriften (vgl. Guidoboni 2005b) werden Schäden aufgeführt. Der Tag des Erdbebens ist auffallenderweise mit dem Todestag des Papstes Johannes XXII., der in Avignon lebte, verknüpft, sodass zu fragen ist, ob der Chronist das religionspolitische Ereignis überhöhen wollte.

Die Lokalisierung "Monte-Baldo - Lago di Garda“ im MECOS-99 stammt von Van Gils \& Leydecker (1991). Der Eintrag im MECOS-99 erfolgte nur aufgrund von Rüttener (1995). 


\section{Bemerkungen}

Vgl. den italienischen Katalog DOM4.1.

$$
\text { ** }
$$

\begin{tabular}{|l|l|l} 
Datum & Zeit & Ort \\
$1336,8$. Oktober & - & Basel
\end{tabular}

\section{Bewertung des Ereignisses}

Kein Erdbeben.

\section{Korrektur}

\section{Überlieferung u. a. in:}

- Sieberg (1940): enthält den ältesten Hinweis, er gibt aber keine direkte Quelle an.

- MECOS-99, stützt sich auf Sieberg.

\section{Quellen}

Bisher konnte keine zeitgenössische Bestätigung für diese kompilatorische Aussage gefunden werden.

\section{Interpretation}

Da eine zeitgenössische Quelle fehlt, kann in zwei Richtungen spekuliert werden. Es kann sich um eine Verwechslung mit dem Datum des berühmten Basler Bebens von 1356 (8. Oktober 1336 Basel statt 18. Oktober 1356) in Siebergs unbekannter Quelle handeln. Oder das berühmte Datum verdeckt auf unbekannte Weise ein unbekanntes Erdbeben. Somit ist dieses Ereignis vorläufig als Falschmeldung zu werten.

\section{Bemerkungen}

Vgl. das Erdbeben vom 18. Oktober 1356 in Basel.

$$
\text { ** }
$$

\section{Datum}

1346, 24. November

Zeit

\section{Bewertung des Ereignisses}

Kein Erdbeben. 


\section{Korrektur}

Erosionsschaden.

\section{Überlieferung}

Einfall der Pfalz, ohne Begründung der Ursachen:

- Basler Annalen, Kleinere (Bernoulli, 1895), ca. 1370 entstanden, Handschrift wohl aus dem 1. Viertel des 15. Jh.: „Anno domini 1346 an sant Katherinen abend do viel die Phallenz ze Basel hinder unser frouwen munster.“

- Codex Beinheim, Univ. Bibl. Basel, fol. 32: „Anno MCCC und im sechsundvierzigsten an sant Catharinen Tag [25. Nov.] viel die Pfalz hinder dem Münster in Ryn und geschach grosser Schaden“ (zitiert nach E. Wechsler (1987)).

- Sebastian Münster (1544), Verfall der Pfalz ohne Erdbebenhinweis oder andere Begründung.

Einfall der Pfalz, angeblich durch Erdbeben, u. a. in:

- Rasch (1591) enthält die älteste bekannte Behauptung, dass der Zusammensturz der Pfalz durch ein Erdbeben verursacht worden sei und stützt sich dabei angeblich auf Sebastian Münster, der aber, wie bereits oben festgehalten, kein Erdbeben erwähnt.

- Beuther (1601)

- Klauber (1617)

- Gross (1624)

- Bertrand (1756)

- Giessberger (1922)

Neuere Untersuchungen:

- Wechsler (1987), S. 60: „Die Überlieferung machte daraus ein Erdbeben, obwohl es sich auch nur um die Folge einer allmählichen Erdverschiebung gehandelt haben könnte.“

- Reicke: „Beobachtungen zur Baugeschichte der Basler Pfalz“ anlässlich der Sanierung 1994/95. Artikel aus dem Internet, Output vom 20. November 2002, www.archaeobasel.ch/Bucherstand/Jahresbericht/JB94/94Kapitel10.pdf S. 113f. Die Dokumentation liegt bei der Basler Denkmalpflege. Für jüngere Schäden schlägt er Unterspülung und Frostsprengung vor.

Zeitgenössische Quelle:

- Die zeitgenössische Chronik von Johannes von Winterthur (Vitoduranus, 1924) enthält keinen Hinweis auf ein Erdbeben an diesem Datum.

\section{Interpretation}

Die Nachricht vom Zusammensturz der Pfalz von Basel, einer künstlich aufgeschütteten Terrasse zwischen dem Münster und dem nahen Rhein, wurde von Rasch (1591) fälschlicherweise aufgrund von Sebastian Münster als das Resultat eines Erdbebens interpre- 
tiert. Die Ähnlichkeit des Datums 1346 mit dem grossen Basler Beben von 1356 mag auch eine Rolle gespielt haben. Eine Rutschung im Bereich der Pfalz ist aber aufgrund der Basler Annalen wahrscheinlicher. Ein ähnliches Ereignis wird auch für 1502 überliefert. Der Erosionsschaden von 1346 kann auch durch die Jahrtausend-Überschwemmung vom Juli und August 1342 und die starken Niederschläge im Frühling und Sommer 1346 gefördert worden sein (Schwarz-Zanetti, 1992).

\begin{tabular}{l|l} 
& \multicolumn{2}{c}{$\begin{array}{c}* * * \\
\text { Datum } \\
\text { 1348, 25. Januar }\end{array}$} & $\begin{array}{l}\text { Zeit } \\
\text { (Nachmittag) }\end{array}$ & $\begin{array}{l}\text { Ort } \\
\text { Friaul, I, } \\
\text { (früher Villach-Beben, A) }\end{array}$
\end{tabular}

\section{Bewertung des Ereignisses}

Sicheres Ereignis.

\section{Parameter nach ECOS}

Ix: 9.5

\section{Publiziert u. a. in:}

- Borst (1981)

- Hammerl (1992)

- Guidoboni et al. (2005b), S. 403-434: 1348 January 25, Carinthia (southern Austria) and north-eastern Italy. Io = IX-X (S. 433)

Zwei zusätzliche zeitgenössische Quellen:

1. Stiftsarchiv Engelberg, OW, Nekrologium: Cod. 26 fol. 53v: „Me retriplica semel (minus 1 geminata) dat terrae motus Pauli conversio notum; vespera dum canitur tunc agitur, singula terrentur turres castraque moventur." [Übersetzung von G. Heer, 1975, S. 102, Anm. 135: Tausend und dreimal hundert und fünfzig weniger zwei (1348) beschert uns Pauli Bekehrung (25. Jan.) die bekannte Erderschütterung. Während der gesungenen Vesper tritt das Beben auf, Türme und Häuser geraten in Bewegung.]

2. Goswin (1880, S. 135): Chronik des Stiftes Marienberg (Vintschgau, $A^{14}$ ): „Postea in anno domini MCCCXLIIII. tanti fuerunt terremotus et tam validi, quod castra, civitates subverterunt sicut in Karinthia hodierna die cernitur. Istum terremotum secuta fuit pestilencia valida nimis in anno domini MCCCXLVIII, ... . " [Danach, im Jahre 1344 (sic; Druckfehler für 1348), ereigneten sich viele und sehr starke Erdbeben, so dass Burgen und Städte zerfielen, in der gleichen Art, wie man es in Kärnten (A) am heutigen Tag sieht. Diesem Erdbeben ist eine überaus starke Pest gefolgt im Jahre 1348, ... .]

\footnotetext{
14 Heute Vinschgau, Südtirol, I.
} 
Die Erdbebenbeschreibung aus dem Kloster Engelberg zeigt zum grössten Teil die Form eines Hexameters des 14. Jahrhunderts und soll das Ereignis im Gedächtnis fixieren, vergleichbar den gängigen Merkversen zum Erdbeben von 1356.15 Dies ist allerdings auch ein Indiz dafür, dass der Vers als Fremdinformation den Weg nach Engelberg gefunden haben könnte. Doch andererseits kann sich der Schütterradius bei einer Intensität von 9-10 durchaus bis in die Zentralschweiz erstreckt haben, ${ }^{16}$ worauf auch die in Guidoboni (2005b, v. a. S. 432-434) aufgeführte Quelle aus Strassburg bereits hinweist. Die zeitgenössische Quelle aus dem Vinschgau ist trotz der Fehldatierung vertrauenswürdig, sie liegt auch näher am Epizentrum.

Zeitgenössische Erdbebentheorie:

Konrad von Megenberg verfasste in den Jahren 1348-1350 sein deutschsprachiges „Buch der Natur“, das auch ein Kapitel über Erdbeben mit einem Hinweis auf das Villach/Friaul-Beben von 1348 enthält (Luff \& Steer, 2003), er stützt sich dabei zum grössten Teil auf den „Liber de natura rerum“ von Thomas von Cantimpré (1241), einem Schüler von Albertus Magnus. Letzterer griff auch auf griechische, arabische und jüdische Kommentare zurück.

\section{Makroseismische Parameter (aus der Schweiz)}

\begin{tabular}{|l|l|l|l|l|l|l|}
\hline Ortschaft & Länge & Breite & $I_{\min }$ & $I_{\max }$ & $I_{\text {w }}$ & Datenqualität \\
\hline Engelberg & 46.82 & 8.4 & 3 & 5 & 4 & sehr schwach \\
\hline
\end{tabular}

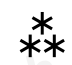

\begin{tabular}{l|l|l} 
Datum & Zeit & Ort \\
$1350,-$ & - & Adelboden, BE
\end{tabular}

\section{Bewertung des Ereignisses}

Kein Erdbeben.

\section{Korrekturvorschläge}

- Verwechslung mit dem Beben von Friaul im Januar 1348

- Verwechslung mit Jülich, D, 1349

- Verwechslung mit dem Bergsturz von 1375

\footnotetext{
15 Lucas et cliccum/Terre motu/Dant tibi notum. Damit ist der Tag des Evangelisten Lukas gemeint, der 18. Oktober, während man das Jahr 1356 durch die Umstellung der Buchstaben des Kunstwortes „CLICCUM“ erkennt: MCCCLVI.

16 Gemäss mündlicher Auskunft von Benjamin Edwards, SED, ETHZ, August 2009.
} 


\section{Überlieferung}

- Hoff (1840-1841)

- Volger (1857)

- Gils, Van \& Leydecker (1991)

- Alexandre (1994)

\section{Interpretation}

Hoff (1840-1841) zitiert eine Arbeit von Schmieder über Geognosie (1802, S. 141), wonach 1350 in der Schweiz ein Erdbeben einen Berg gespaltet haben soll. Dafür sind aber bis heute keine Augenzeugenberichte nachweisbar. Die Lokalisierung „Adelboden“ stammt aus Van Gils \& Leydecker (1991), auch hier fehlt ein entsprechender Quellenverweis.

Momentan kann über die Meldungen nur spekuliert werden. Möglicherweise ist das Erdbeben vom 25. Januar 1348 von Friaul mit seinem Bergsturz gemeint, oder es handelt sich um eine Verwechslung mit dem angeblichen Bergsturz von 1375. Es könnte sich aber auch um eine Verwechslung mit dem Jülich-Beben von 1349 (NordrheinWestfalen, Alexandre 1994) handeln. Bei dieser Quellenlage muss für das Gebiet der Schweiz eine Falschmeldung angenommen werden.

\section{Bemerkungen}

Vgl. im ECOS-09 die Erdbeben vom 25. Januar 1348 im Friaul, I, und den angeblichen Bergsturz von 1375.

\begin{tabular}{|c|c|c|}
\hline $\begin{array}{l}\text { Datum } \\
1356,18 . \text { Oktober }\end{array}$ & $\begin{array}{ll}\text { Zeit (UTC) } \\
-\quad 16 \text { Uhr } \\
\quad \text { (Vorbeben) } \\
-\quad 21 \text { Uhr } \\
\\
\text { (Hauptstoss) }\end{array}$ & $\begin{array}{l}\text { Ort } \\
\text { Basel }\end{array}$ \\
\hline
\end{tabular}

\section{Bewertung des Ereignisses}

Sicheres Ereignis.

\section{Parameter nach ECOS}

- 16 Uhr lx: 7

- 21 Uhr lx: 9

Einige Publikationen zum stärksten Schweizer Erdbeben im letzten Jahrtausend:

- Wackernagel (1856)

- Müller (1956) 
- Mayer-Rosa \& Cadiot (1979)

- Wechsler (1987)

- Meghraoui (2001)

- Becker \& Davenport (2003)

- Lambert (2005)

- Meyer, W. (2006a)

- Meyer, W. (2006b)

- Schwarz-Zanetti (2006b)

- Wild (2006)

- $\quad$ Gisler (2008c)

- Fäh et al. (2009)
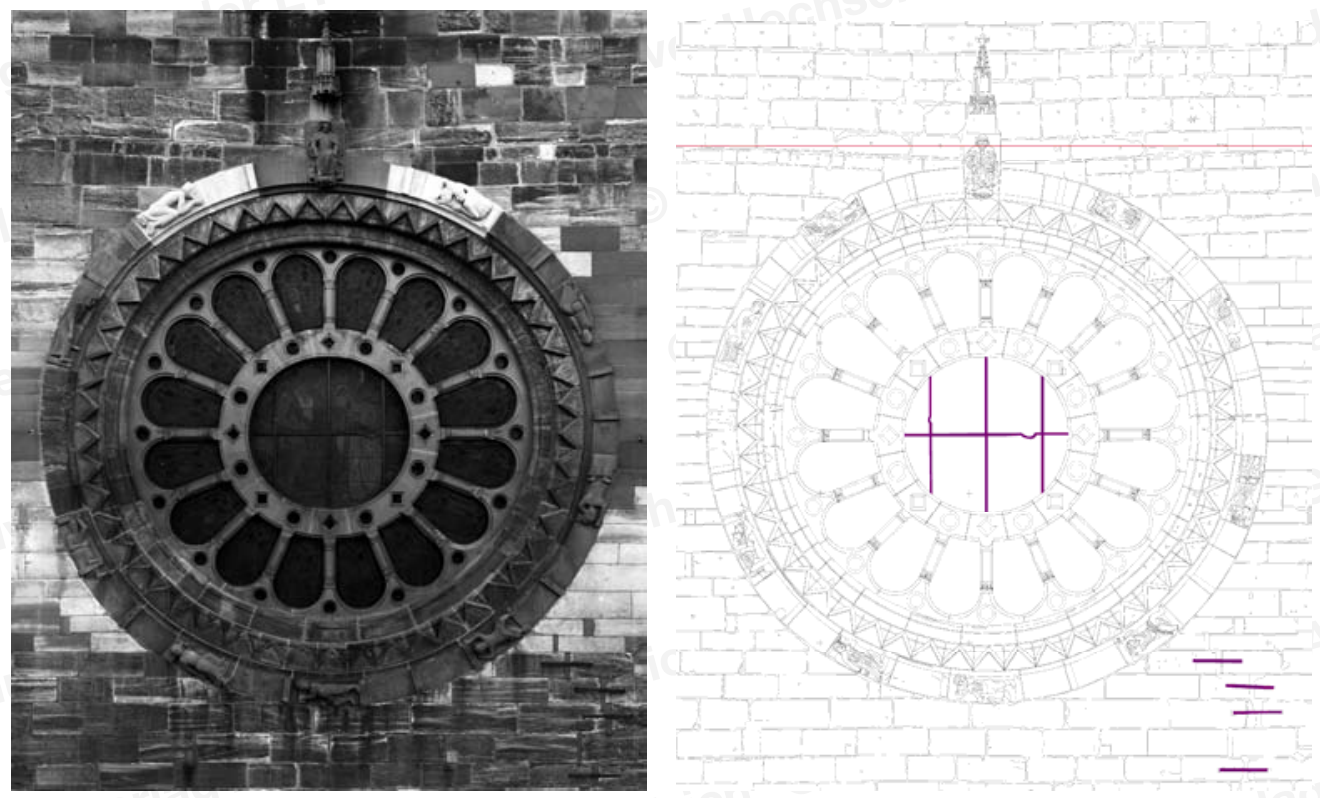

Abbildungen 2 und 3: $\quad$ Erdbebenschäden vom 18. Oktober 1356 an der Glücksrad genannten Fensterrosette des Basler Münsters

Besonders gut sind die Schäden des Erdbebens von 1356 am Nordquerhaus des Basler Münsters zu erkennen: Das Glücksrad aus der Zeit um 1225 wurde deutlich gestaucht, so dass es nicht mehr kreisförmig, sondern leicht oval ist. Die massiven Einwirkungen der Erdstösse lassen sich in dieser Mauerpartie auch am Fugenbild ablesen. Die Metallklammern unterhalb des Glücksrads stammen noch aus der Phase des Wiederaufbaus.

(Planzeichnung: Überarbeitet durch die gbvd, Gesellschaft für Bildverarbeitung, Vermessung und Dokumentation, D-79379 Müllheim, Copyright Münsterbauhütte Basel). 


\section{Makroseismische Parameter}

12 Uhr (UTC):

\begin{tabular}{|l|l|l|l|l|l|l|}
\hline Ortschaft & Länge & Breite & $I_{\min }$ & $I_{\max }$ & $I_{\text {w }}$ & Datenqualität \\
\hline Konstanz & 47.56 & 7.59 & 4 & & & sehr schwach \\
\hline
\end{tabular}

13 Uhr (UTC):

\begin{tabular}{|l|l|l|l|l|l|l|}
\hline Ortschaft & Länge & Breite & $I_{\min }$ & $I_{\max }$ & $I_{w}$ & Datenqualität \\
\hline Konstanz & 47.56 & 7.59 & 3 & & & sehr schwach \\
\hline
\end{tabular}

14 Uhr (UTC):

\begin{tabular}{|l|l|l|l|l|l|l|}
\hline Ortschaft & Länge & Breite & $I_{\min }$ & $I_{\max }$ & $I_{\mathrm{w}}$ & Datenqualität \\
\hline Konstanz, DDD & 47.56 & 7.59 & 3 & & & sehr schwach \\
\hline
\end{tabular}

16 Uhr (UTC):

\begin{tabular}{|l|l|l|l|l|l|l|}
\hline Ortschaft & Länge & Breite & $I_{\min }$ & $I_{\max }$ & $I_{w}$ & Datenqualität \\
\hline Konstanz & 47.67 & 9.18 & 4 & & & sehr schwach \\
\hline Basel & 47.56 & 7.59 & 6 & 8 & 7 & sehr schwach \\
\hline Strasbourg & 48.57 & 7.74 & 5 & 5 & 5 & schwach \\
\hline
\end{tabular}

21 Uhr (UTC):

\begin{tabular}{|c|c|c|c|c|c|c|}
\hline Ortschaft & Länge & Breite & $I_{\min }$ & $I_{\max }$ & $I_{w}$ & Datenqualität \\
\hline Seelisberg & 46.97 & 8.59 & & 6 & & sehr schwach \\
\hline Gelterkinden & 47.46 & 7.86 & 5 & & & sehr schwach \\
\hline Reims & 49.26 & 4.04 & & & verspürt & sehr schwach \\
\hline Engelberg & 46.82 & 8.4 & & & verspürt & sehr schwach \\
\hline Speyer & 49.32 & 8.44 & & & verspürt & sehr schwach \\
\hline Paris & 48.7 & 2.33 & & & verspürt & sehr schwach \\
\hline Montbeliard & 47.52 & 6.8 & & & verspürt & sehr schwach \\
\hline Colmar & 48.09 & 7.37 & & & verspürt & sehr schwach \\
\hline Basel & 47.56 & 7.59 & 8 & 9 & 9 & gut \\
\hline Sissach & 47.46 & 7.81 & 8 & 9 & 9 & sehr schwach \\
\hline Pratteln & 47.52 & 7.69 & 8 & 9 & 9 & sehr schwach \\
\hline Lörrach & 47.62 & 7.67 & 7 & 8 & 8 & sehr schwach \\
\hline Wittnau & 47.48 & 7.98 & 7 & 9 & 8 & schwach \\
\hline Duggingen & 47.45 & 7.61 & 7 & 8 & 8 & sehr schwach \\
\hline
\end{tabular}




\begin{tabular}{|c|c|c|c|c|c|c|}
\hline Ortschaft & Länge & Breite & $I_{\min }$ & $I \max$ & Iw & Datenqualität \\
\hline Muttenz & 47.52 & 7.65 & 7 & 8 & 8 & sehr schwach \\
\hline Blotzheim & 47.6 & 7.5 & 7 & 9 & 8 & schwach \\
\hline Leymen & 47.5 & 7.48 & 7 & 8 & 8 & sehr schwach \\
\hline Kiffis & 47.45 & 7.37 & 7 & 8 & 8 & sehr schwach \\
\hline Buus & 47.51 & 7.86 & 7 & 8 & 8 & sehr schwach \\
\hline Pfeffingen & 47.46 & 7.59 & 7 & 8 & 8 & sehr schwach \\
\hline Oberlarg & 47.47 & 7.23 & 7 & 8 & 8 & sehr schwach \\
\hline Frenkendorf & 47.5 & 7.72 & 7 & 8 & 8 & sehr schwach \\
\hline Bubendorf & 47.45 & 7.74 & 7 & 8 & 8 & sehr schwach \\
\hline Delémont & 47.37 & 7.34 & 7 & 8 & 8 & sehr schwach \\
\hline Bern & 46.95 & 7.44 & 6 & 7 & 7 & schwach \\
\hline Avallon & 47.49 & 3.92 & 6 & 7 & 7 & sehr schwach \\
\hline Oltingue & 47.51 & 7.4 & 6 & 7 & 7 & sehr schwach \\
\hline Dijon & 47.32 & 5.05 & 6 & 7 & 7 & sehr schwach \\
\hline Pleigne & 47.41 & 7.29 & 7 & 8 & 7 & sehr schwach \\
\hline Moutier & 47.28 & 7.37 & 6 & 8 & 7 & sehr schwach \\
\hline Olsberg & 47.52 & 7.78 & 6 & 8 & 7 & sehr schwach \\
\hline Besançon & 47.23 & 6.02 & 6 & 7 & 7 & sehr schwach \\
\hline Kaiseraugst & 47.54 & 7.74 & 6 & 8 & 7 & sehr schwach \\
\hline Montcenis & 46.8 & 4.38 & 6 & 7 & 7 & sehr schwach \\
\hline Montreal & 47.53 & 4.02 & 6 & 7 & 7 & sehr schwach \\
\hline Lantenay & 47.34 & 4.87 & 6 & 7 & 7 & sehr schwach \\
\hline Maisey-le-Duc & 47.85 & 4.68 & 6 & 7 & 7 & sehr schwach \\
\hline Montrond-le-Château & 47.15 & 6.05 & 6 & 7 & 7 & sehr schwach \\
\hline Châtillon & 46.08 & 6.58 & 6 & 7 & 7 & sehr schwach \\
\hline Flavigny-sur-Ozerain & 47.52 & 4.53 & 6 & 7 & 7 & sehr schwach \\
\hline Semur-en-Auxois & 47.51 & 4.33 & 6 & 7 & 7 & sehr schwach \\
\hline Wahlern & 46.82 & 7.35 & 6 & 7 & 7 & sehr schwach \\
\hline Beaune & 47.03 & 4.83 & 6 & 7 & 7 & sehr schwach \\
\hline Boncourt & 47.5 & 7.02 & 7 & 8 & 7 & sehr schwach \\
\hline Montbard & 47.63 & 4.33 & 6 & 7 & 7 & sehr schwach \\
\hline Solothurn & 47.21 & 7.54 & 6 & 8 & 7 & sehr schwach \\
\hline Riehen & 47.58 & 7.66 & 6 & 8 & 7 & sehr schwach \\
\hline
\end{tabular}




\begin{tabular}{|l|l|l|l|l|l|l|}
\hline Ortschaft & Länge & Breite & $I_{\min }$ & $I_{\max }$ & $I_{\text {w }}$ & Datenqualität \\
\hline Strasbourg & 48.57 & 7.74 & 5 & 6 & 6 & schwach \\
\hline Baldegger See & 47.2 & 8.25 & 6 & 7 & 6 & sehr schwach \\
\hline Rheinfelden & 47.55 & 7.79 & 6 & 7 & 6 & sehr schwach \\
\hline Zürich & 47.37 & 8.54 & & 6 & 5 & sehr schwach \\
\hline Konstanz & 47.67 & 9.18 & 4 & 6 & 5 & schwach \\
\hline Vierwaldstättersee & 46.99 & 8.57 & 4 & 6 & 5 & sehr schwach
\end{tabular}

\section{Datum}

1357, 8. Mai
Zeit (UTC)

16 Uhr

\section{Ort}

Strasbourg, F

\section{Bewertung des Ereignisses}

Sicheres Ereignis.

\section{Parameter nach ECOS}

Ix: 7

\section{Überlieferung u. a. in:}

- Gropp (1736)

- Manuskript EAF: Ha 583. Synopsis Annalium Monasterij S. Petri in nigra Silva O.S.B (Mitte 18. Jahrhundert)

- Walch (1950)

- SisFrance (24. August 09), ohne Intensitätsbestimmung

\section{Quellen}

- Diessenhofen (1868): S. 108 [1357]: „Weiter am 5. Mai17 in der Früh kam vor Sonnenaufgang das Erdbeben (wieder), welches am Lukastag des Jahres $56^{18}$ angefangen hatte, jedoch an verschiedenen Orten ununterbrochen gespürt wurde, und am 8. Tag des Mai ${ }^{19}$ zur Vesperstunde ein grosses Erdbeben, ein zweites in der Nacht ${ }^{20}$."

- Closener (1870): „In dem meien an sant Suphien ${ }^{21}$ obent do man zalt 1357 jor, do kam ein ertbideme umbe munstergunplete ${ }^{22} z i t$, der was grosser denne keinre vor-

17 5. Mai 1357.

18 18. Oktober 1356.

19 8. Mai 1357.

20 Ob sich „magnus“ (gross) auf das erste oder zweite Beben bezieht, ist aus der Edition nicht ganz klar ersichtlich; wahrscheinlicher ist vom Satz her das erste.

21 Im Allgemeinen wird die Heilige Sophie am 15. Mai gefeiert, in Strassburg aber am 10. Mai, hier also am Abend zuvor. 
mols gewesen waz, und det ouch merren schaden an glochusern und an zierkemmin denne die vordern hettent geton. (...) “

- Annales Francofurtani (1868): (1357, 5. und 8. Mai) „Im Jahre des Herrn 1357, am 5. Mai zwischen dem Morgenläuten und dem Tagesanbruch, ereignete sich ein grosses Erdbeben. Im selben Jahr 1357, am 8. Mai, direkt nach der Vesper, war ein sehr grosses Erdbeben und danach mehrmals, wird gesagt, ein ähnliches Erdbeben im selben Jahr am 7. August (?), ungefähr um Mitternacht.“

\section{Interpretation}

Mit drei zeitgenössischen Quellen sind mehrere Beben im Raum Strassburg-FrankfurtKonstanz in der ersten Dekade des Mais 1357 sicher nachgewiesen, wobei in Strassburg kleine Schäden entstanden sind. Die Datierung des Hauptbebens muss gemäss der Konstanzer und der Frankfurter Quelle auf den 8. Mai um die Vesper angesetzt werden. Beim Datum 9. Mai in der Closener Chronik, interpretiert als Vorabend des 10. Mais, das auch in SisFrance angegeben wird, handelt es sich vermutlich um einen Irrtum. Auch die in mehreren Kompilationen genannte Datierung „14. Mai“ beruht auf einem Fehler, denn in Strassburg wird, abweichend vom allgemeinen Gebrauch, der Namenstag der Heiligen Sophie bereits am 10. Mai gefeiert.

Interessant an der Closener-Formulierung ist, dass der stärkste Stoss grösser gewesen sei als die vorhergehenden und mehr Schaden angerichtet habe. Der Plural zeigt, dass mit mehr Erdbeben als nur demjenigen vom 5. Mai verglichen wird. Damit stellt sich die Frage, ob hier sogar noch das Erdbeben vom 18. Oktober 1356 gemeint ist.

Diese Schadensbeschreibung für Strassburg wird von der Kompilation aus dem Kloster St. Peter im Schwarzwald (Synopsis Annalium, EAF) und teilweise von der Chronik von Lucelle (Walch, 1950), beide aus dem 18. Jahrhundert, unterstützt. Dabei könnte es sich einerseits um eine Verwechslung mit 1356 handeln, doch andererseits könnte sich tatsächlich ein weiteres Schadenbeben dahinter verstecken, dessen Ausmass bisher unbekannt ist. So fällt auf, dass Gropp in seiner Chronik aus dem Jahr 1763 die Burg Wildenberg in Kirchzell, östlich vom Odenwald, als vom 1356-Beben zerstört bezeichnet; was aber mit einer Entfernung von $250 \mathrm{~km}$ von Basel wenig glaubwürdig ist. Falls es sich dabei nicht um einen Irrtum Gropps handelt, wäre in Zukunft abzuklären, ob vielleicht ein Zusammenhang mit dem 1357-Ereignis besteht.

\section{Exkurs:}

Schwarz-Zanetti (2008c)

\section{Makroseismische Parameter}

\begin{tabular}{|l|l|l|l|l|l|l|}
\hline Ortschaft & Länge & Breite & $I_{\min }$ & $I_{\max }$ & $I_{\text {w }}$ & Datenqualität \\
\hline Strasbourg & 48.57 & 7.74 & 6 & 7 & 7 & sehr schwach \\
\hline Frankfurt am Main & 50.11 & 8.65 & 4 & 5 & 5 & sehr schwach \\
\hline Konstanz & 47.67 & 9.18 & 4 & 5 & 5 & sehr schwach \\
\hline
\end{tabular}

22 Komplet: Abendgebet als Schluss der katholischen kirchlichen Tageszeit, vor Sonnenuntergang. 


\section{Bemerkungen}

Vgl. auch SisFrance.

\begin{tabular}{|c|c|c|}
\hline $\begin{array}{l}\text { Datum } \\
-\quad 1357,9 . \text { Mai } \\
-\quad 1357,14 . \text { Mai }\end{array}$ & $\begin{array}{l}\text { Zeit } \\
- \\
-\end{array}$ & $\begin{array}{l}\text { Ort } \\
-\quad \text { Strasbourg, F } \\
-\quad \text { Strasbourg, F }\end{array}$ \\
\hline
\end{tabular}

\section{Bewertung des Ereignisses}

Keine Erdbeben: Datierungsfehler.

\section{Korrektur}

1357, 8. Mai

\section{Überlieferung u. a. in:}

- Bertrand (1756)

- Volger (1857)

- MECOS-99

\section{Interpretation}

Wie bereits beim Erdbeben vom 8. Mai 1357 aufgeführt, basiert das Datum 14. Mai auf einer Falschinterpretation der Aussage in der Chronik von Fritsche Closner, denn in Strassburg wird das Fest der Heiligen Sophie, abweichend vom sonstigen Gebrauch, bereits am 10. Mai gefeiert (Grotefend, 1991, S. 99). Beim Vorabend handelt es sich eigentlich um den 9. Mai. Da jedoch zwei weitere zeitgenössische Beobachter (Chronik Heinrich von Diessenhofen und Frankfurter Annalen) auf den 8. Mai datieren, ist dies das wahrscheinlichere Datum.

Teilweise publiziert in:

Schwarz-Zanetti (2008c)

\section{Bemerkungen}

Vgl. das Erdbeben vom 8. Mai 1357. 


\begin{tabular}{|c|c|}
\hline $\begin{array}{l}\text { Datum } \\
1358\end{array}$ & Zeit \\
\hline
\end{tabular}

\section{Bewertung des Ereignisses}

Kein Erdbeben.

\section{Korrektur}

Kein Korrekturvorschlag.

\section{Überlieferung u. a. in:}

- Volger (1857): 1358. Erdbeben in Bündten, welches sehr heftig in Chur empfunden wurde (aus „Der neue Sammler“, 1811, Jhg. 6, S. 208). - „Heftiges Erdbeben in der Gegend von Chur.“

- Candreia (1905), S. 26-27: „Anno 1358 ist ein erschröckliches Erdbeben (in Chur) gewesen.“ Churer Chronik (Bündner Volksblatt IV. 195), ohne Quellenangabe, aus den Aufzeichnungen eines Pfarrers entnommen.

- Mecos-99

\section{Interpretation}

Da keine Originalquellen nachweisbar sind, handelt es sich mit grosser Wahrscheinlichkeit um eine Falschmeldung. Es könnte sich auch um eine Verwechslung mit den folgenden Ereignissen handeln:

- 1258 Chur, wahrscheinlichste Variante (vgl. das für 1258 zitierte Nekrologium „Nota Curiensis“ von Chur, MGH Necrologia 1 (1888))

- 1356 Basel

- 1348 Friaul, I (vormals in Villach, A, lokalisiert)

\section{Bemerkungen}

Vgl. das Erdbeben in Chur von 1258.

\section{**}

\begin{tabular}{|l|l|l} 
Datum & Zeit & Ort \\
\hline 1363, 24. Juni & Frühmesse & Nicht lokalisierbar (Strasbourg, F)
\end{tabular}

\section{Bewertung des Ereignisses}

Unsicheres Ereignis.

\section{Parameter nach ECOS}

Ix: verspürt 


\section{Überlieferung u. a. in:}

- Tschamser Chronik von Thann (1864)

- Langenbeck (1892)

- Sieberg (1940)

- $\quad$ Rothé \& Schneider (1962)

- Gils, Van \& Leydecker (1991)

- MECOS-99

\section{Quelle}

Chronik des Jakob Twinger von Königshofen (1871), 1345-1420.

\section{Interpretation}

Es ist die Tschamser Chronik von Thann (1864) im Elsass, die ohne Quellenverweis von einem zerstörenden Beben in den Südvogesen im Jahre 1363 spricht. Langenbeck (1892) übernimmt die Formulierung, auf ihn stützt sich zuerst Sieberg (1940), auf diesen dann Rothé und Schneider (1962).

Mit grosser Wahrscheinlichkeit steht hinter den Kompilationen die zeitgenössische Erdbebennotiz in der Chronik von Twinger von Königshofen (1345-1420), obwohl die kompilatorische Datierung auf den 3. Juli (vgl. unten) nicht erklärbar ist: „24. Juni 1363. Do men zalte 1363 jor zuo sünigihten, do kam ein ertbideme zuo Strosburg umb tagemessezit (ungefähr 6 Uhr).“ Die Datierung mit dem Begriff „sünigiht“, die eigentlich Sonnengang oder Sonnenwende meint, bezieht sich im Mittelalter auf den Johannistag, also den 24. Juni. Schäden sind nur in den Kompilationen erwähnt, nicht aber beim Augenzeugen Königshofen. Da jedoch nach dem 1356-Beben von Basel ein weiteres Schadenbeben in der Chronistik nachweisbar sein sollte, ist bezüglich Schäden eher ein Irrtum anzunehmen. Dies ergibt eine Wertung als „unsicher“ mit der Intensität „verspürt“.

\section{Makroseismische Parameter}

\begin{tabular}{|l|l|l|l|l|l|l|}
\hline Ortschaft & Länge & Breite & $I_{\min }$ & $I_{\max }$ & $I_{w}$ & Datenqualität \\
\hline Strasbourg & 48.57 & 7.74 & & & verspürt & sehr schwach \\
\hline
\end{tabular}

\section{**}

Datum

1363, 3. Juli

\section{Zeit}

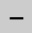

\section{Ort}

Thann, Elsass, F

\section{Bewertung des Ereignisses}

Kein Erdbeben: Datierungsfehler. 


\section{Korrektur}

1363, 24. Juni

\section{Überlieferung u. a. in:}

- Tschamser Chronik von Thann (1864)

- Langenbeck (1892)

- Sieberg (1940)

- $\quad$ Rothé und Schneider (1962)

- Gils, Van \& Leydecker (1991)

- MECOS-99

\section{Interpretation}

Die eigentliche Herkunft dieses Juli-Datums ist unbekannt, es ist zumindest seit der Tschamser Chronik von Thann nachweisbar; die oben aufgeführten Kompilationen beziehen sich aber indirekt auf die Chronik von Jakob Twinger von Königshofen (1871), die eindeutig das Datum 24. Juni 1363 nennt.

\section{Bemerkungen}

Vgl. das Erdbeben vom 24. Juni 1363.

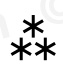

\begin{tabular}{|l|l|l}
\hline Datum & Zeit & Ort \\
\hline 1364, 11. Mai & - & Thann - Haut Rhin, F
\end{tabular}

\section{Bewertung des Ereignisses}

Kein Erdbeben.

\section{Korrektur}

Überlieferung u. a. in:

- Langenbeck (1892)

- Sieberg (1940)

- $\quad$ Rothe \& Schneider (1968)

- MECOS-99

- SisFrance (9. August 2009)

Nicht vorhanden in der zeitgenössischen Quelle:

Chronik des Jakob Twinger von Königshofen (1871) 


\section{Interpretation}

Die Kompilatoren mit diesem Erdbeben stützen sich auf Langenbeck (1892), dieser aber angeblich auf den zeitgenössischen Chronisten Königshofen (1871, S. 864). In dessen von Closener übernommenen und erweiterten Erdbebenchronik ist aber kein Hinweis auf 1364 vorhanden, sodass auf eine Falschmeldung geschlossen werden muss.

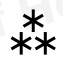

\begin{tabular}{l|l|l}
\hline Datum & Zeit & Ort \\
1 1369, 26. November & - & Mailand, I \\
\hline
\end{tabular}

\section{Bewertung des Ereignisses}

Kein Erdbeben.

\section{Korrektur}

\section{Überlieferung in:}

- Baratta (1901)

- Rüttener (1995)

- MECOS-99

Nicht vorhanden in:

- Volger (1857)

- Candreia (1905)

- Giessberger (1922)

- Boschi (1997)

- Guidoboni (2005b)

- DOM4.1

\section{Interpretation}

Dieses angebliche italienische Erdbeben ist in Schweizer Quellen und Kompilationen nicht nachweisbar. Im MECOS-99 wurde es nur aufgrund von Rüttener aufgenommen, der es seinerseits vermutlich aus der italienischen Kompilation von Baratta (1901, S. 56) übernommen hat. Dieser wiederum stützte sich auf ein unbekanntes Manuskript in einer nicht datierten Arbeit von Mercalli. Darin wird es in Monza (Milano) lokalisiert, wo es Schäden an einigen Häusern angerichtet habe. Da dieses Erdbeben weder in Guidoboni noch in DOM4.1 erwähnt wird, muss es vorläufig als Falschmeldung gewertet werden.

\section{Bemerkungen}

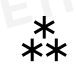




\section{Datum \\ 1372, 1. Juni

\section{Bewertung des Ereignisses}

Unsicheres Ereignis.

\section{Parameter nach ECOS}

Ix: verspürt

\section{Überlieferung u. a. in:}

- Klingenberger Chronik (1861), Mitte 15. Jh. entstanden.

- Luginbühl: Brennwald, Schweizerchronik (1908), entstanden 1508-1516.

- Stumpf (1548): Erwähnt als erster einen kleinen Schaden am Münster.

- Lycosthenes (1557)

- Campell (Plattner, 1887), 1570-1576 entstanden.

- Wurstisen (1580)

- Gross (1624)

- Scheuchzer (1706)

- Volger (1857)

- SisFrance, www.sisfrance.net; enthält weitere bibliographische Verweise.

\section{Quellen}

Einziger zeitgenössischer Nachweis:

Jacob Twinger von Königshofen (1871), S. 864: „Do men zalte 1372 jor, am ersten tage des brochmonates [1. Juni 1372] kam ein ertbideme zuo Strosburg.“

Nicht nachweisbar in folgenden zeitgenössischen Quellen:

- Grosse und Kleine Basler Annalen (Bernoulli, Bd. 5, 1895)

- Chronik. Die älteste deutsche Chronik von Colmar. (1888), hsg. von A. Bernoulli.

\section{Interpretation}

Unerklärlicherweise werden in verschiedenen Kompilationen für die Jahre 1372, 1376, 1378, 1380, 1387 und 1572 ähnliche Erdbebendatierungen aufgeführt, und zwar meist mit der Tagesdatierung 1. Juni, wenige auch mit 1. Juli. Nicht immer werden sie ausdrücklich lokalisiert, am häufigsten jedoch in Basel, wobei dort seit Stumpf (1548) auch Schäden für Basel angeführt werden.

Nachweisbar ist bisher jedoch nur eine zeitgenössische Erdbeben-Beobachtung für den 1. Juni 1372, nämlich für Strassburg in der Chronik von Jakob Twinger von Königshofen, die aber keine Schäden erwähnt. 
Makroseismische Parameter

\begin{tabular}{|l|l|l|l|l|l|l|}
\hline Ortschaft & Länge & Breite & $I_{\min }$ & $I_{\max }$ & $I_{w}$ & Datenqualität \\
\hline Strasbourg, F & 48.58 & 7.75 & & & verspürt & sehr schwach \\
\hline
\end{tabular}

\section{Bemerkungen}

Vgl. die Falschmeldungen im ECOS-09 zu 1372, 1378, 1380 und 1572.

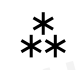

\begin{tabular}{l|l|l} 
Datum & Zeit & Ort \\
-13720601 & - & - Aesch-Basel \\
-13780601 & - & - Glarus \\
-13800701 & - & - Bern \\
-15720601 & - & - Basel
\end{tabular}

\section{Bewertung der Ereignisse}

Keine Erdbeben: Datierungsfehler.

\section{Korrektur}

1372, 1. Juni, in Strassburg

\section{Überlieferung u. a. in:}

- Klingenberg Chronik (1861), Mitte 15. Jh. entstanden, datiert 1372/06/01.

- Brennwald (Luginbühl, 1908), entstanden 1508-1516, datiert 1380/06/01.

- $\quad$ Stumpf (1548), datiert 1372, ohne Tag und Monat.

- $\quad$ Lycosthenes (1557), datiert 1376/06/01 und 1387/06/01.

- Campell in Candreia (1905) datiert 1372/06/01.

- Wurstisen (1580), stützt sich wahrscheinlich auf Stumpf und Brennwald, datiert 1372/06/01.

- Gross (1624) stützt sich wahrscheinlich auf Stumpf, Brennwald und Wurstisen und datiert mit 1372/06/01.

- Klauber (1617) datiert auf 1572/06/01, wahrscheinlich ein Druckfehler.

- Scheuchzer (1706): datiert 1372/06/01, stützt sich auf Stumpf und Wurstisen.

- Bertrand (1756): datiert auf 1372/06/01 und auf den 1372/07/01, letzteres Datum stamme von Buxtorf (Quelle konnte bisher nicht eruiert werden), der auch die Schäden am Münster von Basel erwähnt.

- Guéneau de Montbelliard (1761): datiert 1372/061, 1372/07/01 und stützt sich dabei offensichtlich auf Bertrand; zusätzlich erwähnt er noch 1380/07/01.

- Perrey (1845); datiert 1378/06/01.

- $\quad$ Volger (1857): zitiert 1372/06/01 und 1378 und 1380.

- SisFrance, www.sisfrance.net; enthält weitere bibliographische Verweise, keine Intensität zugeteilt. 
Einziger zeitgenössischer Nachweis:

Chronik des Jacob Twinger von Königshofen (1871), S. 864: „Do men zalte 1372 jor, am ersten tage des brochmonates [1. Juni 1372] kam ein ertbideme zuo Strosburg.“"

Nicht nachweisbar in folgenden zeitgenössischen Quellen:

- Grosse und Kleine Basler Annalen (Bernoulli, Bd. 5, 1895)

- Chronik. Die älteste deutsche Chronik von Colmar. (1888)

\section{Interpretation}

Unerklärlicherweise werden in verschiedenen Kompilationen für die Jahre 1372, 1376, 1378, 1380, 1387 und sogar 1572 ähnliche Erdbebendatierungen aufgeführt, und zwar meist mit der Tagesdatierung 1. Juni, wenige auch mit 1. Juli. (In der Kopfzeile sind nur die bisher in der historischen Seismologie bekannten Daten aufgeführt.) Diese Erdbeben werden nicht immer ausdrücklich lokalisiert, am häufigsten jedoch in Basel, wobei dort seit Stumpf (1548) auch Schäden für Basel angeführt werden. Die Quelle von Stumpfs Schadenmeldung konnte bisher nicht gefunden werden. Die Formulierungen sind aber insgesamt so ähnlich, dass Twinger von Königshofen als gemeinsame Vorlage betrachtet werden muss, wobei aber der Grund für die Häufung der Lese- oder Druckfehler nicht ersichtlich ist.

\section{Bemerkungen}

Vgl. das Erdbeben vom 1. Juni 1372.

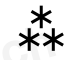

\begin{tabular}{l|l|l} 
Datum & Zeit & Ort \\
\hline 1372, 8. September & - & Aesch-Basel
\end{tabular}

\section{Bewertung des Ereignisses}

Kein Erdbeben: Lokalisierungsfehler.

\section{Korrektur}

Strasbourg, F.

\section{Überlieferung u. a. in:}

- Langenbeck (1882): 7. Sept

- Giessberger (1922)

- Sieberg (1940)

- Rothe \& Schneider (1968)

- Gils, Van \& Leydecker (1991)

- MECOS-99 
Nicht erwähnt in:

- Wurstisen (1580)

- $\quad$ Bertrand (1756)

- Volger (1857)

Zeitgenössische Quelle:

- Chronik des Jakob Twinger von Königshofen, Ende 14. Jh.

\section{Interpretation}

Weder in Wurstisen, Bertrand noch Volger wird dieses September-Erdbeben erwähnt. Erst bei Langenbeck (1892) wird von zwei stark verspürten Erdbeben in Basel und Strassburg im Jahr 1372 geschrieben, nämlich einem Beben im Juni und einem im September, wobei er das zweite jedoch auf den 7. September datierte. Vermutlich wurde aufgrund dieser unklaren Formulierung in späteren Kompilationen auf Basel geschlossen. Van Gils \& Leydecker lokalisieren dieses Beben ohne Begründung in Arlesheim.

Bisher liess sich jedoch nur eine beinah zeitgenössische Notiz bei Jakob Twinger von Königshofen nachweisen, der seine Chronik während seiner Strassburger Zeit verfasst und wahrscheinlich Augenzeuge war, beziehungsweise Strassburger Vorlagen benutzt hat: „Do men zalte 1372 jor, am ersten tage des brochmonates [Juni 1372] kam ein ertbideme zuo Strosburg. Und dernach an unser frowen tage der junger aber ein ertbideme [8. September 1372].“

Somit fand dieses Beben mit einer gewissen Wahrscheinlichkeit eher in Strassburg als in Basel statt. Die Herkunft der Lokalisierung Aesch-Basel lässt sich jedoch nicht mehr eruieren.

\section{Bemerkungen}

Vgl. SisFrance: Vallée du Rhin (Kaiserstuhl?).

\section{***}

\begin{tabular}{|l|l|l}
\hline Datum & Zeit & Ort \\
\hline 1375 & - & Beckenried, NW
\end{tabular}

\section{Bewertung des Ereignisses}

Kein Erdbeben: Bergsturz.

\section{Überlieferung u. a. in:}

- Henne von Sargans, Klingenberg (1861), entstanden Mitte 15. Jh., ältester Nachweis: Text: S. 107, Fussnote: Do man zalt 1374 do kam so vil regen und usbruch der grossen wasser, das in vil landen dörffer und hüsser, vich und lüt under giengent und enweg runnen im wintermanet (November), und kamen erdbidmen und do umb 
liechtmiss [somit ist eher der 2. Februar 1375 gemeint] brachen die berg uff und wurden gross hülinen und giengen $a b$ ir statt uff die felsen, und hatten die lüt gross sorg nacht und tag, Cod. 643 p. 143, Tschudi (Kopie von Klingenberg).

- Sammlung Felix Donat Kyd, Schwyz, 19. Jh. STaSZ, PA 13, Slg, Kyd, Bd. 2, S. 330 oben: Copia aus einem Auszug von Landammann Büntis gesammelten Schriften. Von dem Salzbrunnen. Es war zunächst unter der Kirchen zu Wolfenschiessen ein grosses Dorf genannt Gumligen da war ein Salzbrunnen, welches ein kostlicher Schatz für das Land gewesen, zu dieser Salzpfannen gehörte der Holzhau von Engelberg bis an Sattel im Schwyzerland. Dieses Dorf und Salzbrunnen wurde nachher von einem Stuck Berg verfällt und eingesaaret. [Ohne Jahresangabe.]

- Volger (1857)

- Schaller-Donauer (ca. 1937)

- Montandon (1942-43)

- Gils, Van \& Leydecker (1991)

- MECOS-99

\section{Quellen}

Es sind keine zeitgenösssischen Quellen nachweisbar; weder Stetter von Konstanz, Königshofen von Strassburg, die Chronik der Stadt Zürich noch die Grössern Annalen von Basel erwähnen dieses Beben.

\section{Interpretation}

Der Chronist und Kompilator Klingenberg spricht im 15. Jahrhundert zwar ohne Tagesdatierung und Lokalisierung von Erdbeben im Zeitbereich von 1374-1375, er verbindet den erwähnten Bergsturz aber nicht direkt damit. Umgekehrt fehlt in Donat Kyd bei der Beschreibung des Bergsturzes die Erwähnung eines Erdbebens sogar gänzlich. Die Lokalisierung des angeblichen Erdbebens in Beckenried wiederum stammt aus Van Gils \& Leydecker (1991).

Hingegen sind in der Klimadatenbank Euroclimhist Mittelalter I für das ganze Jahr 1374 und für den Winter 1374-75 erhöhte bis extreme Niederschläge nachgewiesen (Schwarz-Zanetti, 1992), die Bergstürze und Rutschungen begünstigt haben können. Damit kann es sich auch hier um eine Verwechslung der Erschütterung durch Bergstürze und Rutschungen mit einem Erdbeben handeln. Die Lokalisierung Wolfenschiessen bei Kyd konnte nicht weiter gesichert werden.

\section{**}
Datum
Zeit
Ort
1382, 20. April
Col de Balme - Chamonix, F

\section{Bewertung des Ereignisses}

Kein Erdbeben. 


\section{Überlieferung u. a. in:}

- Beuther (1601)

- Bertrand (1756)

- Guéneau de Montbelliard (1761)

- Volger (1857)

- Montandon (1942-43)

- Gils, Van \& Leydecker (1991)

- MECOS-99

- DOM4.1

Nicht vorhanden in:

- Baratta (1901)

- SisFrance (24. August 09)

\section{Quellen}

Bisher sind keine französischen oder schweizerischen Originalquellen nachweisbar.

\section{Interpretation}

Es konnten weder Originalquellen noch italienische oder französische Indizien gefunden werden. Die ältesten kompilatorischen Hinweise stehen in Beuther (1601) und in allgemeiner Form in Bertrand (1756), wobei nirgends Schäden erwähnt werden. Van Gils \& Leydecker (1991) lokalisieren als erste das Beben beim Col de Balme, Chamonix, F, aber ohne Quellenhinweise und bewerten die Intensität mit 6.

Möglicherweise handelt es sich um eine auf Beuther (1601) zurückzuführende irrtümliche Verbindung zweier Beben, denn er führt für den 20. April 1382 ein grosses, sonst unbekanntes französisches Beben auf, basierend auf einer unbekannten Arbeit von Massaeus; ein Datum, das aber z. B. nicht im Katalog von SisFrance aufgeführt ist.

\section{***}

\begin{tabular}{|l|l|l}
\hline Datum & Zeit & Ort \\
\hline 1384, 4. Dezember & - & Thann - Haut Rhin, F
\end{tabular}

\section{Bewertung des Ereignisses}

Keine Erdbeben: Datierungsfehler.

\section{Korrektur}

25. Dezember 1384

\section{Überlieferung u. a. in:}

- Langenbeck (1892)

- Sieberg (1940) 
- Rothé \& Schneider (1968)

- MECOS-99

\section{Interpretation}

Es sind Rothé \& Schneider (1968), die irrtümlicherweise auf den 4. Dezember datieren, was dann von MECOS-99 übernommen worden ist. Die Datierung auf den 24. Dezember kommt auch in hier nicht aufgeführten Kopien vor, doch ist aufgrund der älteren Kompilationen, die den „Stephanstag (26.12.)“ nennen, eher auf den Vorabend des Stephanstags (25.12.) zu schliessen. Es sind keine Schweizer Quellen nachweisbar.

\section{Bemerkungen}

Vgl. das Erdbeben vom 25. Dezember 1384.

\section{***}

\begin{tabular}{|l|l|l} 
Datum & Zeit & Ort \\
1384, 25. Dezember & Nacht & $\begin{array}{l}\text { Nicht lokalisierbar } \\
\text { (Augsburg-Nördlingen) }\end{array}$
\end{tabular}

\section{Bewertung des Ereignisses}

Sehr unsicheres Ereignis, unter Umständen auch Verwechslung mit Gewitter.

\section{Parameter nach ECOS}

Ix: verspürt

\section{Überlieferung}

Ältere Kopien in Chroniken des 15. u. 16. Jh., u. a. in:

- Chronik von Augsburg 1368-1406 (hsg. 1865)

- Chronik des Erhard Wahraus (gest. 1454/55) 1126-1445, Augsburg (1865)

- Chronik des Burkhard Zink (1396 - um 1475): 1368-1468, Augsburg

- Chronik des Hector Mülich 1348-1487, Augsburg

- Müllner (1984) (1565-1634), Annalen der Stadt Nürnberg, 1623, Teil II: 13511469

Jüngere Kompilationen, u. a. in:

- Tschamser (1724)

- Langenbeck (1892)

- Giessberger (1922)

- Sieberg (1940)

- $\quad$ Rothé \& Schneider (1968)

- $\quad$ Gils, Van \& Leydecker (1991); sie datieren auf den 4. Dezember 1384 und lokalisieren in Thann. 
- MECOS-99

- SisFrance (15. Sep. 2009): datiert auf den 24. Dezember

Nicht vorhanden in:

- Bertrand (1756)

- Volger (1857)

- Candreia (1905)

\section{Quellen}

\section{Interpretation}

Im Rahmen des vorliegenden schweizerischen Projektes konnten diese deutschen Erdbebenbeobachtungen bisher nur bis ins 15. Jahrhundert zurückverfolgt werden; vgl. die oben erwähnten Chroniken. Als Beispiel sei hier die Beobachtung aus der Augsburger Chronik von Erhard Wahraus (gest. 1454/55) aufgeführt (1865): „Anno dni. 1384 jaur an sant Stephans nacht in den weichennaechten zwischent liecht kom ein erdbidem und in der nacht kam ein toner und plitzget und toneret." Die auffallende Ähnlichkeit der Augsburger und der Nürnberger Kompilationen ist ein Indiz für eine gemeinsame Vorlage; Schäden werden keine erwähnt.

SisFrance lokalisiert das Beben aufgrund der Kompilatoren Langenbeck, Giessberger, Wolfart und Sieberg im südlich dieses Gebiets gelegenen schwäbischen Memmingen, D. Die Lokalisierung „Thann“ in Van Gils \& Leydecker (1991) wiederum stammt vermutlich aus der Thanner Chronik von Tschamser (1724), ebenso die sonst nicht genannte Datierung „4. Dezember“. In der Kompilation von Giessberger (1922) wiederum sind 16 Belege aus weiteren Kompilationen aufgeführt, die sich meistens auf Nördlingen am 24. Dezember beziehen, wenige nennen auch Memmingen und andere Augsburg, Schwaben und Elsass. In einigen Berichten wird auch der 25. Dezember aufgeführt, zusätzlich wird von Donner und einem Gewitter gesprochen, ein Phänomen, das manchmal mit einem Erdbeben verwechselt wird, vgl. z. B. das Beben am 19. Mai 1523. Der Namenstag des Heiligen Stephan fällt auf den 26. Dezember, somit ist die vorhergehende Nacht gemeint.

Erst zeitgenössische Quellen werden diese Datenlage erhellen können; eine künftige Suche sollte sich vermutlich auf den Raum „Augsburg-Nürnberg-Nördlingen“ konzentrieren.

\section{Makroseismische Parameter}

\begin{tabular}{|l|l|l|l|l|l|l|}
\hline Ortschaft & Länge & Breite & $I_{\min }$ & $I_{\max }$ & $I_{\text {w }}$ & Datenqualität \\
\hline Noerdlingen & 48.85 & 10.5 & & & verspürt & sehr schwach \\
\hline Augsburg & 48.37 & 10.9 & & & verspürt & sehr schwach \\
\hline
\end{tabular}




\begin{tabular}{l|l|l}
\hline Datum & Zeit & Ort \\
$-1391,22$. März & - & - Sundgau, F \\
$-\quad 1394$, 22. März & - & - Brig, VS
\end{tabular}

\section{Bewertung des Ereignisses}

Keine Erdbeben: Datierungsfehler.

\section{Korrektur}

1394, 22. April

\section{Überlieferung}

- Dierauer (1890): Chronik der Stadt Zürich

- Abschriften der Zürcher Chroniken, vgl. Gamper (1984)

- Lycosthenes (1557)

- Ziegler (1674)

- Scheuchzer/Sulzer (1746)

- Bertrand (1756)

- Volger (1857), zitiert Hoff (1840-1841), Beuther (1601) u.a.

- Langenbeck (1892)

- $\quad$ Sieberg (1940)

- Montandon (1942-43), S. 65, vergleicht es nur mit Brig 1755.

- Rothé und Schneider (1968)

- $\quad$ MECOS-99, lokalisiert mit Brig und führt dafür Montandon als Beleg an.

\section{Interpretation}

Die Erdbebenbeschreibungen für die Jahre 1391 und 1394 basieren auf der Chronik der Stadt Zürich: „A. d. 1394 an der mitwuchen in der osterwuchen, die was der 22. tag merzen, do kam ain gross erdbidem zu mittem tag, und kam enkain schad davon in disem land." (Dierauer, 1890, 165) Weil der 22. März im Jahr 1394 kein Mittwoch war, enthält diese Doppeldatierung offensichtlich einen Fehler.

Das Original der Chronik ist nicht überliefert, die Abschriften, die aus dem 15. Jahrhundert und später stammen, enthalten alle die Monatsbezeichnung März, die aber bereits teilweise von zweiter Hand richtigerweise mit April korrigiert worden ist. Es war dann wahrscheinlich Lycosthenes (1557), der als erster fälschlicherweise das Jahr auf 1391 uminterpretierte, da der 22. März auf einen Mittwoch fiel, allerdings in der Woche vor dem Osterfest, das am 26. März stattfand. Die Osterwoche ist jedoch definitionsgemäss die Woche nach Ostern. Im Jahre 1394 fiel Ostern auf den 19. April, der 22. April damit, wie in der Chronik angegeben, auf den Mittwoch nach Ostern.

Da nun mit der Korrektur des Monats nur noch ein einziger Fehler in den Chronikabschriften angenommen werden muss, so ist die Datierung 22. April 1394 die wahrscheinlichere Lösung als diejenige von Lycosthenes mit 22. März 1391. 
Die Lokalisierung „Sundgau“ stammt aus Sieberg (1940), der irrtümlich angibt, sich auf Volger (1857) und Langenbeck (1892) zu stützen. Schliesslich war es Montandon (1942-43, S. 65), der, obwohl „les détails sur les dommages manquent“, eine Parallele zu den grossen Erdbeben in den Jahren 1755 und 1855 im Wallis zog. Daraus schloss der MECOS-99 irrtümlich auf eine Intensität 7 in Brig.

\section{Bemerkungen}

Vgl. das Erdbeben vom 22. April 1394 in Zürich

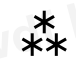

\begin{tabular}{|l|l|l}
\hline Datum & Zeit (UTC) & Ort \\
1394, 22. April & 11 Uhr & $\begin{array}{l}\text { Nicht lokalisierbar } \\
\text { (Zürich) }\end{array}$
\end{tabular}

\section{Bewertung des Ereignisses}

Unsicheres Ereignis.

\section{Parameter nach ECOS}

Ix: 5

\section{Überlieferung}

Abschriften der Zürcher Chronik, ab Mitte des 15. Jh., die Manuskripte sind in Gamper (1984) aufgeführt, darin v. a. die Abschrift der Zürcher Chronik in Jakob Twinger von Königshofen, Stiftsbibliothek St. Gallen, Codex 631; wahrscheinlich aus dem Jahr 1473.

März-Datierung:

- Bertrand (1756)

- Volger (1857)

- Montandon (1942-43)

- MECOS-99

\section{Quellen}

Bisher wurden keine Originalquellen gefunden.

\section{Interpretation}

Die Erdbebenbeschreibung für das Jahr 1394 basiert auf einer Aussage in der Chronik der Stadt Zürich: „A. d. 1394 an der mitwuchen in der osterwuchen, die was der 22. tag merzen, do kam ain gross erdbidem zu mittem tag, und kam enkain [kein] schad davon in disem land." (Dierauer, 1890, S. 165). Es wird ausdrücklich erwähnt, dass es keine Schäden gegeben habe. 
Doch die Doppeldatierung enthält offensichtlich einen Fehler bei der Monatsbezeichnung.

Das Original der Zürcher Chronik ist nicht überliefert, und die Abschriften, die aus dem 15. Jahrhundert und später stammen, enthalten meistens die Monatsbezeichnung März, die aber bereits teilweise von fremder Hand richtigerweise mit April korrigiert worden sind. Denn Ostern fand im Jahre 1394 am 19. April statt, bzw. der Mittwoch danach war tatsächlich der 22. April. Der Zürcher Chronik-Anhang in einem Manuskript von Jakob Twinger von Königshofen in Strassburg, nun in der Stiftsbibliothek St. Gallen (Cod. 631), schreibt beispielsweise schon ausdrücklich „aprillen“. Wahrscheinlich von Lycosthenes (1557) wurde diese Osterdatierung auch mit der Karwoche 1391 verwechselt, wo der Mittwoch auf den 22. März gefallen war.

Da bisher keine weiteren Originalbeobachtungen gefunden worden sind, muss das Ereignis als „unsicher“ gewertet werden.

\section{Makroseismische Parameter}

\begin{tabular}{|l|l|l|l|l|l|l|}
\hline Ortschaft & Länge & Breite & $I_{\min }$ & $I_{\max }$ & $I_{\text {w }}$ & Datenqualität \\
\hline Zürich & 47.37 & 8.54 & 4 & 5 & 5 & sehr schwach \\
\hline
\end{tabular}

\section{Bemerkungen}

Vgl. die Falschmeldungen vom 22. März 1391 und 22. März 1394

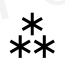

\begin{tabular}{l|l|l} 
Datum & Zeit & Ort \\
-1396, 26. Dezember & - & Bergamo, I \\
-1397, 26. Dezember & -2 &
\end{tabular}

\section{Bewertung des Ereignisses}

Keine Erdbeben: Datierungsfehler.

\section{Korrektur}

1396, 26. November, Monza, I

\section{Überlieferung u. a. in:}

- $\quad$ Baratta (1901): 1397

- DOM4.1: 1396

- Rüttener (1995): 1396 und 1397

- MECOS-99: 1396 und 1397

- Guidoboni et al. (2005b): 26. November 1396

Nicht vorhanden in:

- Candreia (1905)

- Volger (1857) 


\section{Interpretation}

Dieses Erdbeben ist bisher für die Schweiz nicht nachweisbar. Baratta (1901) seinerseits stützt sich auf mehrere Quellen, die hier nicht überprüft werden konnten, und datiert auf das Jahr 1397. Guidoboni (2005b, S. 538-39) datiert dieses Erdbeben aber auf den 26. November 1396 in Monza, Norditalien, und schätzt die Intensität auf 7-8, was für den ECOS-09 übernommen worden ist.

\section{Bemerkungen}

Vgl. im ECOS-09 das Erdbeben vom 26. November 1397.

\begin{tabular}{|c|c|c|}
\hline $\begin{array}{l}\text { Datum } \\
1400\end{array}$ & Zeit & $\begin{array}{l}\text { Ort } \\
\text { Kreuzlingen, TG -Bodenseegebiet }\end{array}$ \\
\hline
\end{tabular}

\section{Bewertung des Ereignisses}

Kein Erdbeben.

\section{Korrektur}

Kein Korrekturvorschlag.

\section{Überlieferung}

- Lersch, Manuskript im GFZ (1897), zitiert in Sieberg (1940)

- Sieberg (1940)

- Gils, Van \& Leydecker (1991)

- MECOS-99

Nicht vorhanden in zeitgenössischem Wettertagebuch:

UBB Manuskript: F. III.8. Wettertagebuch des Basler(?) Anonymus, 1399-1405, Transkription in EuroClimHist Mittelalter Mitteleuropa 1, Schwarz-Zanetti (1992). Zusammenfassende englische Teiltranskription in Thorndike (1949 und 1966).

\section{Interpretation}

Van Gils \& Leydecker (1991) stützen sich auf Sieberg (1940), dieser wiederum auf die handschriftliche Erdbebenchronik von Lersch (GFZ, 1897), die nicht eingesehen wurde. Ihr zufolge habe in Süddeutschland ein grosses Erdbeben stattgefunden, vermutlich im Bodenseegebiet. Van Gils \& Leydecker lokalisieren diesen Bericht in Kreuzlingen. Ein Originalbericht liess sich dazu aber nicht finden, auch nicht im für 1400 lückenlosen Wettertagebuch des sogenannten Basler(?) Anonymus 1399-1405, obwohl dieser auch für Erdbebenbeobachtungen sensibilisiert ist, wie die zwei Beobachtungen vom 14. April 1402 und 23. November 1403 belegen. Möglicherweise handelt es sich um eine zah- 
lenmystische Spekulation anlässlich der runden Jahreszahl, wie bei den Jahren 1000 und 1500.

Aufgrund der sehr unsicheren Quellenlage ist mit grosser Wahrscheinlichkeit auf eine Falschmeldung zu schliessen.

\section{$\stackrel{*}{* *}$}

\begin{tabular}{|l|l|l}
\hline Datum & Zeit & Ort \\
\hline 1402, 14. April & - & Nicht lokalisierbar (Basel)
\end{tabular}

\section{Bewertung des Ereignisses}

Unsicheres Ereignis.

\section{Parameter nach ECOS}

Ix: verspürt

\section{Überlieferung}

Neuer Fund - nicht in den bisherigen Kompilationen vorhanden.

\section{Quelle}

UBB: Ms. Basel F. III. 8. Basler(?) Anonymus, 1399-1405, Lateinisch-deutsche Arbeitsedition in EuroClimHist Mittelalter Mitteleuropa 1, Schwarz-Zanetti (1992). Zusammenfassende englische Teiltranskription in Thorndike (1949 und 1966).

Text:

1402.04.14: Terraemotus [Erdbeben].

\section{Interpretation}

Der anonyme Verfasser des Manuskripts hat im Jahr 1402 das tägliche Wettergeschehen, vermutlich in Basel, lückenlos festgehalten. Seine allgemeine Zuverlässigkeit konnte auch im Vergleich mit weiteren Quellen erhärtet werden. Damit darf auch auf eine grosse Zuverlässigkeit dieser Erdbebenbeobachtung, die nur das Datum und den Begriff „terrae motus“ umfasst, also auch keine Schäden erwähnt, geschlossen werden. Wegen der bis jetzt fehlenden Bestätigung durch eine weitere Quelle kann dieses Ereignis jedoch nur mit „unsicher“ gewertet werden.

Makroseismische Parameter

\begin{tabular}{|l|l|l|l|l|l|l|}
\hline Ortschaft & Länge & Breite & $I_{\min }$ & $I_{\max }$ & $I_{\text {w }}$ & Datenqualität \\
\hline Basel & 47.56 & 7.59 & & & verspürt & sehr schwach \\
\hline
\end{tabular}




\section{Datum \\ Zeit \\ Ort \\ 1403, 23. November \\ Nachts \\ Nicht lokalisierbar (Basel)}

\section{Bewertung des Ereignisses}

Unsicheres Ereignis.

\section{Parameter nach ECOS}

Ix: verspürt

\section{Überlieferung}

Neuer Fund - nicht in den bisherigen Kompilationen enthalten.

\section{Quelle}

UBB: Ms. Basel F. III.8. Basler(?) Anonymus, 1399-1405, Lateinisch-deutsche Arbeitsedition in Schwarz-Zanetti (1992). Zusammenfassende englische Teiltranskription in Thorndike (1949 und 1966).

\section{Text:}

1403.11.23: In hac nocte fuit terremotus. [In dieser Nacht ereignete sich ein Erdbeben.]

\section{Interpretation}

Der anonyme Verfasser dieses Manuskripts hat mit grosser Akribie das tägliche Wettergeschehen verfolgt, was auch durch Quervergleiche mit weiteren Quellen erhärtet werden konnte, Ort der Beobachtungen war vermutlich Basel. Damit darf auch auf eine grosse Zuverlässigkeit seiner Erdbebenbeobachtung, die übrigens keine Schäden erwähnt, geschlossen werden. Wegen der bis jetzt fehlenden Bestätigung durch eine weitere Quelle kann dieses Ereignis jedoch nur mit „unsicher“ bewertet werden.

Makroseismische Parameter

\begin{tabular}{|l|l|l|l|l|l|l|}
\hline Ortschaft & Länge & Breite & $I_{\min }$ & $I_{\max }$ & $I_{\text {w }}$ & Datenqualität \\
\hline Basel & 47.56 & 7.59 & & & verspürt & sehr schwach \\
\hline
\end{tabular}

\section{*}

Datum

1415, 21. Juni

\section{Zeit}

$-$
Ort

Basel

\section{Bewertung des Ereignisses}

Kein Erdbeben: Datierungsfehler 


\section{Korrektur}

1416, 21. Juli

\section{Überlieferung $\mathbf{u}$. a. in:}

- Bertrand (1756)

- Guéneau de Montbelliard (1761)

- Hoff (1840-1841)

- Volger (1856)

- MECOS-99

\section{Zeitgenössische Quelle:}

Bernoulli (1895): „Die Grösseren Basler Annalen 238-1416. Kurze Fortsetzung der Grösseren Basler Annalen: Vermischte Aufzeichnungen auf Blatt 432b.“

\section{Interpretation}

In den zeitgenössischen Quellen lässt sich kein Erdbeben am 21. Juni 1415 nachweisen; dieses angebliche Ereignis ist erst in Bertrands Kompilation (1756) zu finden. Von Hoff (1840-1841) und Volger (1857) berufen sich zwar auf Bertrand, datieren aber irrtümlich auf den 24. Juni 1415. Doch die Formulierung und die Datierung der Beschreibung gleichen der zeitgenössischen Beschreibung des Bebens vom 21. Juli 1416 aus den Grösseren Basler Annalen (Bernoulli 1895), wo auch von einer Flucht aus den Häusern die Rede ist. Deshalb kann bei den 1415-Datierungen mit grösster Wahrscheinlichkeit von einem Lese- oder Druckfehler ausgegangen und auf den 21. Juli 1416 umdatiert werden.

\section{Bemerkungen}

Vgl. das Erdbeben vom 21. Juli 1416.

\section{**}

\begin{tabular}{|l|l|l} 
Datum & Zeit (UTC) & Ort \\
\hline 1416, 21. Juli & $14 \mathrm{Uhr}$ & Nicht lokalisierbar (Basel)
\end{tabular}

\section{Bewertung des Ereignisses}

Sicheres Ereignis.

\section{Parameter nach ECOS}

Ix: 6 


\section{Überlieferung u. a. in:}

- Bernoulli (1902): Die Anonyme Chronik bei Schnitt, sammt Fortsetzung 1495-1541, S. 226: (301) „Anno 1416 an sant Marien Madalennen abent was zuo Basel ein erdbidem, so grosz das die leut usz der stat uff das feld luffen; und thett man grosse krutzgeng."

- $\quad$ Stumpf (1548)

- Scheuchzer (1716/1746)

- Bertrand (1756)

\section{Quellen}

- Bernoulli (1895, S. 44): Die Grösseren Basler Annalen 238-1416. Kurze Fortsetzung der Grösseren Basler Annalen: Vermischte Aufzeichnungen auf Blatt 432b: „1416 Juli 21: Anno domini 1416 in vigilia Magdalene fuit Basilee terremotus major quam in 60 annis evenerat, ita quod homines fugiebant in ortos et suburbia et horrea; et postea habita processio ab episcopo et clero devotissima." [Im Jahre 1416, am Vorabend der Heiligen Magdalena (22. Juli), 21. Juli, ist in Basel das grösste Erdbeben seit 60 Jahren gewesen, derart, dass die Menschen in die Gärten, Vorstädte und Scheunen flohen, danach wurde vom Bischof und dem Klerus eine fromme Prozession veranstaltet.]

- Bernoulli (1895, S. 319-320): Die Chronik Henmann Offenburgs (1379-1459) 1413-1445: „Anno domini 1416, als die glock 3 schluog, uff zinstag nach sant Margethen tag (21. Juli), kam ein gar grosser erpidem und zwen klein; dett doch keinen schaden, und worend all 3 uff obgenanthe stund." Henmann Offenburg lebte ebenfalls in Basel.

\section{Interpretation}

Mit nur zwei zeitgenössischen Quellen und einer Abschrift kann dieses Erdbeben mit wahrscheinlich drei Stössen als knapp gesichert gelten. Zwar werden in den Grösseren Basler Annalen keine Schäden erwähnt, beziehungsweise von Henmann von Offenburg ausdrücklich verneint. Doch eine Panik brach aus und später wurde eine Prozession veranstaltet. In den Kompilationen werden zusätzlich noch die Daten 21. und 24. Juni 1415 und 22. Juli 1416 zitiert, die jedoch nicht in Originalquellen zu finden sind und deshalb mit grösster Wahrscheinlichkeit als Datierungsfehler für den 21. Juli 1416 zu werten sind. Wegen der Panik und der nachfolgenden Prozession wird mit einer Intensität 6 gewertet.

\section{Makroseismische Parameter}

\begin{tabular}{|l|l|l|l|l|l|l|}
\hline Ortschaft & Länge & Breite & $I_{\min }$ & $I_{\max }$ & $I_{w}$ & Datenqualität \\
\hline Basel & 47.56 & 7.59 & 5 & 6 & 6 & mässig \\
\hline
\end{tabular}




\begin{tabular}{|l|l|l|}
\hline Datum & Zeit & Ort \\
\hline 1416, 22. Juli & - & Basel \\
\hline
\end{tabular}

\section{Bewertung des Ereignisses}

Kein Erdbeben: Datierungsfehler

\section{Korrektur}

1416, 21. Juli

\section{Überlieferung u. a. in:}

- Lycosthenes (1557)

- Bertrand (1756)

- Hoff (1840-1841)

- Perrey, Rhin (1845)

- $\quad$ Volger (1857)

- MECOS-99

Zeitgenössische Quelle:

Bernoulli (1895): Die Grösseren Basler Annalen 238-1416

\section{Interpretation}

Vermutlich stammt das Problem aus der fehlerhaften Übersetzung des Begriffes „in vigilia Magdalene“ [also „am Vorabend des Festes der Heiligen Magdalena“ (22. Juli)] aus den Grösseren Basler Annalen (Bernoulli 1895) durch Bertrand (1756). Somit ist der 21. Juli 1416 gemeint. Was aber auch bereits in den Kompilationen des 19. Jahrhunderts vermutet worden ist.

\section{Bemerkungen}

Vgl. 21. Juli 1416.

\section{**}

Datum

1417

\section{Zeit}

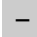

Ort

Neuchâtel

\section{Bewertung des Ereignisses}

Kein Erdbeben. 


\section{Korrektur}

Kein Korrekturvorschlag.

\section{Überlieferung}

- Kopp (1861)

- Tribolet (1876)

- MECOS-99

\section{Interpretation}

Für ein Erdbeben an diesem Datum sind bisher keine Originalquellen nachweisbar. Es gibt auch keinen Hinweis darauf, ob es sich um eine Verwechslung mit dem Basler Schadenbeben vom 12. Dezember 1428 handeln könnte. Die Kurzkompilation von Kopp, die im Bulletin de la Société des Sciences Naturelles de Neuchâtel erschienen ist, führt nur Jahreszahlen auf, ohne irgendwelche Quellenverweise zu geben, und ist somit nicht überprüfbar. Möglicherweise ist die Datierung „1407“ in Tribolet (1876) ein Druckfehler derselben Information. Derartige Datensituationen sind als Falschmeldung zu werten.

\section{$\stackrel{*}{* *}$}

\section{Datum}

1428, 12. Dezember
Zeit

(Abends)

\section{Ort}

Nicht lokalisierbar (Basel)

\section{Bewertung des Ereignisses}

Unsicher.

\section{Parameter nach ECOS}

Ix: 7

\section{Überlieferung}

- Stumpf (1548)

- Bertrand (1756)

- Volger (1857)

- Sieberg (1940)

- Montandon (1942-43)

Nicht vorhanden in:

Zürcher Chroniken, vgl. Gamper (1984). 


\section{Quellen}

- Bernoulli, A. (1890, S. 436): Appenwiler Zusatz: „Des von Ramstein kampf zuo Basel. Anno domini 1428 jor Lucie [13. Dezember] ( Fussnote: Der Zweikampf Johanns von Merlo fand am 12. Dec. 1428 statt; s. oben Rathsb. S. 41.) kam ein froemder ritter gon Basel ... . Noch dem vechten, zem nachtmol, kamend grosz erbidmen, das man reit durch die stat, gebot alle fur zuo loeschen, by lip und guot. do fielend die ziegel ab den techren."

- Bernoulli, A., (1902, S. 304): Die Spätern Aufzeichnungen bei Schnitt 1400-1487: „Anno $1428 \mathrm{kam}$ ein grosser erdbidem zuo Basel, das die ziegel ab den decheren fielent ... und fil kemmy zerbrachen. Und fiel dasz crutz im munster uff sant Gallen thur herab und geschach den Barfuszeren groszer schaden.“

- $\quad$ Ruppert (1891, S. 132) stützt sich auf Dacher (ca. 1425-1470): Des selben jars (1427!) was ain gross erdbidem jn gantzem land vnd weret lang vn(d) verdarbt land und lüt. (Vgl. auch Neuedition von Dacher durch Sandra Wolff, 2008.)

\section{Interpretation}

Die zwei unabhängigen Basler Beobachtungen lassen mit grosser Wahrscheinlichkeit auf ein Schadenbeben im Jahr 1428 schliessen, gemäss dem Quellen-Editor fand es aber schon am 12. Dezember, nicht erst am 13. Dezember, statt. In beiden Quellen werden herabgefallene Ziegel erwähnt, in einer auch Kamine und ein Kreuz vom Münster.

Im Weiteren wäre noch eine Konstanzer Notiz über ein schädliches Erdbeben im Jahr 1427 zu verifizieren, die der Chronist Dacher um 1470 aus einer unbekannten Quelle kopiert hatte. Aufgrund des zeitlich nahen Datums und der Schäden könnte es sich unter Umständen um einen Datierungsfehler für 1428 handeln.

\section{Makroseismische Parameter}

\begin{tabular}{|l|l|l|l|l|l|l|}
\hline Ortschaft & Länge & Breite & $I_{\min }$ & $I_{\max }$ & $I_{w}$ & Datenqualität \\
\hline Basel & 47.56 & 7.59 & 6 & 7 & 7 & schwach \\
\hline
\end{tabular}

\section{***}

\section{Datum}

1428, 13. Dezember

\section{Zeit}

\section{Ort}

Basel

\section{Bewertung des Ereignisses}

Kein Erdbeben: Datierungsfehler.

\section{Korrektur}

1428, 12. Dezember 


\section{Überlieferung}

- Lycosthenes (1557)

- Bertrand (1756)

- Volger (1857)

- Sieberg (1940)

- Montandon (1942-43)

- MECOS-99

\section{Quelle}

Bernoulli, A. (1890): Appenwiler Zusatz.

\section{Interpretation}

Bernoulli (1890, S. 436) verwirft die Chronikdatierung „13. Dezember“ und fixiert das Erdbeben im Zusatz zu Appenwiler aufgrund des am gleichen Tage stattfindenden Zweikampfes auf den 12. Dezember 1428.

\section{Bemerkungen}

Vgl. 12. Dezember 1428

\begin{tabular}{|c|c|c|}
\hline $\begin{array}{l}\text { Datum } \\
\text { 1470, 6. Februar }\end{array}$ & $\begin{array}{l}\text { Zeit (UTC) } \\
4 \text { Uhr }\end{array}$ & $\begin{array}{l}\text { Ort } \\
\text { Aesch-Basel }\end{array}$ \\
\hline
\end{tabular}

\section{Bewertung des Ereignisses}

Kein Erdbeben: Datierungsfehler

\section{Korrektur}

1470, 16. Februar in Basel

\section{Überlieferung u. a. in:}

- Bertrand (1756)

- Volger (1857)

- Sieberg (1940)

- MECOS-99

Zeitgenössische Quellen mit dem Datum 16. Februar 1470:

- Bernoulli (1890): Die Chronik Erhards von Appenwiler 1439-1471, mit ihren Fortsetzungen 1472-1474.

- Bernoulli (1902): Die Grösseren Basler Annalen nach Schnitts Handschrift.

- Bernoulli (1902): Die Chronik in Ludwig Kilchmanns Schuldbuch 1468-1518. 
Falsche Datierung: 16. April 1470:

Bernoulli (1915): Des Kaplans Niklaus Gerung genannt Blauenstein Fortsetzung: „Anno 1470 die 16. Aprilis fuit terremotus omni sine dampno." [Im Jahre 1470, am 16. April war ein Erdbeben ganz ohne Schaden.] Diese April-Datierung wurde aber bereits von Bernoulli aufgrund der obigen drei Quellen auf den 16. Februar korrigiert.

\section{Interpretation}

Bertrand enthält den ältesten Hinweis auf den 6. Februar 1470, was ein Druckfehler sein dürfte. Denn drei zeitgenössische Quellen datieren auf den 16. Februar, eine vierte auf den 16. April. Das Erdbeben wird in den ersten zwei Quellen als "gross“ umschrieben, aber Gerung sagt ausdrücklich „ohne Schaden“, somit ist es als „verspürt“ zu bewerten. Das Erdbeben vom 16. Februar ist in Basel zu lokalisieren.

\section{Bemerkungen}

Vgl. im ECOS-09 das Erdbeben vom 16. Februar 1470.

\begin{tabular}{|c|c|c|}
\hline $\begin{array}{l}\text { Datum } \\
\text { 1471, Mai }\end{array}$ & Zeit & $\begin{array}{l}\text { Ort } \\
\text { Nördlingen, D }\end{array}$ \\
\hline
\end{tabular}

\section{Bewertung des Ereignisses}

Kein Erdbeben.

\section{Korrektur}

\section{Überlieferung}

- Guggenbühl (Wolf, 1856/57): „1471: gwaltige Erdbidem ... .“

- Giessberger (1922): „1471 (ohne Datum): Zu Brichsen [Breisach] ist ein gross Erdbeben gewesen/doch ohne Schaden abgangen (M. Bernhertz $(1616,89))^{\text {“. }}$.

- Sieberg (1940): „1471, Montag vor Christi Himmelfahrt (20.5.). Ein Schadenbeben im Ries, das in Nördlingen Kamine abwarf und den Turm der Pfarrkirche kräftig beschädigte. (Ohne Quellenangabe.)“

- Leydecker (1986)

- Grünthal (2001)

- Erdbebenkatalog Bayerns (http://www.erdbeben-in-bayern.de/erdbebenkatalog/ lokalbeben)

\section{Quellen}

Keine zeitgenössischen Quellen 


\section{Interpretation}

Im Bayerischen Erdbebenkatalog wird dieses Ereignis mit der Intensität 7 bewertet (13. April 2010). Da aber zeitgenössische Nachrichten fehlen, sind diese kompilatorischen Meldungen mit grösster Vorsicht zu verwenden. Grünthal (2001) schliesst ein Erdbeben aus und hält statische Probleme am Turm für wahrscheinlicher. Solange keine zeitgenössischen Quellen gefunden sind, muss dieses Erdbeben deshalb als Falschmeldung gewertet werden.

Verwechslungen mit Stürmen sind ebenfalls kein seltenes Phänomen; zum Beispiel für den 26. Juni 1517 ist in Nördlingen eine derartige Verwechslung mit Hilfe von süddeutschen Wettertagebüchern belegbar, nicht aber für den Mai 1471 (Schwarz-Zanetti 1992).

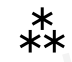

\begin{tabular}{l|l|l} 
Datum & Zeit (UTC) & Ort \\
1474, 11. Dezember & $14 \mathrm{Uhr}$ & Basel
\end{tabular}

\section{Bewertung des Ereignisses}

Kein Erdbeben: Datierungsfehler.

\section{Korrektur}

11. Dezember 1473

\section{Überlieferung}

Neuer Fund, nicht vorhanden in den Kompilationen von:

- Merian (1834)

- Volger (1857)

- Candreia (1905)

- Giessberger (1922)

- MECOS-99

\section{Quelle}

Vischer, W., Boss, H. (Hrsg.), 1880: Johannis Knebel Capellani Ecclesiae Basiliensis Diarium: „Die sabbati 11. mensis decembris [1473] in medio hore tercie et quarte ante vesperas venit terre motus, sed modicus.“ [Am Samstag, 11. Dezember 1473, zwischen drei und vier, vor der Vesper, kam ein Erdbeben, aber mässig.]

\section{Interpretation}

Das Erdbeben vom 11. Dezember 1473 ist bisher nur aufgrund des Tagebuches von Johannes Knebel aus Basel nachgewiesen, worin es ausdrücklich mit „modicus“, also mässig oder gering, qualifiziert wird. Damit wird es kaum eine Intensität über 4 erreicht haben. Die Jahresdatierung ist unklar, muss sich aber aufgrund der Tagesbezeichnung 
"Samstag“ auf das Jahr 1473 beziehen und nicht auf 1474. Da die Wetterbeobachtungen in Knebels Tagebuch sich als sehr zuverlässig erwiesen haben (Schwarz-Zanetti, 1992), darf diese einzelne Erdbebenbeobachtung für 1473 doch mit „unsicher“ bewertet werden.

\section{Bemerkung}

Vgl. im ECOS-99 das Erdbeben vom 11. Dezember 1473.

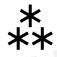

\begin{tabular}{l|l|l} 
Datum & Zeit & Ort \\
1490 & - & Lugano
\end{tabular}

\section{Bewertung des Ereignisses}

Kein Erdbeben.

\section{Korrektur}

\section{Überlieferung u. a. in:}

- Journal de Genève (1855)

- Volger (1857)

- $\quad$ [Candreia (1905)]

- Gils, Van \& Leydecker (1991)

- MECOS-99

- SisFrance (März 2010)

\section{Interpretation}

Das Erdbebendatum 1490 ist mindestens seit dem Kurzkatalog im Journal de Genève nachweisbar, ohne Quellenverweis und Lokalisierung. Der MECOS-99 lokalisiert dieses angebliche Beben in der Schweiz und verweist auf Volger. Möglicherweise handelt es sich um eine Verwechslung mit zwei Beben in der Auvergne im März 1490 (vgl. SisFrance).

Die Lokalisierung „Lugano“ wiederum stammt aus dem Katalog von Van Gils \& Leydecker. Da dieser keine Quellenhinweise enthält, kann über die Herkunft höchstens spekuliert werden. Im italienischen Katalog (DOM4.1), in Baratta (1901) und Guidoboni (2005b) ist zu diesem Datum kein Erdbeben zu finden. Es wäre noch zu überprüfen, ob es sich um eine Verwechslung mit dem in Candreia $(1905,28)$ aufgeführten Beben vom 13. Juni $1491 \mathrm{im}$ Val S. Giacomo (Splügenpass) handelt. Er stützte sich dabei auf Chiaverini (1667), was hier nicht verifiziert werden konnte. Bei dieser unklaren Datenlage muss deshalb vorläufig auf eine Falschmeldung geschlossen werden.

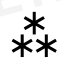




\section{Datum Zeit Ort \\ 1492, 7. November $\quad-\quad$ Aesch-Basel}

\section{Bewertung des Ereignisses}

Kein Erdbeben: Meteoritenfall in Ensisheim, Elsass.

\section{Überlieferung $u$. a. in:}

- Gross (1624)

- Bertrand (1756)

- $\quad$ Hoff (1840-1841)

- $\quad$ olger (1857)

- Sieberg (1940)

- MECOS-99

\section{Quelle:}

Knape \& Wuttke (1990): Einblattdruck von Sebastian Brandt, 1492

\section{Interpretation}

Beim Ereignis vom 7. November 1492 handelt es sich nachweislich um einen Meteoriten, der bei Ensisheim im Elsass mit einem lauten Knall aufschlug und eine weitherum wahrnehmbare Erschütterung verursachte, was später irrtümlich zur Interpretation als Erdbeben führte (Gross, 1624). Von Sebastian Brandt, Verfasser des bekannten Narrenschiffes, ist ein Einblattdruck mit Holzschnitt überliefert. Es wird auch darüber gerätselt, ob das Bild auf der Rückseite des „Büssenden Heiligen Hieronymus“ aus dem Jahre 1494 oder 1497 von Albrecht Dürer in der National Gallery in London dieses Ereignis darstelle (Böhme, 2004). Dürer hat sich zu dieser Zeit im Elsass und in Basel aufgehalten.

\section{Publikationen, u. a. in:}

- Von der Mühll (1975)

- Schwarz-Zanetti (2008d), Exkurs

\begin{tabular}{|c|c|c|}
\hline $\begin{array}{l}\text { Datum } \\
\text { 1498, 31. März }\end{array}$ & $\begin{array}{l}\text { Zeit } \\
-\end{array}$ & $\begin{array}{l}\text { Ort } \\
\text { Zweisimmen }\end{array}$ \\
\hline
\end{tabular}

\section{Bewertung des Ereignisses}

Kein Erdbeben: Datierungs- und Lokalisierungsfehler 


\section{Korrektur}

April 1498 in Saanen.

\section{Überlieferung}

Van Gils \& Leydecker (1991)

\section{Quelle:}

Binder (1905-06) basiert vermutlich auf der zeitgenössischen Chronik „Der Schwabenkrieg (1849)“ von Hans Lenz, hsg. von H. von Diessbach (1849).

\section{Interpretation}

Diese Datierung und Lokalisierung finden sich nur im Katalog von Van Gils \& Leydecker (1991), vermutlich handelt es sich um einen Interpretationsfehler der zeitgenössischen Quelle von Hans Lenz in Binder (1905-06).

\section{Bemerkungen}

Vgl. im ECOS-09 die zwei Erdbeben vom April und 3. September 1498 in Saanen, BE.

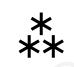
Datum
Zeit (UTC)
Ort
- 1498, April
- 9 Uhr
- Nicht lokalisierbar (Saanen, BE)
- 1498, 3. September
- 14 Uhr
- Nicht lokalisierbar (Saanen, BE)

\section{Bewertung des Ereignisses}

Unsichere Ereignisse.

\section{Parameter nach ECOS}

- April lx: 5

- 3. September Ix: 6

\section{Überlieferung}

- $\quad$ Binder (1905-1906)

- MECSO-99

\section{Quellen}

Der Quellenbeleg wurde von Binder (1905-06) publiziert, aber nur mit einem allgemeinen Hinweis auf Hans Lenz, der um 1498 Lehrer in Saanen war, und bekannt ist als Verfasser der Schwäbischen Reimchronik „Der Schwabenkrieg“. Diese Chronik wurde 1849 von Diessbach herausgegeben. 


\section{Diessbach (1849, S. 23-24):}

Von den erdbidemen, so desselben sommers und jar geschachen zu Sana und Wallis und doumb 23
[1498] „(...) In dem monat Abrellen
Dett ein erdbidem erschellen
Zwüschend der 10. und 11. stund.
Ist war und menklichen kunt,
Wüssen und offenbar,
(...)
Darnach im September in frist
Der erst Herpstmonet genant
Am dritten tag ward mir bekannt
Zwüschen 3 und 4 nachmittag
By hochen sonnenschyn ich sag
Ein erdbidem erschrockenlich,
Das die techer erschütten sich.
Zur erd harnyder viel ich“.

\section{Interpretation}

Der Beobachter Hans Lenz in Saanen spricht nicht nur von Erschrecken, sondern auch von Erschüttern und Umfallen, sodass das Beben mit einer maximalen Intensität von 6 gewertet wird, allerdings mit "unsicher“, da nur ein einziger Beleg vorhanden ist.

\section{Makroseismische Parameter}

April 1498

\begin{tabular}{|l|l|l|l|l|l|l|}
\hline Ortschaft & Länge & Breite & $I_{\min }$ & $I_{\max }$ & $I_{\text {w }}$ & Datenqualität \\
\hline Saanen & 46.49 & 7.26 & 4 & 5 & 5 & sehr schwach \\
\hline Kanton Wallis & 46.26 & 7.62 & & & verspürt & sehr schwach
\end{tabular}

3. September 1498

\begin{tabular}{|l|l|l|l|l|l|l|}
\hline Ortschaft & Länge & Breite & $I_{\min }$ & $I_{\max }$ & $I_{\text {w }}$ & Datenqualität \\
\hline Kanton Wallis & 46.26 & 7.62 & & & verspürt & sehr schwach \\
\hline Saanen & 46.49 & 7.26 & 5 & 6 & 6 & sehr schwach \\
\hline
\end{tabular}

23 hier herum. 


\begin{tabular}{l|l|l}
\hline Datum & Zeit & Ort \\
$1500,-$ & - & Neuchâtel
\end{tabular}

\section{Bewertung des Ereignisses}

Kein Erdbeben.

\section{Korrektur}

\section{Überlieferung u. a. in:}

- Bertrand (1756)

- Perrey (1845)

- $\quad$ Volger (1857)

- Kopp (1861)

Nicht vorhanden in den Kompilationen:

- $\quad$ Tribolet (1876)

- MECOS-99

\section{Nicht vorhanden in zeitgenössischer Quelle:}

Diebold Schilling $(1513,1981)$

\section{Interpretation}

Der älteste Nachweis fand sich in der Kompilation von Bertrand (1756): „En mille-cinqcent, la terre trembla en divers lieux. Plusieurs endroits de la Suisse l'éprouverent.“ [Im Jahr 1500 \{im Druck am Rand irrtümlich: 1502\} zitterte die Erde an verschiedenen Orten. Mehrere Orte der Schweiz haben es verspürt.]

Da keine zeitgenössischen Berichte gefunden wurden, sind diese Angaben vorläufig als Falschmeldung zu werten. Vielleicht besteht auch ein Zusammenhang mit dem angeblichen Beben vom 30. April 1500.

Es ist zu erwähnen, dass bei zwei weiteren vollen Jahrhundertzahlen nicht verifizierbare Erdbeben erwähnt sind. Möglicherweise steckt eine zahlenmystische Idee dahinter; vgl. auch die Jahre 1000 und 1400.

\section{Bemerkungen}

Vgl. das angebliche Erdbeben vom 30. April bzw. Mai 1500; ebenso die Falschmeldungen zu den Jahren 1000 und 1400. 


\begin{tabular}{l|l|l} 
Datum & Zeit & Ort \\
$-1500,30$. April & - & - Einsiedeln, SZ \\
-1500, Mai & - & - Schweiz
\end{tabular}

\section{Bewertung des Ereignisses}

Keine Erdbeben.

\section{Korrektur}

\section{Überlieferung u. a. in:}

- Gils, Van \& Leydecker (1991)

Lokalisierung in der Schweiz:

- Bertrand (1756)

- Perrey (1845)

- Volger (1857)

- MECOS-99

Nicht vorhanden in zeitgenössischer Quelle:

Diebold Schilling $(1513,1981)$.

\section{Interpretation}

Ohne Quellenverweis schreiben Bertrand (1756) und seine Kopisten, dass im Mai 1500 in der Schweiz ein Erdbeben verspürt worden sei, Schäden werden keine erwähnt. Zeitgenössische Berichte sind nicht bekannt, obwohl zum Beispiel die Luzerner Chronik von Diebold Schilling (1513) mehrere andere Erdbeben anfangs des 16. Jahrhunderts erwähnt. Ohne Zeugenberichte ist vorläufig von einer Falschmeldung auszugehen.

Die Datierung „30. April 1500“ und die Lokalisierung „Einsiedeln“ tauchen erst bei Van Gils \& Leydecker (1991) auf, möglicherweise handelt es sich dabei um ihre Interpretation der oben erwähnten Information, indem der letzte Tag vor dem angegebenen Zeitbereich und Einsiedeln als zentraler Ort der Schweiz gewählt wurde.

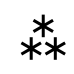

\begin{tabular}{|l|l|l}
\hline Datum & Zeit & Ort \\
\hline 1504, März & - & Ardez, GR
\end{tabular}

\section{Bewertung des Ereignisses}

Sehr unsicher.

\section{Parameter nach ECOS}

Ix: 7 


\section{Publiziert in:}

Gisler, M. et al. (2005): Erdbeben in Graubünden. Vergangenheit, Gegenwart, Zukunft. S. $60-61$.

\section{Zusammenfassung}

Der Chronist Ulrich Campell beschrieb in seiner erst 1573-76 verfassten Chronik (Plattner, 1887) einen Turm in Ardez, der im Jahre 1504 angeblich durch ein Erdbeben zerstört worden sei, wobei fünf Menschen getötet wurden. Da Campell keine Quelle dafür angibt, und zeitgenössische Quellen aus der Umgebung fehlen (gemäss telefonischer Auskunft von Jon Claglüna, Verfasser einer Geschichte von Ardez, 17.12.2010), muss dieses Ereignis zumindest vorläufig mit „sehr unsicher“ gewertet werden, denn eine andere Ursache des Zusammensturzes kann nicht ausgeschlossen werden.

\section{Makroseismische Parameter}

\begin{tabular}{|l|l|l|l|l|l|l|}
\hline Ortschaft & Länge & Breite & $I_{\min }$ & $I_{\max }$ & $I_{\text {w }}$ & Datenqualität \\
\hline Engadin & 46.65 & 10.05 & & & verspürt & sehr schwach \\
\hline Ardez & 46.77 & 10.2 & 7 & 8 & 7 & sehr schwach \\
\hline
\end{tabular}

\section{Datum}

- 1506, 7. September

- 1506, 4. Oktober
Zeit (UTC)

- um 800 Uhr

- 23 Uhr
Ort

- Nicht lokalisierbar (Basel)

- Nicht lokalisierbar (Basel)

\section{Bewertung der Ereignisse}

Unsichere Ereignisse.

\section{Parameter nach ECOS}

- 7. September Ix: verspürt

- 4. Oktober Ix: verspürt

\section{Überlieferung}

Neuer Fund.

Nicht vorhanden in:

- Bertrand (1756)

- Merian (1834)

- Volger (1857)

- MECOS-99

\section{Quellen}

- Bernoulli, A. (1902): Die Anonyme Chronik bei Schnitt, sammt Fortsetzung 14951541 (S. 192: (340): „Anno 1506 uff den 7. tag septembris, umb die 9. stund vor 
mitag, was zuo Basel ein erdbidem. Uff den 4. tag octobris, umb die 12. stund in der nacht was zuo Basel ein erdbidem.“

- Bernoulli, A. (Hrsg.), 1902: Die Chronik in Ludwig Kilchmanns Schuldbuch 14681518. „....) Und woren wol fier ertbidem ouch in dissem jor, ... .“

- Schilling, Diebold (1981): „In dem obgenanten jar [1506] und vor dissen dingen umb sant Mauritzentag (22. Sept.) kam ein sollicher grosser erdbüden gan Basel, das vil lüten besorgtend und meintend, die statt und hüser woeltend umbfallen.“

\section{Interpretation}

Nur aus der anonymen Chronik bei Schnitt sind die Tagesdaten bekannt, während mit Schillings Datierung „um den 22. September“ und der Angabe von „vier Erdbeben im Jahr 1506“ aus Kilchmanns Schuldbuch eine ungefähre Bestätigung gegeben wird. Doch weder Intensitätsumschreibungen noch Schäden werden erwähnt. Damit darf ein kleineres Erdbeben ohne Schaden in Basel im September 1506 als ziemlich gesichert gewertet werden. Da Schilling in Luzern das Beben wohl nicht miterlebt hat und die Intensität mit einer gängigen Erdbebenintensitäts-Metapher umschreibt, so muss mit „verspürt“ gewertet werden. Das Beben vom 4. Oktober ist nur aus der anonymen Chronik bei Schnitt bekannt.

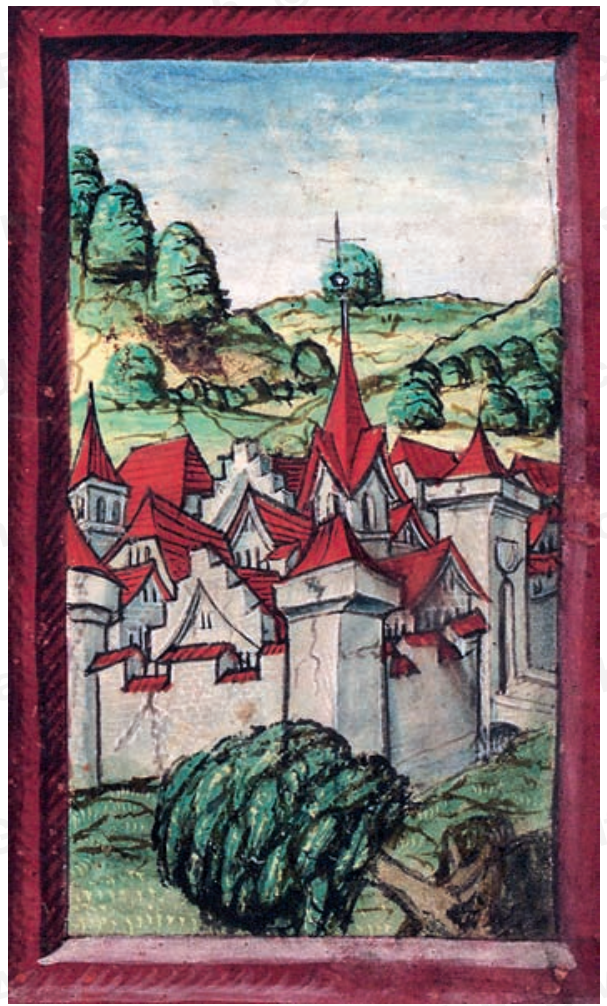

Abbildung 4: Darstellung der angeblichen Schäden des Basler Bebens vom 7. September 1506 in der Diebold Schilling-Chronik aus Luzern aus dem Jahr 1513 (1981), Copyright Korporation Luzern, 2009. 
Das farbige Bild aus der Luzerner Chronik von Diebold Schilling stammt möglicherweise vom Autoren selber. Es zeigt Risse in den Stadtmauern und einen umgefallenen Baum; Schäden, die im Text aber nicht erwähnt werden, sodass die Darstellung als fiktiv zu werten ist. Sie gehört aber zu den ältesten Erdbebenbildern der Schweiz.

\section{Makroseismische Parameter}

7. September 1506

\begin{tabular}{|l|l|l|l|l|l|l|}
\hline Ortschaft & Länge & Breite & $I_{\min }$ & $I_{\max }$ & $I_{\text {w }}$ & Datenqualität \\
\hline Basel & 47.56 & 7.59 & & & verspürt & sehr schwach \\
\hline
\end{tabular}

4. Oktober 1506

\begin{tabular}{|l|l|l|l|l|l|l|}
\hline Ortschaft & Länge & Breite & $I_{\min }$ & $I_{\max }$ & $I_{w}$ & Datenqualität \\
\hline Basel & 47.56 & 7.59 & & & verspürt & sehr schwach \\
\hline
\end{tabular}

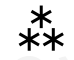

Datum

1506, 22. September

\section{Zeit}

\section{Ort}

Basel

\section{Bewertung des Ereignisses}

Kein Erdbeben: Datierungsfehler

\section{Korrektur}

1506, 7. September

\section{Überlieferung}

- Schilling, Diebold (1513, 1981)

- Bernoulli, A. (1902): Die Anonyme Chronik bei Schnitt, sammt Fortsetzung 14951541 (S. 185f.)

\section{Interpretation}

Der Luzerner Schilling gibt für Basel nur eine ungefähre Datierung an: „In dem obgenanten jar [1506] und vor dissen dingen umb sant Mauritzentag (22. Sept.) kam ein sollicher grosser erdbüden gan Basel, das vil lüten besorgtend und meintend, die statt und hüser woeltend umbfallen.“ Während die Basler Quelle bei Schnitt ausdrücklich auf den 7. September datiert, sodass der 7. September wahrscheinlicher ist.

\section{Bemerkungen}

Vgl. das Erdbeben vom 7. September 1506. 


\begin{tabular}{|l|l|l|}
\hline Datum & Zeit & Ort \\
\hline 1507, 7. September & 9 Uhr & Basel
\end{tabular}

\section{Bewertung des Ereignisses}

Kein Erdbeben: Datierungsfehler

\section{Korrektur}

1506, 7. September

\section{Überlieferung}

Nicht vorhanden in:

- Bertrand (1756)

- Volger (1857)

- MECOS-99

\section{Interpretation}

Dieser Fehler ist nicht mehr nachvollziehbar, wahrscheinlich handelt es sich um einen Abschreibfehler für das gut belegte Erdbeben vom 7. September 1506 in Basel.

\section{Bemerkungen}

Vgl. das Erdbeben vom 7. September 1506.

\begin{tabular}{|c|c|c|}
\hline $\begin{array}{l}\text { Datum } \\
1508\end{array}$ & $\begin{array}{l}\text { Zeit } \\
-\end{array}$ & $\begin{array}{l}\text { Ort } \\
\text { Albula, GR }\end{array}$ \\
\hline
\end{tabular}

\section{Bewertung des Ereignisses}

Kein Erdbeben: Lokalisierungsfehler.

\section{Korrektur}

Schweiz

\section{Überlieferung u. a. in:}

- Stumpf (1548)

- Plattner (1887), Campell

- Candreia (1905)

- Montandon (1942-43)

- Gils, Van \& Leydecker (1991)

- MECOS-99 und MECOS-02 
Nicht vorhanden in zeitgenössischer Quelle:

Schilling-Chronik von Luzern (1513/1981)

\section{Interpretation}

Es sind keine zeitgenössischen Beobachtungen nachzuweisen. Erst in Stumpfs Chronik von 1548 findet man den Hinweis auf „grosse Erdbeben in Germanien und Italien im Jahr 1508“, während der jüngere Bündner Campell (Plattner 1887) - der seine Chronik zwischen 1570 und 1582 geschrieben hatte und die Stumpf-Chronik gekannt haben dürfte -, ohne Lokalisierung „schreckliche Erdbeben“ festhält. Auf seiner Notiz basiert vermutlich die Annahme, dass im Kanton Graubünden diese Erdbeben stattgefunden hätten, wie zum Beispiel Montandon folgert (1942-43, S. 30). In Van Gils \& Leydecker taucht dann die Lokalisierung „Albula“ ohne Quellenhinweis auf. Deshalb ist diese Lokalisierung als falsch zu werten. Auch die allgemeinere Lokalisierung „Schweiz“ ist unsicher.

\section{Bemerkungen}

Vgl. in ECOS-09 das nicht näher bestimmbare Erdbeben von 1508, lokalisiert in der Schweiz.

\section{**}

\begin{tabular}{l|l|l} 
Datum & Zeit & Ort \\
-1512 & - & - Biasca \\
$-1513,10$. Februar & & - Bellinzona \\
$-1513,30$. September & & - San Bernardino
\end{tabular}

\section{Bewertung der Ereignisse}

Keine Erdbeben, sondern ein Bergsturz mit grossen Folgeschäden.

\section{Überlieferung $u$. a. in:}

- Volger (1857)

- Candreia (1905)

- Montandon (1942-43)

- MECOS-99

\section{Publiziert in:}

- Stucchi \& Albini (1988)

- Historisches Lexikon der Schweiz, http://hls-dhs-dss.ch, Artikel „Biasca“ und „Buzza di Biasca“. 
Zusammenfassung, nach dem Artikel im Historischen Lexikon der Schweiz (http://hls-dhs-dss.ch, 15. Dezember 2008):

Bergsturz vom Pizzo Magn am 30. September 1513 (es werden auch andere Tagesdatierungen genannt), der den Brenno staute, bis dann am 20. Mai 1515 das Wasser durchbrach, die tieferliegenden Tessiner Gebiete überschwemmte und zahlreiche Opfer forderte. 1517 kam es deswegen zu einem Prozess gegen die Bewohner von Malvaglia, die angeblich den Wasserausbruch durch Zauberei verursacht hätten; sie wurden aber freigesprochen.

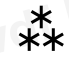

\begin{tabular}{l|l|l} 
Datum & Zeit & Ort \\
$-\quad 1517,4$. April & - & - Böblingen, D \\
$-\quad 1517$, 26. Juni & & - Nördlingen, D
\end{tabular}

\section{Bewertung des Ereignisses}

Keine Erdbeben: Sturm.

\section{Überlieferung}

- Münster (1544), ältester Nachweis

- Kopp (1861)

- Giessberger (1922)

- Sieberg (1940)

- Gils, Van \& Leydecker (1991)

- MECOS-99

Zeitgenössische Wettertagebücher ohne Erdbebenhinweise am 4. April und 26. Juni:

- Kilian Leib, Rebdorf bei Ingolstadt (Manuskript in der Staatsbibliothek München (BSB): $4 \mathrm{~L}$ Impr. C. n. mss 73, Arbeits-Transkription in Datenbank Euroclimhist SchwarzZanetti, 1992, Nr. 8858)

- Hieronymus Rose; Ingolstadt (Manuskript in der Staatsbibliothek München (BSB): Eph. Astr. 127; Arbeits-Transkription in Datenbank Euroclimhist, 1992, Nr. 8885)

- Johannes Stöffler, Tübingen (Manuskript in der Württembergischen Landesbibliothek Stuttgart (WLB): R 16 Stoe 1, Arbeits-Transkription in Datenbank Euroclimhist, 1992, Nr. 8857)

\section{Interpretation}

Die aufgeführten Kompilationen scheinen sich alle auf Sebastian Münster (1544) abzustützen, der einen Sturmwind und ein Beben am 26. Juni 1517 aufführt. Er lebte 1517 in Tübingen. Bereits Sieberg (1940) vermutet für Nördlingen eine Verwechslung mit dem Sturm. Er lokalisiert aber für diesen Zeitpunkt ein Beben in Franken, im ganzen bayrischen Fichtelgebirge und in Bayreuth, was hier nicht weiter überprüft werden konnte. 
In den zeitgleichen und akribischen süddeutschen Wettertagebüchern in der Klimadatenbank Euroclimhist-Mittelalter 1 (Schwarz-Zanetti, 1992), z. B. von Kilian Leib aus Rebdorf bei Ingolstadt, Hieronymus Rose in Ingolstadt oder Johannes Stöffler in Tübingen, sind für den 26. Juni 1517 ebenfalls keine Erdbeben, sondern nur ein Sturm verzeichnet. Dieser scheint in Nördlingen grosse Schäden verursacht zu haben. Dasselbe steht auch in der Chronik von Wilhelm Rem (1896), der von 1462-1529 lebte.

Bei Giessberger (1922) wird auch ein Erdbeben am 4. April 1517 erwähnt, für das in den süddeutschen Wettertagebüchern ebenfalls kein Beleg gefunden werden konnte. Generell ist anzumerken, dass eine Verwechslung von Stürmen und deren Schäden mit Erdbeben kein seltenes Phänomen ist, vgl. auch 1523. Die Lokalisierung in Neuenburg, $\mathrm{CH}$, ist vermutlich eine Fehlinterpretation der unkommentierten Kompilationen von Kopp (1861) oder Tribolet (1876) im MECOS-99.

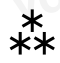
Datum
1523, 19. Mai
Zeit
Ort
Suisse romande

\section{Bewertung des Ereignisses}

Kein Erdbeben: Sturm.

\section{Korrektur}

Sturm.

\section{Überlieferung u. a. in:}

- Bertrand (1756)

- Guéneau de Montbelliard (1761)

- Hoff (1840-1841)

- Volger (1857)

- Montandon (1942-43)

- MECOS-99

Zeitgenössische Wettertagebücher mit Sturm, aber ohne Erdbebenhinweis:

- Kilian Leib, Rebdorf bei Ingolstadt (Manuskript in der Bayerischen Staatsbibliothek München (BSB): 4 L Impr. C. n. mss 73, Arbeits-Transkription in Datenbank Euroclimhist Schwarz-Zanetti, 1992, Nr. 8858)

- Hieronymus Rose; Ingolstadt (Manuskript in der Bayerischen Staatsbibliothek München (BSB): Eph. Astr. 127; Transkription in Datenbank Euroclimhist, 1992, Nr. 8885)

- Johannes Stöffler, Tübingen (Manuskript in der Württembergischen Landesbibliothek Stuttgart (WLB): R 16 Stoe 1, Arbeits-Transkription in Datenbank Euroclimhist, 1992, Nr. 8857) 


\section{Interpretation}

Im MECOS-99 wird dieses angebliche Beben auf eine Intensität 6 geschätzt, in Montandon sogar auf 7. Der Hinweis lässt sich aber nur bis auf Bertrand (1756) zurückverfolgen, wo dieses Beben mit zwei Druckfehlern (1323 und 1503) aufgeführt ist. Es werden jedoch keine Schäden erwähnt. In den zeitgenössischen süddeutschen Wettertagebüchern (Schwarz-Zanetti, 1992 und Euroclimhist) findet sich hingegen genau an diesem Datum erneut ein Sturm, vgl. 26. Juni 1517, sodass mit grösster Wahrscheinlichkeit auch hier von einer Verwechslung des Bebens mit einem Sturm ausgegangen werden kann.

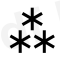

\begin{tabular}{|l|l|l}
\hline Datum & Zeit (UTC) & Ort \\
\hline 1523, 27. Dezember & 23 Uhr & Freiburg i. Br., D
\end{tabular}

\section{Bewertung des Ereignisses}

Sicheres Ereignis.

\section{Parameter nach ECOS}

Ix: 6.5

\section{Überlieferung}

- $\quad$ Lycosthenes (1557): Beben am 27. Dezember 1533 (sic)

- Volger (1857)

- Langenbeck (1892)

- Naegele (1911)

- Sieberg (1940)

- $\quad$ Vogt (1992)

- MECOS-99

- SisFrance (September 2010)

\section{Quellen}

- Die Anonyme Chronik der Mailänderkriege 1507-1516 (Bernoulli, 1902, S. 85): „Von einem erdbidem. Uff sanct Johans tag zu wyhenachten anno 1523 kamen dry grosz erdbidem nach einander. [Anm. Herausgeber: In der Nacht vor dem 27. Dec. 1523, gegen 1 oder 2 Uhr; vgl. B. Chron. I, 47 und 387.]“

- Die Chronik des Fridolin Ryff 1514-1541 (Vischer, 1872): „Von eim grosen erdbydem. Uff der kindlen tag zu nacht zwischen zwelffen und ein im 152x (unklare Zuordnung in der Edition, es können die Jahre 1523 oder 1524 gemeint sein) jor kam ein groser erdbydem, das die huser erziderten. Man seit ouch, das er by sechsz mil wegs wyd gehört wer worden, vergieng schnel wyder. 
- Vogt (1992) zitiert aus einer Arbeit von Nägele (1911), die hier nicht eingesehen werden konnte, einen Brief aus Freiburg i.B. an den Ulmer Arzt Rychard: „C'est bien la nuit de la Saint-Jean [1523], vers minuit, que le séisme survient avec une violence telle que les Franciscains tombent à terre lors de l'office. (...) Nous apprenons que toute la ville oscille. (...) Bien plus, est signalée la chute de nombreuses pierres."

- Vogt (1992) fand keine Elsässer Quellen zu diesem Ereignis.

Zeitgenössische süddeutsche Wettertagebücher ohne Erdbebenhinweis

(Schwarz-Zanetti, 1992):

- Kilian Leib, Rebdorf bei Ingolstadt (Manuskript in der Bayerischen Staatsbibliothek München (BSB): 4 L Impr. C. n. mss 73, Arbeits-Transkription in Datenbank Euroclimhist Schwarz-Zanetti, 1992, Nr. 8858)

- Johannes Stöffler, Tübingen (Manuskript in der Württembergischen Landesbibliothek Stuttgart (WLB): R 16 Stoe 1, Arbeits-Transkription in Datenbank Euroclimhist, 1992, Nr. 8857)

\section{Interpretation}

Die Hauptinformationen stammen aus Nägele (1911), der den Freund des Ulmer Arztes Rychard aus Freiburg i.B. zitiert, wo kleinere Schäden entstanden seien, und aus zwei Basler Quellen, die keine Schäden erwähnen. Im Elsass sind bisher keine Beobachtungen nachweisbar, ebenso wenig in den süddeutschen Wettertagebüchern, so z. B. in Tübingen und Rebdorf bei Eichstätt, Bayern (Schwarz-Zanetti, 1992).

\section{Makroseismische Parameter}

\begin{tabular}{|l|l|l|l|l|l|l|}
\hline Ortschaft & Länge & Breite & $I_{\min }$ & $I_{\max }$ & $I_{\text {w }}$ & Datenqualität \\
\hline Freiburg i. B., D & 48 & 7.87 & 6 & 7 & 6 & sehr schwach \\
\hline Basel & 47.56 & 7.59 & 4 & 5 & 5 & sehr schwach \\
\hline Tuebingen, D & 48.53 & 9.06 & 1 & 3 & 1 & sehr schwach \\
\hline Rebdorf, D & 48.87 & 11.17 & 1 & 3 & 1 & sehr schwach \\
\hline
\end{tabular}

\section{**}

Datum

1524, April
Zeit

$-$
Ort

Ardon, VS

\section{Bewertung des Ereignisses}

Sicher.

\section{Parameter nach ECOS}

Ix: 8 


\section{Makroseismische Parameter}

\begin{tabular}{|l|l|l|l|l|l|l|}
\hline Ortschaft & Länge & Breite & $I_{\min }$ & $I_{\max }$ & $I_{w}$ & Datenqualität \\
\hline Ardon & 46.21 & 7.26 & 7 & 8 & 8 & schwach \\
\hline Savièse & 46.25 & 7.35 & 7 & 8 & 7 & sehr schwach \\
\hline Conthey & 46.23 & 7.31 & 7 & 8 & 7 & sehr schwach \\
\hline Basel & 47.56 & 7.59 & 4 & 5 & 5 & sehr schwach \\
\hline
\end{tabular}

\section{Publiziert in:}

- Kurzfassung in: Schwarz-Zanetti (2008g)

- Schwarz-Zanetti, G. et al. (2011, in prep.)

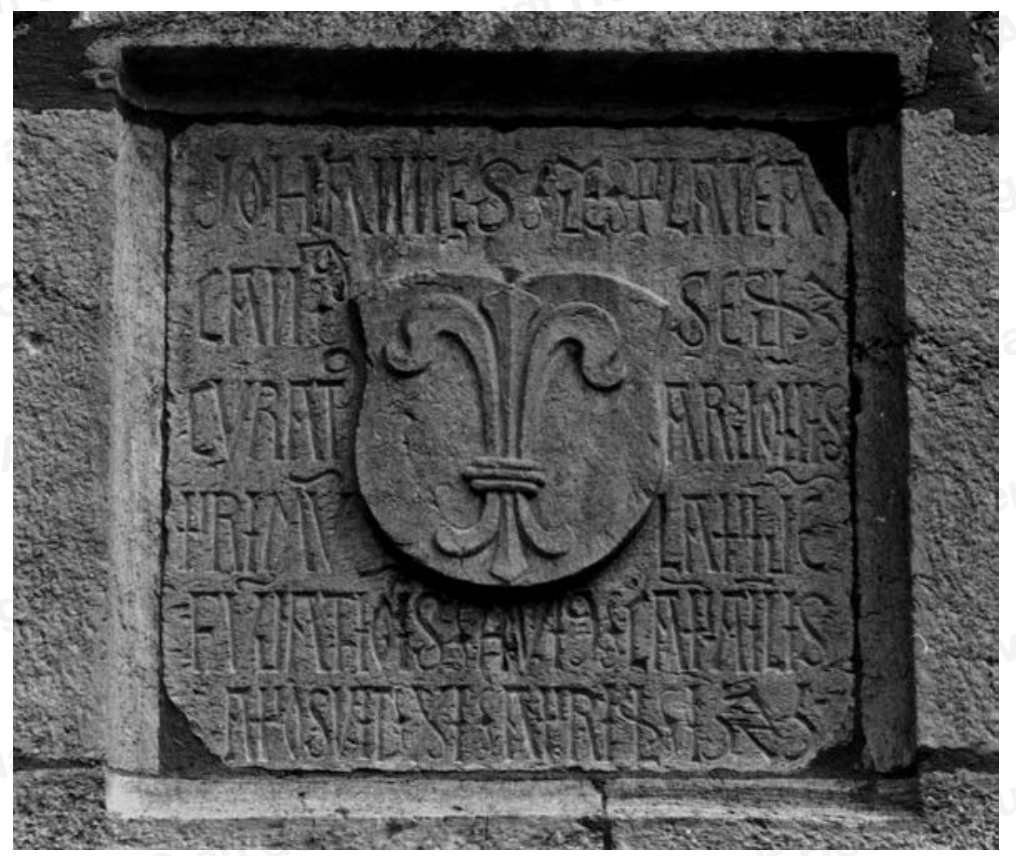

Abbildung 5: Steintafel am heutigen Kirchturm von Ardon anlässlich seiner Grundsteinlegung im Jahr 1525

„Johannes de Platea, canonicus Sedunensis, curatus Ardonensis, primum lapidem fundationis hujus campanilis aposuit XI aprilis 1525.“ [Johannes von Platea, Domherr von Sion, Pfarrer von Ardon, hat den ersten Grundstein dieses Turms am 11. April 1525 gelegt.] Der eigentliche Grund des Neubaus wird nicht erwähnt, doch die zeitgenössische Quelle aus Monthey mit der Erwähnung von Schäden am Kirchturm Ardons legt einen Zusammenhang mit dem Erdbeben vom April 1524 nahe. 


\section{Zusammenfassung}

Bei diesem Schadenbeben handelt es sich um einen neuen Fund. Eine zeitgenössische Notiz des Notars Revilliodi von Monthey berichtet von einem Erdbeben im April 1524, das die Kirchtürme in Ardon, Savièse und Plan-Conthey beschädigte (Santschi, 1966), was von einer Inschrift aus dem Jahr 1525 anlässlich des Turmneubaus von Ardon teilweise bestätigt wird (Gaillard, 1996). Zusätzlich weisen mehrere archäologische Befunde aus Untersuchungen von Walliser Kirchen auf Renovationen im 2. Viertel des 16. Jahrhunderts hin. Möglicherweise besteht auch ein Zusammenhang mit einer Basler Erdbeben-Beobachtung vom 20. April 1524, vgl. MECOS-99.

\section{Bemerkungen}

In älteren Kompilationen, so zum Beispiel im MECOS-99, ist ein Erdbeben am 20. April 1524 in Basel verzeichnet, wahrscheinlich basierend auf Ryff (1872, S. 48).

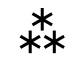

\begin{tabular}{|l|l|l}
\hline Datum & Zeit & Ort \\
\hline 1524, 27. Dezember & - & Basel
\end{tabular}

\section{Bewertung des Ereignisses}

Kein Erdbeben: Datierungsfehler.

\section{Korrektur}

1523, 27. Dezember

\section{Überlieferung u. a. in:}

- Volger (1857)

- Sieberg (1940)

- MECOS-99

\section{Interpretation}

Der Fehler entstand durch eine unklare Zuordnung der Beobachtung zur Datierung in der Edition der Chronik des Fridolin Ryff 1514-1541 (Vischer, 1872): 1523 oder 1524. Das Epizentrum des Bebens vom Dezember 1523 muss in der Region von Freiburg im Breisgau, D, gelegen sein.

\section{Bemerkungen}

Vgl. im ECOS-09 das Erdbeben vom 27. Dezember 1523.

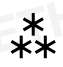




\begin{tabular}{|l|l|l}
\hline Datum & Zeit & Ort \\
1531, 26. Januar & - & Schweiz
\end{tabular}

\section{Bewertung des Ereignisses}

Kein Erdbeben: Datierungsfehler.

\section{Korrektur}

1531, 10. Oktober, St. Gallen (oder 26. Januar 1531 in Lissabon)

\section{Überlieferung}

Eine mögliche Verwechslung des Januar-Bebens von Lissabon mit dem Oktober-Beben in St. Gallen scheint den meisten Kompilatoren zwar bewusst gewesen zu sein, aber nicht immer wurde daraus der entsprechende Schluss gezogen.

- Rasch (1591)

- Bertrand (1756)

- Volger (1857)

- Sieberg (1940)

- Montandon (1942-43)

- Rothé \& Schneider (1968)

- MECOS-99

\section{Interpretation}

Das Lissabonner Beben vom 26. Januar 1531 war in der Erdbebenliteratur bald einmal bekannt, so bereits bei Rasch (1591). Wegen des nur neun Monate später erfolgten Bebens in der Ostschweiz, am 10. Oktober 1531, kamen trotzdem Verwechslungen vor (vgl. die Originalquellen zu 10.10.1531). Ähnliche Verwechslungen geschahen auch rund um das grosse Erdbeben von Lissabon vom 1. November 1755 und dem Beben vom 9. Dezember 1755 in Brig-Naters.

\section{Bemerkungen}

Vgl. das Erdbeben vom 10. Oktober 1531 in St. Gallen.

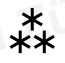

\section{Datum}

1531, 10. Oktober

\section{Zeit (UTC)}

$20 \mathrm{Uhr}$
Ort

St. Gallen

\section{Bewertung des Ereignisses}

Gesichertes Ereignis. 


\section{Parameter nach ECOS}

Ix: 5

\section{Überlieferung}

- Rasch, (1591), Deutschland

- Amberg (1892)

- Candreia (1905)

- Montandon (1942-43)

- Rothé \& Schneider (1968)

\section{Quellen}

- Tschudi Aegidius, Kappelerkrieg, (Liebenau 1905), Sargans: „Und desselben 10. tags Oktobris, um die nüne nochmittag in der nacht, kam ein starker erdbidem der das Land, ouch berg und thal, gewaltig erschütt, und im selben augenblick hort uf der comet am himel zeerschine, der mer dann ain halb jar, mit ainer langen ruten, wie ein besen, geschinen hat.“

- Salat (1986), Luzern

- Kessler (1866), St. Gallen

- Vadian (1879), St. Gallen

- Paracelsus (1531), St. Gallen

- Rütiner (1996), St. Gallen

\section{Interpretation}

Sechs zeitgenössische Quellen, davon vier aus St. Gallen, eine aus Sargans und eine aus Luzern, erlauben eine zuverlässige Datierung des Bebens auf den 10. Oktober 1531, ungefähr um 9 Uhr abends (bzw. 20 Uhr UTC). Die Häuser erzitterten, sodass die Leute erschraken. Es wird jedoch nirgends ein Schaden erwähnt, obwohl sich vor der kurzen theologischen Abhandlung von Paracelsus (1531) über Erdbeben anlässlich des Oktober-Bebens ein Holzschnitt mit einem vermutlich fiktiven zerfallenden Turm befindet. Dieses Bild gehört zu den ältesten zeitgenössischen Erdbeben-Darstellungen der Schweiz. Eine zusätzliche Sensibilisierung der Zeitgenossen durch das Lissaboner Beben vom 26. Januar 1531 und durch eine Explosion eines Pulverhauses in St. Gallen „Espa“ (vielleicht handelt es sich hier um das Espenmoos) am frühen Morgen des 10. Oktobers (Kessler, 1911) ist wahrscheinlich.

Mentalitätsgeschichtlich interessant könnte auch eine weitere Suche nach Interpretationen der zeitlichen Abfolge des Erdbebens und des Zweiten Kappelerkrieges sein. Denn am 9. Oktober 1531 wurde den Zürchern von den Innerschweizer Orten der Krieg erklärt, am 10. Oktober bebte die Erde und am 11. Oktober 1531 wurde die Zweite Kappeler Schlacht ausgefochten, worin der Reformator Zwingli fiel und die Zürcher verloren. 
Makroseismische Parameter

\begin{tabular}{|l|l|l|l|l|l|l|}
\hline Ortschaft & Länge & Breite & $I_{\min }$ & $I_{\max }$ & $I_{\text {w }}$ & Datenqualität \\
\hline Kappel am Albis & 47.23 & 8.53 & 5 & & - & sehr schwach \\
\hline Luzern & 47.05 & 8.29 & & & verspürt & sehr schwach \\
\hline St. Gallen & 47.43 & 9.4 & 4 & 5 & 5 & gut \\
\hline Sargans & 47.05 & 9.44 & 4 & 5 & 5 & sehr schwach \\
\hline
\end{tabular}

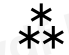

\section{Datum}

1533, 22. Oktober

\section{Zeit}

$-$

\section{Ort}

Zürich

\section{Bewertung des Ereignisses}

Kein Erdbeben: Datierungsfehler.

\section{Korrektur}

Eventuell 12. Oktober 1534, Zürich

\section{Überlieferung in:}

- Scheuchzer (1716)

- MECOS-99

1533-Datum nicht vorhanden in:

- Guggenbühl (1856/57), hsg. von Wolf.

- ZBZ: Ms B 258: Guggenbühl-Chronik

Korrekturvorschläge gemäss:

- Guggenbühl-Chronik (ZBZ: Ms B 258), entstanden 1688-92:

- „1534. (...) Den 22. winmonat [Weinmonat: Oktober] erschütete ein erdbidem die gebeuw so starck, dass vill wächter ab den thürmen geloffen. Hat doch nichts geschadet."

- Stumpf 1548, S. 164b: 12. Oktober 1534.

\section{Interpretation}

Die Falschmeldung 1533 ist erst im MECOS-99 entstanden. Denn in der Edition von Guggenbühl durch Wolf ist das Datum nicht zu finden, ebenso wenig in der Originalchronik. Dafür gibt es in letzterer genau ein Jahr später einen entsprechenden Eintrag.

Zum Oktober 1534 existieren aber weitere Daten, deren Tagesdatierung sich noch nicht ganz klären liess. Stumpf (1548, S. 164b): „Erdbidem 1534. Ein Erdbidem erzeiget sich 
vmb die statt Zürych vnd darinn/am 12. tag Octobris in der nacht.“ Ähnlich lautet die Notiz bei Scheuchzer (1746), er irrt sich aber im Datum und fügt einen Sturm am 21. und 22. Oktober hinzu: „A. 1534 den 2. Oct. in der nacht erzeigte sich ein Erdbeben um die Stadt Zürich, und darinn; darauf folgete den 21. und 22. Oct. ein starcker Sturmwind, der that in wäldern und sonst grossen Schaden. Id. Lib. VI. c. 20.“

Es kann hier nur spekuliert werden, ob Guggenbühl im 17. Jahrhundert einen Lesefehler machte oder ob es sich um eine Verwechslung mit dem erwähnten Sturm handelt.

\section{Bemerkungen}

Vgl. das Erdbeben vom 12. Oktober 1534 in Zürich.

\section{**}

\begin{tabular}{l|l|l} 
Datum & Zeit & Ort \\
1533, 17. November & gegen Mitternacht & St. Gallen
\end{tabular}

\section{Bewertung des Ereignisses}

Sicheres Ereignis.

\section{Parameter nach ECOS}

Ix: 5

\section{Überlieferung u. a. in:}

- Candreia (1905): zitiert Ghönneirus (1585), Pseudonym des Baslers Niklaus Höniger (1548-1598).

- Beuther (1601), stützt sich dabei auf einen nicht mehr eruierbaren Fortsetzer von Sleidanus (1506-1556).

- Haltmeyer (1683)

- Walser (1740)

- $\quad$ Naef (1850)

- Volger (1857)

- Brügger (1876)

- Candreia (1905)

- Giessberger (1922)

- Pfister (1984): Climhist

\section{Quellen}

- Johannes Kessler (1911), Chronik „Sabbata“, 1525-1539

- Stumpf (1547/48), aus dem Kapitel vom St. Galler Vadian

- Campell (ca. 1570 verfasst, hsg. von Plattner [1887]) 


\section{Interpretation}

Die St. Galler Kessler und Vadian erwähnen ein Erdbeben und einen Erdrutsch am Fluss Sitter in der Nähe von Bernhardzell im November 1533. Dabei datiert der näher am Ereignis schreibende Kessler auf den 17. November, die späteren Stumpf, bzw. Vadian, und Campell aber auf „um den 26. November“, weshalb, zumindest vorläufig, auf den 17. November datiert wird. Auch der Fortsetzer der Chronik von Johannes Sleidanus (1570) datiert auf den 17.11.

Die Kopisten, die sich auf weitere unbekannte Quellen stützen, schreiben zusätzlich von einem gleichzeitigen Sturmwind im ganzen Rheintal. Für denselben Zeitpunkt sind in einer unsicheren Chronik aus dem elsässischen Thann Schneeschäden verzeichnet, der Winter 1533/34 gilt insgesamt als hart (Pfister, 1984). Da weitere Erdbebenschäden nicht bekannt sind, müssen bei der Ursache des Erdrutschs auch Niederschläge zumindest als zusätzliche Auslöser in Betracht gezogen werden. Es ist Vadian in der Stumpf-Chronik (1548), der als erster einen Zusammenhang zwischen dem Beben und der Rutschung herstellt. Während Kessler, der seine Informationen laufend festhielt, keine Verbindung zwischen dem Erdbeben und dem Erdrutsch macht, sodass der Erdrutsch in Bernhardzell als direkte Folge des Erdbebens als nicht gesichert angesehen werden muss.

\section{Makroseismische Parameter}

\begin{tabular}{|l|l|l|l|l|l|l|}
\hline Ortschaft & Länge & Breite & $I_{\min }$ & $I_{\max }$ & $I_{\text {w }}$ & Datenqualität \\
\hline Bischofszell & 47.5 & 9.19 & & & verspürt & sehr schwach \\
\hline St. Gallen & 47.43 & 9.4 & 4 & 5 & 5 & schwach \\
\hline Bernhardzell & 47.47 & 9.34 & 5 & 6 & 5 & sehr schwach \\
\hline Chur & 46.86 & 9.53 & 3 & 5 & 4 & sehr schwach \\
\hline Bludenz, A & 47.15 & 9.82 & 3 & 5 & 4 & sehr schwach \\
\hline Feldkirch, A & 47.24 & 9.6 & 3 & 5 & 4 & sehr schwach \\
\hline Brand bei Bludenz, A & 47.1 & 9.74 & 3 & 5 & 4 & sehr schwach \\
\hline
\end{tabular}

\begin{tabular}{l|l|l}
\hline Datum & Zeit & Ort \\
$-1533,17$. November & - & $-\quad$ Chur \\
$-\quad 1533$, 26. November & $-\quad$ O Uhr 30 & - St. Margrethen, SG
\end{tabular}

\section{Bewertung des Ereignisses}

Kein Erdbeben: Datierungs- und Lokalisierungsfehler.

\section{Korrektur}

1533, 17. November, St. Gallen 


\section{Überlieferung}

- Candreia (1905)

- Gils, Van \& Leydecker (1991)

- MECOS-99

- INGV-Liste (2000)

\section{Interpretation}

In der internen Erdbebenliste des INGV vom Oktober 2000 wird dieses Ereignis mit der Lokalisierung „St. Margrethen“ aufgeführt, angeblich auf dem MECOS-99 basierend, wo jedoch nur "Rheintal“ zu finden ist, basierend auf Candreia (1905). In Candreia (S. 30) findet sich auch ein Hinweis aus Campell (ca. 1570), der für "Curiam Rhaetorum“ am 26. November ein Erdbeben mit sehr starkem Wind verzeichnet. Dieser wiederum zitiert eine unbekannte Fortsetzung von Sleidanus, die den 16. November nennt. Van Gils \& Leydecker lokalisieren dieses Ereignis in der benachbarten Ortschaft Diepoldsau, SG. Eine zeitgenössische Quelle konnte nicht eruiert werden, sodass es sich mit grösserer Wahrscheinlichkeit um einen Irrtum im Zusammenhang mit dem gut belegten Erdbeben vom 17. November 1533 in St. Gallen handeln muss.

\section{Bemerkungen}

Vgl. das Erdbeben vom 17. November 1533, St. Gallen.

\begin{tabular}{|c|c|c|}
\hline $\begin{array}{l}\text { Datum } \\
\text { 1534, 2. Oktober }\end{array}$ & Zeit & $\begin{array}{l}\text { Ort } \\
\text { Baden }\end{array}$ \\
\hline
\end{tabular}

\section{Bewertung des Ereignisses}

Kein Erdbeben: Datierungsfehler.

\section{Korrektur}

1534, 12. Oktober

\section{Überlieferung}

- Stumpf (1548)

- Ragor (1578)

- Spleiss (17. Jh., StASH, ohne Sign.)

- Scheuchzer (1716/1746)

- Volger (1857)

- Montandon (1942-43)

\section{Interpretation}

Drei Erdbeben sind für Anfang Oktober 1534 überliefert, aber noch nicht ganz gesichert. So zwei Beben am 6. und eines am 12. Oktober (12./13.). Das Datum 2. Oktober 
stammt aus Scheuchzer, der sonst wörtlich bei Stumpf abgeschrieben hat, sodass es sich beim 2. Oktober um einen Druckfehler für den 12. Oktober handeln muss. In der kompilatorischen Chronik von Schaffhausen von Hans Jakob Spleiss wird auf den 13. Oktober datiert; eventuell handelt es sich um die Nacht zwischen dem 12. und 13. Oktober. Die Lokalisierung „Baden“ stammt aus den Kompilationen von Hoff (1842) und Ragor (1578) und kann hier nicht bewertet werden.

\section{Bemerkungen}

Vgl. die Erdbeben vom 22. Oktober 1533 und vom 12. Oktober 1534.

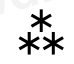

\begin{tabular}{|l|l|l}
\hline Datum & Zeit & Ort \\
\hline $1537,1$. März & - & Basel
\end{tabular}

\section{Bewertung des Ereignisses}

Kein Erdbeben.

\section{Überlieferung}

- Lycosthenes (1557)

- Volger (1857)

- Langenbeck (1892)

- Sieberg (1940)

- Gils, Van \& Leydecker (1991)

- MECOS-99

Nicht in zeitgenössischen Quellen:

- $\quad$ Ryff (Vischer, 1872)

- $\quad$ Stumpf (1548)

\section{Interpretation}

Die Jahresangabe ist seit längerer Zeit belegbar, das Tagesdatum taucht aber erstmals bei Sieberg (1940) auf: „1537, März 1, Erdbeben in Basel, VI?, bis nach Pfirt²4 verspürt“. Da Sieberg keinen Quellenhinweis gibt und weder in zeitgenössischen Dokumenten noch in Kompilationen dieses Datum gefunden werden konnte, handelt es sich mit grosser Wahrscheinlichkeit um einen Irrtum. Kein Korrekturvorschlag möglich.

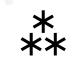

24 Pfirt: nahe Basel? 


\begin{tabular}{|l|l|l|}
\hline Datum & Zeit & Ort \\
\hline 1538, 28. Januar & - & Basel \\
\hline
\end{tabular}

\section{Bewertung des Ereignisses}

Kein Erdbeben: Datierungsfehler.

\section{Korrektur}

1538, 20. Januar

Überlieferung mit dem Datum 28. Januar u. a. in:

- Bertrand (1756)

- Volger (1857)

Überlieferung mit dem Datum 20. Januar u. a. in:

- Lycosthenes (1557)

- Burgauer (1651)

\section{Zeitgenössische Quellen}

- Bernoulli (1902): Anonyme Chronik bei Schnitt, 1495-1541

- Ryff, 1514-41 (Vischer, 1872)

\section{Interpretation}

Die zwei zeitgenössischen Quellen datieren das Erdbeben auf den 20. Januar, ebenso wie der zeitlich nahe Lycosthenes. Bertrand ist der älteste Kompilator, der auf den 28. Januar umdatiert. Da sich bei inm häufig Druckfehler belegen lassen, so ist auch hier ein Irrtum des Druckers wahrscheinlich.

\section{Bemerkungen}

Vgl. im ECOS-09 das Erdbeben vom 20. Januar 1538.

$$
\text { ** }
$$

Datum

1541, 6. Januar

\section{Zeit}

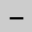

Ort

Nicht lokalisierbar (Urseren, UR)

\section{Bewertung des Ereignisses}

Unsicheres Erdbeben.

\section{Parameter nach ECOS}

Ix: verspürt 


\section{Überlieferung}

- Amberg (1892)

- Schaller-Donauer (1937?)

- Montandon (1942-43)

- MECOS-99

- Nicht in Candreia (1905).

\section{Quelle}

- Baechtold, J. (1876): Salat

\section{Interpretation}

Montandon bezieht sich irrtümlich auf Candreia, wo aber keine Notiz zu 1541 zu finden ist; vermutlich meint er Amberg (1892). Dieser stützt sich auf das Tagebuch von Hans Salat (Bächtold 1876): 1540. „(...) Item uf sant Jost's tag (St. Jodokus) in der Nacht nach 12 ist ein erdbidem gsin. = 13. Dec. (also 14.12.) “ 1541: „Item um $3 \mathrm{~m}$ regum (3-König [6. Januar]) fiengs an schnyen und regnen, kam der schützen brunnen wider - was ouch ein erdbidem gsin. Item im Tal zuo Urseren hats um 3 regum angfangen erdbidmen und me denn ein manat all tag gangen, was inen ouch ir brunn abgangen, der kam vom bidmen wider, truog etlich tag sand."

Das Jahr 1540 gilt als eines der heissesten und trockensten Jahre des letzten Jahrtausends (vgl. Pfister Climhist, 1984), somit muss das Neueinsetzen der Quellen kein Hinweis auf die Intensität des Bebens sein. Es handelt sich um einen Erdbebenschwarm mit täglichen Beben, ungefähr vom 6. Januar bis mindestens 6. Februar.

Makroseismische Parameter

\begin{tabular}{|l|l|l|l|l|l|l|}
\hline Ortschaft & Länge & Breite & $I_{\min }$ & $I_{\max }$ & $I_{\text {w }}$ & Datenqualität \\
\hline Andermatt & 46.64 & 8.6 & & & verspürt & sehr schwach \\
\hline
\end{tabular}

\section{$\stackrel{*}{* *}$}

Datum

1542, 8. November

Zeit (UTC)

$8 \mathrm{Uhr}$
Ort

Leutkirch, D

\section{Bewertung des Ereignisses}

Gesichertes Ereignis.

\section{Parameter nach ECOS}

Ix: - 


\section{Überlieferung}

- Giessberger (1922)

- Sieberg (1940)

- MECOS-99

\section{Quellen}

- Hölz (1993, S. 463 f.): „Den 8 hora 9 ante meridiem ist alhie ein grosser terramotus gesein“ (f. 38r).

- StASH, ohne Sign.: Chronik der Stadt Schaffhausen, von Hans Jakob Spleiss, Manuskript, entstanden um 1570: „Den 8. Novembris, morgens umb 8 uhren war ein grosser Erdbidem. “

\section{Interpretation}

In den zeitgenössischen Ephemeriden des Abtes Johann Christoph Raittner von Weingarten (Hölz, 1993) wird am 8. November 1542 ein Erdbeben verzeichnet. Ein Datum, das von der Spleiss-Chronik aus Schaffhausen um 1570 bestätigt wird. Giessberger (1922), der die Memminger Chronik von Schorer aus UIm (1660) zitiert, nennt dasselbe Datum und stützt sich dabei auf Chroniken der Stadt Memmingen, D, sodass mit einer grossen Wahrscheinlichkeit auf ein Erdbeben am 8. November 1542 im Gebiet von Weingarten, Memmingen, Leutkirch (Baden-Württemberg) und Schaffhausen geschlossen werden darf. Es werden aber nirgends Schäden erwähnt, die einzige zeitgenössische Intensitätsbeschreibung, aus Weingarten, spricht von "gross“.

\section{Makroseismische Parameter}

\begin{tabular}{|l|l|l|l|l|l|l|}
\hline Ortschaft & Länge & Breite & $I_{\min }$ & $I_{\max }$ & $I_{\text {w }}$ & Datenqualität \\
\hline Kempten & 47.72 & 10.31 & 4 & 6 & 5 & sehr schwach \\
\hline Schaffhausen & 47.7 & 8.64 & 5 & 6 & 5 & sehr schwach \\
\hline Weingarten & 47.81 & 9.64 & 5 & 6 & 5 & sehr schwach \\
\hline Memmingen & 47.98 & 10.18 & 5 & 6 & 5 & sehr schwach \\
\hline Lindau am Bodensee & 47.56 & 9.7 & 4 & 6 & 5 & sehr schwach \\
\hline Allgäu & 47.5 & 10.25 & 4 & 5 & 4 & sehr schwach \\
\hline
\end{tabular}

\section{Datum}

1552, 9. Februar

\section{$\stackrel{*}{*} *$}

\section{Bewertung des Ereignisses}

Kein Erdbeben: Datierungsfehler.

\section{Korrektur}

1548, 9. Februar 


\section{Überlieferung}

MECOS-99

Nicht vorhanden in:

Sieberg (1940)

\section{Quelle}

Lycosthenes (1557)

\section{Interpretation}

Bei diesem Ereignis handelt es sich eindeutig um einen Abschreibefehler im MECOS-99. Denn der dort zitierte Sieberg (1940) enthält für dieses Datum keinen Eintrag, dafür einen mit identischem Tagesdatum, aber vier Jahre früher, wobei er sich auf Lycosthenes (1557) stützt. Übersetzung aus Langenbeck (1922): „1548. Am 9. Februar fand in der Nacht in Basel ein leichter Erdstoss statt, durch den Lycosthenes aus dem Schlaf erweckt wurde. Er schreibt: Ich wurde im Bett in die Höhe gehoben, würde die Erscheinung aber nicht für ein Erdbeben gehalten haben, wenn nicht verschiedene andere Personen dieselben Beobachtungen gemacht hätten. “

\section{Bemerkungen}

Vgl. das Erdbeben vom 9. Februar 1548 im SisFrance-Katalog und ECOS-09.

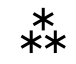

Datum

1555, 2. November
Zeit

$-$
Ort

Sondrio, I

\section{Bewertung des Ereignisses}

Kein Erdbeben, möglicherweise Bergsturz.

\section{Korrektur}

\section{Überlieferung}

- Baratta (1901)

- Rüttener (1995)

- MECOS-99

Nicht überliefert in:

- Volger (1857)

- Candreia (1905) 
- Boschi (1997)

- DOM4.1

Nicht in zeitgenössischer Zürcher Quelle:

ZBZ: Ms D 269-271. Wolfgang Haller, Tagebuch in gedrucktem Kalender 1544-1578, von Zürich

\section{Interpretation}

Dieses angebliche Erdbeben wurde mit grosser Wahrscheinlichkeit in der Schweiz nicht beobachtet und aufgezeichnet. Gemäss Baratta und einer Studie von Stucchi und Albini (1988) handelt es sich auch bei der italienischen Beobachtung eher um einen Bergsturz, da aus der Umgebung des lombardischen Val Serianas keine entsprechenden Meldungen nachweisbar sind.

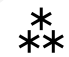

\begin{tabular}{l|ll} 
Datum & Zeit & Ort \\
1559 & - & Chur
\end{tabular}

\section{Bewertung des Ereignisses}

Kein Erdbeben.

\section{Korrektur}

\section{Überlieferung}

- Brügger (1877, S. 10): „1559. (...) Im nämlichen Jahre wurde die Stadt Chur durch ein Erdbeben erschüttert.“

- Candreia (1905)

- MECOS-99

Nicht vorhanden in:

- Campell, um 1570 (1884)

- Volger (1857)

- Baratta (1901)

\section{Interpretation}

Da Brügger (1876) seine Quelle nicht angibt und der zeitgenössische Campell (1884) diesen Hinweis nicht enthält, so muss dieser Erdbebenhinweis ohne Tagesdatierung vorläufig als Falschmeldung gewertet werden.

$$
\text { *** }
$$




\begin{tabular}{|l|l|l}
\hline Datum & Zeit & Ort \\
\hline 1569, 16. April & $8 \mathrm{Uhr}$ & Bern
\end{tabular}

\section{Bewertung des Ereignisses}

Kein Erdbeben: Datierungsfehler.

\section{Korrektur}

1569, 6. April (evt. auch 6. August)

\section{Überlieferung u. a. in:}

- Hoff (1840-1841)

- $\quad$ Volger (1857)

- MECOS-99

\section{Quelle}

- Aretius (1589)

\section{Interpretation}

Der Berner Theologe Benedikt Aretius [auch Marti genannt, um 1522-1574], hält für den 6. April 1569 Erdbebenbeobachtungen in Bern, Thun, Steffisburg, Blumenstein und mehreren dazwischenliegenden Orten fest. Seit von Hoff (1840-1841) wird auch das Datum 16. April genannt. Möglicherweise ist dieses Erdbeben sogar identisch mit dem Ereignis vom 6. August 1569.

\section{Bemerkungen}

Vgl. das Erdbeben vom 6. April 1569 im ECOS-09, das seinerseits möglicherweise identisch mit dem Erdbeben vom 6. August 1569 ist.

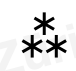
Datum
1569, 6. August
Zeit (UTC)
(4 Uhr?)
Ort
Nicht lokalisierbar (Basel)

\section{Bewertung des Ereignisses}

Sehr unsicheres Ereignis.

\section{Parameter nach ECOS}

Ix: 6 


\section{Überlieferung}

- Keckermann (1611, 3. Ed.), zitiert Aretius (1589)

- Hoff (1840-1841)

- Perrey (1845)

- Volger (1857)

- Langenbeck (1892)

- Sieberg (1940)

- SisFrance

\section{Quellen}

- Die Chronik des Fridolin Ryff 1514-1541, mit der Fortsetzung des Peter Ryff 15431585 (Vischer, 1872), Basler Chroniken, 1. Band: „Anno 1569 den 6. tag augusti morgens zwischen 5 und 6 uhren ist ein grosser erdbidem gewesen, der mit seinem heftigen erschütten vielen gebüwen schaden zugefüegt hat.“

- Aretius (1589, S. 483): „Anno 1569, die 6. Aprili levis motus concussit Bernam \& vicina loca." [Am 6. April 1569 erschütterte ein leichtes Erdbeben Bern und Umgebung.]

Kein Erdbeben-Hinweis:

ZBZ: Ms D 269-271: Wolfgang Haller, Tagebuch in gedrucktem Kalender 1544-1578, von Zürich.

\section{Interpretation}

Peter Ryff (1558-1629), der sich auf Notizen eines Unbekannten stützt, führt in seiner Chronik ein Schadenbeben in Basel am 6. August 1569 auf, das "mit seinem heftigen erschütten vielen gebüwen schaden zugefüegt hat". Der Berner Theologe Benedikt Aretius (Marti, um 1522-1574) notiert jedoch für den 6. April 1569 Erdbebenbeobachtungen in Bern, Thun, Steffisburg, Blumenstein und mehreren dazwischenliegenden Orten. Es ist unklar, ob es sich hier um zwei verschiedene Ereignisse oder um einen Fehler bei der Monatsbezeichnung handelt. Im Tagebuch von Wolfgang Haller in Zürich werden an beiden Daten Wettererscheinungen festgehalten, doch keine Erdbebenbeobachtungen. Ohne zusätzliche Quellen können somit die beiden Datierungen weder definitiv bewertet noch allenfalls miteinander verknüpft und definitiv lokalisiert werden. Seit von Hoff (1840-1841) wird auch das Datum „16. April“ genannt.

\section{Makroseismische Parameter}

\begin{tabular}{|l|l|l|l|l|l|l|}
\hline Ortschaft & Länge & Breite & $I_{\min }$ & $I_{\max }$ & $I_{\text {w }}$ & Datenqualität \\
\hline Basel & 47.56 & 7.59 & 5 & 6 & 6 & sehr schwach
\end{tabular}




\begin{tabular}{|l|l|l}
\hline Datum & Zeit (UTC) & Ort \\
\hline 1571, 19. Februar & 7 Uhr & Basel
\end{tabular}

\section{Bewertung des Ereignisses}

Sicheres Ereignis (mit unsicherer Datierung).

\section{Parameter nach ECOS}

Ix: 4

\section{Überlieferung u. a. in:}

- Gross (1624)

- Scheuchzer(1716)

- SisFrance (20. Januar 2011)

\section{Quellen}

- Wurstisen (1580)

- Chronik aus Breisach, Mone (1863): „Item im ior do man zelt hat ein thusent fynf hundert ior und sibenzeig ein ior im hornung uff den 29. (I. 28) tag zwissen 8 und 9 ist erschinen ein ertbeüdem, das die hisser zitert haben.“

- Peter Ryff (1585)

\section{Keine Hinweise in:}

- ZBZ: Ms D 269-271. Wolfgang Haller, Tagebuch in gedrucktem Kalender 15441578, von Zürich

- $\quad$ Merz (1904, S. 77-164): Werner Schodolers des Jüngern Tagebuch [Bremgarten].

\section{Interpretation}

Die Quellenlage ist widersprüchlich, denn Wurstisen datiert im Jahr 1580 auf den 19. Februar 1572, Peter Ryff im Jahr 1585 aber auf den 19. Februar 1571. Die Handschrift aus Breisach wiederum nennt das Jahr 1571, deutet aber mit der Tagesdatierung 29. Februar auf das Schaltjahr 1572. Bis weitere zeitgenössische Quellen gefunden werden, wird dieses Ereignis vorläufig auf den 19. Februar 1571 datiert, Schäden werden nirgendwo erwähnt.

\section{Makroseismische Parameter}

\begin{tabular}{|l|l|l|l|l|l|l|}
\hline Ortschaft & Länge & Breite & $I_{\min }$ & $I_{\max }$ & $I_{\text {w }}$ & Datenqualität \\
\hline Basel & 47.56 & 7.59 & 3 & 5 & 4 & sehr schwach \\
\hline Breisach, D & 48.03 & 7.58 & 3 & 5 & 4 & sehr schwach \\
\hline
\end{tabular}




\begin{tabular}{l|l|l} 
Datum & Zeit & Ort \\
$-\quad 1572,9$. Februar & -7 & - Basel \\
$-\quad 1572,19$. Februar & - & - Breisgau, D
\end{tabular}

\section{Bewertung des Ereignisses}

Keine Erdbeben: Datierungsfehler.

\section{Korrektur}

1571, 19. Februar

Überlieferung u. a. in:

- Wurstisen (1580)

- Ryff (1585)

- Mone (1863)

- Volger (1857)

\section{Interpretation}

Zum Ereignis vom 19. Februar 1571 sind unterschiedliche Fehldatierungen zu finden. So nennt zum Beispiel Wurstisen den 19. Februar 1572. Auf inn bezieht sich Volger, doch datiert er irrtümlich auf den 9. Februar 1572. In der Chronik aus Breisach (Mone, 1863) wird sogar der nichtexistierende 29. Februar 1571 genannt, was dann vom Editor auf den 28. Februar korrigiert worden ist.

\section{Bemerkungen}

Vgl. das Erdbeben vom 19. Februar 1571.

\begin{tabular}{l|l|l|} 
& \multicolumn{3}{c}{} \\
& & $* *$ \\
Datum & Zeit & Ort \\
1572, 1. Juni & - & Basel
\end{tabular}

\section{Bewertung des Ereignisses}

Kein Erdbeben: Datierungsfehler.

\section{Korrektur}

1372, 1. Juni

\section{Überlieferung u. a. in:}

- Stumpf (1548)

- Klauber (1617) 
- $\quad$ Scheuchzer (1716/17)

- $\operatorname{Volger}(1857)$

\section{Interpretation}

Klauber (1617) erwähnt als erster das angebliche Schadenbeben vom 1. Juni 1572. Seine Datierung und Formulierung („In dem Jahr hernach (1572) erzeigt sich den ersten tag Brachmonats (1. Juni) ein Erdbidem zu Basel/welcher nicht ohne sondern schrecken etliche Camin/und vom Münster S. Georgen mit anderen grossen Steinen herab warffe.") zeigt jedoch, dass er sich mit einer gewissen Wahrscheinlichkeit auf einen kompilatorischen Text für 1372 von Stumpf (1548, S. 399b) stützt: „1372 Erdbidem. Ein schwärer Erdbidem erzeigt sich abermals zuo Basel vnd umb die statt herum gar erschrockenlich. Er erschutt sich in massen/das etliche stein von dem Münster herab fielend“, dessen Originaltext von Jakob Twinger von Königshofen stammt und ein Erdbeben ohne Schäden in Strassburg am 1. Juni 1372 beschreibt. Bereits für dieses Beben existieren mehrere Datums-Varianten (vgl. 1. Juni 1372).

\section{Bemerkungen}

Vgl. das Erdbeben vom 1. Juni 1372 und die dazugehörigen Falschmeldungen.

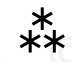

\begin{tabular}{l|l|l}
$\begin{array}{l}\text { Datum } \\
\text { Erdbebenschwarm: }\end{array}$ & Zeit (UTC) & Ort \\
-1573, 27. Mai & -18 Uhr & - Nicht lokalisierbar (Chur) \\
$-1573,4$. Juni & - mehrere & $-"$ \\
$-1573,5$. Juni & - & $-"$ \\
$-1573,6$. Juni & - & $-"$ \\
$-1573,8$. Juli & -19 Uhr & $-"$
\end{tabular}

\section{Bewertung der Ereignisse}

Unsichere bis sichere Ereignisse.

\section{Parameter nach ECOS}

Ix: (vgl. Auflistung).

\section{Überlieferung u. a. in:}

- Keferstein (1826)

- Volger (1857)

- Candreia (1905)

- MECOS-99 


\section{Quellen}

- Candreia (1905, S. 33): Darin Briefe von Pontisella in Chur an Lavater in Zürich, vom 30.6.1573, und an Ulrich Campell, I.c.II.601

- Zwei Briefe von Tobias Egli an Heinrich Bullinger, hsg. Schiess (1906, S. 427-430)

\section{Interpretation}

Es handelt sich um einen Erdbebenschwarm von Ende Mai bis mindestens anfangs Juli 1573, der in Chur und Umgebung verspürt wurde. Möglicherweise gehören noch weitere Ereignisse in diesem Jahr dazu. Als Belege dafür sind vier Dokumente vorhanden. Laut dem Brief des Rektors Pontisella vom 30. Juni ereigneten sich in Chur seit gut zwei Wochen Tag und Nacht Erdbeben, also in der zweiten Junihälfte, die mit Geräusch verbunden waren. Der ebenfalls in Chur lebende Ulrich Campell erwähnt in seiner Chronik vier einzelne Tage (27. Mai, 4. und 6. Juni und 8. Juli), wovon besonders die Nacht des 4. Juni intensiv betroffen war. Am 6. Juni war ein besonders starkes Beben in ganz Graubünden, ebenso war dasjenige vom 8. Juli stark. Gemäss den zwei Briefen von Tobias Egli, Chur, handelt es sich auch um die Nacht vom 4. auf den 5. Juni, er nennt zusätzlich noch den 6. Juni und den 8. Juli. Es gibt keinerlei Hinweise auf Schäden.

Das Erdbebendatum 30. Juni ist möglicherweise eine Verwechslung mit dem Briefdatum.

\section{Makroseismische Parameter}

27. Mai 1573:

\begin{tabular}{|l|l|l|l|l|l|l|}
\hline Ortschaft & Länge & Breite & $I_{\min }$ & $I_{\max }$ & $I_{w}$ & Datenqualität \\
\hline Chur & 46.86 & 9.53 & 3 & 4 & 4 & sehr schwach \\
\hline
\end{tabular}

4. Juni 1573:

\begin{tabular}{|l|l|l|l|l|l|l|}
\hline Ortschaft & Länge & Breite & $I_{\min }$ & $I_{\max }$ & $I_{\text {w }}$ & Datenqualität \\
\hline Chur & 46.86 & 9.53 & 5 & - & 5 & sehr schwach \\
\hline
\end{tabular}

5. Juni 1573:

\begin{tabular}{|l|l|l|l|l|l|l|}
\hline Ortschaft & Länge & Breite & $I_{\min }$ & $I_{\max }$ & $I_{w}$ & Datenqualität \\
\hline Chur & 46.86 & 9.53 & - & - & verspürt & sehr schwach \\
\hline
\end{tabular}

6. Juni 1573:

\begin{tabular}{|l|l|l|l|l|l|l|}
\hline Ortschaft & Länge & Breite & $I_{\min }$ & $I_{\max }$ & $I_{\text {w }}$ & Datenqualität \\
\hline Chur & 46.86 & 9.53 & 5 & - & 5 & sehr schwach
\end{tabular}

8. Juli 1573:

\begin{tabular}{|l|l|l|l|l|l|l|}
\hline Ortschaft & Länge & Breite & $I_{\min }$ & $I_{\max }$ & $I_{\text {w }}$ & Datenqualität \\
\hline Chur & 46.86 & 9.53 & 5 & - & verspürt & sehr schwach \\
\hline
\end{tabular}




\section{Datum \\ Zeit \\ Ort \\ 1573, 21. Dezember \\ Früher Morgen \\ Stein am Rhein, SH}

\section{Bewertung des Ereignisses}

Sicher.

\section{Parameter nach ECOS}

Ix: 5

\section{Überlieferung u. a. in:}

- Tschudi (1714)

- Bertrand (1756)

- Volger (1857)

\section{Quellen}

- Steinemann (1939), Kirchenbücher von Stein am Rhein: „(p 305) 20 decembris [1573] was sunntag, da kam zu nacht nach mitternacht als es gegen den 21 tag was vor dem einen, ein grosser Erdbidem dies man zu Zürich, Schaffhausen, Stein und Stoken und darumb gewar ward. Gott syge uns gnedig. “

- StASH, ohne Signatur: Chronik der Stadt Schaffhausen, Spleiss, ungefähr 1570 entstanden.

Keine Hinweise in den zeitgenössischen Notizen von:

- ZBZ: Ms D 269-271. Wolfgang Haller, Tagebuch in gedrucktem Kalender 15441578, von Zürich

- Vischer (1872): Die Chronik des Fridolin Ryff 1514-1541, mit der Fortsetzung des Peter Ryff 1543-1585

\section{Interpretation}

Die Datierung 21. Dezember 1573 ist mit den zeitgenössischen Beobachtungen aus Stein am Rhein (21.12.) und Schaffhausen (20.12.) sowie der Kopie aus Glarus (21.12.) zuverlässig gesichert. Die Intensität wird mit „gross“ und „stark“ umschrieben, die zeitgenössischen Quellen, bzw. die Tschudi-Kopie für Glarus erwähnen aber keine Schäden.

Erst bei Bertrand (1756) taucht ohne Quellenbeleg „quelque dommage à Glaris“ auf, eine Aussage, die ohne zeitgenössische Bestätigung nicht in die Wertung einbezogen werden kann; fragwürdig sind die Hinweise auf Luzern und Neuchâtel. 
Makroseismische Parameter

\begin{tabular}{|l|l|l|l|l|l|l|}
\hline Ortschaft & Länge & Breite & $I_{\min }$ & $I_{\max }$ & $I_{\text {w }}$ & Datenqualität \\
\hline Stockach & 47.85 & 9.01 & & & verspürt & sehr schwach \\
\hline Glarus & 47.04 & 9.07 & & & verspürt & sehr schwach \\
\hline Neuchâtel & 46.99 & 6.93 & & & verspürt & sehr schwach \\
\hline Luzern & 47.05 & 8.29 & & & verspürt & sehr schwach \\
\hline Stein am Rhein & 47.66 & 8.86 & 4 & 5 & 5 & sehr schwach \\
\hline Schaffhausen & 47.7 & 8.64 & 3 & 5 & 4 & sehr schwach \\
\hline Zürich & 47.37 & 8.54 & 2 & 4 & 3 & sehr schwach \\
\hline
\end{tabular}

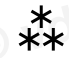

\section{Datum}

- 1573, 20. Dezember

- 1573, 21. Dezember

\section{Zeit}

Ort

- Klöntal, GL

- Glarus

\section{Bewertung des Ereignisses}

Keine Erdbeben: Lokalisierungsfehler.

\section{Korrektur}

Stein am Rhein

\section{Überlieferung $u$. a. in:}

- Tschudi (1714)

- Bertrand (1756)

- Volger (1857)

- Milne (1899)

- Montandon (1942-43)

- MECOS-99

\section{Interpretation}

Die angeblichen Schäden in Glarus lassen sich in Tschudis Beschreibung noch nicht finden (21. Dezember 1573: An St. Thomas Abend ist ein starcker Erdbidem in dem Land Glarus vermercket worden), sie tauchen erstmals bei Bertrand auf mit "quelque dommage à Glaris“. Deshalb wurde das Epizentrum Stein am Rhein zugeordnet, vgl. oben. 


\section{Bemerkungen}

Vgl. im ECOS-09 das Erdbeben vom 21. Dezember 1573 in Stein am Rhein.

\begin{tabular}{|c|c|c|}
\hline $\begin{array}{l}\text { Datum } \\
\text { 1574, 3. Mai }\end{array}$ & $\begin{array}{l}\text { Zeit } \\
-\end{array}$ & $\begin{array}{l}\text { Ort } \\
\text { Genève }\end{array}$ \\
\hline
\end{tabular}

\section{Bewertung des Ereignisses}

Kein Erdbeben: Datierungsfehler.

\section{Korrekturvorschlag}

Eventuell am 11. März 1584.

\section{Überlieferung u. a. in:}

- $\quad$ AEGE: Ms hist. no 19 (ancien 141bis), Annales de Genève 1603-1766

- $\quad$ Spon (1680/82)

- Bertrand (1756)

- Volger (1857)

\section{Interpretation}

Es sind keine zeitgenössischen Beschreibungen für ein Erdbeben zu diesem Datum gefunden worden. Erst bei Spon (1680/82) steht neben diesem Datum auch eine Schadensbeschreibung: „renversa dans le fossé la Porte de Cornevin“ [Stürzte das Tor Cornevin in den Graben]. In den „Annales de Genève“ aus dem 18. Jahrhundert werden für den 3. Mai 1574 zwei Erdbeben festgehalten, aber ohne allfällige Schäden zu erwähnen.

Da beide Angaben aus Kopien stammen, stellt sich die Frage, ob es sich hier nicht um eine Verwechslung mit den Schadenbeben vom März 1584 handelt, wofür es sogar eine ähnliche Falschdatierung gibt, nämlich 3. Mai 1584, die aus der gedruckten Chronik von Michael Stettler (1626-27) stammt und Spon bekannt sein konnte. Mit der vorliegenden Quellenlage kann dies aber noch nicht eindeutig beantwortet werden, denn für den 11.-14. März 1584 (1.-4. März, jul. Kalender) sind nur geringe Schäden in Genf überliefert worden. Da aber ein derart grosser Schaden zu diesem Zeitpunkt bereits deutlichere Spuren in den Quellen hinterlassen müsste, ist vorläufig von einer Falschmeldung auszugehen.

\section{Bemerkungen}

Vgl. das Erdbeben vom 11. März 1584.

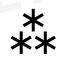




\begin{tabular}{|l|l|l} 
Datum & Zeit & Ort \\
\hline 1574, 30. Juni & - & Zürich
\end{tabular}

\section{Bewertung des Ereignisses}

Kein Erdbeben: Datierungsfehler.

\section{Korrektur}

1574, 30. Juli

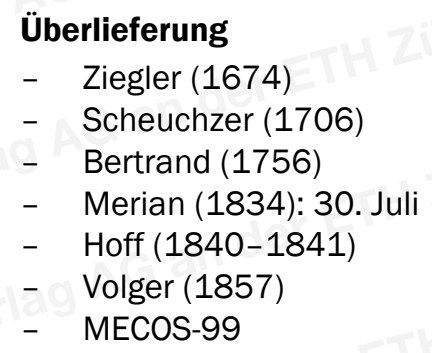

Datum in zeitgenössischen Quellen:

- StAZH: E III 062.1, Seite 215; Kilchberg, Pfarrbuch 1536-1617: „1574. Dornach den 30. Julij erhub sich inn der statt, am seew und anderen umbligenden orten, wie ouch ze baden und zug eyn starker Erdbidem, nach undergang der sonnen."

- Vischer (1872, S. 227), Ryff, Peter und Fridolin: „Erdbidem. Anno 1574 uff frytag den 30. Julii zu nacht zwüschen 9 undt zechen uhren ist ein schrecklicher erdbidem zu Basell undt umbligenden landtschaft gewesen, die gebüw heftig erschuttert, doch ohne nochteil baldt wider nachgelassen."

- StASH o. S.: Chronik der Stadt Schaffhausen, zusammengetragen und geschrieben durch Hans Jacob Spleiss, V. D. M. [Pfarrer, 1586-1657]. „1574. [...] Den 30. Julij ist ein kleiner Erdbidem gewesen."

- ZBZ: Ms F 23.357, Wickiana. „1574, 30. Juli, Zürich: Von einem erdbidem. Am 30 Julij um die 8 uhren nach mittag zu angender nacht, haben vil lüth einen erdbidem gehört, darab vil hüser erzitteret, was es bedüte, weise Gott.“

\section{Interpretation}

Die Datierung 30. Juni ist zum ersten Mal in Ziegler (1674) zu finden: „Anno 1574. Den 30. Brachmonat (Juni)/war ein gar starker Erdbidem. “ Und später ebenso in Scheuchzer (1706): „A. 1574. den 30. Jun. erzeigte sich zu Zürich ein Erdbeben. ex Msc. Antiqu. Bibl. Tig. n. 52." Sie beruht eindeutig auf einer Verwechslung von Juni mit Juli, wie die zeitgenössischen Quellen belegen. 


\section{Bemerkungen}

Vgl. im ECOS-09 das Erdbeben vom 30. Juli 1574, lokalisiert in Zürich.

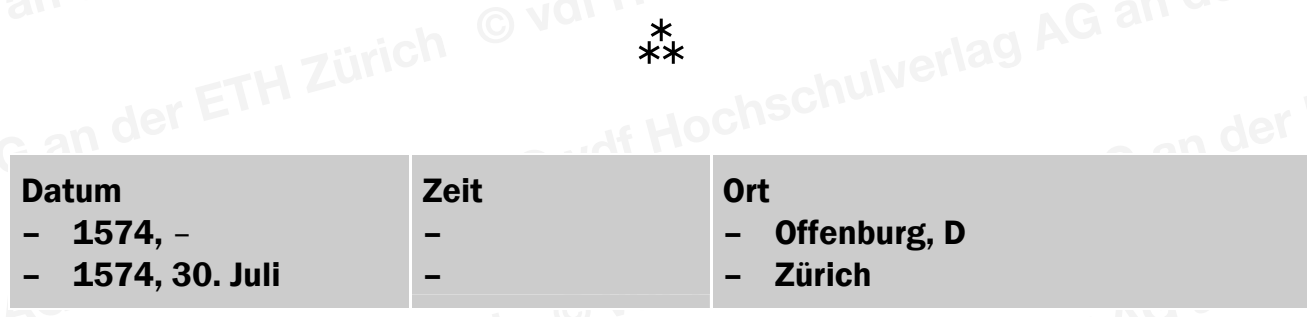

\section{Bewertung des Ereignisses}

Unsicheres und sicheres Erdbeben.

\section{Parameter nach ECOS}

- 1574, Offenburg

- Ix:-

- $\quad$ 30. Juli 1574, Zürich

- Ix: 4

\section{Überlieferung u. a. in:}

- Beuther (1601): „1574. Anno Christi 1574. Ist ein starcker Erdbidem zu Offenburg gewesen/durch welchen ein grosser theil der Stadtmauren eingefallen.“

- $\quad$ Hoff (1840-1841)

- Langenbeck (1892)

- Giessberger (1922)

- Sieberg (1940)

- Gils, Van \& Leydecker (1991)

- Rüttener (1995)

- MECOS-99

- EKDAG 2006

- SisFrance (1. September 2009)

\section{Quellen}

Zeitgenössische Schweizer Quellen für den 30. Juli 1574:

- $\quad$ Ryff (Vischer, 1872, S. 227): „Erdbidem. Anno 1574 uff frytag den 30. Julii zu nacht zwüschen 9 undt zechen uhren ist ein schrecklicher erdbidem zu Basell undt umbligenden landtschaft gewesen, die gebüw heftig erschuttert, doch ohne nochteil baldt wider nachgelassen."

- StAZH: E III 062.1, Seite 215, Pfarrbuch Kilchberg: „1574 (...) dornach den .30. Julij erhub sich inn der statt, am seew und anderen umbligenden orten, wie ouch ze baden und zug eyn starker Erdbidem, nach undergang der sonnen.“ 
- StASH o. S.: Chronik der Stadt Schaffhausen, zusammengetragen und geschrieben durch Hans Jacob Spleiss, V. D. M. [Pfarrer, 1586-1657]. „1574. [...] Den 30. Julij ist ein kleiner Erdbidem gewesen."

- ZBZ: Ms F 23.357, Wickiana. „1574, 30. Juli, Zürich: Von einem erdbidem. Am 30 Julij um die 8 uhren nach mittag zu angender nacht, haben vil lüth einen erdbidem gehört, darab vil hüser erzitteret, was es bedüte, weise Gott.“

\section{Interpretation}

Beuthers Meldung aus Offenburg (ungefähr 100 km nördlich von Basel) konnte im Rahmen dieses SED-Projektes nicht weiter untersucht werden. Zur Zeit des Erdbebens hat der Verfasser aber im nahegelegenen Strassburg gelebt, sodass die Beschreibung nicht ganz unglaubwürdig zu sein scheint.

Da Beuther keine Tagesdatierung für das Schadenbeben in Offenburg gibt, so ist bei künftigen Untersuchungen die Frage zu stellen, ob es zu den gesicherten Schweizer Erdbebenmeldungen aus Basel, Zürich, Baden und Zug vom 30. Juli gehört.

\section{Makroseismische Parameter}

Offenburg 1574

\begin{tabular}{|l|l|l|l|l|l|l|}
\hline Ortschaft & Länge & Breite & $I_{\min }$ & $I_{\max }$ & $I_{w}$ & Datenqualität \\
\hline Offenburg & 48.48 & 7.96 & 6 & 7 & & sehr schwach \\
\hline
\end{tabular}

Makroseismische Parameter aus der Schweiz für den 30. Juli 1574, Zürich

\begin{tabular}{|l|l|l|l|l|l|l|}
\hline Ortschaft & Länge & Breite & $I_{\min }$ & $I_{\max }$ & $I_{\text {w }}$ & Datenqualität \\
\hline Basel & 47.56 & 7.59 & 3 & 5 & 4 & schwach \\
\hline Baden & 47.47 & 8.31 & 4 & 5 & 4 & sehr schwach \\
\hline Kilchberg ZH & 47.32 & 8.54 & 3 & 5 & 4 & sehr schwach \\
\hline Zürich & 47.37 & 8.54 & 3 & 5 & 4 & sehr schwach \\
\hline Zug & 47.17 & 8.52 & 4 & 5 & 4 & sehr schwach \\
\hline Schaffhausen & 47.7 & 8.64 & 3 & 4 & 3 & sehr schwach \\
\hline
\end{tabular}

\begin{tabular}{|l|l|l} 
Datum & Zeit & Ort \\
\hline 1577, 22. September & - & Nicht lokalisierbar (Bern; Colmar, F)
\end{tabular}

\section{Bewertung des Ereignisses}

Sicheres Ereignis. 


\section{Parameter nach ECOS}

Ix: 5

\section{Überlieferung}

- StASH, ohne Signatur: Spleiss (1586-1657)

- Rasch (1591), Wien

- Gross (1624), Basel

- Tschamser Chronik (1864), Thann

\section{Quellen}

- Ragor (1578)

- $\quad$ Cysat (1969, Tafel 34, S. 702) überliefert ein Flugblatt von Apiarius (Basler Druckerei) und kommentiert es teilweise.

\section{Interpretation}

Ende September 1577 haben im Raum Schaffhausen-Colmar-Basel-Bern mehrere Beben stattgefunden, wovon der Hauptstoss mit grosser Wahrscheinlichkeit am 22. September geschah, möglicherweise gefolgt von zwei Nachbeben am selben Tag (Gross, 1624) und gemäss Ragor (1578) von weiteren Nachbeben am 23., 24., 25. und 29. September. Das in der Cysat-Ausgabe mitpublizierte Flugblatt (Tafel 34, S. 702) von Apiarius, Basel, enthält mit der Datierung „xviij Herbstmonat“ (18. Sept.) wahrscheinlich eine Fehldatierung. Allerdings kommentiert Cysat die darin erwähnten Erdbebenschäden nicht. Flugblätter, die es es seit dem 15. Jahrhundert gibt, waren ein kommerziell geprägtes und massenwirksames Kommunikationsmittel; somit als Quelle wenig zuverlässig.

Das Flugblatt fasst fünf angebliche Wunderzeichen des Jahres 1577 in unpräziser Formulierung zusammen und illustriert sie, so auch Erdbeben: „Hiemit lauffen auch vnder/ die grosse hefftige Erbidem/die man mit grosser forcht vnd erschrecke diss jar her empfunden vnd gehört/insonderheit aber hat man dieselben vom xviij (!) tag Herbstmonat her [18. September]/von Colmar an biss gehn Bern in die zwentzig meil wegs mercklich gespürt/also/das nu an etlichen orten die gebeuw vnd heuser dermassen zerrütt vnd zerschelt/dz grosser rissz vn spelt daran gesehen werden.“ Der zeitgenössische Ragor, der Hilfsprediger im nahe bei Bern liegenden Herzogenbuchsee war, notiert hingegen ausdrücklich „keine Schäden in Basel und Bern“. Die wenig verlässliche Tschamser Chronik von Thann im Elsass wiederum spricht lediglich von kleinen Schäden (S. 208): 1577: „Den 22. und 23. Octobris hört und spürte man von hier bis gen Basel hinauf abermahl einen ziemlichen grossen Erdbidem, welcher jedoch nicht gar vil geschadet.“

Da alle Wunderzeichen vom Flugblatt reisserisch dargestellt sind und das Beben nur ungenau lokalisiert ist, müssen die Schäden bis zur Verifizierung durch weitere Quellen, als zu unsicher gewertet werden. Ortsbezeichnungen wie Frutigen tauchen in den Kompilationen erst im 18. Jahrhundert auf und könnten sich auch auf andere Beben im Jahre 1577 beziehen, wie zum Beispiel auf dasjenige im Februar. 


\section{Makroseismische Parameter}

\begin{tabular}{|c|c|c|c|c|c|c|}
\hline Ortschaft & Länge & Breite & \multicolumn{2}{|c|}{$I_{\min }$} & $I_{w}$ & Datenqualität \\
\hline Basel & 47.56 & 7.59 & & & verspürt & sehr schwach \\
\hline Aigle & 46.32 & 6.97 & & & verspürt & sehr schwach \\
\hline Bern & 46.98 & 7.46 & 4 & 5 & 5 & sehr schwach \\
\hline Schaffhausen & 47.7 & 8.64 & 3 & 4 & 3 & sehr schwach \\
\hline Colmar, F & 48.09 & 7.37 & - & - & verspürt & sehr schwach \\
\hline $\begin{array}{l}\text { Datum } \\
-\quad 1584,11 . \text { März } \\
-\quad 1584,14 . \text { März }\end{array}$ & & $\begin{array}{l}\text { TC) } \\
\text { Uhr } 30 \\
\text { Uhr }\end{array}$ & & $\begin{array}{l}\text { Ort } \\
\text { - Aigle, } \\
\text { - Aigle, }\end{array}$ & & \\
\hline
\end{tabular}

\section{Bewertung der Ereignisse}

Sichere Ereignisse.

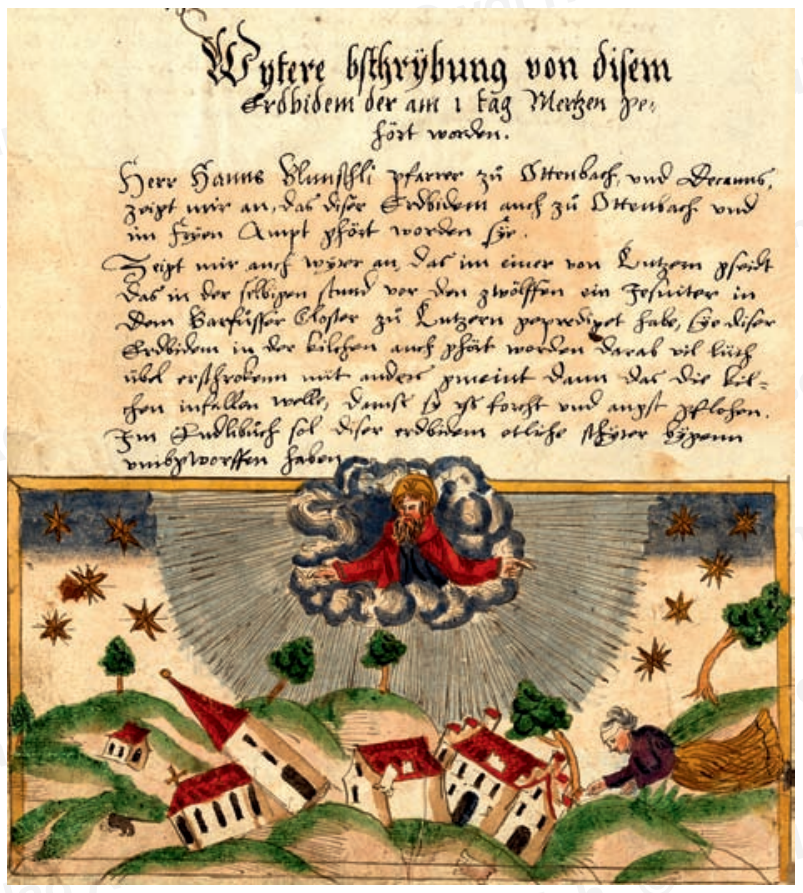

Abbildung 6: Zeitgenössische fiktive Darstellung des Erdbebens vom 11. (1.) März 1584 in Aigle, VD, in der Nachrichtensammlung Wickiana. Die religiöse Interpretation der seismischen Vorgänge ist klar erkennbar (ZBZ, Wickiana, Ms F 32, folio 19-22). 


\section{Parameter nach ECOS}

- Ix: 8 (11. März, Hauptstoss)

- Ix: 7 (14. März, Nachbeben)

\section{Publiziert in:}

- Schwarz-Zanetti (in prep., 2011)

- Schwarz-Zanetti (2008e)

\section{Zusammenfassung}

Das Gebiet des Genfer Sees wurde vom 11.-14. März 1584 von einer Erdbebenserie erschüttert, deren erster Stoss nicht nur Gebäude beschädigte, sondern mit einer vermuteten subaquatischen Rutschung im Rhonedelta auch die Uferpartien und das Schloss Chillon stark beschädigte. Gleichzeitig löste sich ein Bergsturz von der Tour d'Aï, der für drei Tage auf der Terrasse von En-Luan liegenblieb und sich danach in Form eines Schuttstroms verheerend über die Dörfer Corbeyrier und Yvornaz ergoss, wobei 122 Einwohner und 206 Fremde getötet worden sein sollen.

\section{Makroseismische Parameter}

11. März

\begin{tabular}{|l|l|l|l|l|l|l|}
\hline Ortschaft & Länge & Breite & $I_{\min }$ & $I_{\max }$ & $I_{\text {w }}$ & Datenqualität \\
\hline Montreux, VD25 & 46.43 & 6.91 & 7 & 8 & 8 & schwach \\
\hline Bex, VD & 46.25 & 7.01 & 7 & 8 & 8 & sehr schwach \\
\hline Aigle, VD & 46.32 & 6.97 & 6 & 7 & 7 & gut \\
\hline Lausanne & 46.52 & 6.63 & 6 & 7 & 7 & schwach \\
\hline Glion, VD26 & 46.43 & 6.93 & 6 & 7 & 7 & sehr schwach \\
\hline Les Ormonts, VD & 46.35 & 7.11 & 6 & 7 & 7 & sehr schwach \\
\hline Corbeyrier, VD & 46.35 & 6.96 & 6 & - & 7 & schwach \\
\hline Vevey, VD & 46.46 & 6.84 & 6 & 7 & 7 & schwach \\
\hline Murten, FR & 46.93 & 7.13 & 6 & 7 & 6 & schwach \\
\hline Genève & 46.21 & 6.14 & 6 & 7 & 6 & schwach \\
\hline Villeneuve, VD & 46.4 & 6.96 & 6 & 7 & 6 & schwach \\
\hline St-Maurice, VS & 46.22 & 7 & 6 & 7 & 6 & sehr schwach \\
\hline Noville, VD & 46.38 & 6.90 & 5 & 7 & 6 & sehr schwach \\
\hline & & & & & \\
\hline
\end{tabular}

\footnotetext{
25 Umfasst auch das Schloss Chillon.

26 Die Lokalisierung „Lyon“ in den Quellen wird hier „Glion“ zugeordnet (evt. auch Leysin).
} 


\begin{tabular}{|l|l|l|l|l|l|l|}
\hline Ortschaft & Länge & Breite & Imin & Imax & Iw & Datenqualität \\
\hline Suchy, VD & 46.72 & 6.6 & 5 & 6 & 6 & sehr schwach \\
\hline Luzern & 47.05 & 8.29 & 5 & 6 & 6 & schwach \\
\hline Yvorne, VD & 46.33 & 6.96 & 6 & - & - & sehr schwach \\
\hline Kanton Glarus & 46.98 & 9.06 & 5 & 6 & 5 & sehr schwach \\
\hline Stein/Rhein, SH & 47.66 & 8.86 & 4 & 6 & 5 & sehr schwach \\
\hline Grandvaux, VD & 46.5 & 6.73 & 4 & 6 & 5 & sehr schwach \\
\hline Marboz, F & 46.35 & 5.25 & 4 & 6 & 5 & sehr schwach \\
\hline Aarberg, BE & 47.04 & 7.28 & 4 & 5 & 5 & sehr schwach \\
\hline Kilchberg, ZH & 47.32 & 8.54 & 4 & 5 & 5 & sehr schwach \\
\hline Rougemont, VD & 46.49 & 7.21 & 4 & - & 5 & sehr schwach \\
\hline Saanen, BE & 46.49 & 7.26 & 4 & - & 5 & sehr schwach \\
\hline Biel, BE & 47.14 & 7.25 & 4 & 5 & 5 & sehr schwach \\
\hline Entlebuch, LU & 46.99 & 8.06 & 4 & 5 & 5 & sehr schwach \\
\hline Annecy, F & 45.91 & 6.13 & - & - & 5 & schwach \\
\hline Zürich & 47.37 & 8.54 & 4 & 5 & 4 & schwach \\
\hline Duingt, F & 45.83 & 6.20 & - & - & verspürt & sehr schwach \\
\hline Talloires, F & 45.83 & 6.22 & - & - & verspürt & sehr schwach \\
\hline Saint-Jorioz, F & 45.83 & 6.16 & - & - & verspürt & sehr schwach \\
\hline Kanton Uri & 46.76 & 8.68 & - & - & verspürt & sehr schwach \\
\hline Ottenbach, ZH & 47.28 & 8.41 & - & - & verspürt & sehr schwach \\
\hline Kanton Wallis & 46.26 & 7.62 & - & - & verspürt & sehr schwach \\
\hline Piemont, I & 45.25 & 7.67 & - & - & verspürt & sehr schwach \\
\hline Saint Antoine, F & 45.17 & 5.22 & - & - & verspürt & sehr schwach \\
\hline Bodensee & 47.57 & 9.43 & - & - & verspürt & sehr schwach \\
\hline Kanton Schwyz & 47.06 & 8.69 & - & - & verspürt & sehr schwach \\
\hline Kanton Zug & 47.16 & 8.55 & - & - & verspürt & sehr schwach \\
\hline Kanton Nidwalden & 46.9 & 8.4 & - & - & verspürt & sehr schwach \\
\hline Boswil, AG & 47.3 & 8.31 & - & - & verspürt & sehr schwach \\
\hline Maienfeld, GR & 47.01 & 9.53 & 1 & 2 & 1 & sehr schwach \\
\hline & & & & & \\
\hline
\end{tabular}


14. März

\begin{tabular}{|l|l|l|l|l|l|l|}
\hline Ortschaft & Länge & Breite & $I_{\min }$ & $I_{\max }$ & $I_{\text {w }}$ & Datenqualität \\
\hline Genève & 46.21 & 6.14 & & & verspürt & sehr schwach \\
\hline Montreux & 46.43 & 6.91 & 6 & & verspürt & sehr schwach \\
\hline Yvorne & 46.33 & 6.96 & 5 & & verspürt & sehr schwach \\
\hline Corbeyrier & 46.35 & 6.96 & 7 & & verspürt & sehr schwach \\
\hline Neuchâtel & 46.99 & 6.93 & 3 & 5 & verspürt & sehr schwach \\
\hline Aigle & 46.32 & 6.97 & 7 & 7 & 7 & sehr schwach \\
\hline Vevey & 46.46 & 6.84 & 6 & 7 & 6 & sehr schwach \\
\hline Villeneuve VD & 46.4 & 6.96 & 5 & 6 & 6 & sehr schwach \\
\hline
\end{tabular}

\section{Bemerkungen}

Vgl. im ECOS-09 die Erdbeben vom 11.-14. März 1584.

Vgl. die limnogeologisch nachgewiesenen subaquatischen Rutschungen im Vierwaldstättersee nach dem Erdbeben in Unterwalden vom 18. September 1601.

Bei zwei weiteren Überschwemmungen lassen sich subaquatische Rutschungen vermuten, wobei aber keine vorangehende Erdbeben nachgewiesen sind. So könnte es sich bei der möglichen Überschwemmung vom 11. September 1666 in Arbon, TG, am Bodensee ebenfalls um eine subaquatische Rutschung gehandelt haben; v.a. da der Sommer 1666 ausgesprochen trocken war. Andererseits ist die Überschwemmung von Brunnen am Vierwaldstättersee vom 24. September 1687 zuverlässig dokumentiert, doch auch da kann kein vorausgehendes Erdbeben belegt werden. Vielmehr ging diesem Abbruch des Muotadeltas eine längere Niederschlagsperiode voraus.

Es sind auch noch Schäden an der Kathedrale von Lausanne zu erwähnen, die vermutlich irrtümlicherweise auf das Jahr 1655 datiert wurden.

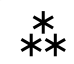

\begin{tabular}{|c|c|c|}
\hline 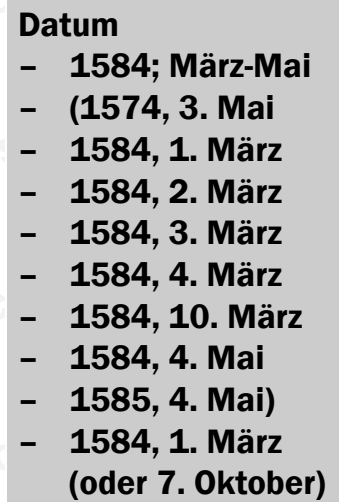 & $\begin{array}{l}\text { Zeit } \\
-\end{array}$ & $\begin{array}{ll} & \\
\text { - Basel } \\
\text { - Dronero, I }\end{array}$ \\
\hline
\end{tabular}




\section{Bewertung des Ereignisses}

Keine Erdbeben: Datierungsfehler.

\section{Korrektur}

1584, 11.-14. März

\section{Überlieferung u. a. in:}

- Stettler (1626 und 1627)

- Bertrand (1756)

- $\quad$ olger (1857)

- Barrata (1901)

- Candreia (1905)

- Montandon (1942-43)

\section{Interpretation}

Bei den oben aufgeführten Daten vom 1.-4. März 1584 handelt es sich um die damals noch in einigen katholischen und in den reformierten Kantonen der Schweiz verbreitet gebräuchliche julianische Datierungen der Ereignisse vom 11.-14. März 1584. Da einige katholische Kantone der Schweiz bereits im Januar 1584 den vom Papst initiierten Kalenderwechsel vom Oktober 1582 nachvollzogen hatten, werden im Schweizerischen Erdbebenkatalog (ECOS-02 und ECOS-09) zwecks Datierungs-Harmonisierung alle Beben ab 1584 im gregorianischen Kalenderstil datiert.

Das Mai-Datum ist ein Druckfehler aus der Chronik von Michael Stettler (1626 und 1627), wobei wahrscheinlich das julianische März-Datum mit dem Mai verwechselt worden ist. Die Datierung 1574 stammt aus der Chronik von Spon (1680) und bezieht sich möglicherweise auch auf die März-Ereignisse von 1584.

Es ist sehr unwahrscheinlich, dass das Schadenbeben vom 7. Oktober 1584 in Dronero, I, zitiert in Baratta (1901), sich auch auf das Beben vom 11. März 1584 bezieht, denn Dronero befindet sich ungefähr $200 \mathrm{~km}$ südlich des Epizentrums, auch wenn der italienische Katalog DOM4.1 es auf den 1. März datiert.

\section{Bemerkungen}

Vgl. im ECOS-09 die Erdbeben vom 11.-14. März 1584.

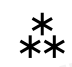

\begin{tabular}{|l|l|l} 
Datum & Zeit (UTC) & Ort \\
\hline 1588, 11. Juni & - & Singen, D
\end{tabular}

\section{Bewertung des Ereignisses}

Kein Erdbeben: Datierungsfehler.

\section{Korrektur}

1588, 21. Juni 


\section{Überlieferung}

- Langenbeck (1892)

- Sieberg (1940)

- MECOS-99

- EKDAG 2006

\section{Interpretation}

In Sieberg (1940) wird dieses Beben auf den 11. und 12. Juni 1588 datiert, er beruft sich dabei auf das Manuskript von Lersch (GFZ; wurde nicht eingesehen) und auf Giessberger (1922). Langenbeck (1892) datiert aber zehn Tage später, also auf den 21. und 22. Juni. Damit kann es sich um einen Kalenderfehler handeln, wegen fehlender Augenzeugenberichte ist aber eine Falschmeldung ebenso wahrscheinlich.

\section{Bemerkungen}

Vgl. im ECOS-09 den Eintrag zum 21. Juni 1588.

\section{**}

\begin{tabular}{|l|l|l}
\hline Datum & Zeit (UTC) & Ort \\
\hline 1588, 21. Juni & - & Nicht lokalisierbar (Singen, D)
\end{tabular}

\section{Bewertung des Ereignisses}

Sehr unsicheres Ereignis.

\section{Parameter nach ECOS}

Ix: 6

\section{Überlieferung u. a. in:}

- Tschamser (1864)

- Langenbeck (1892, S. 365)

- Sieberg (1940)

- MECOS-99

\section{Quellen}

Nicht in zeitgenössischer Quelle:

- StASaR: Kirchenbücher 1559-1638, Ki 1

\section{Interpretation}

Tschamser (1864), eine im Allgemeinen unzuverlässige Kompilation, wird von Langenbeck (1892, S. 365) zitiert: „Am 21. und 22. Juni wurde in der Umgebung des Hohen Twiel ein starkes Erdbeben gespürt, das jedoch keinen sonderlichen Schaden anrichtete, ausser dass es einige Schornsteine umwarf." Es ist nur Sieberg (1940), der auf den 11. und 12. Juni datiert; möglicherweise handelt es sich hier um eine julianische Datierung. 
Die für Erdbeben sensibilisierten Kirchenbücher des nahen Stein am Rhein, SH, erwähnen an diesem Datum kein Erdbeben. Ohne zeitgenössische Beobachtungen muss dieses Beben in Singen, wenige Kilometer von der Nordgrenze der Schweiz entfernt, als „sehr unsicher“ gewertet werden. Als mögliches Schadenbeben sollte dieses Datum jedoch bei einer künftigen Quellensuche im Auge behalten werden.

\section{Makroseismische Parameter}

\begin{tabular}{|l|l|l|l|l|l|l|}
\hline Ortschaft & Länge & Breite & $I_{\min }$ & $I_{\max }$ & $I_{w}$ & Datenqualität \\
\hline Singen & 47.76 & 8.86 & 6 & 7 & 6 & sehr schwach \\
\hline
\end{tabular}

\section{***}

\section{Datum}

1593, 6. Februar

\section{Zeit}

$-$
Ort

Nördlingen, D

\section{Bewertung des Ereignisses}

Kein Erdbeben: Sturm.

\section{Überlieferung u. a. in:}

- Der möglicherweise älteste kompilatorische Hinweis konnte im Rahmen dieses Projektes nicht überprüft werden: Müller (1824): „Merkwürdigkeiten der Stadt Nördlingen nebst einer Chronik mit lythographischen Zeichnungen“, die sich gemäss Giessberger (1922) auf die nicht eruierbare Wenig'sche Chronik stützt.

- Giessberger (1922): „Den 16. Februar (1593) ist hier (Nördlingen) ein grosses Erdbeben 2 Stunden lang verspühret worden, welches viele Camine und Dächer herabgeworffen, auch Tannen und Linden nebst anderen Bäumen mitsambt den Wurtzen aus dem Boden und darnieder gerissen, folglich grossen Schaden angerichtet hat.“

- Sieberg (1940)

- Grünthal (2001, S. 21)

Nicht vorhanden in:

- Volger (1857)

- Candreia (1905)

- Climhist (1984)

- SisFrance (2009)

\section{Quellen}

Nicht vorhanden in:

- Kirchenbüchern von Stein am Rhein (StASaR: Ki 1)

- Wetternotizen von Cysat (1969) 


\section{Interpretation}

Im Rahmen des vorliegenden Projektes konnte in den Schweizer Quellen und Kompilationen weder ein Erdbeben noch eine allfällige Verwechslung mit einem Sturm nachgewiesen werden. Grünthal hält nur aufgrund der Beschreibung in Giessberger eine Verwechslung mit einem Sturm für gesichert, sodass dieses Ereignis vorläufig als Falschmeldung gewertet wird.

\section{Bemerkungen}

Vgl. die Verwechslung eines Erdbebens mit einem Sturm am 26. Juni 1517.

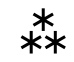

\section{Datum}

- 1593, Okt. - 1594, Nov.

- 1593, 10. Oktober

- 1593, 10. November

- 1593, 11. November

- 1593, 21. November

- 1594, März

- 1594, 11. November
Ort

Glarus - Klöntal

\section{Bewertung des Ereignisses}

Keine Erdbeben: Bergstürze von 1593-1594.

Überlieferung $\mathbf{u}$. a. in:

Kompilationen:

- Tschudi, J. (1714)

- Scheuchzer(1716)

- Trümpi (1774)

- Volger (1857)

- Tschudi, N. (1869)

- Gils, Van \& Leydecker (1991)

- MECOS-99

Nicht vorhanden in:

- Candreia (1905)

Zeitgenössische Quellen:

- Sprecher (1992): Aus Bartholomäus Anhorns Chronik der Stadt Maienfeld

- $\quad$ Ardüser (1877): Rätische Chronik 


\section{Interpretation}

Wahrscheinlich zwischen dem November 1593 und dem Ende des Jahres 1594 fielen ein oder mehrere Bergstürze vom Glärnisch herab. Die Aussage, dass am 21. November 1593 angeblich ein Erdbeben einen ersten Bergsturz vom Glärnisch ausgelöst habe, ist zwar zumindest bis auf Johann Heinrich Tschudi (1714) zurückzuführen. Doch der zeitgenössische Bartholomäus Anhorn, Pfarrer und aufmerksamer Chronist auch für Naturphänomene im 35 km entfernten Maienfeld, erwähnt dieses angebliche Erdbeben nicht, erst für den Frühling 1594 hält er ein grosses Bergsturz-Ereignis ohne Erdbebenhinweis bei Glarus fest. Auch beim Bündner Chronisten Hans Ardüser (1557- ungef. 1617), der ebenso aufmerksam Naturbeobachtungen festhält, findet sich kein Hinweis auf 1593, erst für das Jahr 1594 ist ein Bergsturzereignis bei Glarus erwähnt, aber ohne Tagesangabe, auch hier fehlt ein Erdbebenhinweis. Allerdings wird der Bergsturz bei ihm, vielleicht zufällig, erst nach dem 13. Oktober aufgeführt. Diese Bergstürze fehlen beim Bündner Erdbeben-Kompilatoren Candreia (1905) gänzlich. Sodass die Vermutung nahe liegt, dass der Glarner Kompilator J. H. Tschudi (1714) und seine unbekannten Vorlagen die Erschütterungen durch die Bergstürze mit einem Erdbeben verwechselt haben können.

Rund um die Glarner Bergstürze werden unterschiedliche Datierungen genannt. Die Oktober-1593-Datierung taucht erstmals in Van Gils \& Leydecker (1991) auf und muss als Druckfehler gewertet werden. Die Tagesdatierungen 10. oder 11. November beziehen sich auf den julianischen Kalender, zu denen zehn Tage addiert werden müssten. Sie gehen wahrscheinlich auf die Angaben in der Chronik von J. H. Tschudi (1714) zurück. Niklaus Tschudi (1869) nennt in seiner Untersuchung eine Serie von Bergstürzen vom 21. (11.) November 1593 bis in den Juli 1594. Ardüsers Datierung erlaubt sogar eine Ausdehnung bis zum Jahresende.

Damit kann ein Erdbeben in diesem Zeitbereich mit einer gewissen Wahrscheinlichkeit ausgeschlossen werden, für eine genauere Datierung der Bergstürze wären zusätzliche Dokumente erforderlich.

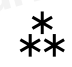

\begin{tabular}{|l|l|l}
\hline Datum & Zeit & Ort \\
\hline $1593,5$. November & - & Neuchâtel
\end{tabular}

\section{Bewertung des Ereignisses}

Kein Erdbeben: Datierungsfehler.

\section{Korrektur}

1593, 15. November

\section{Überlieferung u. a. in:}

- Bertrand (1756, S. 250): „Le cinquième Novembre, mille cinq-cent quatre-vingt-treize, on sentit un tremblement de terre à Neufchâtel, \& en divers autres lieux voisins.“

- $\operatorname{Volger}(1857)$ 
- Boyve (1854-61)

- MECOS-99

- SisFrance (4. September 2009)

\section{Interpretation}

Bertrand ist der Ausgangspunkt dieser Meldung aus Neuchâtel. Da das Gebiet des heutigen Kantons Neuenburg erst 1701 zum gregorianischen Kalender wechselte, ist dieses Datum auf den 15. November vorzudatieren. Da die übrigen Kompilatoren sich auf Bertrand stützen und keine weiteren Quellen gefunden wurden, so ist auch die Umdatierung auf den 15. November höchstens als "sehr unsicher“ (wenn nicht sogar als Falschmeldung) zu werten.

\section{Bemerkungen}

Vgl. im ECOS-09 das Erdbeben vom 15. November 1593.

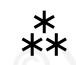
Datum
1597, 10. September
Zeit (UTC)
Ort
Simplon, vs

\section{Bewertung des Ereignisses}

Kein Erdbeben: Bergsturz.

\section{Korrektur}

Bergsturz

Überlieferung u. a. in:

- Scheuchzer (1716)

- Bertrand (1756)

- Volger (1857)

- Eisbacher (1984)

- MECOS-99

\section{Interpretation}

In den Erdbeben-Kompilationen wird das Ereignis vom 10. Sept. (jul. Kal. 31. Aug.) 1597 nur als Bergsturz beschrieben, doch im MECOS-99 wird inm irrtümlicherweise eine Intensität 6 zugeordnet. 


\begin{tabular}{|l|l|l}
\hline Datum & Zeit (UTC) & Ort \\
\hline 1599, 23. Oktober & - & Reichenau, GR
\end{tabular}

\section{Bewertung des Ereignisses}

Kein Erdbeben: Datierungsfehler.

\section{Korrektur}

1599, 2. November

\section{Überlieferung}

Candreia (1905)

\section{Interpretation}

Dieses Ereignis ist nur vom zeitgenössischen reformierten Chronisten, Maler, Lehrer und Dichter Hans Ardüser (1557-1617) in seiner Rhätischen Chronik (1877) überliefert worden. Somit ist es gregorianisch auf den 2. November 1599 umzudatieren und als ungesichert zu werten. Zitiert nach Candreia (1905, S. 35): „Den 23. October (1599) [2. Nov. greg.] was ein groser erdbidem, das Schloss Rezüns hat zittret, knelt und sich dermasen erschütt, als welt es gar zhuffen fallen. Ardüser 1 c. 152.“ Die maximale Intensität für das Ereignis am 2. November 1599 ist mit „4“ zu werten.

\section{Bemerkungen}

Vgl. im ECOS-09 den Eintrag zum 2. November 1599.

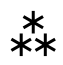

\section{Datum}

- 1600, 8. September

- 1601, 8. September
Zeit (UTC)

- 1 Uhr

\section{Ort}

- Genève

- Unterwalden

\section{Bewertung des Ereignisses}

Keine Erdbeben: Datierungsfehler

\section{Korrektur}

1601, 18. September (greg. Kal.)

\section{Überlieferung u. a. in:}

- $\quad \operatorname{Spon}(1680)$

- Bertrand (1756)

- $\quad$ Volger (1857) 


\section{Interpretation}

Wichtige Interpretations-, Abschreib- oder Druckfehler zum Unterwaldner SeptemberBeben von 1601 stammen aus der Chronik von Spon (1680), wo mit „1600“ datiert wird, aus dem gleichzeitigen Gebrauch zweier Kalenderstile mit einer Differenz von 10 Tagen und aus der Tatsache, dass das Beben kurz nach Mitternacht stattgefunden hatte. Inhaltlich scheint Spon das Beben von 1601 auch mit dem Erdbeben vom 11. März 1584 zu verwechseln.

\section{Bemerkungen}

Vgl. die Erdbeben vom 18. September 1601 in Unterwalden und das Erdbeben vom 11. März 1584 in Aigle.

\begin{tabular}{|c|c|c|}
\hline $\begin{array}{l}\text { Datum } \\
\text { 1601, 18. September }\end{array}$ & $\begin{array}{l}\text { Zeit (UTC) } \\
01 \text { Uhr }\end{array}$ & $\begin{array}{l}\text { Ort } \\
\text { Unterwalden }\end{array}$ \\
\hline
\end{tabular}

\section{Bewertung des Ereignisses}

Sicheres Ereignis.

\section{Parameter nach ECOS}

Ix: 8

\section{Publiziert in:}

- Schwarz-Zanetti G., Fäh D., Schibler R., Masciadri V., Kästli P. and Giardini D. (2003): The earthquake in Unterwalden and the rockslide from the Bürgenstock into Lake Lucerne on September 18, 1601. Eclogae geol. Helv. - Swiss J. Geosciences, 96.

- Schwarz-Zanetti, G., Deichmann, N., Fäh, D., Masciadri, V., Kästli, P., Schnellmann, M. und Jimenez, M. (2006): Das Unterwaldner Erdbeben vom 18. September 1601. Naturkatastrophen in der Zentralschweiz. Der Geschichtsfreund, 159, S. 11-28.

- Schwarz-Zanetti, G. (2008f): Das Erdbeben in Unterwalden vom 16. September 1601 und die nachfolgende Flutwelle im Vierwaldstättersee. Hsg. Gisler et al.: Nachbeben. Eine Geschichte der Erdbeben in der Schweiz, Bern.

\section{Zusammenfassung}

Am frühen Morgen des 18. September 1601 wurde Unterwalden heftig erschüttert, wobei gravierende Schäden entstanden. Etwas leichtere Schäden sind für die Stadt Luzern nachweisbar, dort war vor allem die sogenannte „Mindere Stadt“ betroffen. Neben einem Bergsturz vom Bürgenstock löste das Beben an mehreren Orten im Vierwaldstättersee auch subaquatische Rutschungen aus, deren Flutwellen die Uferpartien und nahegelegene Gebäude beschädigten und vermutlich einige Todesopfer forderten. Das Erdbeben wurde in weiten Teilen Mitteleuropas und bis nach Norditalien verspürt. 


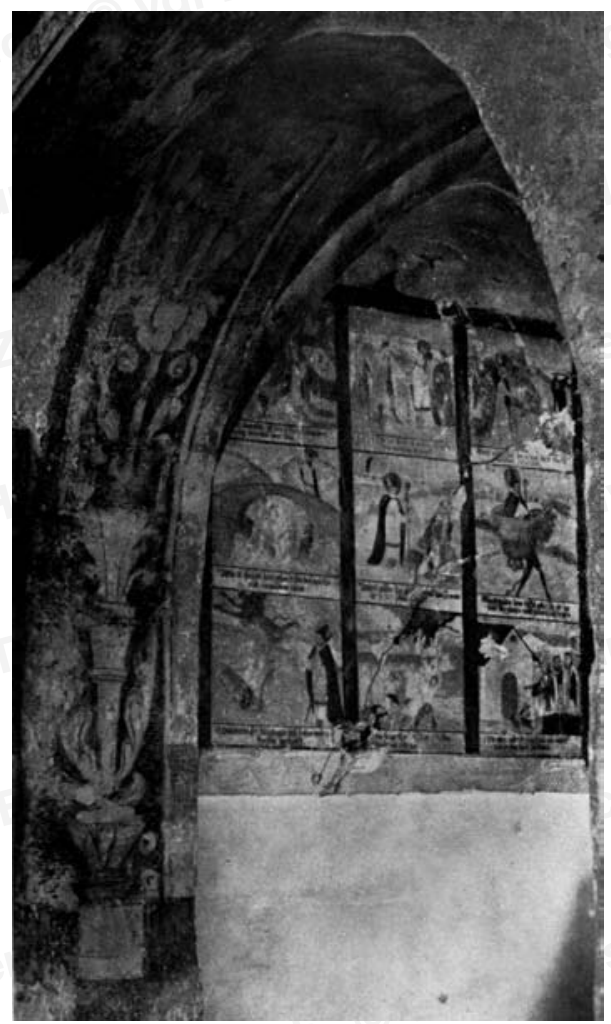

Abbildung 7: Wandbilder aus der Zeit um 1600 in der Kapelle St. Joder, Altzellen, OW, die durch eine Kopie auf Tafeln von 1620 abgedeckt waren. Für diese Kapelle sind bedeutende Schäden durch das Erdbeben vom 18. September 1601 dokumentarisch gut belegt. Sodass die deutlich sichtbaren diagonalen Risse, wie sie bei Erdbeben entstehen können, durchaus 1601 entstanden sein können. Das Bild wurde anfangs des 20. Jahrhunderts fotografiert. (Durrer, 1899-1928, S. 1090, Foto: ZB Zürich.)

Weitere Informationen zum Erdbeben 1601 sind in Weller (1862, S. 265) unter dem Titel „Busslied“ und in einer von Hans Schrötter in Basel gedruckten „Zwo wahrhafftige newe Zeitung“ (vgl. Zeitung) zu finden. 


\section{Makroseismische Parameter}

\begin{tabular}{|c|c|c|c|c|c|c|}
\hline Ortschaft & Länge & Breite & $I_{\min }$ & $I_{\max }$ & $\mathrm{I}_{\mathrm{w}}$ & Datenqualität \\
\hline Markgroeningen & 48.92 & 9.07 & & & verspürt & sehr schwach \\
\hline Heidelberg & 49.4 & 8.69 & & & verspürt & sehr schwach \\
\hline Kanton Schwyz & 47.06 & 8.69 & & & verspürt & sehr schwach \\
\hline Fegersheim & 48.48 & 7.68 & & & verspürt & sehr schwach \\
\hline Esslingen & 48.75 & 9.3 & & & verspürt & sehr schwach \\
\hline Luzern & 47.05 & 8.31 & 4 & & verspürt & sehr schwach \\
\hline Buochs & 46.97 & 8.42 & & & verspürt & sehr schwach \\
\hline Freiburg & 48 & 7.87 & & & verspürt & sehr schwach \\
\hline Talloires & 45.84 & 6.22 & & & verspürt & sehr schwach \\
\hline Obbürgen & 46.98 & 8.37 & & & verspürt & sehr schwach \\
\hline Flueelen & 46.91 & 8.63 & & 6 & verspürt & sehr schwach \\
\hline Eglisau & 47.58 & 8.52 & & & verspürt & sehr schwach \\
\hline Einsiedeln & 47.13 & 8.74 & & & verspürt & sehr schwach \\
\hline Kanton Nidwalden & 46.9 & 8.4 & 7 & 8 & 8 & schwach \\
\hline Stans & 46.96 & 8.37 & 6 & 7 & 7 & schwach \\
\hline Schaffhausen & 47.7 & 8.64 & 6 & 7 & 7 & gut \\
\hline Luzern & 47.05 & 8.29 & 7 & 8 & 7 & gut \\
\hline Zürich & 47.37 & 8.54 & 6 & 7 & 7 & mässig \\
\hline Schwyz & 47.02 & 8.65 & 6 & 7 & 7 & sehr schwach \\
\hline Oberrickenbach & 46.89 & 8.42 & 6 & 8 & 7 & schwach \\
\hline Zug & 47.17 & 8.52 & 6 & 7 & 7 & sehr schwach \\
\hline Oberwil bei Zug & 47.15 & 8.51 & 6 & 7 & 7 & sehr schwach \\
\hline Seedorf & 46.88 & 8.59 & 6 & 7 & 7 & sehr schwach \\
\hline Altzellen, OW & 46.88 & 8.38 & 6 & 7 & 7 & schwach \\
\hline Beckenried & 46.97 & 8.47 & 6 & 8 & 7 & sehr schwach \\
\hline Sisikon & 46.95 & 8.62 & 6 & 7 & 6 & sehr schwach \\
\hline Offenburg & 48.49 & 7.94 & 6 & 7 & 6 & sehr schwach \\
\hline Neuchâtel & 46.99 & 6.93 & 5 & 7 & 6 & sehr schwach \\
\hline Basel & 47.56 & 7.59 & 6 & 7 & 6 & schwach \\
\hline Maienfeld & 47.01 & 9.53 & 6 & 7 & 6 & schwach \\
\hline
\end{tabular}




\begin{tabular}{|c|c|c|c|c|c|c|}
\hline Ortschaft & Länge & Breite & $I_{\min }$ & $I \max$ & Iw & Datenqualität \\
\hline Flüeli-Ranft & 46.87 & 8.27 & 5 & 7 & 6 & schwach \\
\hline St. Gallen & 47.43 & 9.4 & 6 & 7 & 6 & schwach \\
\hline Brunnen & 47 & 8.61 & 6 & 7 & 6 & sehr schwach \\
\hline Solothurn & 47.21 & 7.54 & 6 & 7 & 6 & sehr schwach \\
\hline Chur & 46.86 & 9.53 & 5 & 6 & 5 & sehr schwach \\
\hline Eckwersheim & 48.69 & 7.7 & 4 & 6 & 5 & sehr schwach \\
\hline Lausanne & 46.52 & 6.63 & 4 & 6 & 5 & sehr schwach \\
\hline Frankfurt am Main & 50.11 & 8.65 & 4 & 6 & 5 & sehr schwach \\
\hline Kilchberg ZH & 47.32 & 8.54 & 4 & 6 & 5 & sehr schwach \\
\hline Unterstammheim & 47.64 & 8.79 & 5 & 6 & 5 & sehr schwach \\
\hline Dinhard & 47.56 & 8.77 & 5 & 6 & 5 & sehr schwach \\
\hline Stein am Rhein & 47.66 & 8.86 & 5 & 6 & 5 & sehr schwach \\
\hline Vevey & 46.46 & 6.84 & 5 & 6 & 5 & sehr schwach \\
\hline Como, I & 45.81 & 9.08 & 5 & 6 & 5 & sehr schwach \\
\hline Heiligenstein & 48.43 & 7.45 & 5 & 6 & 5 & sehr schwach \\
\hline Kanton Glarus & 46.98 & 9.06 & 4 & 6 & 5 & sehr schwach \\
\hline Colmar & 48.09 & 7.37 & 4 & 5 & 5 & sehr schwach \\
\hline Appenzell & 47.33 & 9.41 & 5 & 6 & 5 & sehr schwach \\
\hline Rümlang & 47.45 & 8.53 & 5 & 6 & 5 & sehr schwach \\
\hline Bern & 46.98 & 7.46 & 4 & 6 & 5 & sehr schwach \\
\hline Trimmis & 46.9 & 9.56 & 4 & 6 & 5 & sehr schwach \\
\hline Genève & 46.21 & 6.14 & 4 & 5 & 5 & sehr schwach \\
\hline Biel/Bienne & 47.14 & 7.25 & 5 & 6 & 5 & sehr schwach \\
\hline Frauenfeld & 47.56 & 8.89 & 5 & 6 & 5 & sehr schwach \\
\hline Sumvitg & 46.73 & 8.94 & 5 & 6 & 5 & sehr schwach \\
\hline Arth & 47.06 & 8.52 & 5 & 6 & 5 & sehr schwach \\
\hline Reichenbach & 49.72 & 8.71 & 4 & 5 & 4 & sehr schwach \\
\hline Egringen & 47.67 & 7.6 & 4 & 5 & 4 & sehr schwach \\
\hline
\end{tabular}




\section{Datum Zeit (UTC) Ort \\ \begin{tabular}{ll|l} 
1602, 28. Juni & 5 Uhr & Zürich
\end{tabular}}

\section{Bewertung des Ereignisses}

Kein Erdbeben: Datierungsfehler.

\section{Korrektur}

1602, 8. Juli, zwischen 5 und 6 Uhr morgens (4 und 5 Uhr UTC).

Überlieferung u. a. in:

- Lindiner (1711)

- Keferstein (1826)

Zeitgenössische Quelle:

- Cysat (1969)

\section{Interpretation}

In der Zürcher Kompilation von Lindiner (1711) findet man einen Hinweis auf ein Erdbeben ohne Schaden am 26. Juni 1602, jul. Datierung, ähnlich in Keferstein (1826) für Zürich am 28. Juni. Letzteren bestätigt der Zeitgenosse Cysat (1969, S. 888) in Luzern mit der gregorianischen Datierung 8. Juli. Es werden keine Schäden erwähnt.

\section{Bemerkungen}

Vgl. im ECOS-09 das Erdbeben vom 8. Juli 1602, Unterwalden.

\section{**}

Datum

1605,20 . November
Zeit (UTC)

Um 3 Uhr
Ort

Landquart, GR

\section{Bewertung des Ereignisses}

Kein Erdbeben: Datierungsfehler.

\section{Korrektur}

1605, 30. Oktober (oder 30. November)

\section{Überlieferung u. a. in:}

- Candreia (1905)

- MECOS-99

Nicht vorhanden in:

- Volger (1857) 
Zeitgenössische Quelle:

Sprecher (1992): Aus Bartholomäus Anhorns Chronik der Stadt Maienfeld.

\section{Interpretation}

Sprecher (1992, S. 349) führt in seiner Edition von Anhorn den 20. Oktober auf, der als julianisches Datum auf den 30. Oktober vordatiert werden muss: „1605. Den 20. Octobris am morgen um vier uren habend wir gar ein starcken erdbidem gespeürt." Doch Candreia (1905, S. 38) schrieb dieses Erdbeben ohne Belege dem 20. November 1605 zu, da Anhorn das Datum verwechselt habe. Solange kein weiteres Datenmaterial zur Verfügung steht, kann Candreias Argumentation nicht gewertet werden.

\section{Bemerkungen}

Vgl. im ECOS-09 das Erdbeben vom 30. Oktober 1605.

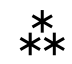

\begin{tabular}{l|l|l} 
Datum & Zeit & Ort \\
$1610,29$. November & - & Basel
\end{tabular}

\section{Bewertung des Ereignisses}

Kein Erdbeben: Datierungsfehler.

\section{Korrektur}

1610, 9. Dezember

Überlieferung u. a. in:

- Gross (1624)

- Bertrand (1756)

- Volger (1857)

- Montandon (1942-43)

- MECOS-99

\section{Interpretation}

Gross (1624) lebte im reformierten Basel, wo zu jenem Zeitpunkt noch im julianischen Stil datiert wurde, sodass das Datum um 10 Tage vorverschoben werden muss.

\section{Bemerkungen}

Vgl. das Erdbeben vom 9. Dezember 1610. 


\section{Datum \\ 1610, 9. Dezember

\section{Bewertung des Ereignisses}

Sehr unsicher.

\section{Parameter nach ECOS}

Ix: 7

\section{Überlieferung u. a. in:}

- Rahn (1690)

- Bertrand (1756)

- Hoff (1840-1841)

- Perrey (1845)

- $\quad$ Langenbeck (1892)

Nicht vorhanden in:

SisFrance (1. September 2009)

\section{Quelle}

Die einzige bekannte zeitgenössische Beobachtung stammt von Gross (1624). Keine Hinweise bei weiteren zeitgenössischen Beobachtern, wie zum Beispiel bei den Bündnern Hans Ardüser und Bartholomäus Anhorn (hsg. von Sprecher, 1992) oder beim Luzerner Renward Cysat.

\section{Interpretation}

Der Hinweis in der Chronik von Gross ist der einzige, der dieses Erdbeben und den Schaden beschreibt. Gross (1624, S. 238): 1610, Erdbidem (Marginalnotiz): „Den 29. Novembris (9. Dez., greg. Kal.) war ein Erdbidem/gab ein gross gethöss von sich. Damaln fiel ein stuck an der ausseren Ringmauren eyn bey dem Spalen-Thor. Item ein Thurn im jnneren Stattgraben/darüber man sich zuvor bemühet/wie er alss bawloss mit geringem kosten abzubrechen were.“

Diese doch relativ späte Einzel-Beschreibung und der möglicherweise schlechte bauliche Zustand des Turms lassen nur eine Wertung „sehr unsicher“ zu.

Makroseismische Parameter

\begin{tabular}{|l|l|l|l|l|l|l|}
\hline Ortschaft & Länge & Breite & $I_{\min }$ & $I_{\max }$ & $I_{w}$ & Datenqualität \\
\hline Basel & 47.56 & 7.59 & 6 & 7 & 7 & sehr schwach \\
\hline
\end{tabular}




\begin{tabular}{l|l|l} 
Datum & Zeit & Ort \\
$1612,9$. November & - & Neuchâtel
\end{tabular}

\section{Bewertung des Ereignisses}

Kein Erdbeben: Datierungsfehler.

\section{Korrektur}

Eventuell 19. November 1612

\section{Überlieferung u. a. in:}

- Boyve (1854-1855, S. 131)

- $\quad$ Kopp (1861)

- Tribolet (1876)

- MECOS-99

\section{Interpretation}

Die Kompilatoren Kopp (1861) und Tribolet (1876) vermerken in ihren Listen ohne Quellenverweise ein Erdbeben am 9. November 1612 in Neuenburg, letzterer stützt sich teilweise auch auf Boyve (1854-55). Da der Kanton Neuenburg erst 1701 zum gregorianischen Kalender überging, dürfte es sich bei diesem Datum um den 19. November 1612 handeln. Weil jedoch keine zeitgenössischen Quellen nachweisbar sind, muss der Hinweis insgesamt als „sehr unsicher“ gewertet werden. Möglicherweise handelt es sich sogar um eine Verwechslung mit dem Basler Beben vom 29. (19.) November 1610.

\section{Bemerkungen}

Vgl. im ECOS-99 das Erdbeben vom 19. November 1612.

\section{***}

\begin{tabular}{l|l|l} 
Datum & Zeit & Ort \\
$-1614,17$. Feb. & - & - Basel \\
$-1614,28$. Feb. & - & - Basel
\end{tabular}

\section{Bewertung des Ereignisses}

Kein Erdbeben: Datierungsfehler

\section{Korrektur}

1614, 27. Februar 
Überlieferung u. a. in:

- Montandon (1942-43)

- MECOS-99

\section{Quellen}

- Klauber (1617)

- Gross (1614 und 1624)

\section{Interpretation}

Gross (1614), und damit auch sein Kopist Johann Rudolf Klauber in seiner "Nachtigall“ genannten Beschreibung der Stadt Basel aus dem Jahr 1617, datieren das Erdbeben auf den 17. Februar 1614.

Da aber der Kanton Basel-Stadt erst 1701 zum gregorianischen Stil überging, muss es sich um eine julianische Datierung handeln, bzw. um den 27. Februar 1614 im heutigen Stil. Montandon (1942-43) hingegen datiert das Ereignis irrtümlicherweise auf den 18./28. Februar und nennt es, ohne Quellen angeben zu können „l'un des plus violents séismes qui aient été ressentis à Bâle; on l'a signalé aussi à Dorneck (SO)“. In den Quellen werden jedoch nirgends Schäden erwähnt.

\section{Bemerkungen}

Vgl. im ECOS-09 das Erdbeben vom 27. Februar 1614.

\begin{tabular}{|c|c|c|}
\hline $\begin{array}{l}\text { Datum } \\
\text { 1614, 24. September }\end{array}$ & $\begin{array}{l}\text { Zeit (UTC) } \\
0 \text { Uhr } 15\end{array}$ & $\begin{array}{l}\text { Ort } \\
\text { Aesch - Basel }\end{array}$ \\
\hline
\end{tabular}

\section{Bewertung des Ereignisses}

Kein Erdbeben: Datierungsfehler.

\section{Korrektur}

1614, 4. Oktober

\section{Überlieferung u. a. in:}

- Haffner (1666)

- Scheuchzer (1716)

- Bertrand (1756)

- Volger (1857)

- Sieberg (1940)

- Montandon (1942-43) 
Zeitgenössische Quellen:

- StASaR: Kirchenbücher Stein am Rhein: Ki 1.

- Schaltegger (1913)

- Klauber (1617)

- Gross (1614 und 1624)

\section{Interpretation}

Aus den zeitgenössischen Kirchenbüchern von Stein am Rhein, der Kappeler Chronik von Frauenfeld (Schaltegger 1913), den Chroniken von Gross (1614 und 1624) und Klauber (1617) lässt sich zuverlässig auf ein Erdbeben ohne Schaden im Herbst 1614 schliessen. Da aber die Kantone Schaffhausen und Basel sowie die Gemeine Herrschaft Thurgau bis 1701 den julianischen Kalender verwendeten, muss das Ereignis auf den heutigen gregorianischen Kalender, also auf den 4. Oktober 1614, umdatiert werden.

\section{Bemerkungen}

Vgl. im ECOS-09 das Erdbeben vom 4. Oktober 1614 in Basel.

\section{$\stackrel{*}{* *}$}

\begin{tabular}{l|l|l} 
Datum & Zeit (UTC) & Ort \\
$-1616,19$. Februar & -4 Uhr 30 & - Zürich \\
$-\quad 1616,20$. März & - & - Engelberg, OW
\end{tabular}

\section{Bewertung des Ereignisses}

Keine Erdbeben: Datierungsfehler.

\section{Korrektur}

1616, 29. Februar

\section{Überlieferung u. a. in:}

- Perrey (ohne Jahr), zitiert in Volger (1857)

- Volger (1857)

- Sieberg (1940)

- Gils, Van \& Leydecker (1991)

- Ortelli \& Albini (1998)

- DOM4.1

- MECOS-99

\section{Quellen}

- StAZH: E III 062.1: Pfarrbuch aus Kilchberg, ZH.

- Candreia (1905) zitiert Bartholomäus Anhorn, Pfarrer in Maienfeld, der eine Beobachtung in Zürich festhielt. 


\section{Interpretation}

Zum einen handelt es sich um einen Kalenderfehler. Denn es lässt sich ein Erdbeben am 29. Februar 1616, greg. Datierung, morgens zwischen 5 und 6 Uhr Ortszeit anhand des Pfarrbuchs aus Kilchberg, ZH (StAZH: E III 062.1) und von Bartholomäus Anhorn (zitiert in Candreia 1905) für Zürich zeitgenössisch nachweisen, doch erwähnen beide keine Schäden, weshalb dieses Erdbeben am korrigierten Datum mit der Intensität 5 bewertet wurde.

Zum andern aber spricht Volger (1857) von einem Schadenbeben in der Schweiz anfangs März 1616. Er beruft sich dabei auf Perrey, der den zeitgenössischen französischen Historiker Claude Malingre dit St. Lazare zitiert: Remarques d'Estat et d'histoire de 1600 à 1632, p. 125, was hier nicht überprüft werden konnte. Kein Hinweis in SisFrance. Die Herkunft der Lokalisierung Engelberg in der Kompilation Van Gils \& Leydeckers und in der Arbeit von Ortelli und Albini konnte nicht eruiert werden. Darum muss das Märzdatum vorläufig als Falschmeldung gewertet werden.

\section{Bemerkungen}

Vgl. im ECOS-09 das Erdbeben vom 29. Februar 1616.

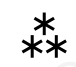

\begin{tabular}{|l|l|l} 
Datum & Zeit (UTC) & Ort \\
\hline 1616, 29. Februar & 04 Uhr & Nicht lokalisierbar (Zürich)
\end{tabular}

\section{Bewertung des Ereignisses}

Sicheres Ereignis.

\section{Parameter nach ECOS}

Ix: 5

\section{Überlieferung}

- Perrey (ohne Jahr), zitiert in Volger (1857)

- $\quad$ olger (1857)

- $\quad$ Sieberg (1940)

- Gils, Van \& Leydecker (1991)

- Ortelli \& Alibini (1998)

- DOM4.1

\section{Quellen}

- StAZH: E III 062.1: Pfarrbuch aus Kilchberg, ZH.

- Candreia (1905) zitiert Bartholomäus Anhorn, Pfarrer in Maienfeld, der eine Beobachtung in Zürich festhielt. 


\section{Interpretation}

Die Quellen und Kompilationen enthalten mit der Datierung „19. Februar“ eine julianische Datierung. Doch es lässt sich ein Erdbeben am 29. Februar 1616, greg. Datierung, morgens zwischen 5 und 6 Uhr Ortszeit anhand des Pfarrbuchs aus Kilchberg, Kanton ZH (StAZH: E III 062.1) und von Bartholomäus Anhorn (zitiert in Candreia 1905) für Zürich zeitgenössisch nachweisen, beide erwähnen keine Schäden.

Zum andern aber spricht Volger (1857) von einem Schadenbeben in der Schweiz anfangs März 1616. Er beruft sich dabei auf Perrey, der den zeitgenössischen französischen Historiker Claude Malingre, dit St. Lazare, zitiert: Remarques d'Estat et d'histoire de 1600 à 1632, p. 125, was hier nicht überprüft werden konnte. Kein Hinweis in SisFrance. Die Herkunft der Lokalisierung Engelberg in der Kompilation Van Gils \& Leydeckers und in der Arbeit von Ortelli und Albini konnte nicht eruiert werden. Weil auch anzunehmen ist, dass ein Schadenbeben im 17. Jahrhundert deutliche Spuren in den Chroniken hinterlassen hätte, ist hier vorläufig mit „sehr unsicher“ und mit $I_{w}=5$ zu werten. Trotzdem ist dieses Datum bei künftigen Quellensuchen im Auge zu behalten.

\section{Makroseismische Parameter}

\begin{tabular}{|l|l|l|l|l|l|l|}
\hline Ortschaft & Länge & Breite & $I_{\min }$ & $I_{\max }$ & $I_{\text {w }}$ & Datenqualität \\
\hline Kilchberg, ZH & 47.32 & 8.54 & & & verspürt & sehr schwach \\
\hline Zürich & 47.37 & 8.54 & 4 & 6 & 5 & sehr schwach \\
\hline
\end{tabular}

\begin{tabular}{l|l|l} 
Datum & Zeit (UTC) & Ort \\
$-\quad 1618,25$. August & $-\quad$ anfangs der & $-\quad$ Chiavenna, I \\
$-\quad 1618,4$. September & Nacht & - Plurs/Piuro, I
\end{tabular}

\section{Bewertung des Ereignisses}

Kein Erdbeben, sondern Bergsturz am 4. September in Plurs/Piuro, I.

\section{Publiziert in:}

- Scaramellini, G., Kahl, G., Falappi G. (1988): La frana di Piuro del 1618. Storia e immagini di una rovina (Grundlagenwerk).

- Schwarz-Zanetti, Gabriela (2008h): Der Bergsturz von Plurs vom 4. September 1618. Exkurs. Nachbeben. Eine Geschichte der Erdbeben in der Schweiz, Hsg. Gisler et al., Bern, S. 74-75.

\section{Bemerkung}

Der Bergsturz von Plurs am 4. September 1618 (greg. Datierung) fand im Anschluss an eine Regenperiode statt und forderte mindestens 900 Todesopfer, vgl. auch die Arbeit von Poli \& Ghilardi (1988). Die Niederschlagsverhältnisse sollten noch genauer unter- 
sucht werden, denn die Monate August und September 1618 weisen nicht nur im Bergell starke Regenfälle auf, sondern gemäss Climhist von C. Pfister (1984 und 2009) auch in weiteren Teilen der Schweiz, was zu Überschwemmungen und im Tessin zu einem weiteren Erdrutsch führte.

Zusätzlich lässt sich für Belmont bei Pully, VD, dank der Aufzeichnungen des Pfarrers François Dind (Dumur 1907, S. 330), ein beinah gleichzeitiger, verheerender Erdrutsch nachweisen: „Mémoire à la postérité que la 24e d'aoust 1618 [3.9. greg.], jour de la St-Barthélemi, est arrivé, environ 11 heures devant midi, un grand dégast et désasstre ès vignes de Bemont, du costé d'orient. La maison de la Conversion s'est aussi glissée et les vignes et possessions et arbres sont renversez et remuez. Plusieurs voisins y ont estés et moi avec eux pour recercher le mesnage de la maison engouffrée en terre. Dieu ait merci de nous."

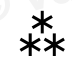

\begin{tabular}{|c|c|c|}
\hline $\begin{array}{l}\text { Datum } \\
-\quad(1621,20 . \text { Mai) } \\
-\quad 1621,21 . \text { Mai }\end{array}$ & $\begin{array}{l}\text { Zeit (UTC) } \\
- \\
-\end{array}$ & $\begin{array}{l}\text { Ort } \\
-\quad \text { Neuchâtel } \\
-\quad \text { Neuchâtel }\end{array}$ \\
\hline
\end{tabular}

\section{Bewertung des Ereignisses}

Keine Erdbeben: Datierungsfehler.

\section{Korrektur}

1621, 30. und 31. Mai

\section{Überlieferung u. a. in:}

- Spon (1680)

- Bertrand (1756)

- Guéneau de Montbelliard (1761)

- Volger (1857)

- Langenbeck (1892)

- Sieberg (1940)

Zeitgenössischer Bericht aus Neuenburg mit jul. Datierung:

StANE Sign.: Livres de raison/Journaux LRJ 22. Favarger: Journal 1547-1681 (avec lacunes): „Le 20 May 1621 par un Jour de Pentecoste Dieu first trembler la terre par trois fois et le lendemain elle trembla encor .... ." (p. 148)

Zeitgenössische Berichte mit greg. Datierung oder mit dem Begriff „Pfingsten“:

- Gross (1624): Basel Kurtze Bassler Chronick

- FAHASM: DIV/13/0/1. Chronique du chanoine Bérodi. 
- Dumur (1907, S. 329): Notes extraites des registres de l'état-civil de la paroisse de Pully.

- $\quad$ ZHBL, ohne Signatur: Das Familienarchiv Amrhyn. Akten, Brülisauer (1982, S. 407)

\section{Interpretation}

Die aufgeführten Kompilationen und zeitgenössischen Notizen, ausser Amrhyn (ZHBL), haben die julianische Datierung verwendet, darum die Korrektur auf den 30. und 31. Mai 1621.

\section{Bemerkungen}

Vgl. die Erdbeben vom 30. und 31. Mai 1621.

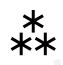

\section{Datum}

- 1621, 30. Mai

- 1621, 31. Mai
Zeit (UTC)

- (13 Uhr)
Ort

- Neuchâtel

- Neuchâtel

\section{Bewertung des Ereignisses}

Sichere Ereignisse.

\section{Parameter nach ECOS}

- Ix:30. Mai: 5

- 31. Mai: verspürt

\section{Überlieferung u. a. in:}

- Spon (1680)

- Bertrand (1756)

- Volger (1857)

- Montandon (1942-43)

- MECOS-99

\section{Quellen}

- StANE, Sign.: Livres de raison/Journaux LRJ 22. Favarger: Journal 1547-1681.

- Gross (1624): Basel Kurtze Bassler Chronick.

- FAHASM: DIV/13/0/1. Chronique du chanoine Bérodi.

- Dumur (1907, S. 329): Notes extraites des registres de l'état-civil de la paroisse de Pully.

- ZHBL, ohne Signatur: Das Familienarchiv Amrhyn. Akten, Brülisauer (1982, S. 407).

\section{Interpretation}

Jonas Favarger, Pfarrer an einem ungenannten Ort im Kanton Neuenburg, hält in seinem Tagebuch für Pfingsten, den 30. Mai 1621, drei Erdbeben und für den 31. Mai ein viertes fest. Er ist der einzige Beobachter, der von insgesamt vier Beben an zwei aufeinan- 
derfolgenden Tagen spricht: „Le 20 May 1621 par un Jour de Pentecoste Dieu fist trembler la terre par trois fois et le lendemain elle trembla encor Dieu nous fasse trembler a la denonciacion de ses justes jugemens." (p. 148)

Das Ereignis ist mit vier zeitgenössischen Quellen zwar gesichert, doch die zeitliche Abfolge der Serie aus Favarger kann noch nicht rekonstruiert werden. Eine Notiz aus dem Zivilstandsregister aus Pully bei Lausanne, VD, von Pfarrer Dind fixiert ein Erdbeben auf zwei Uhr nachmittags. In St. Maurice, VS, notiert Bérodi drei Uhr nachmittags für seine Beobachtung. In Luzern (Familienarchiv Amrhyn) und Genf (Spon, 1680), wovon nur Abschriften ungenannter Quellen bekannt sind, wird das Beben während zeitlich nicht näher beschriebenen Messfeiern verspürt, in Basel handelt es sich um die Abendpredigt. Die Intensität der Beben wird in den Quellen aus Neuenburg und Pully überhaupt nicht beschrieben, während sich die Beobachter in Luzern und Genf scheinbar gefürchtet haben. In St. Maurice charakterisiert Bérodi das Beben mit „stark“. Schäden werden aber in diesen vier zeitgenössischen Quellen nirgends erwähnt. Ein anderes Erklärungsmodell würde die Annahme von mehreren Beben an verschiedenen Orten zu nur leicht unterschiedlichen Zeitpunkten bieten.

Erst Bertrand (1756) und seine Kopisten schreiben von herabgefallenen Kaminen in Neuenburg, ohne einen Quellenbeleg zu geben, sodass momentan von einer Erdbebenserie unter der Schadensgrenze ausgegangen werden muss.

\section{Makroseismische Parameter}

30. Mai 1621:

\begin{tabular}{|l|l|l|l|l|l|l|}
\hline Ortschaft & Länge & Breite & $I_{\min }$ & $I_{\max }$ & $I_{\text {w }}$ & Datenqualität \\
\hline Basel & 47.56 & 7.59 & & & verspürt & sehr schwach \\
\hline Pully & 46.52 & 6.66 & & & verspürt & sehr schwach \\
\hline Biel/Bienne & 47.14 & 7.25 & 4 & 6 & 5 & sehr schwach \\
\hline Fribourg & 46.81 & 7.16 & 4 & 5 & 5 & sehr schwach \\
\hline Genève & 46.21 & 6.14 & 4 & 5 & 5 & schwach \\
\hline Luzern & 47.05 & 8.29 & 4 & 5 & 5 & sehr schwach \\
\hline St-Maurice & 46.22 & 7 & 4 & 5 & 5 & sehr schwach \\
\hline Bern & 46.98 & 7.46 & 4 & 5 & 5 & sehr schwach \\
\hline Neuchâtel & 46.99 & 6.93 & & & 5 & sehr schwach \\
\hline
\end{tabular}

31. Mai 1621:

\begin{tabular}{|l|l|l|l|l|l|l|}
\hline Ortschaft & Länge & Breite & $I_{\min }$ & $I_{\max }$ & $I_{w}$ & Datenqualität \\
\hline Neuchâtel & 46.99 & 6.93 & & & verspürt & sehr schwach \\
\hline
\end{tabular}




\section{Datum Zeit $\quad$ Ort

\begin{tabular}{|c|}
\hline 1622, 1. Mär \\
\hline
\end{tabular}

\section{Bewertung des Ereignisses}

Kein Erdbeben: Datierungsfehler.

\section{Korrektur}

1623, 1.-6. März: Erdbebenserie im Veltlin, Puschlav und Bergell, lokalisiert in Chiesa, I.

\section{Überlieferung u. a. in:}

- Scheuchzer (1706)

- Bertrand (1756)

- $\quad$ Candreia (1905)

- $\quad$ Volger (1857)

- MECOS-99

Keine Hinweise in:

- Baratta (1901) zu 1622 und 1623

- Italienischer Katalog DOM4.1

Zeitgenössische Quellen, zitiert nach Candreia (1905, S. 42-45):

- 1622, 1. März: Cellario, Msc. Kat Raet. I., 210. 142, zit C.S. (?), p. 369., für Poschiavo und Brusio

- 1623, 20.-24. Feb. (jul.)/2.-6. März (greg.), Anhorn: Graw Pündtner Krieg, Msc. Kat. Raet. 202, 51, pag. 373 und Msc. Kat Raet. 202, 52

- $\quad$ 1623, 1. März (greg.), Msc. Ziz. Samml. XIV. 238., für das Veltlin

\section{Interpretation}

Candreia (1905, S. 42-45) enthält ähnliche Tages-Datierungen für zwei Erdbebenserien in den Jahren 1622 und 1623 mit julianischen und gregorianischen Tagesangaben, ohne dass ein möglicher Zusammenhang diskutiert wird.

Die Ähnlichkeit der Datierung und der Lokalisierung ist aber derart gross, dass von einem Lese- oder Abschreibfehler ausgegangen werden muss. Da für die 1623-Serie zwei ungefähr übereinstimmende Berichte vorhanden sind, so ist der Fehler eher bei der Jahresangabe 1622 zu vermuten.

\section{Bemerkungen}

Vgl. im ECOS-09 das Erdbeben in Chiesa, I, am 2. März 1623.

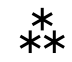




\section{Datum \\ Zeit (UTC) \\ Ort \\ 1622, 24. Juli \\ $22 \mathrm{Uhr}$ \\ Unterengadin}

\section{Bewertung des Ereignisses}

Kein Ereignis: Datierungsfehler.

\section{Korrektur}

1622, 3. August

Überlieferung u. a. in:

- Volger (1857)

- Candreia (1905)

Zeitgenössische Quellen:

- Anhorn, Bartholomäus (1873)

- Sprecher, Fortunat, zit. in Candreia (1905)

Publiziert in:

- Albini (1994)

- $\quad$ Gisler et al. (2005, S. 64-67)

\section{Interpretation}

Bei dieser Datierung handelt es sich um eine julianische, die Zusammenfassung der dazugehörigen Publikationen ist unter dem korrigierten gregorianischen Datum zu finden.

\section{Bemerkungen}

Vgl. das Erdbeben vom 3. August 1622.

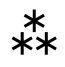

\begin{tabular}{|l|l|l}
\hline Datum & Zeit & Ort \\
\hline $1622,3$. August & - & Ftan, GR
\end{tabular}

\section{Bewertung des Ereignisses}

Sicher.

\section{Parameter nach ECOS}

Ix: 7 


\section{Publiziert in:}

- Albini, P. et al. (1994): Alcuni terremoti importanti per l'Alta Valtellina. Studi sismici in Alta Valtellina, ISMES-IRRS (CNR), n. 336. p. 25-50.

- Gisler, M., Weidmann, M., Fäh, D., Kästli, P., Schwarz-Zanetti, G., Deichmann, N. (2005): Erdbeben in Graubünden. Vergangenheit, Gegenwart, Zukunft. S. 64-67.

Zusammenfassung nach Gisler et al. (2005), S. 64-67:

Zwei zeitgenössischen Quellen ist zu entnehmen, dass am 3. August 1622 im Unterengadin zwei Erdbeben verspürt worden sind, und zwar nachts zwischen 23 und 24 Uhr und um 1 Uhr Lokalzeit, beziehungsweise zwischen 22 und 23 Uhr und um 00 Uhr UTC. In dieser Gegend, die zu jenem Zeitpunkt auch von den Wirren des Dreissigjährigen Kriegs betroffen war, seien grössere Schäden, wie zum Beispiel das Zusammenstürzen überwölbter Räume, entstanden. In Ftan habe eine zusammenstürzende Mauer sogar zwei Kinder erschlagen. Dabei ist unklar, in welchem Zustand sich diese Mauer im kriegsversehrten Ftan befunden hatte. Obwohl das Ereignis historisch gesichert ist, ist die Intensität 7 sehr unsicher.

\section{Makroseismische Parameter}

\begin{tabular}{|l|l|l|l|l|l|l|}
\hline Ortschaft & Länge & Breite & $I_{\min }$ & $I_{\max }$ & $I_{\text {w }}$ & Datenqualität \\
\hline Sent & 46.82 & 10.34 & & & verspürt & sehr schwach \\
\hline Ftan & 46.79 & 10.25 & 6 & 7 & 7 & sehr schwach \\
\hline Kanton Graubuenden & 46.61 & 9.57 & 1 & 3 & 1 & sehr schwach \\
\hline Chur & 46.86 & 9.53 & 1 & 3 & 1 & sehr schwach \\
\hline
\end{tabular}

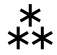

Datum

1623, 20. Februar

\section{Zeit}

$-$

\section{Ort}

Chiesa, I

\section{Bewertung des Ereignisses}

Kein Erdbeben: Datierungsfehler.

\section{Korrektur}

1623, 2. März (Erdbebenserie vom 2.-5. März, greg. Kal.)

\section{Überlieferung u. a. in:}

- Candreia (1905)

- Gils, Van \& Leydecker (1991)

- MECOS-99 


\section{Interpretation}

Die in Candreia (1905) zitierten bündnerischen Quellen datieren den Beginn der Erdbebenserie im julianischen Kalenderstil und sind entsprechend um 10 Tage nach vorne zu korrigieren.

\section{Bemerkungen}

Vgl. im ECOS-09 das Erdbeben vom 2. März 1623.

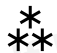

\begin{tabular}{|l|l|l} 
Datum & Zeit (UTC) & Ort \\
$-\quad 1635,11$. Juni & -23 Uhr & - Zürich \\
$-\quad 1635,21$. Juni & -23 Uhr & - Zürich
\end{tabular}

\section{Bewertung der Ereignisse}

Keine Erdbeben: Datierungsfehler.

\section{Korrektur}

1635, 20. Juni

\section{Überlieferung $u$. a. in:}

- Ziegler (1674)

- Scheuchzer (1716)

- Volger (1857)

- MECOS-99

\section{Quelle}

StAZH: E III 79.1: Pfarrbuch Niederglatt und Niederhasli

\section{Interpretation}

Die Pfarrbücher von Niederglatt und Niederhasli zeigen eindeutig, dass die Datierung einen Kalender- und einen Nachtfehler enthält, damit ist das Datum um 9 Tage zu korrigieren. Es gibt keinen Hinweis auf Schäden, weshalb das Ereignis vom 20. Juni mit einer Intensität „verspürt“ bewertet wurde.

\section{Bemerkungen}

Vgl. das Erdbeben vom 20. Juni 1635.

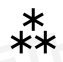




\section{Datum \\ 1635,20 . Juni \\ Zeit (UTC) \\ Ort \\ $21 \mathrm{Uhr}$ \\ Nicht lokalisierbar (Zürich)}

\section{Bewertung des Ereignisses}

Unsicher.

\section{Parameter nach ECOS}

Ix: verspürt

\section{Überlieferung u. a. in:}

- Ziegler (1674)

- Scheuchzer (1716), zitiert J.H. Hottinger (1665)

- Volger (1857)

- MECOS-99

\section{Quelle}

StAZH: E III 79.1, S. 349), Pfarrbuch Niederglatt und Niederhasli: „Den 10 lunij ist in disem jar [1635] in der nacht inter 10 und 11 hore noctu [ist] ein erdbidem gsin."

Keine Fakten vorhanden in:

Lavater (1635): Theses de terrae motu. Tiguri. Disputation vom 2. Juli 1635 (jul. Dat.; 30. Juni, greg.), 11 Seiten. Darin wird nur indirekt auf das aktuelle Ereignis Bezug genommen.

\section{Interpretation}

Das Pfarrbuch von Niederglatt darf als zeitgenössische Quelle mit julianischer Datierung gewertet werden. Bestätigt wird sie von Scheuchzer (1716), der aus dem „Speculum Helvetico Tigurinum“ (1665) des Zürchers Johann Heinrich Hottinger (1620-1667) zitiert, er datiert jedoch auf den 11. Juni, jul. Kalender und stuft es als "gering“ ein.

\section{Makroseismische Parameter}

\begin{tabular}{|l|l|l|l|l|l|l|}
\hline Ortschaft & Länge & Breite & $I_{\min }$ & $I_{\max }$ & $I_{\text {w }}$ & Datenqualität \\
\hline Niederhasli & 47.48 & 8.48 & & & verspürt & sehr schwach \\
\hline Zürich & 47.37 & 8.54 & & & verspürt & sehr schwach \\
\hline
\end{tabular}

\section{$\stackrel{*}{* *}$}

\begin{tabular}{|l|l|l|}
\hline Datum & Zeit (UTC) & Ort \\
\hline 1642, 3. Juni & 21 Uhr 30 & Piz d'Err, GR
\end{tabular}

\section{Bewertung des Ereignisses}

Kein Erdbeben: Datierungsfehler. 


\section{Korrektur}

1642, 13. Juni, Bergamo, I

\section{Überlieferung u. a. in:}

- Baratta (1901)

- Candreia (1905)

- Gils, Van \& Leydecker (1991)

- MECOS-99

- DOM4.1

Zeitgenössische Beobachtungen aus der Schweiz:

StiAEi: A. HB 101: Abt Placidus, Einsiedeln und Pfäffikon

\section{Interpretation}

Die Datierung 3. Juni 1642 in den Kompilationen ist julianisch, wie die Beschreibung im gregorianischen Diarium des Abtes Placidus für Einsiedeln, Pfäffikon, SZ, und Mailand zeigt: „1642, Juni 13. Nocte sequenti circa undecimam terremotus fuit a pluribus notatus hic, et Pfefficonensae, et alibi: maxime Mediolano“. [13. Juni 1642. In der folgenden Nacht, ungefähr um 11 Uhr (23 Uhr Ortszeit), hat es ein Erdbeben gegeben, hier (Einsiedeln, SZ) von mehreren bemerkt, und in Pfäffikon (SZ) und anderswo; am stärksten in Mailand.]

Der in Candreia (1906, S. 45) zitierte Hinweis auf Thusis aus dem Manuskript Rosenroll muss deshalb auf den 13. Juni 1642 umdatiert werden. Diese Beobachtungen gehören damit zum Schadenbeben von Bergamo vom 13. Juni 1642 (DOM4.1, lo = 6.5). Das Erdbeben ist in italienischen Katalogen dokumentiert. Die Herkunft der Lokalisierung „Piz d'Err“ konnte nicht mehr eruiert werden.

\section{Bemerkungen}

Vgl. das Erdbeben vom 13. Juni 1642.

\begin{tabular}{|c|c|c|}
\hline $\begin{array}{l}\text { Datum } \\
\text { 1642, 13. Juni }\end{array}$ & $\begin{array}{l}\text { Zeit (UTC) } \\
22 \text { Uhr }\end{array}$ & $\begin{array}{l}\text { Ort } \\
\text { Bergamo, I }\end{array}$ \\
\hline
\end{tabular}

\section{Bewertung des Ereignisses}

Sicher.

\section{Parameter nach ECOS}

Ix: 6.5

\section{Überlieferung}

- $\quad$ Baratta (1901)

- Candreia (1905) 
- Gils, Van \& Leydecker (1991)

- MECOS-99

- DOM4.1

\section{Quelle}

Abt Placidus, Einsiedeln und Pfäffikon (StiAEi: A. HB 101).

\section{Interpretation}

Das Diarium des Einsiedler Abtes Placidus enthält eine Eigenbeobachtung für Einsiedeln, aber auch Informationen aus Pfäffikon, SZ, und Mailand: 1642, Juni 13. Nocte sequenti circa undecimam terremotus fuit a pluribus notatus hic, et Pfefficonensae, et alibi: maxime Mediolano. [13. Juni: In der folgenden Nacht hat es ein Erdbeben gegeben, hier von mehreren bemerkt, und in Pfäffikon (SZ) und anderswo; am stärksten in Mailand.]

Die übrigen Schweizer Hinweise stammen aus Kopien, die nicht weiter überprüft werden konnten.

\section{Makroseismische Parameter (ohne italienische Sitepoints)}

\begin{tabular}{|l|l|l|l|l|l|l|}
\hline Ortschaft & Länge & Breite & $I_{\min }$ & $I_{\max }$ & $I_{\text {w }}$ & Datenqualität \\
\hline Thusis, GR & 46.7 & 9.43 & & & verspürt & sehr schwach \\
\hline Chur, GR & 46.86 & 9.53 & & & verspürt & sehr schwach \\
\hline Sent, GR & 46.82 & 10.34 & & & verspürt & sehr schwach \\
\hline Medels im Rheinwald, GR & 46.55 & 9.3 & 4 & 5 & 5 & sehr schwach \\
\hline Mailand, I & 45.47 & 9.19 & 4 & 6 & 5 & sehr schwach \\
\hline Pfäffikon, SZ & 47.2 & 8.78 & 4 & 5 & 4 & sehr schwach \\
\hline Einsiedeln, SZ & 47.13 & 8.74 & 4 & 5 & 4 & sehr schwach \\
\hline
\end{tabular}

**

\section{Datum}

1648, 23. November
Zeit (UTC)

$-$

\section{Ort}

Neuchâtel

\section{Bewertung des Ereignisses}

Kein Erdbeben: Datierungsfehler.

\section{Korrektur}

1648, 3. Dezember

\section{Überlieferung u. a. in:}

- Bertrand (1756)

- Boyve (1854-1858)

- Volger (1857) 
- MECOS-99

- SisFrance

\section{Interpretation}

Da die Kantone Neuenburg und Waadt den Kalenderstil erst 1701 wechselten, ist die Datierung mit grosser Wahrscheinlichkeit auf den 3. Dezember zu korrigieren. Dieses Beben ohne Schäden wiederum kann nur als sehr unsicher gewertet werden, da sich der älteste Nachweis in der Kompilation von Bertrand aus dem Jahre 1756 befindet: „Le ving-troisième de Novembre, mille six-cent quarante-huit, on apperçut quelques secousses dans le Comté de Neufchâtel. (...) On ressentit les mêmes secousses à Yverdon.“

\section{Bemerkungen}

Vgl. das Erdbeben vom 3. Dezember 1648.

\begin{tabular}{|c|c|c|}
\hline $\begin{array}{l}\text { Datum } \\
\text { 1648, 3. Dezember }\end{array}$ & Zeit & $\begin{array}{l}\text { Ort } \\
\text { Nicht lokalisierbar (Neuchâtel) }\end{array}$ \\
\hline
\end{tabular}

\section{Bewertung des Ereignisses}

Sehr unsicheres Ereignis.

\section{Parameter nach ECOS}

Ix: verspürt

\section{Überlieferung}

- $\quad$ Bertrand (1756)

- Boyve (1858)

- Volger (1857)

- MECOS-99

- SisFrance

\section{Quellen}

Es sind bisher keine zeitgenössischen Belege gefunden worden.

\section{Interpretation}

Da die Kantone Neuenburg und Waadt, bzw. Bern, den Kalenderstil erst 1701 wechselten, ist die Datierung mit grosser Wahrscheinlichkeit zu korrigieren. Dieses Beben ohne Schäden ist jedoch nicht als gesichert zu werten, da der älteste Nachweis sich in der Kompilation von Bertrand aus dem Jahre 1756 befindet: "Le ving-troisième de Novembre, mille six-cent quarante-huit, on apperçut quelques secousses dans le Comté de Neufchâtel. (...) On ressentit les mêmes secousses à Yverdon.“ 
Makroseismische Parameter

\begin{tabular}{|l|l|l|l|l|l|l|}
\hline Ortschaft & Länge & Breite & $I_{\min }$ & $I_{\max }$ & $I_{\text {w }}$ & Datenqualität \\
\hline Neuchâtel & 46.99 & 6.93 & & & verspürt & sehr schwach \\
\hline Yverdon-Les-Bains & 46.78 & 6.64 & 3 & 5 & verspürt & sehr schwach \\
\hline
\end{tabular}

**

\begin{tabular}{l|l|l}
$\begin{array}{l}\text { Datum } \\
\text { Erdbebenserie } \\
\text { (Fehldatierungen) }\end{array}$ & Zeit & $\begin{array}{l}\text { Ort } \\
\text { Genfer See bis Bodensee, } \\
\text { Rheintal, etc. }\end{array}$ \\
$-1650,8$. Januar & - & - Buchs, SG \\
$-1650,6$. Mai & - & - Aesch - Basel \\
$-1650,7$. Sept. & - & - Basel \\
$-1650,10$. Sept. & -3 Uhr & - Pratteln - Basel \\
$-1650,11$. Sept. & -1 Uhr 30 & - Basel \\
$-1650,18$. Oktober & - & - Brugg, AG \\
$-1650,20$. Oktober & -12 Uhr & - Brugg, AG \\
$-1650,25$. Oktober & -11 Uhr & - Bremgarten, AG \\
$-1651,8$. Januar & - & - Basel
\end{tabular}

\section{Bewertung der Ereignisse}

Keine Erdbeben: Datierungsfehler.

\section{Korrektur}

Keine Ereignisse, sie sind alle (bis auf den 10. Sept.) um 10 Tage in den gregorianischen Kalender vorzudatieren.

- 1650,18 . Januar

- 1650, 16. Mai

- 1650, 17. Sept.

- 1650, 21. Sept.

- 1650, 28. Oktober

- 1650, 30. Oktober

- 1650, 4. November

- 1651,18 . Januar

Überlieferung u. a. in:

- Volger (1857)

- Sieberg (1940)

- $\quad$ Vogt (1987)

- MECOS-99 


\section{Quellen}

Sie sind beim Erdbeben 21. September 1650 aufgeführt.

\section{Interpretation}

Es sind zahlreiche zeitgenössische Berichte zu dieser Erdbebenserie vorhanden, die aber teilweise noch im julianischen Kalenderstil datiert sind. Das Datum 10. September 1650 ist hingegen nicht nur eine julianische Datierung, sondern mit grosser Wahrscheinlichkeit noch ein Nachtfehler. Möglicherweise handelt es sich beim 8. Januar 1651 um eine Verwechslung mit dem 8. / 18. Januar 1650.

\section{**}

\begin{tabular}{|c|c|c|}
\hline $\begin{array}{l}\text { Datum } \\
1650,21 . \text { September } \\
\text { (innerhalb der } \\
\text { Erdbebenserie vom } \\
\text { Oktober } 1649 \text { bis } \\
\text { November } 1650 \text { ) }\end{array}$ & $\begin{array}{l}\text { Zeit (UTC) } \\
3 \text { Uhr }\end{array}$ & $\begin{array}{l}\text { Ort } \\
\text { Basel (Nordostschweiz, } \\
\text { Elsass, Süddeutschland) }\end{array}$ \\
\hline
\end{tabular}

\section{Bewertung des Ereignisses}

Sicheres Ereignis.

\section{Parameter nach ECOS}

Ix: 7

\section{Überlieferung u. a. in:}

- Keferstein (1826)

- Perrey (1845)

- Volger (1857)

- Sieberg (1940)

- $\quad$ Vogt (1987)

- SisFrance

\section{Quellen}

Drucke:

- Zwinger, T. (1650): Christliche Wahrnungs- und Buoss-Predigt/Von dem Erdbidem/alss einem grossen gewaltigen Werck und Zornzeichen Gottes des Allerhöchsten. (...)

- Meyer, J. (1650): Oratio de terraemotu: ob periculossimas patriae concussiones illa A. O. R. MDCL ... .

- Schaltegger, F. (1913): Johann Heinrich Kappelers Chronik von Frauenfeld. 
- Im-Thurn, E. \& Harder, H. W. (1844): Chronik der Stadt Schaffhausen. (Umfasst die Jahre 200-1789).

- Hottinger, J. (1651): Quaestiones quaedam De Terraemotu ex Hebraeorum atque Arabum Scriptis erutae .../Sub Praesidio Dn. Joh. Henrici Hottingeri, Publicae ... defendere conabitur Johannes Zollicofferus.

Manuskripte:

- Kirchenbücher im StAZH:

- Benken: E III 013.1

- Brütten: E III 017.1

- Stammheim: E III 0117.2

- Meilen: E III 075.2

- Kilchberg: E III 062.2

- Stäfa: E III 0115.3

- Stiftsarchiv St. Gallen (StiASG):

- $\quad$ Tagebuch des Abtes Pius 1650-1653: Bd 262 B.

- Stiftsarchiv Einsiedeln (StiAEi):

- Diarium des Abtes Placidus und anderer, 1636-1656: A. HB 101.

- Schlossbibliothek Wildegg (SbWi):

- Stumpfchronik (Ausgabe 1586), handschriftlicher Eintrag von Hans Friedrich Effinger zu 2 Erdstössen am 21. (11.) September und 28. (18.) Oktober 1650.

- Stadtarchiv Stein am Rhein (StASaR):

- Isaak Vetter (1692-1747), Geschichten der Stadt Stein, 7 Bde, Bü 47-53: Bü 49, Bd III 1600-1699. (Kompilation)

\section{Interpretation}

Vom Oktober 1649 bis anfangs November 1650 wurden über 20 Erdbeben im Gebiet der Nordostschweiz, des Elsasses und Süddeutschlands verspürt, die dank zahlreicher zeitgenössischer Quellen gesichert nachgewiesen, aber grösstenteils noch nicht lokalisiert sind. Das grösste Beben fand am 21. September 1650, greg. Kalender, statt und verursachte Schäden in Basel. Sie fanden in der Öffentlichkeit grosse Beachtung, teilweise wurden von den Behörden religiöse Gegenmassnahmen ergriffen, wie zum Beispiel das Abhalten von Busstagen.

Der Theologe Zwinger beschreibt die Situation in Basel folgendermassen: „... demnach in disem September oder Herbstmonat/insonderheit nächst-verwichenen Mitwochen (11. Sept. jul.) des Morgens gegen 5. Uhren/mit einem erschrockenlichen Erdbidem/ welcher unser gantze Statt/und in derselbigen alle Häuser/und alle auch die höchsten Thürne/mit grossem gewalt und krachen der-gestalten erschüttet hat/dass auch die Glocken in den Kirch-thürnen/und ebengrad in unseren Münster-thürnen/wie auch in etlichen Häuseren/durch ihre bewegung sich hören und mercken lassen/auch etliche Ziegel und Camin von den Tächeren mit grossem braschlen auff die Erden herunder gefallen/welcher auch mit etlichen anderen Erdbimen folgende tage begläitet worden/uns erschrecket und heim-gesucht hat (S. 4-5)“. Bestätigt von J. M. Meyer (1650), der die entstandenen Schäden mit „aliquot caminis [einigen Kaminen]“ umschreibt. 
Gemäss anderen zeitgenössischen Quellen, wie zum Beispiel dem Diarum des St. Galler Abtes Pius oder den Pfarrbüchern von Brütten und Benken, ZH, ist das Erdbeben mit grösserer Wahrscheinlichkeit auf 4 Uhr (3 UTC) festzulegen.

Bei einer künftigen Auswertung muss den einzelnen Datierungen besondere Beachtung geschenkt werden, um Verdoppelungen der Ereignisse auszuschliessen.

\section{Makroseismische Parameter}

\begin{tabular}{|l|l|l|l|l|l|l|}
\hline Ortschaft & Länge & Breite & $I_{\min }$ & $I_{\max }$ & $I_{\text {w }}$ & Datenqualität \\
\hline Benken ZH & 47.65 & 8.65 & & & verspürt & sehr schwach \\
\hline Niederhasli & 47.48 & 8.48 & & & verspürt & sehr schwach \\
\hline Wildegg & 47.41 & 8.16 & & & verspürt & sehr schwach \\
\hline Einsiedeln & 47.13 & 8.74 & & & verspürt & sehr schwach \\
\hline Basel & 47.56 & 7.59 & 6 & 7 & 7 & mässig \\
\hline Zürich & 47.37 & 8.54 & 5 & 6 & 5 & sehr schwach \\
\hline Brütten & 47.47 & 8.68 & 4 & 5 & 5 & sehr schwach \\
\hline Stäfa & 47.25 & 8.73 & 4 & 5 & 5 & sehr schwach \\
\hline Kilchberg ZH & 47.32 & 8.54 & 4 & 5 & 5 & sehr schwach \\
\hline Wetzikon ZH & 47.33 & 8.8 & 3 & 4 & 4 & sehr schwach \\
\hline Schaffhausen & 47.7 & 8.64 & 4 & 5 & 4 & sehr schwach \\
\hline St. Gallen & 47.43 & 9.4 & 4 & 5 & 4 & sehr schwach \\
\hline Unterstammheim & 47.64 & 8.79 & 4 & 5 & 4 & sehr schwach \\
\hline Frauenfeld & 47.56 & 8.89 & 3 & 4 & 3 & sehr schwach \\
\hline
\end{tabular}

\section{Bemerkungen}

Die gleichzeitige Verwendung des gregorianischen und des julianischen Kalenderstils führte bei diesem Erdbebenschwarm zu Verdoppelungen von Ereignissen, von denen bisher erst einige identifiziert werden konnten, diese identifizierten Fehldatierungen sind in der vorhergehenden Tabelle zur Erdbebenserie 1650 aufgeführt.

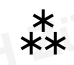

Datum

1651, 7. Dezember
Zeit

$15 \mathrm{Uhr}$
Ort

Genève

\section{Bewertung des Ereignisses}

Kein Erdbeben: Datierungsfehler. 


\section{Korrektur}

1651, 17. Dezember

\section{Überlieferung}

- Spon (1682)

- Scheuchzer (1706)

- Volger (1857)

\section{Interpretation}

Spon (1682, S. 245) verzeichnet ein leichtes Erdbeben:

1651. 7. Dezember: „Le mois suivant (Nov.) il y eut un tremblement de terre sur le soir, qui ne fit point de mal.“ (Am 7. Dezember 1651 gab es abends ein Erdbeben, welches keinen Schaden verursachte.) Das calvinistische Genf änderte seinen Datierungsstil erst 1701, somit ist Spons Angabe um 10 Tage zu verschieben.

\section{Bemerkungen}

Vgl. im ECOS-09 das Erdbeben vom 17. Dezember 1651.

\section{**}

\begin{tabular}{l|l|l} 
Datum & Zeit & Ort \\
-1652, 2. Februar & - & - Sax \\
$-1652,4$. Februar & - & - \\
-1652, 14. Februar & - & - Zürich
\end{tabular}

\section{Bewertung des Ereignisses}

Keine Erdbeben: Datierungsfehler.

\section{Korrektur}

1652, 11. Februar

\section{Überlieferung u. a. in:}

- Scheuchzer (1706)

- Lindiner (1711)

- Bertrand (1756)

- Volger (1857)

- MECOS-99

\section{Zeitgenössische Quelle:}

- Schaltegger (1913): Kappeler 


\section{Interpretation}

In den Kompilationen kursieren für den 2. und den 4. Februar 1652 mehrere Hinweise aus julianisch datierenden Orten wie Zürich, Basel, Schaffhausen, Sax und Werdenberg. Der einzige zeitgenössische Beleg stammt von Kappeler aus Frauenfeld (die Gemeine Herrschaft Thurgau datierte ebenfalls noch julianisch), er setzte ein Beben auf die Zeit vor dem 2. Februar an: „[70] Anno 1652 nechst vor der alten Liechtmess ward widerum ein Erdbedem zu Zürich Basel und Schaaffussen gespürt.“ Damit hat es spätestens am 1./11. Februar stattgefunden.

Es konnten weder zeitgenössische Hinweise auf die Intensität noch auf eine Erdbebenserie gefunden werden.

\section{Bemerkungen}

Vgl. das Erdbeben vom 11. Februar 1652 in der Nordschweiz.

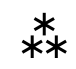

\begin{tabular}{|l|l|l}
\hline Datum & Zeit (UTC) & Ort \\
1 1652, 11. Februar & - & Nordschweiz
\end{tabular}

\section{Bewertung des Ereignisses}

Unsicheres Ereignis.

\section{Parameter nach ECOS}

Ix: verspürt

\section{Überlieferung}

- Scheuchzer (1706)

- Lindiner (1711)

- Bertrand (1756)

- Volger (1856)

- Kopp (1861)

- Tribolet (1876)

\section{Quelle}

- Schaltegger (1913): Chronik von Johann Heinrich Kappeler von Frauenfeld. Nach dem im Bürgerarchiv Frauenfeld liegenden Manuskript (M. 15. S. 297-347).

\section{Interpretation}

Zwar wird in der Zürcher Kompilation von Lindiner das Beben julianisch auf den 4. Februar 1652 datiert, doch der zeitgenössische Kappeler aus Frauenfeld widerspricht dem mit einem kleinen Interpretationsspielraum in julianischer Datierung: „Kurz vor dem 
2. Februar: [70] Anno 1652 nechst vor der alten Liechtmess ward widerum ein Erdbedem zu Zürich Basel und Schaaffussen gespürt." Damit ist der 1. bzw. der 11. Februar (greg.) das wahrscheinlichste Datum. Bertrand (1756) verwendet eine auffallend ähnliche Formulierung wie Kappeler. Kompilatorische Andeutungen auf eine mögliche Erdbebenserie konnten bisher nicht verifiziert werden. Der Sitepunkt "Neuchâtel“ stammt aus den unzuverlässigen Kompilationen von Tribolet und Kopp, sodass er zu vernachlässigen ist. Damit ist mit „Nordschweiz“ die Lokalisierung genauer umschrieben. Das Ereignis ist aufgrund der jetzigen Quellenlage noch als ungesichert zu werten. Es gibt keine Hinweise auf Schäden.

Makroseismische Parameter

\begin{tabular}{|l|l|l|l|l|l|l|}
\hline Ortschaft & Länge & Breite & $I_{\min }$ & $I_{\max }$ & $I_{\text {w }}$ & Datenqualität \\
\hline Basel & 47.56 & 7.59 & & & verspürt & sehr schwach \\
\hline Zürich & 47.37 & 8.54 & & & verspürt & sehr schwach \\
\hline Schaffhausen & 47.7 & 8.64 & & & verspürt & sehr schwach \\
\hline Neuchâtel & 46.99 & 6.93 & & & verspürt & sehr schwach \\
\hline
\end{tabular}

\section{$\stackrel{*}{* *}$}

\section{Datum}

1652, 19. August

\section{Zeit}

$-$

\section{Ort}

\section{Bewertung des Ereignisses}

Kein Erdbeben: Datierungsfehler.

\section{Korrektur}

29. August 1652, kein Lokalisierungsvorschlag

\section{Überlieferung}

- Scheuchzer (1718)

- Keferstein (1826): S. 298: „1652. In den Schweizer Alpen Erdstösse den ... 19. August .... ."

- Volger (1857): „Erdbeben zu Eglisau am Rhein“

- MECOS-99

\section{Quellen}

Es sind bisher keine Originalquellen nachweisbar.

\section{Interpretation}

Da die Kompilationen auf eine Herkunft der Nachricht aus reformierten Kantonen deuten, so ist die Datierung eher auf den 29. August zu fixieren. Volger lokalisiert das Ereig- 
nis in Eglisau, ZH, was noch nicht bestätigt werden kann. Bei dieser knappen Datenlage ist dieses Erdbeben noch nicht weiter bestimmbar.

\section{Bemerkungen}

Vgl. im ECOS-09 das Erdbeben vom 29. August 1652.

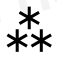

\section{Datum}

- 1652, 13. Oktober

- 1652, 23. Oktober
Zeit (UTC)
Ort

- Niederurnen-Näfels, GL

- Niederurnen-Näfels, GL

\section{Bewertung des Ereignisses}

Kein Erdbeben: Datierungsfehler und Lokalisierungsfehler.

\section{Korrektur}

1652, 23. Oktober, Rapperswil, SG

\section{Überlieferung u. a. in:}

- Scheuchzer (1706)

- Keferstein (1826)

- Volger (1857)

Zeitgenössische Quelle:

- Pfarrbuch Meilen, StAZH: E III 075.2

\section{Interpretation}

Das zeitgenössische Pfarrbuch aus dem reformierten Meilen, Kanton Zürich, datiert im julianischen Kalenderstil, sodass es und die aufgeführten Kompilatoren um 10 Tage auf den 23. Oktober zu korrigieren sind: „Den 13. Octob. hatt sich ein erdbidem merken lassen des Mittags umb 12 Uhren. ward gespürt zuo Stäfen, Raperschwyl, im Land Glarus (et caetera). So hab ich auch von Bilgern, von Sultz, auss dem Obern Elsass gehört, das an selben orden auch einer sich mercken lassen den tag aber unt stund, konntend sy eigentlich nit sagen."

\section{Bemerkungen}

Vgl. das Erdbeben vom 23. Oktober 1652.

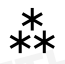




\section{Datum \\ Zeit \\ Ort \\ 1652, 23. Oktober \\ $11 \mathrm{Uhr}$ \\ Nicht lokalisierbar (Rapperswil, SG)}

\section{Bewertung des Ereignisses}

Unsicher.

\section{Parameter nach ECOS}

Ix: verspürt

\section{Überlieferung}

- Scheuchzer (1706)

- Keferstein (1826)

- $\operatorname{Volger}(1857)$

\section{Quelle}

_ Pfarrbuch Meilen, StAZH: E III 075.2

\section{Interpretation}

Das zeitgenössische Pfarrbuch aus dem reformierten Meilen, Kanton Zürich, datiert im julianischen Kalenderstil, sodass es und die aufgeführten Kompilatoren um 10 Tage auf den 23. Oktober zu korrigieren sind: „Den 13. Octob. hatt sich ein erdbidem merken lassen des Mittags umb 12 Uhren. ward gespürt zuo Stäfen, Raperschwyl, im Land Glarus (et caetera). So hab ich auch von Bilgern, von Sultz, auss dem Obern Elsass gehört, das an selben orden auch einer sich mercken lassen den tag aber unt stund, konntend sy eigentlich nit sagen."

Mit nur einer zeitgenössischen Quelle kann die Ausdehnung des makroseismischen Feldes nur mit einer gewissen Wahrscheinlichkeit mit „vom Kanton Zürich bis in den Kanton Glarus“ umschrieben werden, unter Umständen auch bis ins Elsass. Die Kompilatoren scheinen teilweise von der Aussage des Pfarrbuches abhängig zu sein. Es gibt keine Hinweise auf Schäden.

\section{Makroseismische Parameter}

\begin{tabular}{|l|l|l|l|l|l|l|}
\hline Ortschaft & Länge & Breite & $I_{\min }$ & $I_{\max }$ & $I_{\text {w }}$ & Datenqualität \\
\hline Rapperswil SG & 47.23 & 8.83 & & & verspürt & sehr schwach \\
\hline Stäfa & 47.25 & 8.73 & & & verspürt & sehr schwach \\
\hline Meilen & 47.28 & 8.65 & & & verspürt & sehr schwach \\
\hline
\end{tabular}

\section{$\stackrel{*}{* *}$}




\begin{tabular}{l|l|l} 
Datum & Zeit & Ort \\
-1655 & - & - Nicht lokalisierbar (Lausanne) \\
$-(1660$ & - & - Nicht lokalisierbar (Lausanne) \\
$-1665)$ & - & - Nicht lokalisierbar (Lausanne)
\end{tabular}

\section{Bewertung des Ereignisses}

Sehr unsichere Erdbeben.

\section{Parameter nach ECOS für 1655}

Ix: 6

\section{Überlieferung}

- Heinzmann (1795)

- $\quad$ Beer, de, G. R. (1953): Text, S. 207-208:

[207] Gilbert Burnet, ${ }^{27}$ évêque de Salisbury, passa par le Pays de Vaud en 1685: „La ville de Lausanne, dit-il, est située sur trois collines de sorte que ce n'est que montées et descentes très raides, surtout du côté de l'église qui est une très noble bâtisse. II y a environ trente ans depuis qu'un tremblement de terre fendit le mur méridional du transept de haut en bas, laissant une [208] fente de plus d'un pied de largeur [30cm]. Dix ans après, une autre secousse reboucha l'ouverture dont on ne voit plus que l'emplacement.“ [...]

- Keyssler, Johann, Georg (1751, S. 1349):

Lausanne ... . In der Mauer der Hauptkirche wird der Riss gezeiget, welchen im Jahre 1634 (sic!) ein Erdbeben verursachet hat, von solcher Weite, dass ein Mann dadurch kriechen konnte. Der berühmte alte Professor Pictet erzählete oftmals, dass er in seiner Jugend, wenn er auf dem Kirchhofe mit andern Knaben gespielet, bisweilen seinen Mantel hinein gelegt habe. Vor dreyssig Jahren ist durch ein anders Erdbeben alles wieder genau zusammen gefügt, und die schmale übrige Ritze, welche nunmehr kaum eines Daumens dick, mit Kalke ausgefüllet worden. (...)

- Brief mit archäologischen Plänen und telephonische Auskünfte von W. Stöckli vom „Atelier d'archéologie médiévale“ in Moudon, August 2009: In der Kathedrale von Lausanne sind mehrere Schäden nachgewiesen, die auf ein Erdbeben deuten. Dabei handelt es sich vor allem um Risse in einigen Pfeilern, die aber nicht datierbar sind.

\section{Interpretation}

Es gibt nur zwei und unterschiedlich datierende späte Quellen, die eine toposartige Darstellung zweier Erdbebenschäden an der Lausanner Kathedrale geben $(1634,1660$, 1655, 1665). Sie werden aber von archäologischen Befunden an einigen Pfeilern der Kathedrale gestützt, die Risse, wie sie durch ein Erdbeben entstehen können, auf-

27 Gilbert Burnet (1643-1715), voir D.N.B.; auteur de „Some Letters containing what seemed most remarkable in Switzerland, Italy etc.“, Amsterdam, 1686. 
weisen. Da im 17. Jahrhundert die Schriftlichkeit bereits gut entwickelt war, müssten allerdings mehr Schriftquellen zu einem Schadenbeben vorhanden sein. Der Widerspruch liesse sich auflösen, wenn es sich um eine Verwechslung mit den Erdbeben vom 11.-14. März 1584 handeln würde, wo in Lausanne am 11. März 1584 nachweislich Schäden entstanden sind. Trotzdem werden diese Meldungen vorläufig unter ihrem eigenen Datum aufgeführt und als „sehr unsicher“ bewertet.

\section{Makroseismische Parameter}

1655

\begin{tabular}{|l|l|l|l|l|l|l|}
\hline Ortschaft & Länge & Breite & $I_{\min }$ & $I_{\max }$ & $I_{\text {w }}$ & Datenqualität \\
\hline Lausanne & 46.52 & 6.63 & 5 & 6 & 6 & sehr schwach \\
\hline
\end{tabular}

1660

\begin{tabular}{|l|l|l|l|l|l|l|}
\hline Ortschaft & Länge & Breite & $I_{\min }$ & $I_{\max }$ & $I_{\text {w }}$ & Datenqualität \\
\hline Lausanne & 46.52 & 6.63 & 5 & 6 & 6 & sehr schwach \\
\hline
\end{tabular}

1665

\begin{tabular}{|l|l|l|l|l|l|l|}
\hline Ortschaft & Länge & Breite & $I_{\min }$ & $I_{\max }$ & $I_{w}$ & Datenqualität \\
\hline Lausanne & 46.52 & 6.63 & 5 & 6 & 6 & sehr schwach \\
\hline
\end{tabular}

\section{Bemerkungen}

Vgl. die Erdbeben vom 11.-14. März 1584.

\section{$\stackrel{*}{* *}$}

Datum

1655, März-April
Zeit -
Ort

Tübingen, D

\section{Bewertung der Ereignisse}

Sichere Serie von Erdbeben mit sehr unsicherer Tages-Datierung.

\section{Parameter nach ECOS}

Ix: keine Wertung

\section{Überlieferung u. a. in:}

- Isaak Vetter [1692-1747], (Stadtarchiv Stein am Rhein, Bü 47-53)

- Langenbeck (1892)

- $\quad$ Giessberger (1922) 
- Sieberg (1940)

- Rüttener (1995)

- MECOS-99

- $\quad$ CENEC, 2009

- SisFrance (2009)

Zeitgenössische Quelle:

Burgauer (StASH, o. Sign.)

\section{Interpretation}

Diese Erdbebenserie, die auch die Schweiz betroffen hat, lässt sich noch nicht abschliessend beurteilen. In älteren Kompilationen wird der Beginn einer Erdbebenserie auf den 19. oder 29. März 1655 festgesetzt. Es ist dann Sieberg (1940), der das Datum 11. April nennt.

Der zeitgenössische Johann Burgauer aus dem protestantischen Schaffhausen führt hingegen nur den 29. März und den 2. April auf, die gregorianisch auf den 8. und 12. April zu datieren sind. Isaak Vetters Arbeit „Geschichten der Stadt Stein“ aus dem 18. Jahrhundert wiederum zitiert ein Manuskript eines gewissen Stöcklin, wo der 19., 24. und 30. März genannt werden, der Kalenderstil ist auch hier mit grosser Wahrscheinlichkeit julianisch.

Im deutschen Katalog CENEC werden die Daten 19. März, 30. März, 3. und 11. April genannt und alle in Tübingen lokalisiert. Die Epizentral-Intensität wird für den 19. März und den 3. April auf 6-6.5 geschätzt. Während SisFrance auf den 8. April datiert und ebenfalls in Tübingen lokalisiert, und zwar mit einer Intensität 5.

Erst zusätzliche zeitgenössische Dokumente werden erlauben, den Kalenderstil zu bestimmen und damit den Verlauf und die maximale Intensität dieser Erdbeben-Serie zuverlässig zu beschreiben.

\section{Bemerkungen}

Vgl. im ECOS-09 die Erdbeben vom 19. März, 30. März, 3. April und 11. April 1655 aus CENEC.

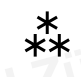
Datum
Zeit
Ort
- 1656, 23. Februar
- 1656, 2. März
- Basel
- Blauen-Ettingen, BL

\section{Bewertung des Ereignisses}

Keine Erdbeben: Datierungsfehler. 


\section{Korrektur}

1656, 4. März, Basel

\section{Überlieferung u. a. in:}

- Scheuchzer (1706)

- Bertrand (1756)

- Volger (1857)

- MECOS-99

\section{Interpretation}

Bei Bertrand wird nur vom Februar gesprochen (1656: Dans le mois de Février, mille sixcent cinquante-six, Bâle \& tous ses environs, furent exposés, dans une nuit, à trois tremblemens différens); während bei Volger mit „der 23. Februar alten Kalenders“ [4. März] datiert wird, was im MECOS-99 wiederum ohne entsprechende Korrektur übernommen worden war. Originalquellen und Herkunft der Lokalisierung sind bisher unbekannt. Damit handelt es sich auch beim korrigierten Datum um ein sehr unsicheres Ereignis; es werden keine Schäden erwähnt.

\section{Bemerkungen}

Vgl. im ECOS-09 das Erdbeben vom 4. März 1656.

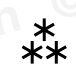

\begin{tabular}{l|l|l} 
Datum & Zeit & Ort \\
$1661,9$. Januar & $21 \mathrm{Uhr}$ & Glarus
\end{tabular}

\section{Bewertung des Ereignisses}

Kein Erdbeben: Datierungsfehler

\section{Korrektur}

1661, 18. Januar, 22 Uhr, Meilen

\section{Überlieferung u. a. in:}

- Tschudi (1714)

- Isaak Vetter [1692-1747], Manuskript, erste Hälfte 18. Jh. (StASaR: Bü 47-53)

- Bertrand (1756)

- Volger (1857)

- Montandon (1942-43)

- MECOS-99

Zeitgenössische Quelle:

Pfarrbuch Meilen: StAZH: E III 075.2: Taufen, Ehen, Tote, 1617-1712. 


\section{Interpretation}

Das zeitgenössische Pfarrbuch von Meilen hält im julianischen Kalenderstil ein Erdbeben für den Januar 1661 fest: „Den .8. vor Mittnacht, umb 11. uhren, wurd ein grosser, starker Ertbidem gespührt, in der Stat, auf dem Land, und an anderen Ohrten“. Johann Heinrich Tschudi, der sich auf eine unbekannte Quelle stützt, bestätigt die Meldung mit „8. Jenner ... 10 Uhr abends“. Isaak Vetter von Stein am Rhein zitiert einen Kalender (p. 426r) [Bemerkung rechter Rand:] Ao. 1661, Grimm Calender ... „den 8. Jan. Nachts zwüschend 10. und 11. Uhren war ein Erdbidem.“

Es sind erst Bertrand und seine Kopisten, die auch den 9. Januar, jul. Datierung, nennen. Damit hat das Beben mit einer gewissen Wahrscheinlichkeit zwischen 22 und 23 Uhr Ortszeit, oder um 22 Uhr UTC am 18. Januar 1661 (greg.) stattgefunden. Es handelt sich bei der Datierung 9. Januar also um eine Kombination von Nacht- und Kalenderfehler, die auf den 18. Januar zu korrigieren ist.

\section{Bemerkungen}

Vgl. das Erdbeben vom 18. Januar 1661 in Meilen, ZH.

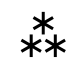

\begin{tabular}{l|l|l} 
Datum & Zeit & Ort \\
1661, 10. Februar & - & Glarus
\end{tabular}

\section{Bewertung des Ereignisses}

Kein Erdbeben: Datierungsfehler.

\section{Korrektur}

1661, 20. Februar

\section{Überlieferung}

- Trümpi, Christoph (1774)

- MECOS-99

\section{Interpretation}

Bisher ist dieses Erdbebendatum nur aus einer Kompilation bekannt, der GlarnerChronik von Christoph Trümpi. Da der Kanton Glarus erst im Jahr 1701 den Kalender wechselte, müssen für eine gregorianische Datierung zehn Tage addiert werden: „Vom 10. Hornung 1661. in unserm Land ein empfindlicher Erdbidem angemerket.“ (S. 375), also 20. Februar. Mit einem einzigen kompilatorischen Hinweis ist auch das korrigierte Datum als „unsicher“ zu werten. 


\section{Bemerkungen}

Vgl. das Erdbeben vom 20. Februar 1661, (Kanton) Glarus.

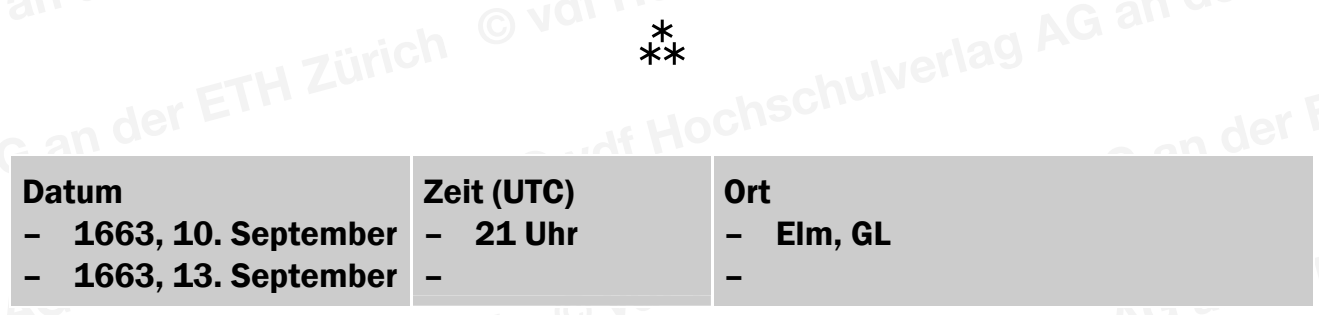

\section{Bewertung der Ereignisse}

Keine Erdbeben: Datierungsfehler.

\section{Korrektur}

1663, 20. und 23. September, Kanton Glarus

\section{Überlieferung u. a. in:}

- Tschudi (1714)

- Bertrand (1756)

- Trümpi, Christoph (1774)

- Volger (1857)

- Montandon (1942-43)

- Gils, Van \& Leydecker (1991)

- MECOS-99

\section{Interpretation}

Tschudi schreibt 1714 aufgrund einer ungenannten Quelle von „Erdbidem ..., 10. und 13. September 1663. Den 10 Tag Herbstmonat Abends um 10 Uhr ist abermals in diesem Land/ein zimlich starcker Erdbidem/und folgenden Sonntags/den 13. diss Monats/ein anderer vermercket worden. “ Die Datierungen sind wahrscheinlich dem Glarner Gebrauch entsprechend julianisch und müssen deshalb um 10 Tage korrigiert werden. Zeitgenössische Quellen zur Bestätigung fehlen hingegen. Ebenso ist die Herkunft der Lokalisierung „Elm“ in Van Gils \& Leydecker unbekannt.

\section{Bemerkungen}

Vgl. im ECOS-09 die Erdbeben vom 20. und 23. September 1663.

$$
\text { ** }
$$




\section{Datum \\ Zeit \\ Ort \\ 1665, 3. März \\ $-$

\section{Bewertung des Ereignisses}

Kein Erdbeben: Datierungsfehler.

\section{Korrektur}

1665, 13. März in Einsiedeln, Sz.

\section{Überlieferung}

- Trümpi (1774)

- Dettling, Schwyzerischer Geschichtskalender (1921): „1665, 13. März. In Einsiedeln verspürt man ein starkes Erdbeben.“

- MECOS-99

\section{Quelle}

- Heiligensetzer (2001), S. 123: Vom reformierten Pfarrer Alexander Bösch, Pfarrer in Krummenau und Kappel, ohne Tagesdatierung: „Anno 1665 sahe mann Cometsternen und gspürte Erdbidem.“

\section{Interpretation}

Bisher war dieses Erdbeben aus zwei Kompilationen bekannt, und zwar aus der GlarnerChronik von Christoph Trümpi und aus dem Schwyzer Geschichtskalender. Da der Kanton Glarus erst im Jahr 1701 den Kalender wechselte, müssen, entsprechend der sicherlich katholischen Quelle aus Einsiedeln, für eine gregorianische Datierung zehn Tage addiert werden: „1665, 13. März. In Einsiedeln verspürt man ein starkes Erdbeben.“ Ein zeitgenössischer Hinweis auf ein Beben im Jahr 1665 wurde in einem Toggenburger Familienbuch gefunden, doch ohne Tagesangabe, womit er wenig aussagekräftig ist.

\section{Bemerkungen}

Vgl. im ECOS-09 das Erdbeben vom 13. März 1665.

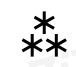

\begin{tabular}{l|l|l} 
Datum & Zeit & Ort \\
$1665,31$. März & - & Neuchâtel
\end{tabular}

\section{Bewertung des Ereignisses}

Kein Erdbeben: Datierungsfehler. 


\section{Korrektur}

1665, 10. April

\section{Überlieferung}

- Bertrand (1756)

- Volger (1857)

\section{Interpretation}

Bisher konnten keine zeitgenössischen Quellen nachgewiesen werden, die Kompilationen lokalisieren das Ereignis in Neuenburg, sodass beim 31. März eine julianische Datierung angenommen werden muss, die entsprechend um 10 Tage zu korrigieren ist. Es handelt sich auch bei dem umdatierten Erdbeben um ein sehr unsicheres Ereignis.

\section{Bemerkungen}

Vgl. das Erdbeben vom 10. April 1665.

\begin{tabular}{l|l|l|}
\multicolumn{3}{c}{$\begin{array}{l}* \\
* *\end{array}$} \\
Datum & Zeit & Ort \\
1666, 1. März & - & Glarus
\end{tabular}

\section{Bewertung des Ereignisses}

Kein Erdbeben: Datierungsfehler.

\section{Korrektur}

1666, 11. März

\section{Überlieferung}

- Tschudi (1714)

- Trümpi (1774)

\section{Interpretation}

Bisher konnten keine zeitgenössischen Quellen nachgewiesen werden, die Kompilationen lokalisieren das Ereignis aber in Glarus, sodass beim 31. März eine julianische Datierung angenommen werden muss, die entsprechend um 10 Tage zu korrigieren ist.

\section{Bemerkungen}

Vgl. das Erdbeben vom 11. März 1666.

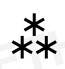




\begin{tabular}{l|l|l}
\hline Datum & Zeit & Ort \\
$-1666,1$. September & - & - Bodensee \\
$-1666,11$. September & - & - Arbon, TG
\end{tabular}

\section{Bewertung des Ereignisses}

Kein Erdbeben: Datierungsfehler, möglicher handelt es sich um eine Flutwelle durch eine subaquatische Rutschung.

\section{Korrektur}

1666, 11. September

\section{Überlieferung u. a. in:}

- Scheuchzer (1706)

- Bertrand (1756)

- $\quad$ Hoff (1840-1841)

- Volger (1857)

- MECOS-99

\section{Dürresommer:}

- Pfister, Witterungsdatei Climhist (1984)

Quellen:

- Telefonische und briefliche Nachfragen in mehreren Archiven der Umgebung ergaben keine Hinweise auf ein Erdbeben oder auf eine Überschwemmung, so zum Beispiel in den Tauf-, Ehe- und Totenregistern der evangelischen und katholischen Kirchengemeinde Arbon (StATG MF 995275 bzw. kath. StATG MF 995257), gemäss E-Mail des Archivars Spalinger vom Staatsarchiv des Kantons Thurgau, 30.10.08; ebenso fehlen Hinweise im Staatsarchiv und im Stiftsarchiv von St. Gallen und in den Gemeindearchiven von Arbon, Romanshorn, Rorschach und Rorschacherberg.

- Keine zeitgenössischen Quellen nachweisbar.

\section{Interpretation}

Das Gebiet des heutigen Kanton Thurgau war zu jenem Zeitpunkt Gemeine Herrschaft der Alten Eidgenossenschaft und wechselte den Kalenderstil erst 1701, sodass diese Beobachtung in den gregorianischen Kalender vorzudatieren ist.

Doch es bleibt ein weiterer Punkt ungeklärt. Bisher liess sich dieses Ereignis, ein angebliches Erdbeben bei Arbon und kurzzeitige Überschwemmung des Ufers am 1., bzw. 11. greg., September 1666 nur bei den Kompilatoren Scheuchzer (1706/1746) und, von inm abhängend, Bertrand (1756) in ähnlicher Weise nachweisen: „A. 1666. spürte man Erdbeben. Den 1. Sept. zu Arbon am Boden-See und umliegenden Orten. (...)“. Wobei Bertrand über zusätzliche Informationen verfügt zu haben scheint: „En mille six-cent soixante-six, le premier de Septembre, il y eut un tremblement de terre à Arbon, ancienne Ville sur le Lac de Constance. Les eaux du Lac s'avancèrent sur le rivage de plus de 25 à 30 pieds (ungefähr 7,5 bis $9 \mathrm{~m}$ ), \& se retirèrent subitement.“ 
Da zeitgenössische Hinweise aus der weiteren Umgebung zu einem Erdbeben fehlen, so gilt es, auch die Niederschlagsverhältnisse zu diesem Zeitpunkt zu überprüfen. In der Witterungsdatei Climhist, Pfister (1984 und 2009), die eine siebenteilige NiederschlagsSkala aufweist $\left({ }^{+}+3^{\prime \prime}\right.$ bis $\left.{ }-3^{\prime \prime}\right)$, sind folgende Niederschlags-Monatswerte für die Schweiz aufgeführt:

1666: Jan: 0, Feb: -1, März: +1, Apr: 0, Mai: -2, Jun: 0, Jul: -3, Aug: -1, Sep: -1 .

Damit handelte es sich beim Sommer 1666 offensichtlich um einen Dürresommer, der einen tiefen Wasserstand zur Folge gehabt haben muss. Wenn man nun wegen der fehlenden zeitgenössischen Beobachtungen nicht direkt auf eine Falschmeldung schliessen will, so kann auch spekuliert werden, dass es sich hier vielleicht um eine subaquatische Rutschung mit nachfolgender Flutwelle gehandelt haben könnte, vielleicht im Bereich der Mündung der Aach.

\section{Bemerkungen}

Vgl. die Erdbeben mit subaquatischen Rutschungen vom 11. März 1584, vom 18. September 1601; und die Rutschung vom 24. September 1687 im Muotadelta, ohne vorhergehendes Erdbeben, dafür mit extremen Niederschlägen.

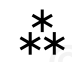

\begin{tabular}{|l|l|l}
\hline Datum & Zeit & Ort \\
\hline $1668,20$. April & $14 \mathrm{Uhr}$ & Glarus
\end{tabular}

\section{Bewertung des Ereignisses}

Kein Erdbeben: Datierungsfehler

\section{Korrektur}

1668, 30. April

\section{Überlieferung u. a. in:}

- Scheuchzer $(1706,1746)$

- Bertrand (1756)

- Trümpi (1774)

- Volger (1857)

- MECOS-99

Nicht vorhanden in:

Candreia (1905): Zur Chronik der Erdbeben in Graubünden. 


\section{Interpretation}

Scheuchzer zitiert Tschudi: „A. 1668. den 20. April Abends zwischen 3. und 4. Uhr ist in diesem Lande allenthalben ein starckes Erdbeben mit grossem Zittern und Beben gehört und vermercket worden." Da Glarus aber erst 1701 vom julianischen zum gregorianischen Kalender gewechselt hat, muss zumindest die Datierung korrigiert werden. Im weiteren sind aber bisher keine Originalquellen aus Glarus oder Umgebung gefunden worden, womit auch die Datierung 30. April als „unsicher" gewertet werden muss.

\section{Bemerkungen}

Vgl. im ECOS-09 das Erdbeben vom 30. April 1668.

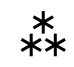

\begin{tabular}{l|l|l}
\hline Datum & Zeit (UTC) & Ort \\
\hline $1669,30$. September & 12 Uhr 45 & Strasbourg, F
\end{tabular}

\section{Bewertung des Ereignisses}

Kein Erdbeben: Datierungsfehler.

\section{Korrektur}

1669, 10. Oktober

\section{Überlieferung u. a. in:}

- Langenbeck (1892)

- Giessberger (1922)

- Sieberg (1940)

- Rüttener (1995)

- MECOS-99

- SisFrance (8. August 2009)

\section{Interpretation}

Es zirkulieren drei verschiedene Datierungen: 20. und 30. September und 10. Oktober. Da jedoch eine zeitgenössische Quelle aus Strassburg den 30. September nennt, so ist dies als julianische Datierung zu werten, da in dieser Stadt erst ab 1682 gregorianisch datiert wurde, und auf den 10. Oktober umzudatieren. Im Bistum Strassburg hingegen war der Kalender bereits 1583 gewechselt worden.

\section{Bemerkungen}

Vgl. das Erdbeben vom 10. Oktober 1669.

\section{***}



Datum
Zeit
Ort
1669, 10. Oktober
12 Uhr 45
Plain de Basse-Alsace, $\mathbf{F}$

\section{Bewertung des Ereignisses}

Sicheres Erdbeben.

\section{Parameter nach ECOS}

Ix: 6

\section{Überlieferung u. a. in:}

- Volger (1857)

- Langenbeck (1892)

- Giessberger (1922)

- Sieberg (1940)

- Rüttener (1995)

- MECOS-99

- SisFrance (8. August 2009)

\section{Quellen}

Vgl. die Bibliografie von SisFrance

\section{Interpretation}

Wegen der Kalenderproblematik werden in den Kompilationen drei verschiedene Daten genannt, eventuell gab es sogar noch am folgenden Tag ein Nachbeben. Dieses französische Hauptbeben konnte im Rahmen des Projektes jedoch nicht vertieft untersucht werden, sodass hier der On-line-Katalog SisFrance (8. August 2009) zitiert wird. Er stützt sich auf zwei zeitgenössische Quellen und vier Kompilationen. Obwohl nur eine dieser zeitgenössischen Quellen Gegenstände aufzählt, die in den Häusern von Strassburg auf den Boden gefallen seien, wird dieses Beben hier mit „sicher“ und der Intensität Ix $=6$ bewertet. Der Basler Sitepoint stützt sich auf Volger (1857) und seine Kompilatoren ab.

\section{Makroseismische Parameter (ohne französische Parameter)}

\begin{tabular}{|l|l|l|l|l|l|l|}
\hline Ortschaft & Länge & Breite & $I_{\min }$ & $I_{\max }$ & $I_{w}$ & Datenqualität \\
\hline Basel & 47.56 & 7.59 & & & verspürt & sehr schwach \\
\hline
\end{tabular}

\section{Bemerkungen}

Vgl. SisFrance, 10. (und 11.) Oktober 1669. 


\begin{tabular}{l|l|l} 
Datum & Zeit & Ort \\
\hline $1670,6$. Juli & 1 Uhr & Neuchâtel
\end{tabular}

\section{Bewertung des Ereignisses}

Kein Erdbeben: Datierungsfehler.

\section{Korrektur}

1670, 17. Juli, Hall, A

\section{Überlieferung u. a. in:}

- Volger (1857)

- Kopp (1861)

- Hammerl \& Lenhardt (1997)

- MECOS-99

\section{Interpretation}

Die MECOS-99-Datierung auf den 6. Juli 1670 und die Schweizer Beobachtungen aus den protestantischen Gegenden mit der Datierung „7. Juli 1670“ müssen auf den „17. Juli“ im gregorianischen Kalender umgerechnet werden, dem Datum des grossen Schadenbebens von Hall im Tirol, das mit einer Intensität 8 bewertet wird (Hammerl \& Lenhardt, 1997). Ein Erdbeben, das auch noch in der Ostschweiz deutlich verspürt worden ist, so in St. Gallen, Salez (Rheintal) und wahrscheinlich auch in Zürich. Der Hinweis in der Kompilation von Kopp (1861) bezieht sich mit grosser Wahrscheinlichkeit nicht auf Neuenburg.

\section{Bemerkungen}

Vgl. das Erdbeben vom 17. Juli 1670 in Hall, A.

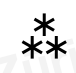

\begin{tabular}{l|l|l}
\hline Datum & Zeit & Ort \\
1670, 17. Juli & 1 Uhr 15 & Hall, Tirol, A
\end{tabular}

\section{Bewertung des Ereignisses}

Sicheres Ereignis.

\section{Parameter nach ECOS}

Ix: 8 
Überlieferung u. a. in:

- Volger (1857)

- Kopp (1861)

- Hammerl \& Lenhardt (1997)

- MECOS-99

\section{Quellen aus der Schweiz}

- StAZH: E II 35 a: Kirchlicher Brief (des Antistes?) aus Zürich an die Dekane, 21. (11.) Juli 1670

- StAZH: E II 35: Brief aus Salez vom 15. Mai 1671 von Jakob Zimmermann an den Antistes in Zürich

- $\quad$ StASG: Abt Gallus Alt.Diarium Illustrissimi Abbatis Galli II ab Anno 1665 usque 1672. tomus I, Stiftsarchiv St. Gallen, Bd. 265

\section{Interpretation}

Das grosse Schadenbeben von Hall im Tirol, A, wird mit einer Intensität 8 bewertet (Hammerl \& Lenhardt, 1997). Ein Erdbeben, das auch noch in der Ostschweiz deutlich verspürt worden ist, so in St. Gallen, Salez (Rheintal) und wahrscheinlich auch in Zürich. Der Hinweis in der Kompilation von Kopp (1861) bezieht sich mit grosser Wahrscheinlichkeit nicht auf Neuenburg. Die Schweizer Sitepoints und Hall, A, sind unten aufgeführt.

Mentalitätsgeschichtlich interessant ist ein Brief an die Dekane von Zürich zu diesem Erdbeben (StAZH E II 35 a), worin Erdbeben als ein Brüllen Gottes, als Ausdruck ernstlichen Zorns wegen schwerer Sünden der Menschen bezeichnet werden, was als Warnung zu verstehen sei.

Makroseismische Parameter (v. a. aus der Schweiz)

\begin{tabular}{|l|l|l|l|l|l|l|}
\hline Ortschaft & Länge & Breite & $I_{\min }$ & $I_{\max }$ & $I_{\text {w }}$ & Datenqualität \\
\hline Neuchâtel & 46.99 & 6.93 & & & verspürt & sehr schwach \\
\hline Kanton St. Gallen & 47.21 & 9.23 & & & verspürt & sehr schwach \\
\hline Schaffhausen & 47.7 & 8.64 & & & verspürt & sehr schwach \\
\hline Sax & 47.23 & 9.46 & & & verspürt & sehr schwach \\
\hline Hall, A & 47.28 & 11.52 & 8 & 8 & 8 & sehr schwach \\
\hline Zürich & 47.37 & 8.54 & 4 & 5 & 4 & sehr schwach \\
\hline Kanton Glarus & 46.98 & 9.06 & 4 & 5 & 4 & sehr schwach \\
\hline Salez & 47.24 & 9.5 & 4 & 5 & 4 & sehr schwach \\
\hline St. Gallen & 47.43 & 9.4 & 4 & 5 & 4 & sehr schwach \\
\hline Lindau am Bodensee & 47.56 & 9.7 & 3 & 5 & 4 & sehr schwach \\
\hline
\end{tabular}




\begin{tabular}{|l|l|l} 
Datum & Zeit & Ort \\
1670, 18. September & - & Glarus
\end{tabular}

\section{Bewertung des Ereignisses}

Kein Erdbeben: Datierungsfehler.

\section{Korrektur}

1670, 28. September

\section{Überlieferung}

Tschudi (1714) S. 613:

„Sonst haben sich in diesem Jahr (1670) abermals ein paar Erdbidem/als den 7. Julij (17. Juli, Hall, A) Morgens um 3 Uhr/und den 18. September Morgens um $10 \mathrm{Uhr} / \mathrm{mit}$ empfindlicher Erschütterung vermercken lassen.“

\section{Interpretation}

Der Kanton Glarus datiert noch bis $1701 \mathrm{im}$ julianischen Stil, sodass Tschudis Datierung um 10 Tage korrigiert werden muss.

\section{Bemerkungen}

Vgl. im ECOS-09 das Erdbeben vom 28. September 1670.

\section{**}

\begin{tabular}{|l|l|l} 
Datum & Zeit & Ort \\
1670, 2. Dezember & - & Zürich
\end{tabular}

\section{Bewertung des Ereignisses}

Kein Erdbeben: Datierungsfehler.

\section{Korrektur}

1670, 12. Dezember

\section{Überlieferung}

- Ziegler (1674)

- Volger (1857) 


\section{Interpretation}

Volger stützt sich auf die zeitgenössische Kompilation von Ziegler, der keine Quellenangaben gibt, sodass neben der Kalenderkorrektur dieser Hinweis als „sehr unsicher“ gewertet werden muss.

\section{Bemerkungen}

Vgl. das Erdbeben vom 12. Dezember 1670 im ECOS-09.

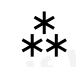

\begin{tabular}{l|l|l} 
Datum & Zeit (UTC) & Ort \\
$-1671,22$. Mai & - & - Sax, SG \\
$-1672,12$. Mai & -10 Uhr 30 & - Buchs, SG \\
$-1672,22$. Mai & -11 Uhr 30 & - Buchs, SG
\end{tabular}

\section{Bewertung des Ereignisses}

Kein Erdbeben: Datierungsfehler.

\section{Korrektur}

23. Mai, 1671 um 12 Uhr (UTC) in Sennwald, SG

\section{Überlieferung u. a. in:}

- Scheuchzer (1706/1716/1746)

- Bertrand (1756)

- Speicher (1815), StAAR

- Volger (1857)

Zeitgenössische Quelle:

Brief von Jacob Zimmermann aus Salez, 15. Mai 1671 (jul.) (StAZH: E II 35):

„Ehrwürdiger, hoch und wolgelehrter Hr. Antistes wie der allmächtige Gott seinen starken arm den 7. Juli verwichenes Jahrs ${ }^{28}$ mit empfündtlicher bewegung des erd bodens uns nebet anderen merklichen hat spüren lassen; desto hat der selbige verwichen sambstag .... ${ }^{29}$ miten tag uns aber mahlen denselbigen mit starker Bewegung de erdtich uns empfinden lassen, nach miten tag ep zwüschet 1. und 2. uhren weliches sonderbahr vermerkte worden in beiden meinen Gemeinden zu Saletz ${ }^{30}$ und in Hag ${ }^{31}$ wi auch zu Frumbsen ${ }^{32}$ und zu Sax mit gewaltigem erschütern und zitren, gott wölle dass unser aller hertzen ...durch zu mehrer und ...gleichs... buss bewegt werdind!“

\footnotetext{
28 7. Juli 1670 / 17. Juli 1670 , Hall im Tirol, Io: 8.

29 13. Mai 1671, jul., Samstag / 23. Mai 1671 greg.

30 Salez

31 Haag

32 Frümsen
} 


\section{Interpretation}

Es liegt nur eine einzige zeitgenössische Beobachtung vor, auf die sich der Kompilator Scheuchzer und dessen Kopisten mit grosser Wahrscheinlichkeit teilweise stützen. Scheuchzer zeigt dieselbe Lokalisierung wie der Brief, weicht jedoch in der Jahres- und Tagesbezeichnung ab. Mangels weiterer Quellen wird deshalb vorläufig die Datierung aus Zimmermanns Brief, also Samstag vor dem 15. Mai 1671 (Montag, 13. Mai, jul.) beziehungsweise 23. Mai (greg.) übernommen. Sax liegt im heutigen Sennwald, SG.

Erst seit Bertrand (1756) wird von Schäden am 22. (12.) Mai gesprochen, die jedoch nicht zeitgenössisch belegt werden können.

\section{Bemerkungen}

Vgl. im ECOS-09 das Erdbeben vom 23. Mai 1671 in Sennwald, SG.

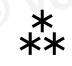

Datum

- 1672, 9. Januar

- 1672, 12. Januar
Zeit (UTC)

- 14 Uhr

- 10 Uhr 30
Ort

- Hohensax, in Sennwald, SG

- Sax, in Sennwald, SG

\section{Bewertung des Ereignisses}

Keine Erdbeben: Datierungsfehler.

\section{Korrektur}

1672, 19. Januar, $14 \mathrm{Uhr}$

\section{Überlieferung u. a. in:}

- Scheuchzer (1706)

- Memorabilia Tigurina (1710)

- Bertrand (1756)

- Volger (1857)

- Senn (1860)

- Gils, Van \& Leydecker (1991)

- MECOS-99

\section{Quellen}

- StAZH A 346.4: Hofmeister Salomon

- StAZH E II 35: Zimmermann, Brief vom 15. Mai 1671 (jul.) 


\section{Interpretation}

Der älteste Hinweis auf das Beben vom 9. Januar stammt aus Scheuchzer:

„A. 1672. den 9. Jan. Nachmittag um 3. Uhr ist in der Herrschaft Sax ein Erdbeben verspürt worden mit einer zimlichen Erschütterung und Geräusche; zu Frümsen aber mit einem lauten Klapf und Getöse. (Ex Archivo Antistit. Tigurin.)“

Er muss auf den 19. Januar 1672 umdatiert werden, da er aus den Zürcher Herrschaften stammt.

\section{Bemerkungen}

Vgl. das Erdbeben vom 19. Januar im ECOS-09.

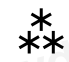

\begin{tabular}{l|l|l} 
Datum & Zeit & Ort \\
$1672,2$. Dezember & $14 \mathrm{Uhr}$ & Kanton Zürich
\end{tabular}

\section{Bewertung des Ereignisses}

Kein Erdbeben: Datierungsfehler.

\section{Korrektur}

1672, 12. Dezember

\section{Überlieferung u. a. in:}

- Scheuchzer (1706)

- Bertrand (1756)

- $\quad$ olger (1857)

- MECOS-99

Zeitgenössische Quelle:

StiAEi: Dietrich Josef, Einsiedeln, Sign.: A HB 1, 12. Decembris 1672.

\section{Interpretation}

Dieses julianische Datum stammt z. B. aus den Kompilationen von Scheuchzer, Bertrand und Volger und ist auf den 12. Dezember, gregorianischer Kalender, zu verschieben, wie die zeitgenössische Beobachtung von Josef Dietrich aus dem katholischen Einsiedeln belegt: „Terraemotus, 12. Decembris ist ohngefehr umb 3. Vierthel vor 3 Uhr vesperzeiths ein zimlich starkhes Erdbeben vermerkhet worden. Wo selbiges ausgebrochen, und was dardurch für Schaden möchte erfolget sein, stehet zu erwarten.“

Der Kompilator Scheuchzer (1706) zählt Zürcher Beobachtungen auf (S. 128): „Anno 1672. den 2. Dec. Anf den abend gegen 3 Uhren ist zu Uster/Eglisau/Kyburg/und andern Ohrten ein gar empfindlicher Erdbidem vermerkt worden. Auf dem Thurn zu Eglisau gewahrte der Wächter erstlich ein kleines geräusch/und darbey die Geschirr auf den 
Gestellen sich bewegen/und als er darauf zum Fenster hinauss gesehen/den Thurn/die Fenster/und sich selbst hin und har bewegen. (Ex Archiv. Antistit. Tigurin.)“

Es werden keine Schäden aufgeführt.

\section{Bemerkungen}

Vgl. das Erdbeben vom 12. Dezember 1672 im ECOS-09.

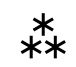

\begin{tabular}{l|l|l} 
Datum & Zeit (UTC) & Ort \\
$-\quad 1674,6$. November & $-8 \mathrm{Uhr}$ & $-(-)$ \\
$-\quad 1674,6$. Dezember & $-8 \mathrm{Uhr}$ & - Glarus
\end{tabular}

\section{Bewertung des Ereignisses}

Keine Erdbeben: Datierungsfehler.

\section{Korrektur}

1674, 16. Dezember in Thalwil, ZH

\section{Überlieferung u. a. in:}

- Lindiner (1711)

- Philipert, J. H.: UBB, Handschriften, VB Mscr.

- Isaak Vetter, o. J., Archiv Stein am Rhein, 1. Hälfte 18. Jh., stützt sich auf „Conr. Sul. Mscrpt."

- Perrey (1845)

- Naef (1850)

- Candreia (1905)

- Giessberger (1922)

- $\quad$ Sieberg (1940)

- MECOS-99

- SisFrance (2008)

Zeitgenössische Beobachtungen:

- Ziegler (1674)

- StAZH, Pfarrbuch Kilchberg: E III 062.2

- Sauter Chronik, LAAI

- Haltmeyer (1683), Vadiana: S 1592 b

\section{Interpretation}

Bei diesem Ereignis sind in den Kompilationen zwei Datierungsfehler aufgetreten, einerseits ist die Bezeichnung „Wintermonat“ mit November übersetzt worden, was es zwar 
meist auch bedeutet, es kann aber auch der Dezember gemeint sein, andererseits handelt es sich beim 6. Dezember um eine julianische Datierung, wie die zeitgenössischen Quellen belegen, sodass auf den 16. Dezember 1674 umdatiert werden muss.

\section{Bemerkung}

Vgl. das Erdbeben vom 16. Dezember 1674.

\begin{tabular}{|c|c|c|}
\hline $\begin{array}{l}\text { Datum } \\
\text { 1674, 16. Dezember }\end{array}$ & $\begin{array}{l}\text { Zeit (UTC) } \\
8 \mathrm{Uhr}\end{array}$ & $\begin{array}{l}\text { Ort } \\
\text { Thalwil, ZH }\end{array}$ \\
\hline
\end{tabular}

\section{Bewertung des Ereignisses}

Sicheres Ereignis.

\section{Parameter nach ECOS}

Ix: 5

\section{Überlieferung u. a. in:}

- Lindiner (1711)

- Philipert, J. H.: UBB, Handschriften, VB Mscr. 07

- Isaak Vetter, o. J., Archiv Stein am Rhein, 1. Hälfte 18. Jh., stützt sich auf „Conr. Sul. Mscrpt.“

- Perrey (1845)

- $\quad$ Naef (1850)

- Candreia (1905)

- Giessberger (1922)

- Sieberg (1940)

- MECOS-99

- SisFrance (2008)

- Strasser (2008)

\section{Quellen}

- Ziegler (1674)

- StAZH, Pfarrbuch Kilchberg: E III 062.2

- Sauter Chronik, LAAI, Sign.: 60, 61

- Haltmeyer (1683), Vadiana: S 1592 b

\section{Interpretation}

Bei diesem Ereignis sind in den Kompilationen zwei Datierungsfehler aufgetreten, einerseits ist die Bezeichnung „Wintermonat“ mit November übersetzt worden, was es zwar 
meist auch bedeutet, es kann aber auch der Dezember gemeint sein, andererseits handelt es sich beim 6. Dezember um eine julianische Datierung, wie die zeitgenössischen Quellen belegen, sodass auf den 16. Dezember 1674 umdatiert werden muss.

Das Kilchberger Pfarrbuch umschreibt das Ereignis vom Dezember 1674 folgendermassen:

„Sontags den 6. dess Morgens umb 8 1/2 Uhren ehe man zur Predig gienge war bey starkhem Nebel wideren (?) ein empfindtlicher Erdbidem gspührt, ginge fast durch die gantze Eidtgnoschafft und benachbarte lant. Gott wende boser bedäutung ab, (...).“

Und Ziegler hält folgendes fest: „Anno 1674. Sontags den 6. Christm. (Dezember) morgens um 9 uhr / ward ein starker Erdbidem / durch die gantze Eidgnossschaft / und andere angräntzende Länder verspüret / alss man vast aller orten den Gottesdienst verrichtete. In der Statt Müllhausen / im Elsass / welches ein mit verbündet und zugewandt Ort der Eidgnossschaft ist / solle dieses Erdbeben / die Kirchenthürn erschüttet haben / dass die Gloggen einen Thon von sich gegeben / als wann sie angezogen wären / zum Leuten." Da Ziegler und Haltmeyer die Zeit auf 9 Uhr festlegen, wird das Erdbeben entsprechend auf 8 Uhr UTC fixiert.

Erdbebenschäden werden keine erwähnt, die Intensität wird aber mit „empfindlich“, „stark“, „heftig“ und „entsetzlich“ umschrieben. In St. Gallen sei, gemäss dem Kompilatoren Naef (1850), bei der Flucht aus der Kirche eine Frau im Gedränge erdrückt worden. Da dieser Unfall nicht zeitgenössisch belegt werden kann - er fehlt in der St. Galler Chronik von Haltmeyer - und eher auf Panik beruht, kann nicht auf ein Schadenbeben geschlossen werden. Die zu hohe Bewertung dieses Erdbebens durch Strasser (2008) beruht auf der irrtümlich höheren Einschätzung im ECOS-02 (5.4 statt 4.5).

\section{Makroseismische Parameter}

\begin{tabular}{|l|l|l|l|l|l|l|}
\hline Ortschaft & Länge & Breite & $I_{\min }$ & $I_{\max }$ & $I_{\text {w }}$ & Datenqualität \\
\hline Appenzell & 47.33 & 9.41 & 4 & 5 & 5 & sehr schwach \\
\hline Basel & 47.56 & 7.59 & 4 & 5 & 5 & sehr schwach \\
\hline Zürich & 47.37 & 8.54 & 4 & 5 & 5 & sehr schwach \\
\hline Mulhouse & 47.75 & 7.34 & 4 & 5 & 5 & sehr schwach \\
\hline Knonau & 47.22 & 8.46 & 4 & 4 & 5 & sehr schwach \\
\hline St. Gallen & 47.43 & 9.4 & 4 & 5 & 5 & sehr schwach \\
\hline Kilchberg ZH & 47.32 & 8.54 & 4 & 5 & 4 & sehr schwach \\
\hline
\end{tabular}




\begin{tabular}{|l|l|l}
\hline Datum & Zeit & Ort \\
\hline $1676,17$. Juni & $20 \mathrm{Uhr}$ & Ivrea, I
\end{tabular}

\section{Bewertung des Ereignisses}

Kein Ereignis.

\section{Korrektur}

\section{Überlieferung}

- Baratta (1905)

- Rüttener (1995)

- MECOS-99

Nicht vorhanden in folgenden Schweizer Kompilationen:

- Volger (1857)

- Candreia (1905)

- Montandon (1942-43)

Abgelehnt:

- Boschi et al. (1997)

\section{Interpretation}

Es wurden keine Schweizer Quellen zu diesem angeblichen Ereignis im Piemont gefunden, sodass aufgrund der Arbeit von Boschi (1997) auf eine Falschmeldung geschlossen wird.

\section{***}

\begin{tabular}{|c|c|c|}
\hline $\begin{array}{l}\text { Datum } \\
\text { 1677, 13. Dezember }\end{array}$ & $\begin{array}{l}\text { Zeit (UTC) } \\
6 \text { Uhr }\end{array}$ & $\begin{array}{l}\text { Ort } \\
\text { - } \quad \text { St. Gallen } \\
\text { - } \quad \text { Gais, AR }\end{array}$ \\
\hline
\end{tabular}

\section{Bewertung des Ereignisses}

Kein Erdbeben: Datierungsfehler.

\section{Korrektur}

1677, 23. Dezember in St. Gallen

Überlieferung u. a. in:

- Scheuchzer (1706)

- Isaak Vetter (1692-1742), Manuskript StASaR: Bü 49 
- Volger (1857)

- MECOS-99

Zeitgenössische Beobachtungen:

- Josef Dietrich, Einsiedeln Dietrich Joseph, StiAEi: A HB 1-11, Pfäffikon: B Tg 2

- StAZH: E III 053.1. Pfarreibuch Hirzel, 1617-1754: 1677

- Haltmeyer (1683)

\section{Interpretation}

Die Datierung 13. Dezember stammt aus Quellen protestantischer Gegenden mit julianischem Kalender, während im gregorianisch datierten Tagebuch von Josef Dietrich aus dem Kloster Einsiedeln der 23. Dezember genannt wird. Schäden werden keine erwähnt, die Intensität wird mit „verspürt“ (Zürich), „nicht gar stark“ (Einsiedeln), „stark“ (St. Gallen) und „ziemlich stark“ (Zürich) umschrieben.

\section{Bemerkung}

Vgl. im ECOS-09 das Erdbeben vom 23. Dezember 1677.

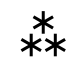

Datum

1679, 2. Januar
Zeit (UTC)

2 Uhr 30
Ort

St. Gallen

\section{Bewertung des Ereignisses}

Kein Erdbeben: Datierungsfehler.

\section{Korrektur}

1679, 12. Januar

\section{Überlieferung}

Haltmeyer (1683)

\section{Interpretation}

Haltmeyer, S. 669: „Desselbigen Jahrs (1679) den 2. Tag Jenner/morgens zwischen 2. und 3. Uhren ist widerum ein Erdbidem gewesen."

Im Gegensatz zum Kloster St. Gallen wechselte die Stadt St. Gallen den Kalenderstil erst 1701, sodass es sich hier mit grosser Wahrscheinlichkeit um eine julianische Datierung handeln muss, die um zehn Tage zu korrigieren ist; bestätigende zeitgenössische Berichte fehlen allerdings noch. 


\section{Bemerkung}

Vgl. das Erdbeben vom 12. Januar 1679.

\begin{tabular}{l|l|l|l|l|l|l|} 
& $* * *$ \\
& & \\
& & \\
Datum & Zeit & Ort \\
1680, 24. Juni & - & Schweiz
\end{tabular}

\section{Bewertung des Ereignisses}

Kein Erdbeben: Datierungsfehler.

\section{Korrektur}

1680, 4. Juli

\section{Überlieferung}

- Trümpi (1774)

- Volger (1857)

\section{Interpretation}

Volger (1857) zählt Hinweise aus zahlreichen Kompilationen auf, die aus protestantischen Kantonen stammen und damit um 10 Tage zu korrigieren sind. Da auch noch Ungewitter erwähnt werden und zeitgenössische Quellen fehlen, ist das Ereignis an sich als sehr unsicher zu werten. Falls es sich noch um eine Juni-Juli-Verwechslung handeln sollte, so könnte der 3. August gemeint sein, wofür mit Josef Dietrich aus Einsiedeln eine zuverlässige zeitgenössische Quelle vorhanden ist.

\section{Bemerkung}

Vgl. die Erdbeben vom 4. Juli und 3. August 1680.

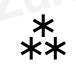

\begin{tabular}{|l|l|l}
\hline Datum & Zeit & Ort \\
\hline $1680,24$. Juli & - & St. Aubin, FR
\end{tabular}

\section{Bewertung des Ereignisses}

Kein Erdbeben: Datierungsfehler

\section{Korrektur}

1680, 3. August 


\section{Überlieferung}

- Lindiner, 1711

- Tschudi, 1714

- Dettling, A. 1900-21

Zeitgenössische Quellen:

- Joseph Dietrich (StiAEi: A HB 1-11)

\section{Interpretation}

Der zeitgenössische Hinweis aus Einsiedeln von Joseph Dietrich belegt, dass gregorianisch auf den 3. August 1680 umdatiert werden muss. Es werden keine Schäden erwähnt.

\section{Bemerkung}

Vgl. die Erdbeben vom 3. August 1680 in Zürich. 


\section{Abkürzungsverzeichnis}

\subsection{Kantonskürzel und Länderkürzel}

\begin{tabular}{|c|c|}
\hline AG & Aargau \\
\hline AR & Appenzell Ausserrhoden \\
\hline Al & Appenzell Innerrhoden \\
\hline $\mathrm{BL}$ & Basel Landschaft \\
\hline BS & Basel Stadt \\
\hline $\mathrm{BE}$ & Bern \\
\hline FR & Freiburg/Fribourg \\
\hline GE & Genève \\
\hline $\mathrm{GL}$ & Glarus \\
\hline GR & Graubünden \\
\hline JU & Jura \\
\hline LU & Luzern \\
\hline NE & Neuchâtel \\
\hline NW & Nidwalden \\
\hline OW & Obwalden \\
\hline $\mathrm{SH}$ & Schaffhausen \\
\hline SZ & Schwyz \\
\hline SG & St. Gallen \\
\hline SO & Solothurn \\
\hline TI & Ticino \\
\hline TG & Thurgau \\
\hline UR & Uri \\
\hline VD & Vaud \\
\hline VS & Wallis/Valais \\
\hline$Z G$ & Zug \\
\hline $\mathrm{ZH}$ & Zürich \\
\hline A & Österreich \\
\hline D & Deutschland \\
\hline$F$ & Frankreich \\
\hline I & Italien \\
\hline$P$ & Portugal \\
\hline
\end{tabular}




\subsection{Weitere Abkürzungen}

Ix Maximal beobachtete Intensität (diese könnte auch überschritten worden sein)

Imin Minimale Intensität an einem Punkt (in der Regel in einer Ortschaft)

IMax Maximale Intensität an einem Punkt (in der Regel in einer Ortschaft)

Iw Wahrscheinliche Intensität an einem Punkt (in der Regel in einer Ortschaft)

UTC Universal Time Coordinated (Koordinierte Weltzeit) 


\section{Tabellen}

\subsection{Tabelle aller Erdbeben der Schweiz mit $I_{x} \geq 6$ von $1000-1680$}

\begin{tabular}{|c|c|c|c|c|c|c|}
\hline Jahr & Mt & Tag & Std & Min & Intensität & Lokalisierung \\
\hline 1117 & 1 & 3 & 13 & & 9.5 & Veronese, I \\
\hline 1295 & 9 & 3 & & & 8 & Churwalden, GR \\
\hline 1348 & 1 & 25 & & & 9.5 & Friuli, I (früher Villach, A) \\
\hline 1356 & 10 & 18 & 16 & & 7 & Basel \\
\hline 1356 & 10 & 18 & 21 & & 9 & Basel \\
\hline 1357 & 5 & 8 & 16 & & 7 & Strasbourg, F \\
\hline 1416 & 7 & 21 & 14 & & 6 & nicht lokalisierbar (Basel) \\
\hline 1428 & 12 & 12 & & & 7 & nicht lokalisierbar (Basel) \\
\hline 1498 & 9 & 3 & 14 & & 6 & nicht lokalisierbar (Saanen, BE) \\
\hline 1504 & 3 & & & & 7 & Ardez, GR \\
\hline 1523 & 12 & 27 & 23 & & 6.5 & Freiburg i. Br., D \\
\hline 1524 & 4 & & & & 8 & Ardon, VS \\
\hline 1569 & 8 & 6 & $4 ?$ & & 6 & nicht lokalisierbar (Basel) \\
\hline 1574 & & & & & - & Offenburg, D \\
\hline 1584 & 3 & 11 & 11 & 30 & 8 & Aigle, VD \\
\hline 1584 & 3 & 14 & 8 & & 7 & Aigle, VD \\
\hline 1588 & 6 & 21 & & & 6 & nicht lokalisierbar (Singen, D) \\
\hline 1601 & 9 & 18 & 1 & & 8 & Unterwalden \\
\hline 1610 & 12 & 9 & & & 7 & nicht lokalisierbar (Basel) \\
\hline 1622 & 8 & 3 & & & 7 & Ftan, GR \\
\hline 1642 & 6 & 13 & 22 & & 6.5 & Bergamo, I \\
\hline 1650 & 9 & 21 & 3 & & 7 & Basel \\
\hline 1655 & 3 & & & & - & Tübingen, D \\
\hline 1655 & 4 & & & & - & Tübingen, D \\
\hline 1655 & & & & & 6 & nicht lokalisierbar (Lausanne) \\
\hline 1660 & & & & & 6 & nicht lokalisierbar (Lausanne) \\
\hline 1665 & & & & & 6 & nicht lokalisierbar (Lausanne) \\
\hline 1669 & 10 & 10 & 12 & 45 & 6 & Plaine de Basse - Alsace, $F$ \\
\hline 1670 & 7 & 17 & 1 & 15 & 8 & Hall in Tirol, $A$ \\
\hline
\end{tabular}




\subsection{Tabelle ausgewählter schwacher Erdbeben 1000-1680}

\begin{tabular}{|c|c|c|c|c|c|c|}
\hline Jahr & Mt & Tag & Std & Min & Intensität & Lokalisierung \\
\hline 1021 & 5 & 12 & & & verspürt & nicht lokalisierbar (Bayern-Ostschweiz) \\
\hline 1046 & 11 & 9 & & & verspürt & nicht lokalisierbar (Süddtld. - I) \\
\hline 1048 & 10 & 13 & & & 5 & Bayern-Bodensee \\
\hline 1062 & 2 & 8 & & & verspürt & nicht lokalisierbar (Reichenau, D) \\
\hline 1092 & 12 & & & & 3 & nicht lokalisierbar (Schaffhausen) \\
\hline 1117 & 1 & 3 & 1 & & 5 & Süddeutschland \\
\hline 1127 & 3 & 24 & & & verspürt & nicht lokalisierbar (St. Blasien) \\
\hline 1127 & 4 & 13 & & & verspürt & nicht lokalisierbar (Regensburg) \\
\hline 1128 & 6 & 29 & & & verspürt & $\begin{array}{l}\text { nicht lokalisierbar (St. Blasien - Ell- } \\
\text { wangen, D) }\end{array}$ \\
\hline 1134 & 1 & 12 & & & verspürt & nicht lokalisierbar (St. Blasien, D) \\
\hline 1155 & 1 & 18 & & & verspürt & Laon, F? \\
\hline 1158 & 1 & 21 & $23(?)$ & & verspürt & nicht lokalisierbar (Engelberg) \\
\hline 1161 & 2 & 22 & $16(?)$ & & verspürt & nicht lokalisierbar (Engelberg) \\
\hline 1161 & 7 & 16 & 16 & & verspürt & nicht lokalisierbar (Engelberg) \\
\hline 1162 & 2 & 25 & 23 & & verspürt & nicht lokalisierbar (Engelberg) \\
\hline 1162 & 12 & 2 & 1 & & verspürt & nicht lokalisierbar (Engelberg) \\
\hline 1170 & 4 & 1 & $17(?)$ & & verspürt & nicht lokalisierbar (Engelberg) \\
\hline 1170 & 4 & 1 & $23(?)$ & & verspürt & nicht lokalisierbar (Engelberg) \\
\hline 1175 & 4 & 30 & $19(?)$ & & verspürt & nicht lokalisierbar (Engelberg) \\
\hline 1201 & 5 & 4 & 10 & & keine Wertung & Katschberg (Kärnten), A \\
\hline 1215 & 5 & 21 & 1 & & verspürt & nicht lokalisierbar (Nordostschweiz) \\
\hline 1258 & 2 & 18 & 17 & & verspürt & nicht lokalisierbar (Chur) \\
\hline 1277 & 6 & 9 & & & verspürt & nicht lokalisierbar (Konstanz, D) \\
\hline 1280 & 10 & 26 & & & verspürt & nicht lokalisierbar (Colmar, F) \\
\hline 1295 & 4 & 3 & & & 5 & nicht lokalisierbar (Colmar, F) \\
\hline 1305 & 10 & 21 & & & keine Wertung & Como, I \\
\hline 1322 & 10 & 16 & & & verspürt & $\begin{array}{l}\text { nicht lokalisierbar (Lausanne - Genè- } \\
\text { ve) }\end{array}$ \\
\hline 1363 & 6 & 24 & & & verspürt & nicht lokalisierbar (Strasbourg, F) \\
\hline 1372 & 6 & 1 & & & verspürt & nicht lokalisierbar (Strasbourg, F) \\
\hline
\end{tabular}




\begin{tabular}{|c|c|c|c|c|c|c|}
\hline Jahr & Mt & Tag & Std & Min & Intensität & Lokalisierung \\
\hline 1384 & 12 & 25 & & & verspürt & $\begin{array}{l}\text { nicht lokalisierbar (Augsburg-Nörd- } \\
\text { lingen, D) }\end{array}$ \\
\hline 1394 & 4 & 22 & & & 5 & nicht lokalisierbar (Zürich) \\
\hline 1402 & 4 & 14 & & & verspürt & nicht lokalisierbar (Basel) \\
\hline 1403 & 11 & 23 & & & verspürt & nicht lokalisierbar (Basel) \\
\hline 1498 & 4 & & 9 & & 5 & nicht lokalisierbar (Saanen, BE) \\
\hline 1506 & 9 & 7 & 8 & & verspürt & nicht lokalisierbar (Basel) \\
\hline 1506 & 10 & 4 & 23 & & verspürt & nicht lokalisierbar (Basel) \\
\hline 1531 & 10 & 10 & 20 & & 5 & St. Gallen \\
\hline 1533 & 11 & 17 & & & 5 & St. Gallen \\
\hline 1541 & 1 & 6 & & & verspürt & nicht lokalisierbar (Urseren, UR) \\
\hline 1542 & 11 & 8 & 8 & & keine Wertung & Leutkirch, D \\
\hline 1571 & 2 & 19 & 7 & & 4 & Basel \\
\hline 1573 & 5 & 27 & 18 & & 4 & nicht lokalisierbar (Chur) \\
\hline 1573 & 6 & 4 & & & 5 & nicht lokalisierbar (Chur) \\
\hline 1573 & 6 & 5 & & & verspürt & nicht lokalisierbar (Chur) \\
\hline 1573 & 6 & 6 & & & 5 & nicht lokalisierbar (Chur) \\
\hline 1573 & 7 & 8 & 19 & & verspürt & nicht lokalisierbar (Chur) \\
\hline 1573 & 12 & 21 & & & 5 & Stein am Rhein, SH \\
\hline 1574 & 7 & 30 & & & 4 & Zürich \\
\hline 1577 & 9 & 22 & & & 5 & nicht lokalisierbar (Bern - Colmar) \\
\hline 1616 & 2 & 29 & 4 & & 5 & nicht lokalisierbar (Zürich) \\
\hline 1621 & 5 & 30 & & & 5 & Neuchâtel \\
\hline 1621 & 5 & 31 & & & verspürt & Neuchâtel \\
\hline 1635 & 6 & 20 & 21 & & verspürt & nicht lokalisierbar (Zürich) \\
\hline 1648 & 12 & 3 & & & verspürt & nicht lokalisierbar (Neuchâtel) \\
\hline 1652 & 2 & 11 & & & verspürt & nicht lokalisierbar (Nordschweiz) \\
\hline 1652 & 10 & 23 & 11 & & verspürt & nicht lokalisierbar (Rapperswil, SG) \\
\hline 1674 & 12 & 16 & 8 & & 5 & Thalwil, ZH \\
\hline
\end{tabular}




\subsection{Tabelle der Falschmeldungen 1000-1680}

\begin{tabular}{|c|c|c|c|c|c|}
\hline Jahr & Mt & Tag & Std & Min & Lokalisierung \\
\hline 1000 & 3 & 29 & & & Schweiz \\
\hline 1001 & & & & & Schweiz \\
\hline 1020 & 5 & 12 & & & Bayern (Würzburg), D \\
\hline 1021 & 5 & 12 & & & Basel \\
\hline 1048 & 10 & 15 & & & Konstanz, D \\
\hline 1048 & 10 & 16 & & & Konstanz, D \\
\hline 1051 & & & & & Breisgau, D \\
\hline 1081 & 3 & 27 & & & Schweiz \\
\hline 1092 & 2 & 8 & & & Konstanz, D \\
\hline 1098 & & & & & Reinach-Basel \\
\hline 1107 & & & & & Süddeutschland \\
\hline 1112 & 1 & 3 & & & Rottenburg-Neckar, D \\
\hline 1115 & & & & & Zürich \\
\hline 1127 & 3 & 25 & 23 & & Waldshut-Tiengen, D \\
\hline 1128 & & & & & Reinach-Basel \\
\hline 1134 & 1 & 12 & & & Waldshut-Tiengen, D \\
\hline 1152 & & & & & Neuchâtel \\
\hline 1155 & 1 & 18 & 17 & & Waldshut-Tiengen, D \\
\hline 1161 & 1 & 16 & & & Konstanz, D \\
\hline 1170 & & & & & Lugano \\
\hline 1170 & 4 & 1 & 17 u. 23 & & St. Blasien, D \\
\hline 1170 & 6 & 29 & & & Bad Säckingen, D, Steiermark, A \\
\hline 1180 & 8 & 1 & 3 & & Stans \\
\hline 1201 & 5 & 4 & 15 & & Bayern, D, Steiermark, A \\
\hline 1239 & 9 & & & & Colmar \\
\hline 1265 & 6 & 19 & & & St. Blasien, D \\
\hline 1277 & 5 & 27 & & & Konstanz \\
\hline 1279 & 9 & 2 & & & Strasbourg, F \\
\hline 1290 & & & & & Schweiz/Innertkirchen, BE \\
\hline 1295 & 4 & 5 & & & Chur und Reichenau, GR \\
\hline 1295 & 9 & 4 & & & Chur \\
\hline
\end{tabular}




\begin{tabular}{|c|c|c|c|c|c|}
\hline Jahr & Mt & Tag & Std & Min & Lokalisierung \\
\hline 1301 & 9 & 4 & & & Lindau \\
\hline 1322 & 10 & 31 & & & Genève \\
\hline 1322 & 11 & & & & Genève \\
\hline 1334 & 12 & 4 & 23 & & Monte Baldo - Lago di Garda, I \\
\hline 1336 & 10 & 8 & & & Basel \\
\hline 1346 & 11 & 24 & & & Basel \\
\hline 1350 & & & & & Adelboden \\
\hline 1357 & 5 & 9 & & & Strasbourg \\
\hline 1357 & 5 & 14 & & & Strasbourg \\
\hline 1358 & & & & & Chur \\
\hline 1363 & 7 & 3 & & & Thann, F \\
\hline 1364 & 5 & 11 & & & Thann - Haut Rhin, F \\
\hline 1369 & 11 & 26 & & & Milano \\
\hline 1372 & 6 & 1 & & & Aesch - Basel \\
\hline 1372 & 9 & 8 & & & Aesch - Basel \\
\hline 1375 & & & & & Beckenried, NW \\
\hline 1378 & 6 & 1 & & & Glarus und Klönthal, GL \\
\hline 1380 & 7 & 1 & & & Bern \\
\hline 1382 & 4 & 20 & & & Col de Balme - Chamonix, F \\
\hline 1384 & 12 & 4 & & & Thann - Haut Rhin, F \\
\hline 1391 & 3 & 22 & & & Sundgau, F - Schweiz \\
\hline 1394 & 3 & 22 & & & Brig, vS \\
\hline 1396 & 12 & 26 & & & Bergamo, I \\
\hline 1397 & 12 & 26 & 2 & & Bergamo, I \\
\hline 1400 & & & & & Kreuzlingen, TG - Bodenseegebiet \\
\hline 1415 & 6 & 21 & & & Basel \\
\hline 1416 & 7 & 22 & & & Basel \\
\hline 1417 & & & & & Neuchâtel \\
\hline 1428 & 12 & 13 & & & Basel \\
\hline 1470 & 2 & 6 & 4 & & Aesch - Basel \\
\hline 1471 & 5 & & & & Nördlingen, D \\
\hline 1474 & 12 & 11 & 14 & & Basel \\
\hline 1490 & & & & & Lugano \\
\hline
\end{tabular}




\begin{tabular}{|c|c|c|c|c|c|}
\hline Jahr & Mt & Tag & Std & Min & Lokalisierung \\
\hline 1492 & 11 & 7 & & & Aesch - Basel \\
\hline 1498 & 3 & 31 & & & Zweisimmen \\
\hline 1500 & & & & & Neuchâtel \\
\hline 1500 & 4 & 30 & & & Einsiedeln, SZ \\
\hline 1500 & 5 & & & & Schweiz \\
\hline 1506 & 9 & 22 & & & Basel \\
\hline 1507 & 9 & 7 & 9 & & Basel \\
\hline 1508 & & & & & Albula, GR \\
\hline 1512 & & & & & Biasca \\
\hline 1513 & 2 & 10 & & & Bellinzona \\
\hline 1513 & 9 & 30 & & & Biasca - Val Blenio \\
\hline 1517 & 4 & 4 & & & Böblingen, D \\
\hline 1517 & 6 & 26 & & & Nördlingen, D \\
\hline 1523 & 5 & 19 & 2 & & Suisse romande \\
\hline 1524 & 12 & 27 & & & Basel \\
\hline 1531 & 1 & 26 & & & Schweiz \\
\hline 1533 & 10 & 22 & & & Zürich \\
\hline 1533 & 11 & 17 & & & Chur \\
\hline 1533 & 11 & 26 & 0 & 30 & St. Margrethen, SG \\
\hline 1534 & 10 & 2 & & & Baden \\
\hline 1537 & 3 & 1 & & & Basel \\
\hline 1538 & 1 & 28 & & & Basel \\
\hline 1552 & 2 & 9 & & & Mulhouse, F \\
\hline 1555 & 11 & 2 & & & Sondrio, I \\
\hline 1559 & & & & & Chur \\
\hline 1569 & 4 & 6 & 8 & & Bern \\
\hline 1572 & 2 & 9 & 7 & & Basel \\
\hline 1572 & 2 & 19 & & & Breisgau, D \\
\hline 1572 & 6 & 1 & & & Basel \\
\hline 1573 & 6 & 30 & & & Chur \\
\hline 1573 & 12 & 20 & & & Klöntal, GL \\
\hline 1573 & 12 & 21 & & & Glarus \\
\hline 1574 & 5 & 3 & & & Genève \\
\hline
\end{tabular}




\begin{tabular}{|c|c|c|c|c|c|}
\hline Jahr & Mt & Tag & Std & Min & Lokalisierung \\
\hline 1574 & 6 & 30 & & & Zürich \\
\hline 1584 & 3 & 1 & 11 & & Aigle \\
\hline 1584 & 3 & 1 & & & Dronero, I \\
\hline 1584 & 3 & 2 & & & Aigle \\
\hline 1584 & 3 & 3 & & & Aigle \\
\hline 1584 & 3 & 4 & & & Aigle \\
\hline 1584 & 3 & 10 & & & Aigle \\
\hline 1584 & 5 & 4 & & & Aigle \\
\hline 1585 & 5 & 4 & & & Basel \\
\hline 1588 & 6 & 11 & & & Singen, D \\
\hline 1593 & 2 & 6 & & & Nördlingen, D \\
\hline 1593 & 10 & 10 & & & Klöntal, GL \\
\hline 1593 & 11 & 5 & & & Neuchâtel \\
\hline 1593 & 11 & 10 & & & Glarus \\
\hline 1593 & 11 & 11 & 17 & & Glarus \\
\hline 1593 & 11 & 21 & & & Glarus \\
\hline 1594 & 3 & & & & Glarus \\
\hline 1594 & 11 & 11 & & & Glarus \\
\hline 1597 & 9 & 10 & & & Simplon, VS \\
\hline 1599 & 10 & 23 & & & Reichenau, GR \\
\hline 1600 & 9 & 8 & & & Genève \\
\hline 1601 & 9 & 8 & 1 & & Engelberg \\
\hline 1602 & 6 & 28 & 5 & & Zürich \\
\hline 1605 & 11 & 20 & 3 & & Landquart, GR \\
\hline 1610 & 11 & 29 & & & Basel \\
\hline 1612 & 11 & 9 & & & Neuchâtel \\
\hline 1614 & 2 & 17 & & & Basel \\
\hline 1614 & 2 & 28 & & & Basel \\
\hline 1614 & 9 & 24 & 0 & 15 & Aesch-Basel \\
\hline 1616 & 2 & 19 & 4 & 30 & Zürich \\
\hline 1616 & 3 & 20 & & & Engelberg, OW \\
\hline 1618 & 8 & 25 & & & Chiavenna, I \\
\hline 1618 & 9 & 4 & & & Piuro, I \\
\hline
\end{tabular}




\begin{tabular}{|c|c|c|c|c|c|}
\hline Jahr & Mt & Tag & Std & Min & Lokalisierung \\
\hline 1621 & 5 & 20 & & & Neuchâtel \\
\hline 1621 & 5 & 21 & & & Neuchâtel \\
\hline 1622 & 3 & 1 & & & Engadin, GR \\
\hline 1622 & 7 & 24 & 22 & & Unterengadin, GR \\
\hline 1623 & 2 & 20 & & & Chiesa, I \\
\hline 1635 & 6 & 11 & 23 & & Zürich \\
\hline 1635 & 6 & 21 & 23 & & Zürich \\
\hline 1642 & 6 & 3 & 21 & 30 & Piz d'Err, GR \\
\hline 1648 & 11 & 23 & & & Neuchâtel \\
\hline 1650 & 1 & 8 & & & Buchs, SG \\
\hline 1650 & 5 & 6 & & & Aesch - Basel \\
\hline 1650 & 9 & 7 & & & Basel \\
\hline 1650 & 9 & 10 & 3 & & Pratteln - Basel \\
\hline 1650 & 9 & 11 & 1 & 30 & Basel \\
\hline 1650 & 10 & 18 & & & Brugg \\
\hline 1650 & 10 & 20 & 12 & & Brugg \\
\hline 1650 & 10 & 25 & 11 & & Bremgarten \\
\hline 1651 & 1 & 8 & & & Basel \\
\hline 1651 & 12 & 7 & 15 & & Genève \\
\hline 1652 & 2 & 2 & & & Sax \\
\hline 1652 & 2 & 4 & & & -- \\
\hline 1652 & 2 & 14 & & & Zürich \\
\hline 1652 & 8 & 19 & & & -- \\
\hline 1652 & 10 & 13 & & & Niederurnen - Näfels, GL \\
\hline 1652 & 10 & 23 & & & Niederurnen - Näfels, GL \\
\hline 1656 & 2 & 23 & & & Basel \\
\hline 1656 & 3 & 2 & & & Blauen - Ettingen, BL \\
\hline 1661 & 1 & 9 & 21 & & Glarus \\
\hline 1661 & 2 & 10 & & & Glarus \\
\hline 1663 & 9 & 10 & 21 & & Elm, GL \\
\hline 1663 & 9 & 13 & & & -- \\
\hline 1665 & 3 & 3 & & & -- \\
\hline 1665 & 3 & 31 & & & Neuchâtel \\
\hline
\end{tabular}




\begin{tabular}{|c|c|c|c|c|c|}
\hline Jahr & Mt & Tag & Std & Min & Lokalisierung \\
\hline 1666 & 3 & 1 & & & Glarus \\
\hline 1666 & 9 & 1 & & & Bodensee \\
\hline 1666 & 9 & 11 & & & Arbon, TG \\
\hline 1668 & 4 & 20 & 14 & & Glarus \\
\hline 1669 & 9 & 30 & 12 & 45 & Strasbourg, F \\
\hline 1670 & 7 & 6 & 1 & & Neuchâtel \\
\hline 1670 & 9 & 18 & & & Glarus \\
\hline 1670 & 12 & 2 & & & Zürich \\
\hline 1671 & 5 & 22 & & & Sax, in Sennwald, SG \\
\hline 1672 & 1 & 9 & 14 & & Hohensax, in Sennwald, SG \\
\hline 1672 & 1 & 12 & 10 & 30 & Sax, in Sennwald, SG \\
\hline 1672 & 5 & 12 & 10 & 30 & Buchs, SG \\
\hline 1672 & 5 & 22 & 11 & 30 & Buchs, SG \\
\hline 1672 & 12 & 2 & 14 & & Kanton Zürich \\
\hline 1674 & 11 & 6 & 8 & & - \\
\hline 1674 & 12 & 6 & 8 & & Glarus \\
\hline 1676 & 6 & 17 & 20 & & Ivrea, I \\
\hline 1677 & 12 & 13 & 6 & & St. Gallen und Gais, AR \\
\hline 1679 & 1 & 2 & 2 & 30 & St. Gallen \\
\hline 1680 & 6 & 24 & & & Schweiz \\
\hline 1680 & 7 & 24 & & & St. Aubin, FR \\
\hline
\end{tabular}




\section{Bibliografie}

\subsection{Manuskripte}

AEGE Archives d'Etat de Genève

Ms hist. no 19 (ancien 141bis): Annales de Genève 1603-1766 (Liste der Syndics mit annalistischen Notizen, Kompilation)

BSB Bayerische Staatsbibliothek München

- Kilian Leib, Rebdorf bei Ingolstadt: 4 L Impr. C. n. mss 73. Kalender mit Notizen zum Wettergeschehen. (Arbeits-Transkription in Datenbank Euroclimhist Mittelalter 1, Schwarz-Zanetti, 1992, Nr. 8858)

- Hieronymus Rose; Ingolstadt: Eph. Astr. 127. Kalender mit Notizen zum Wettergeschehen. Arbeits-Transkription in Datenbank Euroclimhist Mittelalter 1, Schwarz-Zanetti, 1992, Nr. 8885).

EAF Erzbischöfliches Archiv Freiburg im Breisgau, D

- Synopsis Annalium Monasterij S. Petri in nigra Silva O.S.B. Manuskript aus dem Erzbisch. Archiv Freiburg i. Br., Sign.: EAF, Ha 583. [2. Drittel 18. Jh.]

FAHASM Fondation des Archives Historiques de l'Abbaye de Saint-Maurice

- Bérodi, Gaspard: Chronique du chanoine Bérodi. Sign.: DIV/13/0/1. (Online-Zugang)

GFZ Geoforschungszentrum Potsdam

- Lersch, B. M. (o. Jahr): Erdbeben-Chronik für die Zeit von 2362 v. Chr. bis 1897. 19-bändige Handschrift, zitiert in Sieberg (1940). [Wurde für diese Publikation nicht direkt konsultiert.]

LAAI Landesarchiv Appenzell Innerrhoden

- Missale der Pfarrkirche Appenzell. Ohne Signatur. (Vgl. auch Edition A. von Euw, 2004.)

- Sauter-Chronik, Landesarchiv Appenzell

SbWi Schlossbibliothek Wildegg

- Stumpfchronik (Ausgabe 1586), darin handschriftlicher Eintrag von Hans Friedrich Effinger zu 2 Erdstössen am 21. (11.) September und 28. (18.) Oktober 1650.

StAAR Staatsarchiv Appenzell Ausserrhoden

- Sammlung der Geschichten, so theils auf das Land Appenzell bezug haben, anders theils auch die alte Rood und Kirchhöri Speicher betrefend ... Anno 1815 von Johann Bartholome Rechsteiner. Teil 2. Sign.: MG 26/2 
StANE Staatsarchiv Neuenburg

- Favarger: Journal 1547-1681 (avec lacunes). Sign.: Livres de raison/ Journaux LRJ

StASaR Stadtarchiv Stein am Rhein

- Kirchenbücher 1559-1638, Sign.: Ki 1

- Isaak Vetter (1692-1747), Geschichten der Stadt Stein, 7 Bde, Bü 4753: Bü 49, Bd III 1600-1699. (Kompilation)

StASH Staatsarchiv Schaffhausen

- Chronik der Stadt Schaffhausen, zusammengetragen und geschrieben durch Hans Jacob Spleiss, V. D. M. [Pfarrer, 1586-1657], ohne Signatur

- Burgauer, Hans Ludwig, Chronik 1627-1680 (ohne Signatur)

StASZ Staatsarchiv Schwyz

- Sammlung Felix Donat Kyd, Schwyz, 19. Jh., Sign.: PA 13, Slg, Kyd, Bd. 2.

StATG Staatsarchiv Thurgau

- Tauf-, Ehe- und Totenregister

- 995275

- 995257

StAZH Staatsarchiv Zürich

- Ebel, Johann Gottfried (1764-1830), Fragment einer Erdbebenkompilation (letzter Eintrag 1805): StAZH: B IX.

- Kirchenbücher im StAZH:

Benken: $\quad$ E III 013.1

Brütten: $\quad$ E III 017.1

Stammheim: E III 0117.2

Meilen: E III 075.2

Kilchberg: $\quad$ E III 062.1 und 062.2

Stäfa: $\quad$ E III 0115.3

Hirzel: E III 053

Niederglatt und Niederhasli: E III 79.1

- Kirchlicher Brief (des Antistes?) aus Zürich an die Dekane, 21. (11.) Juli 1670: E II 35 a

- Brief aus Salez vom 15. Mai 1671 von Jakob Zimmermann an den Antistes in Zürich: E II 35

- Hofmeister Salomon: E II 35.

StiAEi Stiftsarchiv Einsiedeln

- Abt Placidus, Sign.: A. HB 101

- Josef Dietrich, Einsiedeln Dietrich Joseph: Acta sive Diarium rerum memorabiliumMonasterii B. V. Einsidlensis Annis 1670 ... 1699 Ab initio Regiminis ... Augustini lidi Abbatis conscribi coepta ... Friderico Helmin Lucernensi Continuata vero per F. Josephum Dietrich Rapperswilen ... Signatur: A HB 1-11, Pfäffikon: B Tg 2. 


\section{StiAEng Stiftsarchiv Engelberg}

- Nekrologium: Cod. 26 fol. 53v

StiASG Stiftsarchiv St. Gallen

- Tagebuch des Abtes Pius 1650-1653: Bd 262 B

- Abt Gallus Alt. Diarium Illustrissimi Abbatis Galli II ab Anno 1665 usque 1672. Tomus I Stiftsarchiv St. Gallen Bd. 265

StibSG Stiftsbibliothek St. Gallen

- Abschrift der Zürcher Chronik in Jakob Twinger von Königshofen, Stiftsbibliothek St. Gallen, Codex 631; wahrscheinlich aus dem Jahr 1473.

- Die Konstanzer Chronik des Gebhard Dachers: Codex 646. (Vgl. auch die Edition von S. Wolff, 2008.)

UBB Universitätsbibliothek Basel

- Codex Beinheim, Univ. Bibl. Basel, fol. 32:

- Wettertagebuch des Basler (?) Anonymus, 1399-1405, Manuskript in der Universitätsbibliothek Basel: F. III.8, lateinisch-deutsche Arbeitstranskription in Klimadatenbank Schwarz-Zanetti, Euroclimhist Mitteleuropa, Mittelalter, Hist. Institut der Univ. Bern. Vgl. auch Thorndike.

- Philipert, J. H.: Denckwürdige historische Geschichte. Welche zarischen E: C: Rats E: C: Bürger-Schaft und Untertahnen zu Stadt und Land täglich zu getragen. 1545-1743. A.H. Basler Historische Croneck. Von Herr Jos. Heinrics Philipert. Sign.: UBB, Handschriften, VB Mscr. 07 p. 8-132

WLB Württembergische Landesbibliothek Stuttgart

- Johannes Stöffler, Tübingen: R 16 Stoe 1. Kalender mit handschriftlichen Notizen zum Wettergeschehen (Arbeits-Transkription in der Datenbank Euroclimhist Mittelalter 1, 1992, Nr. 8857)

ZHBL Zentral- und Hochschulbibliothek Luzern

- Das Familienarchiv Amrhyn. Akten, Urkunden und Briefe einer Luzerner Patrizierfamilie 16. bis 19. Jh. Bearbeitet von Josef Brülisauer, 1982. Sammelband des Franz Leonz Meyer (1656-1737) von Schauensee: Chronikalische Aufzeichnungen (1434-1689). Ohne Signatur.

ZBZ Zentralbibliothek Zürich, Handschriftenabteilung

- Ms D 269-271: Wolfgang Haller, Tagebuch in gedrucktem Kalender 1544-1578, von Zürich

- Ms B 258: Wynrechnung in Guggenbühl-Chronik, entstanden 16881692; vgl. auch Wolf (1856/57)

- Wickiana:

Ms F 23.357. Brief vom 1574, 30. Juli, Zürich.

Ms F 32, folio 19-22, Briefe 1584, mit Erdbebenbild. 


\subsection{Editionen und Sekundärliteratur}

\subsubsection{Abkürzungen}

BrG: $\quad$ Bibliotheca rerum Germanicarum

MGH SS: Monumenta Germaniae Historica Scriptores

MGH SRG: Monumenta Germaniae Historica Scriptores rerum Germanicarum in usum scholarum [Die MGH sind auch digital zugänglich:

http://bsbdmgh.bsb.Irz-muenchen.de/dmgh_new/]

- Albini, P. et al. (1994): Alcuni terremoti importanti per l'Alta Valtellina. Studi sismici in Alta Valtellina, ISMES-IRRS (CNR), n. 336. S. 25-50.

- Alexandre, P. (1984): Problèmes de méthode relatifs à l'etude des séismes médiévaux. Tremblements de terre histoire et archéologie. S. 221-235.

- Alexandre, P. (1990): Les séismes en Europe occidentale de 394 à 1259. Nouveau catalogue critique. Série Géophysique No Hors-Série.

- Alexandre, P. (1991): The seismic cataclysm of 29 March 1000: genesis of a mistake. Tectonophysics, 193, S. 45-52.

- Alexandre, P. (1994): Historical seismicity of the lower Rhine and Meuse valleys from 600 to 1525: a new critical review. Geologie en Mijnbouw 73, S. 431-438.

- Amberg, B. (1892): Beiträge zur Chronik der Witterung und verwandter Naturerscheinungen mit besonderer Rücksicht auf das Gebiet der Reuss und der angrenzenden Gebiete der Aare und des Rheins. Teil II. Jahresberichte über die Höhere Lehranstalt zu Luzern, 1889/90; 1891/92; 1896/97.

- Anhorn, B. (1873): Graw-Pünter-Krieg 1603-1629/beschrieben von Bartholomäus Anhorn; nach dem Manuskript zum ersten Male hg. von Conradin von Moor. Chur. Bündnerische Geschichtsschreiber und Chronisten, 9.

- Anhorn, B., jun. (1665): Christliche Betrachtung der vielfältigen, sich dieser Zeit erzeigenden Zornzeichen Gottes, Basel.

- Annales Admontenses (1851). MGH, SS 9, ed. W. Wattenbach, Hannover, S. 569-579.

- Annales Altahenses Maiores (1891). MGH, SRG in usum scholarum 4, ed. F. von Giesebrecht und E. von Oefele, Hannover, S. 17.

- Annales Argentinenses (1861). MGH, SS, 17, ed. P. Jaffé, Hannover, S. 86-90.

- Annales Basileenses et Colmarienses (1861), MGH, SS 17, ed. G.H. Pertz, Hannover, S. 189-270.

- Annales S. Blasii et Engelbergenses, MGH SS 17, ed. G. H. Pertz, Hannover, S. 278282. 
- Annales Brunwilarenses Anno 1152-1179 (1859), MGH SS 16, ed. G. H. Pertz, Hannover, S. 724-728.

- Annales Casinenses (1866, Neudruck 1989), MGH SS 19, ed. G. H. Pertz, Hannover, S. 302-320.

- Annales Colmarienses maiores (1861), MGH, SS, 17, ed. P. Jaffé, Hannover, S. 205.

- Annales Corbeienses (1864), Bibliotheca rerum Germanicarum 1, ed. P. Jaffe, Hannover, S. 32-65.

- Annales Einsidlenses (1839), MGH, SS 3, ed. G.H. Pertz, Hannover, S. 137.

- Annales Elwangenses (1852), MGH, SS, 10, ed. O. Abel, Hannover, S. 17-20.

- Annales Francofurtani 1306-1358 (1868). Fontes rerum Germanicarum. Geschichtsquellen Deutschlands 4, ed. J.F. Boehmer, Stuttgart 1868, S. 394-395.

- Annales Gotwicenses, MGH SS 9 (1851), ed. von W. Wattenbach, Hannover, S. $601-$ 604.

- Annales Hildesheimenses (minores). MGH SS 3 (1839), ed. G. H. Pertz, Hannover, S. 95.

- Annales Osterhovenses, MGH SS 17 (1861), ed. G.H. Pertz, Hannover, S. 537-558.

- Annales Ratisponenses, MGH SS 17 (1861), ed. G.H. Pertz, Hannover, S. 579-588.

- Annales S. Emmerammi Ratisbonensis, (1861), MGH, SS, 17, ed. P. Jaffé, Hannover, S. 571-572.

- Annales S. Stephani Frisingensis, MGH SS 13 (1881), ed. G. Waitz, Hannover, S. 5160.

- Annales Sangallenses MGH SS 1 (1826), ed. I. von Arx, Hannover, S. 82.

- Annales Wirziburgenses, MGH SS 2 (1829), ed. G.H. Pertz, Hannover, S. 238-247.

- Ardüser, H. (1877): Rätische Chronik. Hsg. J. Bott, Beilage zu den Jahresberichten der bündnerischen naturhistorischen Gesellschaft XV-XX.

- Aretius, B. (1589): SS. Theologiae problemata, sev loci communes, et miscellaneae quaestiones/authore D. Benedicto Aretio, theologo Bernensi. Editio quarta, locupletior \& emendatior superioribus ... Genevae.

- Bächtold, J., Hsg. (1876): Hans Salat, ein Schweizer Chronist und Dichter aus der ersten Hälfte des XVI. Jahrhunderts: sein Leben und seine Schriften. 1. Band. Basel.

- Baratta, M. (1901): I terremoti d'Italia. Saggio di storia geografia e bibliografia sismica italiana con 136 sismocartogrammi. Torino, Forni.

- Bayerischer Erdbebenkatalog: www.erdbeben-in-bayern.de/erdbebenkatalog/lokalbeben (Januar 2011).

- Becker, A. \& Davenport, C. (2003): Rockfalls triggered by 1356 Basle Earthquake, Terra Nova, 15 (4), S. 258-264.

- Beer, de, G. (1953): Pour le bicentenaire de l'arrivée de Gibbon à Lausanne. Revue historique Vaudoise, S. $201 \mathrm{f}$. 
- Bergmann, U. (1997): Die ehemalige Prämonstratenser Klosterkirche St. Maria und Michael Churwalden. Schweizerischer Kunstführer GSK. Serie 62, Nr. 611.

- Bernhertz, M. (1616): Terraemotus. Ein gründlicher Bericht von den Erdbeben/was dieselbige seyen/... Sampt einem Register und ordentliche Erzehlung aller (...), Nürnberg.

- Bernoldi Chronicon. MGH SS 5 (1844), ed. G. H. Pertz, Hannover. S. 385-467.

- Bernoulli, A. (1888): Die älteste deutsche Chronik von Colmar. Colmar.

(1890): Appenweiler Zusatz: Anonyme Zusätze und Fortsetzungen zu Königshofen, nach der Abschrift Erhards von Appenwiler 1120-1454. Basler Chroniken, Bd. IV. Leipzig.

(1895): Die Grösseren Basler Annalen. Basler Chroniken, Bd. 5, S. 23 und Bd. 6. (1902): S. 254. Leipzig.

(1895): Die Kleineren Basler Annalen. Basler Chroniken, Bd. 5, S. 51-57.

(1895): Die Chronik Henmann Offenburgs 1413-1445. Basler Chroniken Bd. 5. Leipzig 1895, S. 225-299. Leipzig.

(1902): Die Anonyme Chronik bei Schnitt, sammt Fortsetzung 1495-1541. Basler Chroniken, 6. Band. S. 185f. Leipzig.

(1902): Größere Basler Annalen nach Schnitts Handschrift. Basler Chroniken 6, S. 239-275. Leipzig.

(1902): Die Spätern Aufzeichnungen bei Schnitt 1400-1487. Basler Chroniken, 6. Band. Leipzig.

(1902): Die Anonyme Chronik der Mailänderkriege 1507-1516. Basler Chroniken, 6. Band. Leipzig.

(1902): Die Chronik in Ludwig Kilchmanns Schuldbuch 1468-1518. Basler Chroniken, Bd. 6. Leipzig.

- Bertholdi Annales (1844): Hsg. Pertz, G. H., MGH SS 5, Hannover. S. 264-326.

- Bertrand, E. (1756): Mémoires Historiques et physiques sur les tremblemens de terre: avec Quatre sermons à cette occasion. Vevey.

- Beuther, M. (1557): Calendarium Historicum. Tagebuch / Allerley Fürnhemer / Namhafftiger unnd merchklicher Historien / Aus vielen / inn sechserley Sprachen / alt und new beschriebnen cronicken / maeniglichem zu sonderem Lust und Nutz / mitt fleiss zusammen gebracht. / (...) Frankfurt am Main.

- Beuther, J., M. (1601): Compendium Terraemotuum. Das ist/Kurtzer Begriff/und Gründtliche verzeichnuss/Aller unnd jeder Erdbidemen/die sich von zeit der allein seligmachenden Geburt Jesu Christi unsers Erlösers und Heilandes/biss auff jetzige 1601. Jahre hin und wider in der gantzen Welt erhebt und zugetragen haben ... . Strassburg.

- Binder, [n. n.] (1905-1906): Ein poetischer Erdbebenbericht von 1498 (Saanen, Suisse). Die Erdbebenwarte Ve an., S. 226-227. Laibach. 
- Bitschnau, M. (2005): Bauarchäologie und Stadtgeschichte - Die Befunde der archäologisch-bauanalytischen Notuntersuchung in der Dompfarrkirche St. Niklaus. In Getzner, M. A., Hsg. (2009): Burg und Dom zu Feldkirch. Neue Forschungen zur Geschichte der Schattenburg in der Dompfarrkirche St. Niklaus. Schriftenreihe der Rheticus-Gesellschaft 50, in Zusammenarbeit mit der Heimatpflege und dem Museumsverein Feldkirch. Feldkirch 2009. S. 297-350.

- Boehme, H. (2004): Zeichen des Himmels. Albrecht Dürer und die Kometenfurcht. NZZ, 24. Dezember 2004. (www.nzz.ch/2004/12/24/li/articleA1JDN.html.)

- Borst, A. (1981): Das Erdbeben von 1348. Ein historischer Beitrag zur Katastrophenforschung. Historische Zeitschrift, Band 233, S. 528-569.

- Boschi, E., Guidoboni, E., Ferrari, G., Valensise, G., Gasperini, P. (1997): Catalogo dei forti terremoti in Italia dal 461 a. C. al 1990. Istituto Nazionale di Geofisica, Roma.

- Boyve, J. (1854-1858): Annales historiques du comté de Neuchâtel et Valangin depuis Jules-César jusqu'en 1722. Berne et Neuchâtel, 5 Bände.

- Brügger, C. (1876): Beiträge zur Natur-Chronik der Schweiz insbesondere der Rhätischen Alpen. Beilage zum Programm der bündner'schen Kantonsschule. 1876, 1877, 1879, 1881, 1882, 1888. Chur.

- Brunold, U. \& Muraro, J., Hsg. (2008): Necrologium Curiense. Mittelalterliche Totenund Jahrzeitbücher der Kathedrale Chur: Codices C, D, E und G des Bischöflichen Archivs Chur. Dietikon-Zürich.

- Burgauer, J. (1651): Christlicher / grundtlicher Underricht Von den Erdbidmen: Darinn Derselbigen natur / Eigenschafft / und vilfaltiger underscheid / ursprung und Haerkommen / würckung und bedeütung eigentlich beschrieben und dargethan wirt: Auss heiliger Goettlicher Schrifft / ... / biss auff den / der Anno 1601. den 8. September gefolget / erkläret und bestätiget: Durch Johann Burgower / der Artzneyen Doctoren zu Schaffhausen. Getruckt zu Zürich Durch Joh. Heinrich Hamberger ... .

- Busslied, Zwey christenlich. Vom Erdbidem dess MDCI. Jars: und anderen darauf volgenden Himmelischen zeichen: Sonderlich von dem Feuhrzeichen, so uff den tag dess Erdbidems den 8. Herpstmonats zu zwey Jahr umb im 1603 nachts umb 10 uhr allenthalben ist gesehen worden. Im Jahr 1603. o. 0. 8. B. 8. 42 und 9 Str. In Zürich. (Weller: Annalen I, Bd. 1862, S. 265.)

- Camassi, R., Santi, M., Rebez, A., Stucchi, M., Zerga, A. (o.J.): Problems and pitfalls in the compilation of comprehensive, parametric earthquake catalogues. On-lineversion, 5.2.2009.

- Campell, U. (1884): Raetiae alpestris topographica descriptio, hg. auf Veranstaltung der Schweizerischen geschichtsforschenden Gesellschaft von C. J. Kind, Basel.

- Candreia, J. (1905): Zur Chronik der Erdbeben in Graubünden bis zum Jahre 1879. Bern. 
- Cappelli, A. (1901): Lexicon Abbreviaturarum. Wörterbuch lateinischer und italienischer Abkürzungen, wie sie in Urkunden und Handschriften ... des Mittelalters ... . Weber, Leipzig.

- Carrozzo, M.T., de Visentini, G., Giorgetti, F., laccarino, E. (1973): General catalogue of Italian earthquakes. Comitato Nazionale Energia Nucleare. Roma.

- Casus monasterii Petrishusensis, MGH SS 20 (1868), Hsg. G. H. Pertz, S. 621-683. Hannover.

- CENEC-Catalogue (Grünthal et al., 2009) [The unified catalogue of earthquakes in central, northern, and northwestern Europe (CENEC) - updated and extended to the last millennium].

- Chiaverini, G. (1667): Historia della miraculosa Apparitione della Vergine Santiss. E Madre di Dio Nella Valle S. Giacomo di Campo Dolcino Data gia in Luce da Prete Guglielmo Chiaverini con altri racconti, et hora raccolta dal medesimo semplicemente con alcune lodi di nuovo aggiunte d'essa. Como.

- Chronicon Monasterii Zwifaltensis (auch: Bertholdi liber de constructione monasterii Zwivildensis), hgs. von O. Abel, MGH SS 10 (1852) S. 96-124. Hannover.

- Chronik

- aus Breisach (1863): Quellensammlung der badischen Landesgeschichte. Dritter Band. Hsg. Mone, F., J. Karlsruhe.

- des Burkard Zink 1368-1468 (1866). Chronik der deutschen Städte vom 14. ins 16. Jahrhundert, 5. Bd. Augsburg, 2. Bd. Leipzig.

- des Erhard Wahraus 1126-1445, (1865). Chronik der deutschen Städte vom 14. ins 16. Jahrhundert, 4. Bd. Leipzig.

- des Hector Mülich 1348-1487 (1892). Chronik der deutschen Städte vom 14. ins 16. Jahrhundert, 5. Bd. Augsburg, 3. Bd. Leipzig.

- des Jacob Twinger von Königshofen. 1400 (1415) (1871). Die Chroniken der deutschen Städte vom 14. bis ins 16. Jahrhundert. Achter Band. Die Chroniken der oberrheinischen Städte. Strassburg. Erster Band, 1870 und Zweiter Band, 1871. Leipzig.

- Die älteste deutsche Chronik von Colmarer (1888), hsg. von A. Bernoulli. Colmar.

- von Augsburg 1368-1406 (hsg. 1865). Die Chroniken der deutschen Städte vom 14. bis ins 16. Jahrhundert, Bd. 4. Hsg. von Frensdorff, Lexer, Roth. Leipzig.

- Closener, F. (1870): Fritsche (Friedrich) Closener's Chronik. 1362. Die Chroniken der deutschen Städte vom 14. bis ins 16. Jahrhundert. Achter Band. Die Chroniken der oberrheinischen Städte. Strassburg. Erster Band. Leipzig.

- Cononis praepositi Lausannenses Notae (1879), MGH SS 24, S. 781-793. Hsg. von G. Waitz, Hannover.

- Continuatio quae dicitur praemonstratensis, MGH, SS, 6 (1844), ed. L. Bethmann, Hannover, S. 447-456.

- Continuatio Vindobonensis, MGH, SS, 9 (1851), ed. G. Pertz, Hannover, S. 699-722. 
- Cysat, R. (1969): Collectanea Chronica und denkwürdige Sachen pro Chronica Lucernensi et Helvetiae. Erste Abteilung, Stadt und Kanton Luzern. Erster Band, Zweiter Teil. Collectanea Chronica und denkwürdige Sachen zur Geschichte der Stadt Luzern. Bearbeitet von Dr. phil. Josef Schmid. Mit zum Teil farbigen Abbildungen im Text und auf Kunstdrucktafeln. Luzern.

- Dettling, M., Hsg. (1900-1927): Schwyzerischer Geschichtskalender.

- Dierauer, J., Hsg. (1890): Chronik der Stadt Zürich. Mit Fortsetzungen. Quellen zur Schweizer Geschichte, Bd. 18. Basel.

- Diessbach, von, H., Hsg. (1849): Der Schwabenkrieg/besungen von einem Zeitgenossen Johann Lenz, Zürich. (Ist auch online zugänglich.)

- Diessenhofen, de, Heinricus und andere Geschichtsquellen Deutschlands im Mittelalter. Hsg. aus dem Nachlasse J. F. Boehmers von A. Huber. (1868.) Fontes rerum Germanicarum. Geschichtsquellen Deutschlands. Hsg. von J. Boehmer. Vierter Band.

- Dohrn-van Rossum, G. (1996): History of the hour: clocks and modern temporal orders. Chicago.

- DOM4.1: Un database di osservazioni macrosismiche di terremoti di area italiana al di sopra della soglia del danno/an intensity database of damaging earthquakes in the Italian area. http://emidius.mi.ingv.it/DOM/

- Dumur, M. B. (1907): Notes extraites des registres de l'état-civil de la paroisse de Pully. Revue Historique Vaudoise, 1907, S. 329.

- Durrer, R. (1899-1928/1971): Die Kunstdenkmäler des Kantons Unterwalden. Zur Statistik Schweizerischer Kunstdenkmäler. Kommission für das Schweizerische Landesmuseum, Zürich.

- Ebbo, Vita Ottonis episcopi Babenbergensis, MGH 5 1869, S. 588-692, hsg. von Ph. Jaffé.

- ECOS-02: Earthquake Catalog of Switzerland. http://www.seismo.ethz.ch.

- ECOS-09: Earthquake Catalog of Switzerland. http://www.seismo.ethz.ch.

- Eisbacher, G. \& Clague, J. (1984): Destructive Mass Movements in High Mountains: Hazard and Management. Geological Survey of Canada. Paper 84-16.

- EKDAG 2006: Erdbebenkatalog für Deutschland und angrenzende Gebiete - erweiterter Ahorn-Katalog 2006.

http://www.edac.biz/uploads/media/EKDAG2006_VGB_Int6.pdf

- Ellenhardi Argentinensis Annales 1261-1294, MGH SS 17 (1861), ed. P. Jaffé, Hannover, S. 118-141.

- Ellenhardi Chronicon, MGH SS 17 (1861), ed. P. Jaffé, Hannover, S. 132.

- Erdbebenkatalog Bayerns (http://www.erdbeben-in-bayern.de/erdbebenkatalog/lokalbeben) 
- EuroClimHist: http://histserver.unibe.ch/euroclimhist_w/pages/index.htm. Klima-Datenbank, Christian Pfister, Historisches Institut der Universität Bern und Oeschger Centre for Climate Change Research, Bern.

- Euw, von, A. \& Bischofberger, H. (2004): Das Appenzeller Missale: eine illuminierte Handschrift des 12. Jahrhunderts. Innerrhoder Schriften, 11. Appenzeller Volksfreund, Appenzell.

- [Fabri, Felix] Felicis Fabri Monachi VImensis Historia Sueuorum (1604): Sueuicarum rerum Scriptore aliquot veteres ex recensione Melchioris Haiminsfeldii Goldasti, Francof. 1604. In Wackernagel, Wilhelm: Das Erdbeben von 1356 in den Nachrichten der Zeit und der Folgezeit bis auf Christian Wurstisen. Basel im vierzehnten Jahrhundert. Geschichtliche Darstellungen zur fünften Säcularfeier des Erdbebens am S. Lucastage 1356 (1856), S. 237-239.

- Fäh, D.; Gisler, M.; Jaggi, B.; Kästli, P.; Lutz, T.; Masciadri, V.; Matt, C.; Mayer-Rosa, D.; Rippmann, D.; Schwarz-Zanetti, G.; Tauber, J.; Wenk, T. (2009): An interdisciplinary revision of the 1356 Basel Earthquake. Geophysical Journal International, 178, S. 351-374.

- Fäh, D., Schatzmann, R., Oprsal I. (2008): Augusta Raurica - Spuren eines Erdbebens in der Mitte des 3. Jahrhunderts? Nachbeben. Eine Geschichte der Erdbeben in der Schweiz, Bern.

- Fäh, D., Steimen, S., Oprsal, I., Ripperger, J., Wössner, J., Schatzmann, R., Kästli, P., Spottke, I. and P. Huggenberger (2006): The earthquake of 250 A.D. in Augusta Raurica, a real event with a 3D site-effect? Journal of Seismology, Vol. 10, No. 4, S. 459-477.

- Five Millennium Catalog of Solar Eclipses (http://eclipse.gsfc.nasa.gov/SEcat5/ SE-1299--1200.html)

- Flores temporum Auctore Fratre Suevico ordinis minorum. Hsg. von O. Holger-Egger. MGH SS, 24 (1879), S. 230-250.

- Gaillard, A. (1996): L'église Saint-Jean Ardon-Magnot. Sion. (Conseil de gestion de la Paroisse d'Ardon-Magnot).

- Gamper, R. (1984): Die Zürcher Stadtchroniken und ihre Ausbreitung in die Ostschweiz. Forschungsgeschichte, Ueberlieferung, Analyse der Chroniktexte. Mitteilungen der Antiquarischen Gesellschaft in Zürich, Band 52, Heft 2 (148. Neujahrsblatt).

- Geschichtsquellen des deutschen Mittelalters - Repertorium Fontium Historiae Medii Aevi (www.repfont.badw.de).

- Gesta episcoporum Mettensium, MGH SS 10, 1852. Ed. G. Waitz, Hannover, S. 531551.

- Ghönneirus, C. (1585): Chronick, Geschichte und Zeitbuch aller nammhafftigsten und gedechtnußwierdigsten geystlichen und weltlichen Sachen oder Handlungen von anbegin der Welt nach erschaffung des ersten Menschen, biß auff das gegenwertige jar 
Christi 1585. verlengt: Darinn beyde Gottes und der Welt lauff, Hendel, Art, Wort, Werck, Thun, Lassen, Wesen und Leben ersehen und begriffen wirdt.../Weylandt durch Sebastian Francken... biß auff die Regierung... Keysers Carls des V. ... angefangen. Jetzt aber durch Calonium Ghönneirum... biß auff das 1585. jare... gemehret. Ohne Ort. [Ghönneirus ist das Pseudonym für den Basler Nikolaus Höniger.]

- Giessberger, H. (1922): Die Erdbeben Bayerns. I. Teil. Abhandlungen der Bayerischen Akademie der Wissenschaften. Mathematisch-physikalische Klasse, XXIX. Band 6, Abhandlung. Verlag der Bayerischen Akademie der Wissenschaften. München.

- Gils, Van, J., \& Leydecker, G. (1991): Erdbebenkatalog für die Bundesrepublik Deutschland mit Randgebieten für die Jahre 1000-1981. Geologisches Jahrbuch. Hsg. von der Bundesanstalt für Geowissenschaften und Rohstoffe und den Geologischen Landesämtern in der BRD. Hannover.

- Gisler, M. (2007a): Göttliche Natur? Formationen im Erdbebendiskurs der Schweiz des 18. Jahrhunderts. Zürich.

- Gisler, M., Fäh, D., Giardini, D. (ed.) (2008a): Nachbeben. Eine Geschichte der Erdbeben in der Schweiz, Bern.

- Gisler, M., Fäh, D., Masciadri, V. (2007b): „Terrae motus factus est“: Earthquakes in Switzerland before A.D. 1000. A Critical Approach. Natural Hazards, 43, S. 63-79.

- Gisler, M. \& Masciadri, V. (2008b): Erdbeben in der Schweiz in der Spätantike und im Frühmittelalter. Nachbeben. Eine Geschichte der Erdbeben in der Schweiz, Bern, S. 11-20.

- Gisler, M., Schwarz-Zanetti, G., Fäh, D. und Kästli, P. (2008c): Das Erdbeben von Basel 1356: eine historisch-archäologische Annäherung. Nachbeben. Eine Geschichte der Erdbeben in der Schweiz, Bern, S. 41-49.

- Gisler, M., Weidmann, M., Fäh, D., Kästli, P., Schwarz-Zanetti, G., Deichmann, N. (2005): Erdbeben in Graubünden. Vergangenheit, Gegenwart, Zukunft.

- Goethe, von, J. (1950/1977): Faust II. Gesamtausgabe der Werke und Schriften in 22 Bänden. Cotta, Stuttgart, Artemis, Zürich.

- Gollnick, U. (2001): Die Burgruine Gams. Archäologische Ausgrabungen und Bauanalyse. Mit einem Abschnitt über das Erdbeben 1295 von G. Schwarz-Zanetti. Mittelalter. Zeitschrift des Schweizerischen Burgenvereins. 6. Jahrgang, [Basel] 2001/1, S. 26-35. Und in: Werdenberger Jahrbuch 2002. Historisch-Heimatkundliche Vereinigung des Bezirks Werdenberg. 15. Jahrgang, 2001.

- Goswin, P. (1880): Chronik des Stiftes Marienberg. Hsg. von Basilius Schwitzer. Innsbruck 1880. Tirolische Geschichtsquellen. Bd. II.

- Grabner-Haider, A. (1969, 1979): Praktisches Bibellexikon. Herder-Lexikon. Freiburg, D.

- Grimm, J. \& W. (1854-1960): Deutsches Wörterbuch, Hirzel Verlag, Leipzig. Und: http://germazope.uni-trier.de/Projects/WBB/woerterbuecher/dwb/ wbgui?lemid=GA00001 
- Groh, D., Kempe, M., Mauelshagen, F. (2003): Naturkatastrophen: Beiträge zu ihrer Deutung, Wahrnehmung und Darstellung in Text und Bild von der Antike bis ins 20. Jahrhundert. Literatur und Anthropologie, Bd. 13. Narr, Tübingen.

- Gropp, I. (1736): Aetas Mille Annorum Antiquissimi et Regalis Monasterii B.M.V. in Amorbach. Ord. S. Bened. In Archi-dioecesi Mogunt. (...). Weidmann, Frankfurt.

- Gross, J. (1614): Baszler Erdbidem, so sich innerthalb sechshundert Jahren, in und umb die Statt und Landschafft Basel erzeigt haben/Mit sampt einem schrifftmessigen Bericht, was der Erdbidem für ein Bewegung seye/woher er entstehe/und was derselbige bedeute. Den einfältigen christen zu gutem gestellt/durch Johan. Georg. Grossen. Basel.

- Gross, J. (1624): Kurtze Bassler Chronick: Oder/Summarischer Begrieff aller denckwürdigen Sachen und Händeln/so sich von vierzehendhundert Jahren biss auff das M.DC. XXIV Jahr/in und bey der Statt Basel/mit grossen Potentaten/und anderen benachbarten Fürsten und Herren/zu Kriegs: und Friedenszeiten zugetragen: Und was sonsten für Gutthaten von Gericht Gottes dero begegnet: So wol auss geschriebenen/alss getruckten Chronicken/und anderen warhafftigen Berichten/zusammen getragen Durch Johann Gross/Kyrchendiener daselbsten. Getruck zu Basel Durch Johann Jacob Genath.

- Grotefend, H. (1991, 13. Aufl.): Taschenbuch der Zeitrechnung des deutschen Mittelalters und der Neuzeit. Hahn, Hannover. Auch online zugänglich:

http://www.manuscripta-mediaevalia.de/gaeste/grotefend/grotefend.htm.

- Grünthal, G., ed. (1998): European Macroseismic Scale 1998 (EMS-98), Cahiers du Centre Européen de Géodynamique et de Séismologie 15, Helfent-Bertrange (Luxembourg).

- Grünthal, G., Fischer, J. (2001): Eine Serie irrtümlicher Schadenbeben im Gebiet zwischen Nördlingen und Neuburg an der Donau vom 15. bis zum 18. Jahrhundert. Mainzer Naturwissenschaftliches Archiv, 39, S. 15-32.

- Guéneau de Montbelliard, P. (1761): Liste Chronologique des Eruptions de Volcans, des tremblements de terre, de quelques faits météorologiques les plus remarquables, des cometes, des maladies pestilentielles, \& jusqu'en 1760, tirée des Mémoires des Académies de l'Europe, des Ouvrages périodiques, des Histoires générales \& des Relations particulieres. Collection académique, composée des Mémoires, actes ou Journaux des plus célébres Académies \& Sociétés Littéraires Etrangères, S. 488-681. Dijon.

- Guidoboni, E. (1984): 3 Janvier 1117: Le tremblement de terre du Moyen Age Roman, aspects des sources. Tremblements de terre histoire et archéologie. Actes du colloque. Préface de Haroun Tazieff. 2. 3. 4. Novembre 1983. Centre de recherches archéologiques. Association pour la promotion et la diffusion des connaissances archéologiques. Valbonne 1984. S. 119-139. 
- Guidoboni, E., Comastri, A., Boschi, E. (2005a): The „exceptional“ earthquake of 3 January 1117 in the Verona area (northern Italy): A critical time review and detection of two lost earthquakes (lower Germany and Tuscany). Journal of Geophysical Research, 110, B12309. $20 \mathrm{~S}$.

- Guidoboni, E. \& Comastri, A. (2005b): Catalogue of earthquakes and tsunamis in the Mediterranean area from the $11^{\text {th }}-15^{\text {th }}$ century. Milano: INGV, Istituto Nazionale di Geofisica e Vulcanologia. S. 84-130.

- Guidoboni, E., Ferrari, G., Mariotti, D., Comastri, A., Tarabusi, G. and Valensise, G. (2007): CFTI4Med, Catalogue of Strong Earthquakes in Italy (461 B.C. -1997) and Mediterranean Area (760 B.C.-1500). INGV-SGA. Available from

http://storing.ingv.it/cfti4med/.

- Gutscher, D., Ueltschi A., Ulrich-Bochsler, S. et al. (1997): Die St. Petersinsel im Bielersee - ehemaliges Cluniazenserpriorat. Bericht über die Grabungen und Bauuntersuchungen von 1984-1986. Berner Lehrmittel- und Medienverlag 1997, S. $114 \mathrm{f}$.

- Gutzwiller, H. (2009): Kalender. Historisches Lexikon der Schweiz, online.

- Haffner, F. (1666): Der klein Solothurner Allgemeine Schaw=Platz Historischer Geist= auch Weltlicher vornembsten Geschichten und Händlen ... . Bernhardt, Solothurn.

- Hammerl, C. (1992): Das Erdbeben vom 25. Jänner 1348. Rekonstruktion eines Naturereignisses. Diss. Univ. Wien, $250 \mathrm{~S}$.

- Hammerl, C. (1995): Erdbeben 4. Mai 1201. MIÖG 103, 1995, 350. (Mitt. des Inst. für Oesterr. Geschichtsforschung).

- Hammerl, C., \& Lenhardt, W. (1997): Erdbeben in Oesterreich. Graz.

- Haltmeyer, M. (1683): Beschreibung der Eidgnössischen Statt St. Gallen Gelegenheit/Geschichten und Regiment. Wie auch des Lebens Herrn D. von Watt/gewesenen Burgermeisters daselbst. Redinger, St. Gallen.

- Heer, G. (1975): Aus der Vergangenheit von Kloster und Tal Engelberg 1120-1970. Verlag Benediktinerkloster Engelberg. Engelberg.

- Heiligensetzer, L. (2001): Alexander Bösch. Liber familiarium personalium, das ist, Verzeichnus wass sich mit mir, und der meinigen in meiner hausshaltung, sonderliches begeben und zugetragen hatt. In: Selbst-Konstruktion. Schweizerische und Oberdeutsche Selbstzeugnisse 1500-1850. Hsg. von Kaspar von Greyerz und Alfred Messerli. Redaktion Fabian Brändle. Band 1.

- Heimo (1852): Chronographia (ex codice Augutano), ed. by G. H. Pertz, MGH Scriptores, Band 10, S. 8-11. Hannover.

- Heinzmann, J. (1795): Kleine Schweizer-Chronik. Zweyter Theil. Enthält die Ereignisse seit 1700 bis 1801 mit Einschluss der Revolutionsgeschichte von Helvetien. Bern. 
- Henne von Sargans, A., Hsg. (1861): Die Klingenberger Chronik, wie sie Schodoler, Tschudi, Stumpf, Guilliman und Andere benützten, nach der von Tschudi besessenen und vier anderen Handschriften zum erstenmal ganz, und mit Parallelen aus gleichzeitigen ungedruckten Chroniken. Gotha.

- Herimanni Augiensis Chronicon anno 1-1054 (1844). MGH SS 5, Hsg. G. H. Pertz, Hannover.

- Historisches Lexikon der Schweiz, http://hls-dhs-dss.ch.

- Hoff, Von, K. (1840-1841): Chronik der Erdbeben und Vulkanausbrüche. Mit vorausgehender Abhandlung über die Natur dieser Erscheinungen. Erster Theil. Vom Jahre 3460 vor, bis 1759 unserer Zeitrechnung. Zweiter Theil. Vom Jahre 1760 bis 1805, und von 1821 bis 1832 n. Chr. Geb., Gotha.

- Hottinger, J. (1651): Quaestiones quaedam De Terraemotu ex Hebraeorum atque Arabum Scriptis erutae (...). Bodmeri, Tiguri.

- Hottinger, J. (1665): „Speculum Helvetico Tigurinum pentagonon, quo, breviter \& succincte, de Helvetiorum, Tigurinorum cumprimis, agitur statu, .... . J. G. Simler, Zürich.

- Hölz, T. (1993): Ein bisher unbekanntes Erdbeben im Jahre 1527. Zeitschrift für Württembergische Landesgeschichte. 52. Jg., S. $463 \mathrm{f}$.

- Hülsen, von, A. (1993): Verona, 3. Januar 1117. Möglichkeiten und Unsicherheiten der interdisziplinären Erdbebenforschung. Hist. Anthropol., 1(2), S. 218-234.

- Im-Thurn, E., \& Harder, H. W., Hsg. (1844): Chronik der Stadt Schaffhausen. Verlag der Brodtmann'schen Buchhandlung, Schaffhausen.

- INGV-Liste (2000): Interne Erdbebenliste vom Istituto Nazionale di Geofisica e Vulcanologia in Mailand an den SED, Oktober 2000.

- Journal de Genève (3. August, 1855: Anonyme Erdbeben-Kurzkompilation, ohne Titel, S. 1.)

- Kaenel, von, H.-M. (1980): Eine mittelalterliche Emailfibel von der Burg Fenis. Archäologie der Schweiz, 3, 1980, 2, S. 123-125.

- Keckermann, B. (1611): Contemplatio gemina prior, ex generali physica de loco altera, ex speciali de terrae motu; Potissimum illo stupendo, qui fuit Anno 1601, mense Septembri. Scripta à Bartholomaeo Keckermanno et nunc tertio edita. Hanovieae. Apud Guilielmum Antonium.

- Keferstein, C. (1826): Versuch eines chronologischen Verzeichnisses der Erdbeben und vulkanischen Ausbrüche seit Anfang unserer Zeitrechnung. Zeitung für Geognosie, Geologie und innere Naturgeschichte der Erde. Weimar.

- Kessler, J. (1866): Johannes Kesslers Sabbata: Chronik der Jahre 1523-39. Hrsg. E. Goetzinger, Scheitlin \& Zollikofer, St. Gallen. 
- Keyssler, J. (1751): Johann Georg Keysslers, der Königlich Grossbrittannischen Societät der Wissenschaften Mitglieds, Neueste Reisen durch Deutschland, Böhmen, Ungarn, die Schweiz, Italien und Lothringen, worinnen der Zustand und das Merkwürdigste diese Länder beschrieben, und vermittelst der Natürlichen, Gelehrten und Politischen Geschichte, der Mechanik, Maler-, Bau- und Bildhauerkunst, Münzen und Alterthümer, wie auch mit verschiedenen Kupfern erläutert wird. Neue und vermehrte Auflage, welche mit Zusätzen und mit einer Vorrede von dem Leben des Verfassers begleitet hat. M. Gottfried Schütze. (...) Hannover 1751. Im Verlage sel. Nicolai Försters und Sohns Erben Hof-Buchhandlung.

- Klauber, J. R. (1617): Nachtigall/Das ist: Ein schöne Historische Beschreibung/von den sechs Straffen/mit welchen der Herr unser Gott/die herrliche/und in aller Welt hochberümbte Statt Basel: als mit Auffruhr/Krieg/Thewrung/Sterbendt/Fewrs: und Wassersnoth/auch erschrockenlichen Erdbidmen/von fünffhundert Jahren hero/ heimgesucht hat. Hergegen: Was er derselbigen auch widerum für Gutthaten erzeigt hat: Auff das fleissigste zusammen gezogen/durch Johann Rudolff Klauber/Notarius und Burger zu Basel. 1617. Gedruckt zu Basel/bey Jacob Trew.

- Klingenberger Chronik, die, wie sie Schodoler, Tschudi, Stumpf, Guilliman und Andere benützten, nach der von Tschudi besessenen und vier anderen Handschriften zum erstenmal ganz, und mit Parallelen aus gleichzeitigen ungedruckten Chroniken herausgegeben von Dr. Anton Henne von Sargans. Gotha 1861.

- Knape, J. \& Wuttke, D. (1990): Sebastian-Brant-Bibliographie: Forschungsliteratur von 1800 bis 1985 . Tübingen.

- Kopp, C. (1861): Résumé des phénomènes les plus remarquables qui se sont passés à Neuchâtel dans le 16me siècle de l'an 1500 à 1600. Bulletin de la Société des Sciences naturelles de Neuchâtel, t. 5.

- Lambert, J., Winter, T., Dewez, T. \& Sabourault, P. (2005): New hypotheses on the maximum damage area of the 1356 Basel Earthquake (Switzerland). Quaternary Science Reviews, this issue (doi:10.1016/j.quascirev.2004.02.019).

- Lancaster, A. (1901): Les tremblements de terre en Belgique. Annuaire météorologique de l'Observatoire Royal de Belgique, S. 194-228.

- Langenbeck, R. (1892): Die Erdbebenerscheinungen in der oberrheinischen Tiefebene und ihrer Umgebung. Geographische Abhandlungen aus den Reichslanden ElsassLothringen. Hsg. G. Gerland.

- Lavater, J. (1635): Thesis de terrae motus. Tiguri. [In der ZBZ mit falscher Signatur im Katalog (VI 55 a 26); richtig ist VI 55 a 44.]

- Leydecker, G. (1986): Erdbebenkatalog für die Bundesrepublik Deutschland mit Randgebieten für die Jahre 1000-1981. Geologisches Jahrbuch; Reihe E, Geophysik, Heft 36. Ed. Bundesanstalt für Geowissenschaften und Rohstoffe und Geologische Landesämter in der BRD. Hannover. 
- Leydecker, G. \& Brünning, H. (1988): Ein vermeintliches Schadenbeben im Jahre 1046 im Raum Höxter und Holzminden in Norddeutschland - Über die Notwendigkeit des Studiums der Quellen historischer Erdbeben. Geologisches Jahrbuch, E 42, Hannover, S. 119-125.

- Liebenau, v., T. (1905): Gilg Tschudis Beschreibung des Kappelerkrieges. Archiv für Schweizerische Reformationsgeschichte I, 40.

- Lindiner, J. (1711): Memorabilia Urbis et Agris Tigurini.

- Luff, R. \& Steer, G., Hrsg. (2003): Das „Buch der Natur“, Konrad von Megenberg. Bd. 2. Kritischer Text nach den Handschriften. Niemeyer, Tübingen.

- Luginbühl, R. (1908-1910): Heinrich Brennwalds Schweizerchronik. Zwei Bände. Quellen zur Schweizer Geschichte., Abt. 1, Chroniken, n. F., 2. Basel, Basler Buchund Antiquariatshaus.

- Lycosthenes, C. (1557): Prodigiorum ac ostentorum chronicon quae praeter naturae ordinem, motum et operationem, et in superioribus \& his inferioribus mundi regionibus, ab exordio mundi usque ad haec nostra tempora, acciderunt. (...) Petri, Basel.

- Mallet, E. (1845): Fasciculus temporis. Chronica Monasterii S. Victoris Gebennensis? La plus ancienne Chronique de Genève. 1303-1335. Mémoires et documents publiés par la société d'histoire et d'archéologie de Genève.

- Martins, I. \& Mendes V. (2001): Contribuiçao para o estudo da sismicidade da regiao oeste da peninsula iberica, Universidade de Lisboa, Instituto Geofisico do Infante D. Luis, Publ. N 25, 67 S.

- Masciadri, V. (2008): Eine Insel im Meer der Geschichten. Untersuchungen zu Mythen aus Lemnos. Potsdamer Altertumswissenschaftliche Beiträge (PawB), Bd. 18. Hsg. Barcelo et al., Stuttgart.

- Mayer-Rosa, D. \& Cadiot, B. (1979): Ein Ueberblick über das Erdbeben von Basel 1356: Basisdaten. Ritsema, A. R.: European Catastrophic Earthquakes. Tectonophysics, 53: S. 325-333: A review of the 1356 Basel Earthquake: Basic Data.

- MECOS-99 (1999): Macroseismic Earthquake Catalog of Switzerland, ed. Schweizerischer Erdbebendienst, ETH Zürich, http://www.seismo.ethz.ch/products/catalogs/mecos/ (URL 19. August 2008).

- Meghraoui, M., Delouis, B., Ferry, M., Giardini, D., Huggenberger, P., Spottke, I. \& Granet, M. (2001): Active normal faulting in the Upper Rhine Graben and paleoseismic identification of the 1356 Basel earthquake, Science, 293, S. 2070-2073.

- Meier, M. (2003): Zur Wahrnehmung und Deutung von Naturkatastrophen im 6. Jahrhundert n. Chr. Naturkatastrophen. Beiträge zu ihrer Deutung, Wahrnehmung und Darstellung in Text und Bild von der Antike bis ins 20. Jahrhundert. Narr, Tübingen. Hsg. Groh, D., Kempe, M., Mauelshagen, F. S. 45-64. 
- Merian, P. (1834): Ueber die in Basel wahrgenommenen Erdbeben: nebst einigen Untersuchungen über Erdbeben im Allgemeinen, Basel: A. Wieland.

- Merz, W. (1904): Werner Schodolers des Jüngern Tagebuch [Bremgarten]. Taschenbuch der historischen Gesellschaft des Kantons Aargau für das Jahr 1904. S. 77 164.

- Meyer, J. (1650): Oratio de terraemotu: ob periculossimas patriae concussiones illa A. O. R. MDCL ... . (Universitätsbibl. Basel: A 100 MAG Hp VII 38: 6.) Meier, Basel.

- Meyer, W. (2006a): Da verfiele Basel überall. Das Basler Erdbeben von 1356. Mit einem geologischen Beitrag von Hans Peter Laubscher. 184. Neujahrsblatt. Herausgegeben von der Gesellschaft für das Gute und Gemeinnützige, Basel.

- Meyer, W. (2006b): Das Basler Erdbeben von 1356 - Verlauf und Bewältigung einer Katastrophe. Mittelalter, Moyen Age, Medioevo, Temp medieval. Zeitschrift des Schweizerischen Burgenvereins. 11. Jahrgang 2006/3. S. 125-132.

- Milne, J. (1899): A catalogue of destructive earthquakes, A.D. 7 to A.D. 1899. British Association for the advancement of Science, London.

- Mone, F. (1863): Quellensammlung der badischen Landesgeschichte. Dritter Band, Macklot, Karlsruhe.

- Montandon, F. (1942-43): Les séismes de forte intensité en Suisse. Revue pour l'étude des calamités. Bulletin de l'Union Internationale de Secours. Genève.

- Moser, A. (1998): Die Kunstdenkmäler des Kantons Bern. Landband II. Der Amtsbezirk Erlach. Der Amtsbezirk Nidau 1. Teil. Hsg. von der Ges. für Schweizerische Kunstgeschichte. Wiese, Basel.

- Mühll, Von der, T. (1975): Der Donnerstein von Ensisheim. Birkhäuser, Basel.

- Müller, C. (1956): Die Burgen in der Umgebung von Basel und das Erdbeben von 1356. Basler Zeitschrift für Altertumskunde, Sonderdruck, Bd. 55.

- Müller, J. (1824): Merkwürdigkeiten der Stadt Nördlingen nebst einer Chronik mit lythographischen Zeichnungen. Beck, Nördlingen.

- Müllner, J. (1984): Die Annalen der Reichsstadt Nürnberg, 1623, Teil II: 1351-1469. Hsg. von Gerhard Hirschmann. Stadtarchiv Nürnberg.

- Münster, S. (1544): Cosmographia universalis. Henrichum Petri, Basel.

- Mutius, U. (1539): De Germanorum prima origine, moribus, institutis, legibus ... libri Chronici XXXI [...]. Heinrich Peter, Basel.

- Naef, A. (1850): Chronik oder Denkwürdigkeiten der Stadt und Landschaft St. Gallen. Mit Inbegriff der damit in Verbindung stehenden Appenzellischen Begebenheiten. Von den ältesten Zeiten bis auf das Jahr 1848. St. Gallen.

- Naegele [o. N.] (1911): Ein Erdbeben in Süddeutschland in der Weihnachtsoktav 1523. Mitteilungen zur Geschichte der Medizin und Naturwissenschaften, t. 10.

- Niermeyer, J. (1954-76): Mediae latinitatis lexicon minus. Brill, Leiden. 
- Nöggerath, J. (1870): Die Erdbeben im Rheingebiet. Verhandlungen des naturhistorischen Vereines der preussischen Rheinlande und Westphalens, Bd. 27, S. 91-111. Bonn.

- Nota Curiensis (1888): MGH Necrologia, 1, 623. Hsg. L. Baumann.

- Notae Diessenses (1861): MGH SS 17, S. 324-327, ed. G. Pertz, Hannover.

- Ortelli, L. \& Albini, P. (1998): Investigation on the 1601 and 1616, Engelberg earthquakes. Internal report for the BEEDC project, IRRS-CNR, Milano, $10 \mathrm{~S}$.

- Ovidius Naso, P. (1998): Metamorphoses. Hsg. von Thassilo von Scheffer. Diogenes, Zürich.

- Paracelsus, T. (1531): Durch den Hochgelerten herrn Paracelsum DoctoremUszlegung der Erdbidem beschehen nach Ussganng des Cometens/in den alpischen birgen/Im M.D.XXXi. Oktober, St. Gallen. Zürich, Froschauer. (http://www.ubs.sbg.ac.at/sosa/rara/Paracelsus2.jpg)

- Payot, P. (1950): Au royaume du Mont-Blanc. Bonneville, Plancher.

- Perrey, A. (1843): Mémoire sur les tremblements de terre ressentis en France, en Belgique et en Holland depuis le IVe siècle jusqu'à nos jours. Mémoires couronnés et mémoires des savants étrangers. Publiés par l'Académie royale des sciences, des lettres et des beaux-arts de Belgique, Bruxelles.

- Perrey, A. (1845): Mémoire sur les tremblements de terre dans le Bassin du Rhin. Mémoires couronnés et mémoires des savants étrangers. Publiés par l'Académie royale des sciences, des lettres et des beaux-arts de Belgique, Bruxelles.

- Pfister, C. (1984): Witterungsdatei Climhist. Schweiz 1525-1864. Meteotest, Bern.

- Pfister, C., Dietrich-Felber, U., Burri M., Horat-Gurfinkel V., eds. (2009): Euro-Climhist. A Data-Base on past Weather and Climate in Europe and its Human Dimension. Historisches Institut der Universität Bern.

http://histserver.unibe.ch/euroclimhist_w/pages/index.htm

- Plattner, P. (1887): Ulrici Campelli, Historia Raetica. Tomus I. Hsg. auf Veranstaltung der Schweizerischen Geschichtforschenden Gesellschaft. Basel, Verlag von Felix Schneider.

- Poli, De, C. \& Ghilardi, S. (1988): Considerazioni geomorfologiche sulla dinamica evolutiva della frana di Piuro (1618). Boll. Centro Studi Storici della Valtellina.

- Ragor, J. H. (1578): Von den Erdbidem Ein Grundlicher bericht/was dieselbigen seyen/ auss was ursach sie enstanden/und auff was end hin sie beschehen: auss heiliger Göttlicher Geschrifft/und auss der lehr der Philosophen zusammen gezogen. Sampt einem volkomnen Register der fürnemsten Erdbidem/von denen man in Historien liset: unnd in ein Büchlein verfasset. Mit einer Vorred/darin angezeigt wirt/wie sich die gleubigen in dieser letsten bösen zeit halten sollen/dass sie sich an jhrer Seel nicht versaumen. Von Johan Huldrich Ragor/Predicanten zu Hertzogenbuchsy aller erst an tag geben. Getruckt zu Basel bey Perter Perna. Mit Röm. Keys. May. Gnad und Freyheit. Anno M.C.LXXVIII. 
- Rahn, J. H. (1690): Eidgnössische Geschicht-Beschreibung: das ist Kurtzer Begriff aller in den loblichen XIII. und zugewandten Orthen der Eidtgnoszschafft, wie auch dero nächst angrentzenden Landen sint dem Anfang des helvetischen Namens biss auf das lauffende 1690 Jahr, vorgegangener Sachen mit möglichsten Fleiss und Unpartheylichkeit aus einer grossen Anzahle getruckter und geschriebener Autorum/zusammen getragen durch Johann Heinrich Rahn. Zürich.

- Rasch, J. (1591): Erdbidem Chronik/Nach art eines Calenders/sambt einem kurtzen bericht und Catalogo Autorum. Darinn allerley Erdbiden und Erdkluefften/vor Christi Geburt 1569 und sovil deren biss auff diss 1591. Jar her beschrieben: Diese seyen von jnnerlichem Erdrich herauss/oder vom Himmel herab/von Lufft/Wind/und Wetter verursacht/Darauss zusehen und zulernen/was desto troestlicher sich menniglich darnach zurichten hat. Durch Johann Raschen in Wien also zusammengebracht und an tag geben. Gedruckt zu München/bei Adam Berg.

- Reicke, D. (1994): Beobachtungen zur Baugeschichte der Basler „Pfalz“ anlässlich der Sanierung 1994/95. www.archaeobasel.ch/Bucherstand/Jahresbericht/JB94/ 94Kapitel10.pdf - S. 113f. (20.11.2002).

- Rem, W. (1896): Cronica newer Geschichten. Die Chroniken der schwäbischen Städte, Bd. 5. Die Chroniken der deutschen Städte, Bd. 25.

- Rhenanus, B. (1531): Rerum Germanicarum libri tres. Basel: in officina Frobeniana.

- Rohr, C. (2007): Extreme Naturereignisse im Ostalpenraum. Naturerfahrung im Spätmittelalter und am Beginn der Neuzeit. Umwelthistorische Forschungen, Bd. 4., Hsg. Grewe \& Knoll, Böhlau, Köln.

- Rothé, J.-P., \& Schneider, G. (1968): Catalogue des tremblements de terre du Fossé Rhénan (1021-1965). Ed. Institut de Physique du globe de Strasbourg/Landeserdbebendienst Baden-Württemberg, Stuttgart.

- Rummelen, Van, F. (1945): Overzicht van de tusschen 600 en 1940 in Zuid-Limburg en omgeving waargenomen aardbevingen. Mededeelingen van het Geologisch Bureau voor het Mijngebiet te Heerlen, behoorende bij het jaarsverslag over 1942 en 1943.

- Ruppert, P. (1891): Das alte Konstanz in Schrift und Stift. Die Chroniken der Stadt Konstanz. Münsterbau-Verein Konstanz.

- Rütiner, J. (1996): Johannes Rütiner. Diarium 1529-1539. Lateinischer Text und Übersetzung. Hsg. E. Rüsch. St. Gallen.

- Rüttener, E. (1995): Earthquake Hazard Evaluation for Switzerland. Matériaux pour la Géologie de la Suisse. Commission Suisse de Géophysique. Géophysique Nr. 29. Zürich.

- Salat, J. (1986): Reformationschronik 1517-1534. Text Band 2. 1528-1534. Bearbeitet von Ruth Jörg. Selbstverlag der Allgemeinen Geschichtsforschenden Gesellschaft der Schweiz. Bern. 
- Santschi, C. (1966): Les annales du notaire montheysan Claude Revilliodi 14901525. Edition, introduction et commentaire. Vallesia, Band 23, 1968, S. 31-69.

- Scaramellini, G., Kahl, G. \& Falappi G. (1988): La frana di Piuro del 1618. Storia e immagini di una rovina. Piuro.

- Schaller-Donauer, A. (1937?): Chronik der Naturereignisse im Urnerland 10001800. Separatdruck aus der Gotthard-Post. Buchdruckerei Altdorf, Gamma \& Cie.

- Schaltegger, F. (1913): Johann Heinrich Kappelers Chronik von Frauenfeld. Nach dem im Bürgerarchiv Frauenfeld liegenden Manuskript (M. 15. S. 297-347). Thurgauische Beiträge zur vaterländischen Geschichte. Hsg. vom Historischen Verein des Kantons Thurgau. 53. Heft, S. 40-102.

- Schatzmann, R. (2006): Ein Erdbeben? - Die Koloniestadt Augusta Raurica als Fallbeispiel. Mittelalter, Moyen Age, Medioevo, Temp medieval. Zeitschrift des Schweizerischen Burgenvereins. 11. Jahrgang 2006/3, S. 165-170.

- Scheuchzer, J. (1706-1708): Beschreibung der Natur-Geschichten des Schweizerlands. Zürich.

- Scheuchzer, J. (1716): Helvetiae Stoicheiographia. Orographia et Oreographia. Oder Beschreibung der Elementen/Grenzen und Berge des Schweitzerlands, Zürich (1. Teil der Helvetiae Historia Naturalis Oder Natur-Historie des Schweizerlandes).

- Scheuchzer, J. (1746): Natur-Geschichte des Schweitzerlandes, samt seinen Reisen über die Schweizerische Gebürge, 2 Vol., ed. J. G. Sulzer, Zürich.

- Schiess, T. (1906): Bullingers Korrespondenz mit den Graubündnern III. Teil Oktober 1566-Juni 1575. S. 427-430.

- Schiess, T. (1911): Johannes Kesslers Sabbata. St. Galler Reformationschronik 1523-1539. Bearbeitung. Leipzig.

- Schilling, D. (1981): Die Schweizer Bilderchronik des Luzerners Diebold Schilling. Sonderausgabe des Kommentarbandes zum Faksimile der Handschrift S. 23 fol. in der Zentralbibliothek Luzern. Hsg. A. Schmid, Faksimile-Verlag Luzern.

- Schmedes, E., Loibl, R., Gebrande, H. (1992): Ein Schadensbeben in Regensburg am 8. Februar 1062 - eine Fehlinterpretation historischer Quellen. Geologisches Jahrbuch, Reihe E 1992.

- Schmieder, C. (1802): Die Geognosie nach chemischen Grundsätzen dargestellt. Leipzig.

- Schwarz-Zanetti, G. (1998): Grundzüge der Klima- und Umweltgeschichte des Hochund Spätmittelalters in Mitteleuropa. Diss. Univ. Zürich, Zürich (Kurzfassung des historischen Teils der Doppeldissertation 1992).

- Schwarz-Zanetti, G. (2008a): Das falsche Basler Erdbeben vom 12. Mai 1021 - ein humanistischer Irrtum. Nachbeben. Eine Geschichte der Erdbeben in der Schweiz, Bern, Gisler, Fäh, Giardini, Hsg., S. 25-31. 
- Schwarz-Zanetti, G. (2008b): Das Churwaldner Erdbeben vom 3. September 1295. Nachbeben. Eine Geschichte der Erdbeben in der Schweiz, Bern, Gisler, Fäh, Giardini, Hsg., S. 50, S. 35-40.

- Schwarz-Zanetti, G. (2008c): Schäden des Basler Bebens 1356 bis in den Odenwald oder ein Schadenbeben am 8. oder 9. Mai 1357? Unbeantwortete Fragen aus der Werkstatt der historischen Seismologie. Exkurs. Nachbeben. Eine Geschichte der Erdbeben in der Schweiz. Gisler, Fäh, Giardini, Hsg., S. 50.

- Schwarz-Zanetti, G. (2008d): Von dem Donnerstein gefallen im Jahr 1492 in Ensisheim. Exkurs. Nachbeben. Eine Geschichte der Erdbeben in der Schweiz. Gisler, Fäh, Giardini, Hsg., S. 51.

- Schwarz-Zanetti, G. (2008e): Erdbebenserie mit Flutwelle und Bergsturz vom 11.14. März 1584 im Genferseegebiet. Nachbeben. Eine Geschichte der Erdbeben in der Schweiz, Bern, Gisler, Fäh, Giardini, Hsg., S. 53-60.

- Schwarz-Zanetti, G. (2008f): Das Erdbeben in Unterwalden vom 16. September 1601 und die nachfolgende Flutwelle im Vierwaldstättersee. Nachbeben. Eine Geschichte der Erdbeben in der Schweiz, Bern. Gisler, Fäh, Giardini, Hsg., S. 61-68.

- Schwarz-Zanetti, G. (2008g): Das Wallis bis 1700: eine seismologische Terra incognita? Nachbeben. Eine Geschichte der Erdbeben in der Schweiz, Bern. Gisler, Fäh, Giardini, Hsg., S. 69-73.

- Schwarz-Zanetti, G. (2008h): Der Bergsturz von Plurs vom 4. September 1618. Exkurs. Nachbeben. Eine Geschichte der Erdbeben in der Schweiz, Bern, Gisler, Fäh, Giardini, Hsg., S. 74.

- Schwarz-Zanetti, G. \& W. (1992): Grundzüge der Klima- und Umweltgeschichte des Hoch- und Spätmittelalters in Mitteleuropa. Unpublizierte Doppel-Dissertation, Historisches Seminar und Geographisches Institut der Universität Zürich; Historisches Institut der Universität Bern. 647 S.

- Schwarz-Zanetti, G. \& W. (1992) Klimadatenbank EuroClimHist, Mittelalter Mitteleuropa 1, Historisches Institut der Universität Bern und Öschger Centre for Climate change Research, Bern. Datenbank zur Dissertation.

- Schwarz-Zanetti, G., Deichmann, N., Fäh, D., Masciadri, V., and Goll, J. (2004): The Earthquake in Churwalden (CH) of September 3, 1295. Eclogae Geol. Helv. - Swiss J. Geosciences, 97, S. 255-264.

- Schwarz-Zanetti, G., Deichmann, N., Fäh, D., Masciadri, V., Kästli, P., Schnellmann, M., Jimenez, M. (2006a): Das Unterwaldner Erdbeben vom 18. September 1601. Naturkatastrophen in der Zentralschweiz. Der Geschichtsfreund, 159, S. 11-28, 2006.

- Schwarz-Zanetti G., Fäh D., Schibler R., Masciadri V., Kästli P., and Giardini D. (2003): The earthquake in Unterwalden and the rockslide from the Bürgenstock into Lake Lucerne on September 18, 1601. Eclogae geol. Helv. - Swiss J. Geosciences, 96. 
- Schwarz-Zanetti, G., Fäh, D., Masciadri, V., Kästli, P., Gache S. (in prep., 2011): Two large earthquakes and a flood wave in Western Switzerland in the $16^{\text {th }}$ century: 1524 in Ardon, VS, and 1584 in Aigle, VS. Swiss journal of geosciences (in prep., working titel).

- Schwarz-Zanetti, G., Gisler, M., Fäh, D., Kästli, P. (2006b): Interdisziplinäre Rekonstruktion des Basler Erdbebens von 1356 an der ETHZ. Ein Werkstattbericht. Mittelalter - Moyen Age - Medioevo - Temp medieval, Zeitschrift des Schweizerischen Burgenvereins, S. 140-144.

- Schwarz-Zanetti, G., Masciadri, V., Fäh, D. \& Kästli, P. (2007): The false Basel earthquake of May 12, 1021. Journal of Seismology, 12, S. 125-129.

- Senn, N. (1860): Werdenberger Chronik: Ein Beitrag zur Geschichte der Kantone St. Gallen und Glarus. Chur.

- Sieberg, A. (1940): Beiträge zum Erdbebenkatalog Deutschlands und angrenzender Gebiete für die Jahre 58 bis 1799. Mitteilungen des Deutschen Reichs-Erdbebendienstes. Hsg. von der Reichsanstalt für Erdbebenforschung in Jena. Heft 2. Berlin. S. 3-111.

- SisFrance: Sismicité de la France. Histoire et caractéristiques des séismes ressentis en France. http//www.sisfrance.net.

- Sonnabend, H. (1999): Naturkatastrophen in der Antike. Wahrnehmung - Deutung Management. Stuttgart.

- Spon, J. (1680): Histoire de la ville et de l'estat de Genève. Depuis les premiers siècles de la fondation de la Ville jusqu' à Present: Tirée fidellement des manuscrits, par Jacob Spon Docteur Medecin aggregé au College de Lyon. Seconde Edition reveuë \& corrigé. Tome II. Amaulry, Lyon.

- Sprecher, von, A. (1992): Aus Bartholomäus Anhorns Chronik der Stadt Maienfeld. Selbstverlag des Verfassers.

- Steinemann, E. (1939): Stein am Rhein, Kirchenbücher 1559-1638. Pfarrer Hans Ulrich Grob und seine Einträge im ältesten Kirchenbuch von Stein-am-Rhein. Schaffhauser Beiträge zur Vaterländischen Geschichte.

- Stettler, M. (1626 und 1627): Schweitzer-Chronic. Das ist gründliche und wahrhaffte Beschreibung der ... Geschichten welche sich bey löblicher Eydgnosschafft seyt etlich hundert Jahren her verloffen ... . Bern.

- Stichler, C. (1906): Altzürcherische Erdbebenerinnerungen und Ähnliches. Zürcher Wochenchronik, Jg. 8, Nr. 22, Fortsetzung 23. Zürich.

- Stöckli, W. (2009): Brief vom 14. August 2009, Atelier d'archéologie médiévale, Moudon, an Gabriela Schwarz-Zanetti, SED. Im historischen Archiv des SED, Mecos 9650.

- Strasser, M. (2008): Quantifying Late Quaternary natural hazards in Swiss lakes: subaquatic landslides, slope stability assessments, paleoseismic reconstructions and lake outbursts. (Beiträge zur Geologie der Schweiz. Geotechnische Serie; Lfg. 95, Diss. ETH Zürich, Nr. 17285). 
- Stucchi, M. e Albini, P. (1988): Studi di sismica storica. In: ISMES, Studio di sismica storica e strumentale per l'Alta Valtellina, rapporto ASP-3946/RAT-URM-009, Bergamo, S. 1-194.

- Stumpf, J. (1548): Gemeiner loblicher Eydgnoschaft Stetten, landen und Völckeren Chronikwirdiger thaaten beschreybung [...], Froschauer, Zürich.

- Tauber, J. (1980): Herd und Ofen im Mittelalter. Untersuchungen zur Kulturgeschichte am archäologischen Material vornehmlich der Nordwestschweiz (9.-14. Jahrhundert). Schweizer Beiträge zur Kulturgeschichte und Archäologie des Mittelalters, Band 7. Hsg. vom Schweizerischen Burgenverein.

- Tribolet, de, M. (1876): Liste des tremblements de terre à Neuchâtel, de l'an 1313 à 1875. Bulletin de la Société des Sciences Naturelles de Neuchâtel, tôme X, S. 358372. Neuchâtel.

- Thorndike, L. (1949): A daily weather record from the years 1399 to 1406 A.D. ISIS, International Review devoted to the History of Science and Civilization, Vol. 32,2: S. 304-323.

- Thorndike, L. (1966): A daily weather record from the years 1399 to 1401. ISIS, An International Review devoted to the History of Science and its cultural influences, Vol. 57: S. 90-101. (Zusammenfassende englische Transkription der ersten Jahre).

- Tribolet, de, M. (1876): Tremblements de terre ressentis dans le canton de Neuchâtel. Bulletin de la société des sciences naturelles de Neuchâtel. 1874 à 1876. Tome dixième. S. 358-372.

- Trümpi, C. (1774): Neuere Glarner-Chronick: begreift in sich: ... samt einem Anhang/ zusammen getragen von Christoph Trümpi, Diener des Worts Gottes, an der Kirch zu Schwanden. Steiner, Winterthur.

- Tschamser, M. (1864): Annales oder Jahrs-Geschichten der Baarfüsern oder Minderen-Brüdern S. Franc. Ord. insgemein Conventualen genannt, zu Thann. Colmar.

- Tschudi, J. H. (1714): Beschreibung Des Lobl. Orths und Lands Glarus. Vorstellende So wol diss Lands natürliche Beschaffenheit und Regiments Politische Verfassung/als auch und fürnemlich die Geschichten/die sich in und mit demselbigen zugetragen. Auss allerhand glaubwürdigen Documentis zusammen gebracht/und nach der Jahr-Ordnung biss auf gegenwertige Zeit aussgeführt. Samt einem Anhang von dem Toggenburger-Geschäfft. Zürich.

- Tschudi, N. (1869): Die Bergstürze am vordern Glärnisch bei Glarus in den Jahren 1593 und 1594. Jahrbuch des historischen Vereins des Kantons Glarus. Fünftes Heft, S. 89-100.

- Vadian (von Watt) J. (1879): Fragment einer römischen Kaisergeschichte. Geschichte der Fränkischen Könige. Epitome.-Diarium. Ed. Ernst Götzinger. St. Gallen.

- Vischer, W., Boss, H. Hrsg. (1880): Johannis Knebel Capellani Ecclesiae Basiliensis Diarium. Hans Knebels des Kaplans am Münster zu Basel Tagebuch, Sept. 1473Juni 1476. Basler Chroniken, Bd. 2. 
- Vischer, W. \& Stern, A. Hsg. (1872): Die Chronik des Fridolin Ryff 1514-1541, mit der Fortsetzung des Peter Ryff 1543-1585. Basler Chroniken, Band 1. Leipzig.

- Vitoduranus, J. [Johannes von Winterthur] (1924): Chronica Iohannis Vitodurani. MGH SRG III,. ed. F. Baethgen, Berlin.

- Vogt, J. (1987): L'activité sismique en 1650 aux confins de la Suisse, de la France et de l'Allemagne. Bulletin historique de la ville de Mulhouse, t. 3, S. 343-349.

- Vogt, J. (1992): Ouragan et séisme (France de l'Est, Allemagne et Suisse, 1523 et 1756) Revue Géographique de l'est. S. $3 f$.

- Volger, G. (1857): Untersuchungen über das Phänomen der Erdbeben in der Schweiz: seine Geschichte, ... . Theil 1: Chronik der Erdbeben in der Schweiz. Bd. 1, Gotha.

- Wackernagel, W. (1856): Das Erdbeben von 1356 in den Nachrichten der Zeit und der Folgezeit bis auf Christian Wurstisen. Basel im vierzehnten Jahrhundert. Geschichtliche Darstellungen zur fünften Säcularfeier des Erdbebens am S. Lucastage 1356. Hsg. von der Basler Historischen Gesellschaft. S. 211-250.

- Walch, B. (1950): La chronique de Lucelle. Chronicon R. P. Bernardini Walch. Senioris et archivarii monasterii Lucellensis, de abbatibus Lucellensibus et rebus memorabilibus sub iis gestis usque 1445. Publié d'après le manuscript de Faverois, maintenant à la Bibliothèque de la Société belfortaine d'émulation par Louis Stouff. Strasbourg. Collection d'études sur l'histoire du droit et des institutions de l'Alsace.

- Walser, G. (1740): Neue Appenzeller-Chronick oder Beschreibung Des Cantons Appenzell Der Innern- und Aussern -Rooden (...), St. Gallen.

- Wattenbach, W., Holtzmann, R., (1967-1971): Deutschlands Geschichtsquellen im Mittelalter: die Zeit der Sachsen und Salier. Neuausgabe von F.-J. Schmale. Darmstadt: Böhlau.

- Wechsler, E. (1987): Das Erdbeben von Basel 1356. Teil 1: Historische und kunsthistorische Aspekte. Publikationsreihe des Schweizerischen Erdbebendienstes ETHZürich, Nr. 102.

- Weiss, J. (1914): Elementarereignisse im Gebiete Deutschlands. Systematische Sammlung der Nachrichten über Elementarereignisse und physisch-geographische Verhältnisse. Hsg. von dem Gesamtverein der deutschen Geschichts- und Altertumsvereine. I. Die Elementarereignisse vom Beginn unserer Zeitrechnung bis zum Jahre 900. Wien, Holzhausen.

- Weller, E. (1862-64): Annalen der poetischen National-Literatur der Deutschen im 16. und 17. Jahrhundert. Nach den Quellen bearb. von Emil Weller. Freiburg i. Br.: Herder.

- Wild, W. (2006): „Unter schrecklichem Knallen barsten die Mauern“ - Auf der Suche nach archäologischen Spuren von Erdbebenkatastrophen. Mittelalter, Moyen Age, Medioevo, Temp medieval. Zeitschrift des Schweizerischen Burgenvereins. 11. Jahrgang 2006/3. S. 145-164.

- Wolf, P. \& H. (1989): Das Erdbeben in Regensburg von 1062 - Wirklichkeit oder wissenschaftliches Phantom. Die Oberpfalz 77, S. 35-43. 
- Wolf, R. (1856/1857): Guggenbühl, Wyn Rechnung der Statt Zürich. Von ano 1421 biss auff disse gegenwärtige Zeit. Vierteljahresschrift der Naturforschenden Gesellschaft in Zürich, 1856-57.

- Wolfart, K., Hsg. (1909/1979): Geschichte der Stadt Lindau im Bodensee. Bd. 2, Lindau.

- Wolfart, K. (1912): Lindauer Erdbeben-Chronik. Neujahrsblätter des Museumsvereins Lindau i. B. Nr. 2. S. $29 f$.

- Wolff, S. (2008): Die „Konstanzer Chronik“ Gebhart Dachers. „By des Byschoffs zyten volgiengen disz nachgeschriben ding vnd sachen ..... Codex Sangallensis 646. Edition und Kommentar. Konstanzer Geschichts- und Rechtsquellen, Bd. 40. Hsg. vom Stadtarchiv Konstanz.

- Wurstisen, C. (1580): Bassler Chronick/Darinn alles/was sich in Oberen Teutschen Landen/nicht nur in der Statt und Bistumbe Basel/von jhrem ursprung her/nach Ordnung der Zeiten/in Kirchen und Welt händlen/biss in das gegenwirtige M.D. LXXX Jar/gedenckwirdigs zuogetragen: ... . Henricpetri, Basel.

- Zehnder, L. (1976): Volkskundliches in der älteren schweizerischen Chronistik. Schriften der Schweizerischen Gesellschaft für Volkskunde, Bd. 60. Basel.

- Zeitung, Zwo warhafftige newe. Die erste, von dem erschrecklichen Erdbide, so den 8. September durch gantz Teutschland ergangen. Die ander, von der Stadt Willissaw im Schweizerland, wie alda Fewr vom Himmel gefallen .... geschehen dieses 1601. Jahres. Hans Schrötter, Basel.

- Zeller, J. (1913): Das Erdbeben vom 3. Januar 1117. Ein Beitrag zur archäologischen Erforschung Rottenburgs. Württembergische Vierteljahrshefte für Landesgeschichte. Neue Folge. XXII. Jahrgang. 1913. S. 255-271.

- Ziegler, J. (1674): Grundlicher Bericht/Von den natürlichen ursachen Der Erdbidmen. Samt angehenkter Historischer Erzehlung/was mehrentheils darauf in unserem geliebten Vatterland erfolget. Von Jacob Ziegler/der Artzney Doctoren zu Zürich. Schaufelberger, Zürich.

- Zwinger, T. (1650): Christliche Wahrnungs- und Buoss-Predigt/Von dem Erdbidem/alss einem grossen gewaltigen Werck und Zornzeichen Gottes des Allerhöchsten. (...) den 11. September (...), Genath, Basel.

\subsection{Weiterführende Literatur}

- Alexandre, P. (1984): Problèmes de méthode relatifs a l'étude des séismes médiévaux. IVèmes rencontres internationales d'archéologie et d'histoire d'Antibes. Tremblements de terre Histoire et archéologie, 2./3./4. Novembre 1983, ed. Association pour la promotion et la diffusion des connaissances archéologiques, Valbonne: S. 221-226. 
- Ambraseys, N.N. (1971): Value of Historical Records of Earthquakes. Nature 232: S. 375-379.

- Boschi, E. (2000). A „new generation' earthquake catalogue“. In Catalogue of Strong Italian Earthquakes from 461 B.C. to 1997. Introductory Texts and CD-ROM. Annali di Geofisica 43, 4: S. 609-620.

- Fäh, D., et al. (2003): Earthquake Catalog of Switzerland (ECOS) and the Related Database. Eclogae Geol. Helv. Swiss Journal of Geosciences 96, S. 219-236.

- Gisler, M. (2003): Historical Seismology in Switzerland: Reflections on Issues and Insights. Environment and History 9/2, S. 215-237.

- Gisler, M. (2007): Göttliche Natur? Formationen im Erdbebendiskurs der Schweiz des 18. Jahrhunderts, Zürich.

- Grünthal, G. ed. (1998): European Macroseismic Scale 1998 (EMS-98), Cahiers du Centre Européen de Géodynamique et de Séismologie 15, Helfent-Bertrange (Luxembourg).

- Guidoboni, E. (2000): Method of investigation, typology and taxonomy of the basic data: navigating between seismic effects and historical contexts. Catalogue of Strong Italian Earthquakes from 461 B.C. to 1997. Introductory Texts and CD-ROM. Annali di Geofisica 43, 4: S. 621-666.

- Musson, R. M. W. (1998): Intensity assignments from historical earthquake data: issues of certainty and quality. Annali di Geofisica 41, 1: S. 79-91.

- Schweizerische Erdbebenkommission/Schweizerischer Erdbebendienst (18811962; 1972-1974): Jahresberichte des Schweizerischen Erdbebendienstes, Zürich.

- Stucchi, M. (1993): Through catalogues and historical records: an introduction to the project „Review of Historical Seismicity in Europe“. Historical Investigation of European Earthquakes. Materials of the CEC Project „Review of Historical Seismicity in Europe“ 1, ed. M. Stucchi, Milano: S. 3-14.

- Stucchi, M., Bellani, A. and Chignola, E. (1991): Behind the intensity map: assessing the quality of historical-seismological information. In Proceedings of the Third International Symposium on Historical Earthquakes in Europe, ed. J. Kozák, Prague: S. 41-57.

- Vogt, J. (1979): Problèmes de Méthode. Les tremblements de terre en France, ed. B. Cadiot et al. (publié sous la direction de Jean Vogt), Orléans: S. 9-15.

- Vogt, J. (1993): Historical seismology. Some notes on sources for seismologists. Historical Investigation of European Earthquakes. Materials of the CEC Project „Review of Historical Seismicity in Europe“ 1, ed. M. Stucchi, Milano: S. 15-24. 


\section{Anhang: Die zwölfstufige „Europäische Makroseismische Skala 1998“" (EMS-98)}

Die zwölfstufige „Europäische Makroseismische Skala 1998“ (EMS-98) ist die heute in Europa gültige - wenn auch nicht die einzig angewandte - Intensitätsskala. Sie beschreibt die während eines Erdbebens beobachteten Phänomene beziehungsweise Schadensbilder. Alle Intensitätsangaben in dieser Publikation beziehen sich auf diese Skala. Die folgende Kurzform stellt eine sehr starke Vereinfachung und Generalisierung der ausführlichen Fassung (Grünthal, 1998) dar.

\begin{tabular}{|c|c|c|}
\hline EMS-98 & Intensität & Beschreibung der maximalen Wirkung \\
\hline I (1) & Nicht verspürt & Nicht zu verspüren. \\
\hline II (2) & Kaum verspürt & $\begin{array}{l}\text { Erschütterungen werden nur in einzelnen Fällen } \\
\text { von Personen in völliger Ruhe in Gebäuden wahr- } \\
\text { genommen. }\end{array}$ \\
\hline III (3) & Schwach (verspürt) & $\begin{array}{l}\text { Das Erdbeben wird in Gebäuden von wenigen Per- } \\
\text { sonen verspürt. Personen in Ruhe fühlen ein } \\
\text { Schwanken oder leichtes Zittern; hängende Ge- } \\
\text { genstände schwingen leicht; es entstehen keine } \\
\text { Schäden. }\end{array}$ \\
\hline IV (4) & $\begin{array}{l}\text { Grösstenteils } \\
\text { beobachtet }\end{array}$ & $\begin{array}{l}\text { Das Beben wird in Gebäuden von vielen Personen } \\
\text { und im Freien von wenigen verspürt; wenige Per- } \\
\text { sonen werden wach, die Stärke der Erschütterun- } \\
\text { gen ist nicht erschreckend. Die Vibrationen sind } \\
\text { mässig; ein leichtes Erzittern von Gebäuden, Bet- } \\
\text { ten, Stühlen usw. kann verspürt werden. Geschirr, } \\
\text { Gläser, Fenster und Türen klappern. Hängende } \\
\text { Objekte schwingen. In wenigen Fällen wird leich- } \\
\text { tes Mobiliar sichtbar gerüttelt und Holzwände } \\
\text { knarren. Es entstehen keine Schäden. }\end{array}$ \\
\hline V (5) & Stark & $\begin{array}{l}\text { Das Beben wird in Gebäuden von den meisten } \\
\text { Personen, im Freien von wenigen verspürt. Wenige } \\
\text { Personen erschrecken und rennen ins Freie. } \\
\text { Viele schlafende Personen erwachen. Ein starkes } \\
\text { Schütteln oder Rucken des ganzen Gebäudes, } \\
\text { des Zimmers oder der Möbel wird verspürt. Hän- } \\
\text { gende Objekte schwingen beträchtlich. Geschirr } \\
\text { und Gläser klirren aneinander. Kleine, kopflastige }\end{array}$ \\
\hline
\end{tabular}




\begin{tabular}{|l|l|l|}
\hline EMS-98 & Intensität & Beschreibung der maximalen Wirkung \\
& und/oder unsicher abgestützte Gegenstände kön- \\
nen verschoben werden oder fallen herunter. Tü- \\
ren und Fenster schlagen auf oder zu. In wenigen \\
Fällen zerbrechen Fensterscheiben. Flüssigkeiten \\
schwingen auf und ab und können aus gut gefüll- \\
ten Behältern überschwappen. Leichte Schäden \\
an wenigen Gebäuden in eher schlechtem Zu- \\
stand sind möglich.
\end{tabular}




\begin{tabular}{|l|l|l|}
\hline EMS-98 & Intensität & Beschreibung der maximalen Wirkung \\
\hline $\begin{array}{l}\text { werden gedreht. Wellen sind auf weichem Unter- } \\
\text { grund sichtbar. Sogar gut gebaute gewöhnliche } \\
\text { Bauten zeigen sehr schwere Schäden. Viele } \\
\text { schwächere Bauten stürzen ein, teilweise Einsturz } \\
\text { tragender Bauteile. }\end{array}$ \\
\hline X (10) & Sehr zerstörend & $\begin{array}{l}\text { Viele gut gebaute Häuser werden zerstört oder } \\
\text { erleiden schwere Beschädigungen. }\end{array}$ \\
\hline XI (11) & Verwüstend & $\begin{array}{l}\text { Die meisten Bauwerke, selbst einige mit gutem } \\
\text { erdbebengerechtem Konstruktionsentwurf und } \\
\text { guter Konstruktionsausführung, werden zerstört. }\end{array}$ \\
\hline XII (12) & Vollständig verwüstend & \begin{tabular}{l} 
Nahezu alle Konstruktionen werden zerstört. \\
\hline
\end{tabular}
\end{tabular}


AG an der

AG an der ETH Zurich

(c) vdf $t$

wich (o) vif Hochschulverlag AG an der ETHIZUric

Aurich (c) voff Hochschulverlag

an ETHZürich () valf Hochso

- wherlag Af an der ETHZ Zurich (O) vaf Hoch:

an der ETHZ Zurich (c) vif Ho

AG an der ETHZ Zurich (9) vaf Hochschulve

Chulverlag AG an der ETHZürich (c) vdf

1

zuirich

vof Hochschulverlag 
Als Ergänzung zur Online-Version des Schweizer Erdbebenkatalogs ECOS-09 (http://www.seismo.ethz.ch) steht mit diesen zweibändigen GRUNDLAGEN DES MAKROSEISMISCHEN ERDBEBENKATALOGS DER SCHWEIZ erstmals eine historischkritische Diskussion der wichtigsten nichtinstrumentellen Schweizer Erdbebendaten zur Verfügung. Aus dem Zeitraum 1000-1878 werden alle bekannten Schweizer Erdbeben ab Intensität 6, zahlreiche Falschmeldungen und einige interessante schwache Beben ausführlich kommentiert.

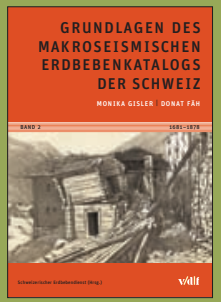

BAND 2

Monika Gisler, Donat Fäh

Grundlagen des Makroseismischen

Erdbebenkatalogs der Schweiz 1681-1878

Schweizerischer Erdbebendienst (Hrsg.)

ISBN 978-3-7281-3237-6 